\title{
HOLOMORPHIC FUNCTIONS OF EXPONENTIAL TYPE AND DUALITY FOR STEIN GROUPS WITH ALGEBRAIC CONNECTED COMPONENT OF IDENTITY
}

S. S. Akbarov

October 29, 2018 


\section{Introduction}

Since in 1930s L. S. Pontryagin published his famous duality theorem for Abelian locally compact groups 27, the following problem engages the imagination of specialists in harmonic analysis from time to time: is it possible to generalize Pontryagin duality to non-Abelian locally compact groups in such a way that the dual object has the same nature as the initial one?

As is known, the first attempts to generalize Pontryagin duality did not meet this requirement: in the M. G. Krein theory, for instance, the dual object $\widehat{G}$ for a group $G$ is a block-algebra [20] (but not a group, unlike Pontryagin theory). Apparently, a deep peculiarity in human psychology manifests itself here, but such a harmless trait like asymmetry between $G$ and $\widehat{G}$ in the theory of representations - a trait that can be compared with difference between the left and the right in anatomy - leads to numerous and, because of the changing with time understanding of what the notion of group should mean, continuing attempts to build a duality theory, where, on the one hand, "all" groups are covered, and, on the other, the Pontryagin symmetry between initial objects and their duals is preserved.

In the category theory language, the unique one fitting for such speculative aspirations, one can formulate this task correctly using the following two definitions.

1. Let us call a contravariant functor $A \mapsto A^{*}: \mathfrak{K} \rightarrow \mathfrak{K}$ on a given category $\mathfrak{K}$ a duality functor in $\mathfrak{K}$, if its square, i.e. the covariant functor $A \mapsto\left(A^{*}\right)^{*}: \mathfrak{K} \rightarrow \mathfrak{K}$, is isomorphic to the identity functor $\operatorname{id}_{\mathfrak{K}}: \mathfrak{K} \rightarrow \mathfrak{K}$.

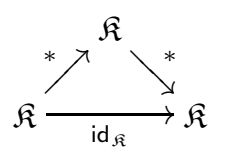

The passage to the dual group $G \mapsto G^{\bullet}$ in Pontryagin theory is an example of duality functor: the natural isomorphism between $G^{\bullet \bullet}$ and $G$ here is the mapping

$$
i_{G}: G \rightarrow G^{\bullet \bullet} \mid i_{G}(x)(\chi)=\chi(x), \quad x \in G, \chi \in G^{\bullet}
$$

On the contrary, say, in the category of Banach spaces the passage to the dual Banach space $X \mapsto X^{*}$ is not a duality functor (because there are non-reflexive Banach spaces).

2. Suppose we have:

(a) three categories $\mathfrak{K}, \mathfrak{L}, \mathfrak{M}$ with two full and faithful covariant functors $A: \mathfrak{K} \rightarrow \mathfrak{L}$ and $B: \mathfrak{L} \rightarrow \mathfrak{M}$ defining a chain of embeddings:

$$
\mathfrak{K} \subset \mathfrak{L} \subset \mathfrak{M},
$$

(b) two duality functors $K \mapsto K^{\bullet}: \mathfrak{K} \rightarrow \mathfrak{K}$ and $M \mapsto M^{*}: \mathfrak{M} \rightarrow \mathfrak{M}$ such that the functors $K \mapsto B\left(A\left(K^{\bullet}\right)\right)$ and $K \mapsto(B(A(K)))^{*}$ are isomorphic:

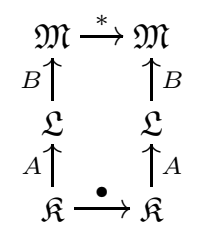

We shall call this construction a generalization of the duality $\bullet$ from the category $\mathfrak{K}$ to the category $\mathfrak{L}$.

On adding these terms into our armoury, we can formulate the task we are discussing as follows: are there any generalizations of Pontryagin duality from the category of Abelian locally compact groups to the 
category of arbitrary locally compact groups, and if yes, then which namely? The category diagram (B) in this formulation becomes as follows:

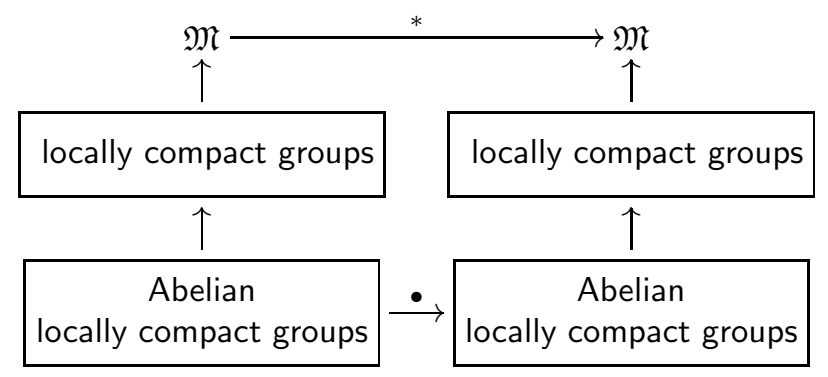

- and the key example here is the duality theory for finite groups, which can be regarded as a generalization of Pontryagin duality from the category of Abelian finite groups to the category of all finite groups:

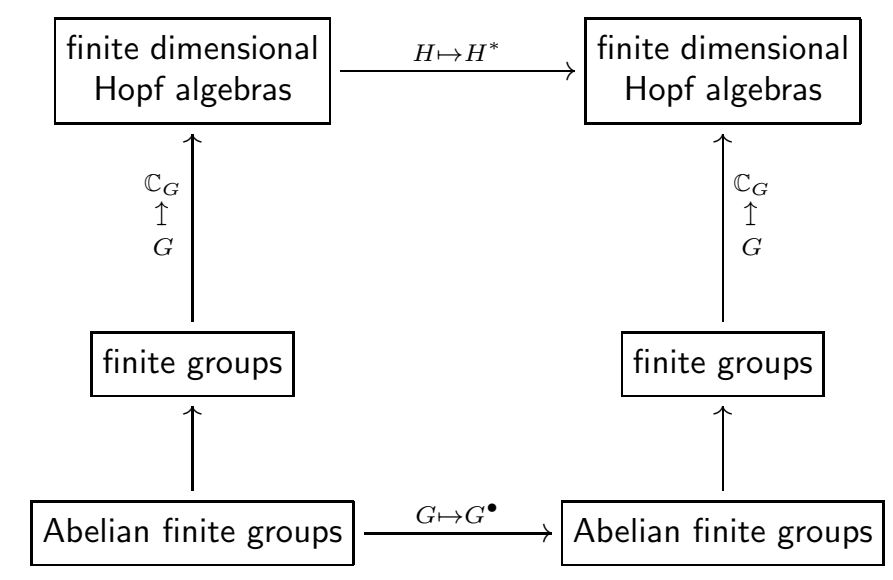

(here $G \mapsto \mathbb{C}_{G}$ is the passage to group algebra, and $H \mapsto H^{*}$ the passage to dual Hopf algebra). This example, apart from everything else, illustrates another guiding idea of the "general duality theory": if we want to reduce the representations of groups to representations of algebras, and therefore claim that the category $\mathfrak{M}$ consists of associative algebras, then these algebras $H$ must have some supplementary structure, which allows us to endow dual objects $H^{*}$ with a natural structure of associative algebra. Natural objects of that kind in general algebra are Hopf algebras. As a corollary, the constructions in "duality theory" usually resemble Hopf algebras, although as a rule differ from them, except for trivial cases when, for instance, the algebra has finite dimension.

A generalization of Pontryagin duality as it is presented in diagram (C) was suggested in 1973 independently by L. I. Vainerman and G. I. Kac from one side (43], 44], 45]), and by M. Enock and J.-M. Schwartz from another (11], 12, [13). The theory of Kac algebras they developed summarized a series of attempts made by different mathematicians, history of which, as well as the theory of Kac algebras itself, one can learn from monograph [10] by Enock and Schwartz. After 1973 the work in this direction did not cease, because on the one hand, some improvements were added into the theory (again see details in [10), and on the other, after the discovery of quantum groups in 1980s, mathematicians began to generalize the Pontryagin duality to this class as well. Moreover, the latter work is not finished by now - the theory of locally compact quantum groups which appeared on this wave is being actively developed by J. Kustermans, W. Pusz, P. M. Soltan, S. Vaes, L. Vainerman, A. Van Daele, S. L. Woronowicz (an impression of this topic one can find in collective monograph [28]). The idea of multiplier Hopf algebra suggested by A. Van Daele in 1990-s [41, 42], seems to be strategic in these investigations.

Despite the active work and impressive enthusiasm demonstrated by mathematicians engaged in this theme, the theories they suggest have a serious shortcoming: all the enveloping categories $\mathfrak{M}$ in these theories consist of objects, which are formally not Hopf algebras. The Kac algebras, for instance, although 
being chosen as a subclass among the objects called Hopf-von Neumann algebras, in fact are not Hopf algebras, because from the point of view of category theory in the definition of Hopf-von Neumann algebras, unlike the pure algebraic situation, two different tensor products are used simultaneously the projective tensor product for multiplication, and the tensor product of von Neumann algebras for comultiplication. In the theory of locally compact quantum groups the situation on this point seems to give even less hopes, because here the claim that the comultiplication must indispensably act into tensor product is rejected: in accordance with the above mentioned Van Daele's idea of multiplier Hopf algebras the comultiplication here is defined as an operator from $A$ into the algebra $M(A \otimes A)$ of multipliers on tensor product $A \otimes A$ (which is chosen here as minimal tensor product of $C^{*}$-algebras, see [21, 28).

In this paper we suggest another approach to the generalization of Pontryagin duality, where this unpleasant effect does not occur: in our theory the enveloping category $\mathfrak{M}$ consists of "true" Hopf algebras, of course in the categorical sense, i.e. the Hopf algebras defined in the same way as the usual Hopf algebras, but after replacing the category of vector spaces by a given symmetrical (in the more general case - braided) monoidal category (such Hopf algebras are sometimes called Hopf monoids, see [29, 35, 36, 49]).

The mission we set to ourselves is not quite a generalization of Pontryagin duality to the class of locally compact groups, as it is presented in diagram (C), but a solution of the same problem in the class of complex Lie groups. The fact is that among the four main branches of mathematics, where the idea of invariant integration manifests itself -

- general topology (where the groups with invariant integral are exactly locally compact groups),

- differential geometry (where this role belongs to Lie groups),

- complex analysis (where the reductive complex groups can be regarded as the groups with integral), and

- algebraic geometry (again with reductive complex groups),

- at least in the three first disciplines the generalization of Pontryagin duality has sense. In topology this problem takes form of diagram (C), in differential geometry one can consider the problem of generalization of Pontryagin duality from Abelian compactly generated Lie groups, say, to all compactly generated Lie groups, and in the complex analysis one can consider the problem of generalization of Pontryagin duality from the class of Abelian compactly generated Stein groups to the class of reductive complex groups (following 46, by a reductive group we mean complexification of a compact real Lie group).

We give a solution for the third of these problems. We begin our considerations with the algebra $\mathcal{O}(G)$ of holomorphic functions on a complex Lie group $G$, since this is a natural functional algebra in complex analysis (in the other three disciplines this role belongs to the algebra $\mathcal{C}(G)$ of continuous functions, the algebra $\mathcal{E}(G)$ of smooth functions, and the algebra $\mathcal{R}(G)$ of polynomials). The idea we suggest as a heuristic hypothesis in this paper - and its justification we see in our work - is as follows. It seems likely, that to each of the three first disciplines in our list - general topology, differential geometry and complex analysis - from the point of view of the topological algebras used in these disciplines, corresponds some class of seminorms, intrinsically connected to this class of algebras. What those seminorms should be in general topology and in differential geometry we cannot say right now, but the class of submultiplicative seminorms, i.e. seminorms defined by the inequality

$$
p(x \cdot y) \leqslant p(x) \cdot p(y)
$$

is intrinsically connected with complex analysis (this idea is inspired to us by A. Yu. Pirkovskii's results on the Arens-Michael envelopes of topological algebras - see [26]).

To give exact meaning to the words "intrinsically connected", we need to introduce the following two functors and one category of Hopf algebras:

1) The Arens-Michael envelope $A \mapsto A^{\odot}$. The algebras whose topology is generated by submultiplicative seminorms and satisfies supplementary condition of completeness, are called Arens-Michael 
algebras (we discuss them in $\$ 5$ ). Each topological algebra $A$ has a nearest "from outside" ArensMichael algebra - we denote it by $A^{\odot}$ - called Arens-Michael envelope of $A$. This algebra is a completion of $A$ with respect to the system of all continuous submultiplicative seminorms on it.

2) Passage to dual stereotype Hopf algebra $H \mapsto H^{\star}$. In the work [1] we discussed in detail the symmetrical monoidal categories $(\mathfrak{S t e}, \odot)$ and $(\mathfrak{S t e}, \circledast)$ of stereotype spaces (with injective $\odot$ and projective $\circledast$ tensor products). The Hopf algebras in these categories are called respectively injective and projective stereotype Hopf algebras. The passage to the dual stereotype space $H \mapsto H^{\star}$ establishes an antiequivalence between these categories of Hopf algebras.

3) Category of holomorphically reflexive Hopf algebras. The functors $\varnothing$ and $\star$ allow us to consider the category of projective and at the same time injective stereotype Hopf algebras $H$, for which the successive application of the operations $\varnothing$ and $\star$ always leads to injective and at the same time projective Hopf algebras and, if we begin with $\varnothing$, then at the fourth step this chain leads back to the initial Hopf algebra (of course up to an isomorphism). We depict this closed chain by the following reflexivity diagram,

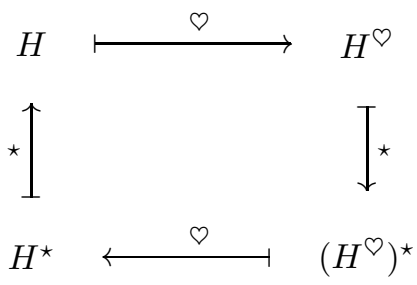

and call such Hopf algebras $H$ holomorphically reflexive (see accurate definition in $\S 5(\mathrm{a})$ ). The duality functor in the category of such Hopf algebras is, certainly, the operation $H \mapsto\left(H^{\vee}\right)^{\star}$.

The main result of our work, which clarifies our hints about "seminorms, intrinsically connected with complex analysis", is that the algebra $\mathcal{O}^{\star}(G)$ of analytical functionals on a compactly generated Stein group $G$ with algebraic connected component of identity is a holomorphically reflexive Hopf algebra. To prove this we introduce in this paper the algebra $\mathcal{O}_{\exp }(G)$ of holomorphic functions of exponential type on group $G$, which is a subalgebra in the algebra $\mathcal{O}(G)$ of all holomorphic functions on $G$. The reflexivity diagram (E) for $\mathcal{O}^{\star}(G)$ is as follows:

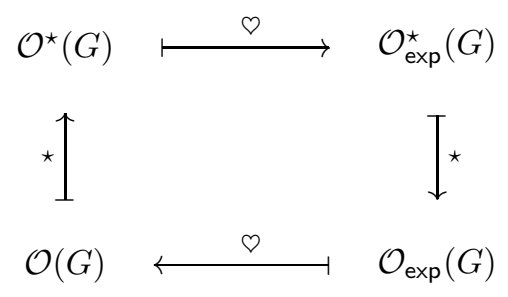

For the case of Abelian groups the operation $\odot$ becomes naturally isomorphic to the usual Fourier 
transform, so this diagram takes the form:

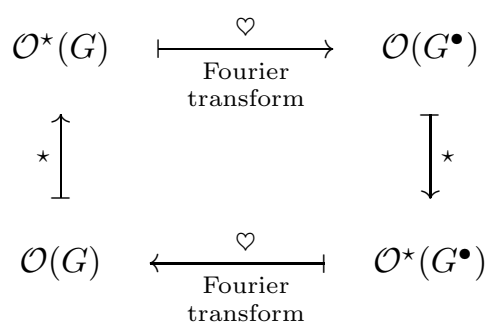

(the dual group $G^{\bullet}$ for an Abelian compactly generated Stein group $G$ is defined as the group of all homomorphisms from $G$ into the multiplicative group $\mathbb{C}^{\times}:=\mathbb{C} \backslash\{0\}$ of non-zero complex numbers). This, in particular, implies the isomorphism of functors

$$
\mathcal{O}^{\star}\left(G^{\bullet}\right) \cong\left(\left(\mathcal{O}^{\star}(G)\right)^{\mathcal{D}}\right)^{\star},
$$

which gives a generalization of Pontryagin duality in the complex case, and the diagram (B) here takes the form:

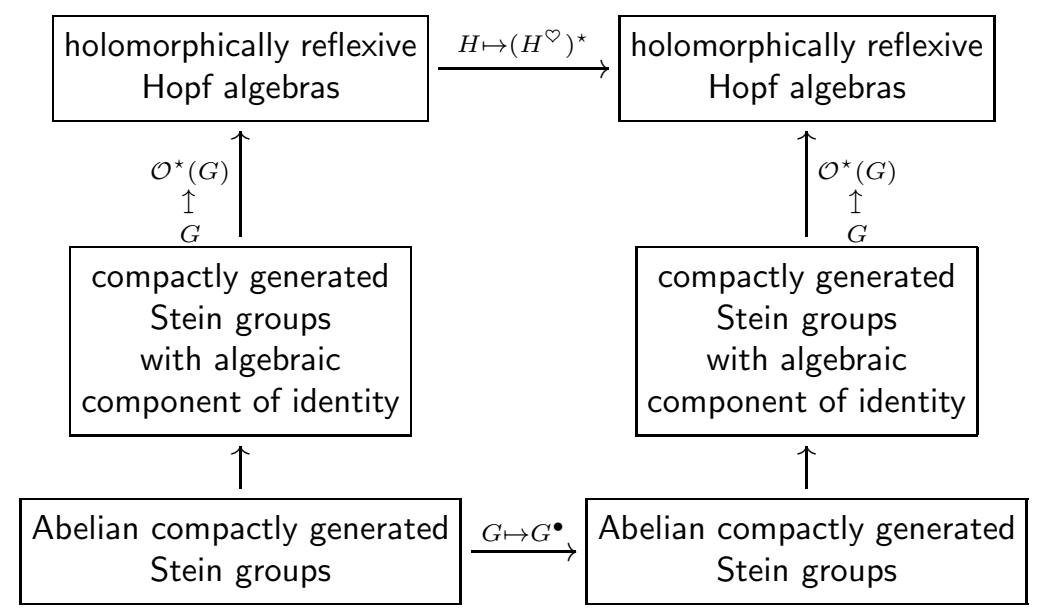

Since every reductive group is algebraic, this indeed will be a solution of the third problem in our list.

We show in addition that the holomorphic duality we introduce here does not limit itself to the class of compactly generated Stein groups with algebraic connected component of identity, but extends to quantum groups. As an example we consider the quantum group ' $a z+b$ ' of quantum affine automorphisms of complex plane (see [48, 40, 47, 28]). We prove that ' $a z+b$ ' is a holomorphically reflexive Hopf algebra in the sense of our definition.

The author thanks sincerely D. N. Akhieser, O. Yu. Aristov, P. Gaucher, A. Ya. Helemskii, A. Huckleberry, E. B. Katsov, Yu. N. Kuznetsova, T. Maszczyk, S. Yu. Nemirovskii, A. Yu. Pirkovskii, V. L. Popov, P. Soltan, A. Van Daele for innumerous consultations and help during the work on this paper. Besides that the idea of proof of Propositions 4.1, 7.12 and Lemma 7.9 belongs to Yu. N. Kuznetsova.

\section{$\S 0 \quad$ Stereotype spaces}

Stereotype spaces we are speaking about in this section were studied by the author in detail in [1] (see also [2, 3]). 


\section{(a) Definition and typical examples}

Let $X$ be a locally convex space over $\mathbb{C}$. Denote by $X^{\star}$ the space of all linear continuous functionals $f: X \rightarrow \mathbb{C}$, endowed with the topology of uniform convergence on totally bounded sets in $X$. The space $X$ is called stereotype, if the natural mapping

$$
\mathrm{i}_{X}: X \rightarrow\left(X^{\star}\right)^{\star} \quad \mid \quad \mathrm{i}_{X}(x)(f)=f(x), \quad x \in X, f \in X^{\star}
$$

is an isomorphism of locally convex spaces. Clearly the following theorem holds:

Theorem 0.1. If $X$ is a stereotype space, then $X^{\star}$ is also a stereotype space.

It turns out that stereotype spaces form a very wide class, what can be illustrated by the following diagram:

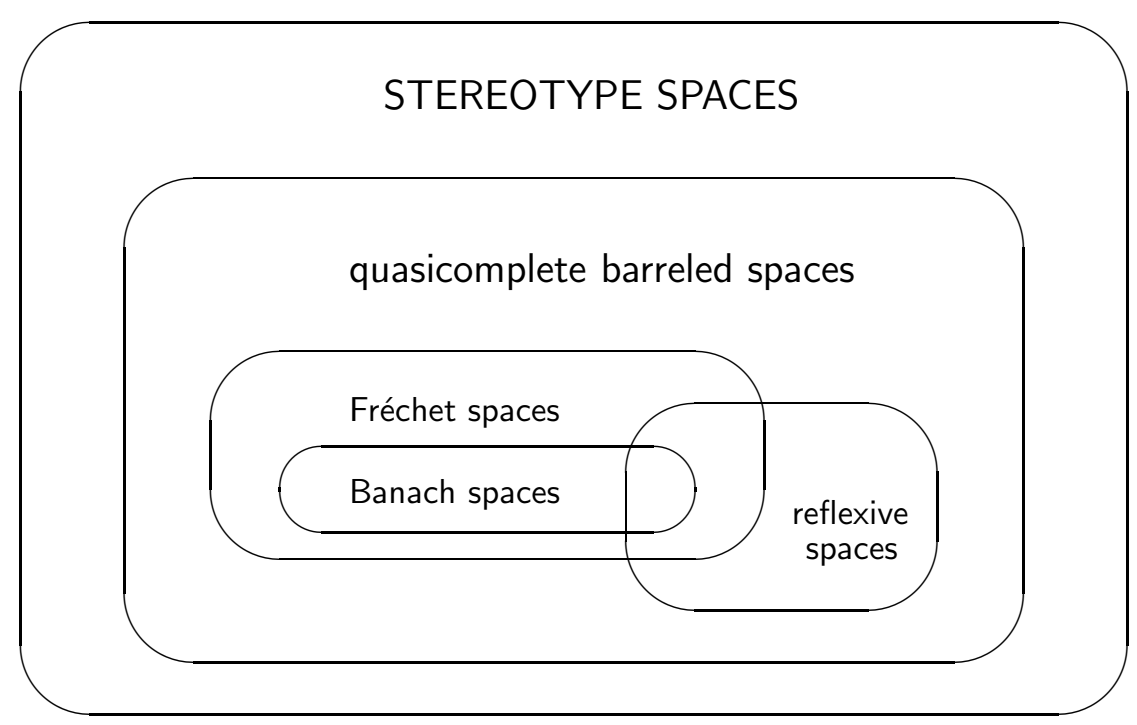

Fréchet spaces and Banach spaces will be of special interest for us in this picture, so we shall consider them in detail.

Example 0.1 (Fréchet spaces and Brauner spaces). Every Fréchet space $X$ is stereotype 7]. Its dual space $Y=X^{\star}$ is also stereotype by theorem 0.1. If $\left\{U_{n}\right\}$ is a countable local base in $X$, then the polars $K_{n}=U_{n}^{\circ}$ form a countable fundamental system of compact sets in $Y$ : every compact set $T \subseteq Y$ is contained in some compact set $K_{n}$ (this means by the way that $Y$ cannot be Fréchet space, if $X$ infinite dimensional). The spaces $Y$ dual to Fréchet spaces $X$ (in the sense of our definition) were originally considered by K. Brauner in [7], and we call them Brauner spaces. Their characteristic properties are listed in the following proposition.

Proposition 0.1. For a locally convex space $Y$ the following conditions are equivalent:

(i) $Y$ is a Brauner space;

(ii) $Y$ is a complete Kelley space (i.e. every set $M \subseteq Y$ that has a closed intersection $M \cap K$ with any compact set $K \subseteq Y$, is closed in $Y$ ) and has a countable fundamental system of compact sets $K_{n}$ : for each compact set $T \subset Y$ there exists $n \in \mathbb{N}^{1}$ such that $T \subseteq K_{n}$;

(iii) $Y$ is a stereotype space and has a countable fundamental system of compact sets $K_{n}$ : for each compact set $T \subset Y$ there exists $n \in \mathbb{N}$ such that $T \subseteq K_{n}$;

\footnotetext{
${ }^{1}$ Everywhere in our paper $\mathbb{N}$ means the set of non-negative integers: $\mathbb{N}:=\{0,1,2,3, \ldots\}$.
} 
(iv) $Y$ is a stereotype space and has a countable exhausting system of compact sets $K_{n}$ : $\bigcup_{n=1}^{\infty} K_{n}=Y$.

Proof. The countable fundamental system of compact sets in $Y$ is the system of polars $K_{n}={ }^{\circ} U_{n}$ of a local base $U_{n}$ in the dual Fréchet space $Y^{\star}$. Modulo this remark all the statements in proposition 0.1 are obvious, except one - that a Brauner space $Y$ is always a Kelley space. This result belongs to K. Brauner and is deduced in his paper [7] from the Banach-Dieudonné theorem (see [17]).

Corollary 0.1. If $Y$ is a Brauner space with the fundamental system of compact sets $K_{n}$, then any linear mapping $\varphi: Y \rightarrow Z$ into a locally convex space $Z$ is continuous if and only if it is continuous on each compact set $K_{n}$.

Example 0.2 (Banach spaces and Smith spaces). These are special cases of Fréchet spaces and Brauner spaces. If $X$ is a Banach space, then $X$ and $Y=X^{\star}$ are stereotype spaces [31]. The polar $K=B^{\circ}$ of a ball $B$ in $Y$ is a universal compact set in $Y$, i.e. a compact set that swallows any other compact set $T$ in $Y$. The spaces $Y=X^{\star}$ dual to Banach spaces $X$ (in the sense of our definition) were originally considered by M. F. Smith in 31] - that is why we call them Smith spaces. Their characteristic properties are listed in the following proposition:

Proposition 0.2. For a locally convex space $Y$ the following conditions are equivalent:

(i) $Y$ is a Smith space;

(ii) $Y$ is a complete Kelley space and has a universal compact set $K$ : for any compact set $T \subset X$ there exists $\lambda \in \mathbb{C}$ such that $T \subseteq \lambda K$;

(iii) $Y$ is a stereotype space with a universal compact set;

(iv) $Y$ is a stereotype space with a compact barrel.

Corollary 0.2. If $Y$ is a Smith space with a universal compact set $K$, then a linear mapping $\varphi: Y \rightarrow Z$ into a locally compact space $Z$ is continuous if and only if it is continuous on $K$.

The connections between the spaces of Fréchet, Brauner, Banach and Smith are illustrated in the following diagram (where turnover corresponds to the passage to the dual class):

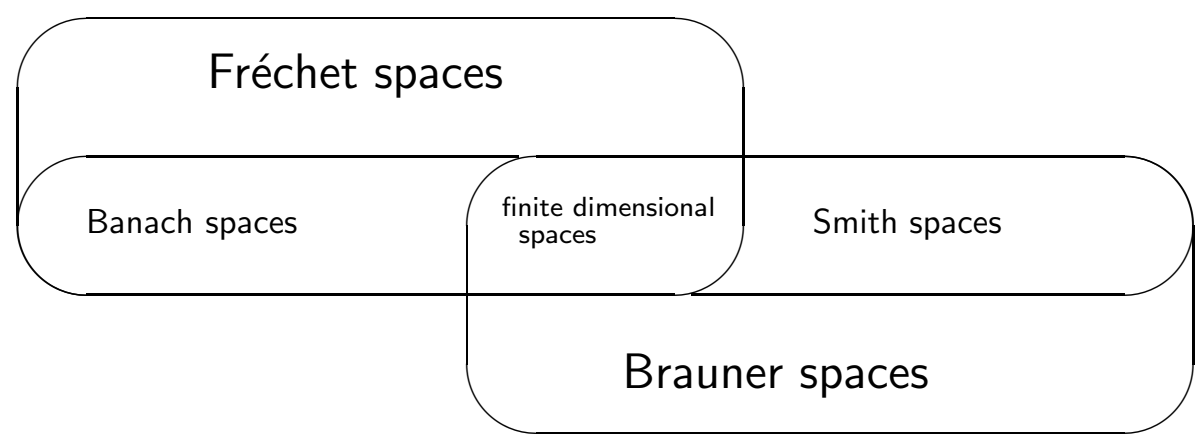

\section{(b) Smith space generated by a compact set}

Let $X$ be a stereotype space and $K$ an absolutely convex compact set in $X$. We denote by $\mathbb{C} K$ the linear subspace in $X$, generated by the set $K$ :

$$
\mathbb{C} K=\bigcup_{\lambda>0} \lambda K
$$

Endow $\mathbb{C} K$ by the Kelley topology generated by compact sets $\lambda K$ : a set $M \subseteq \mathbb{C} K$ is considered closed in $\mathbb{C} K$, if its intersection $M \cap \lambda K$ with any compact set $\lambda K$ is closed in $X$ (or, equivalently, in $\lambda K$ ). 
Theorem 0.2. The Kelley topology on $\mathbb{C} K$, generated by compact sets $\lambda K$, is a unique topology on $\mathbb{C} K$, which turns $\mathbb{C} K$ into a Smith space with the universal compact set $K$.

Proof. Denote by $(\mathbb{C} K)^{\prime}$ the set of all linear functionals on $\mathbb{C} K$, continuous on the compact set $K$ :

$$
f \in(\mathbb{C} K)^{\prime} \Longleftrightarrow f:\left.\mathbb{C} K \rightarrow \mathbb{C} \quad \& \quad f\right|_{K} \in C(K)
$$

Clearly, $(\mathbb{C} K)^{\prime}$ is a Banach space with respect to the norm

$$
\|f\|=\max _{x \in K}|f(x)|
$$

(formally this turns $(\mathbb{C} K)^{\prime}$ into a closed subspace in $\left.C(K)\right)$. Note that functionals $f \in(\mathbb{C} K)^{\prime}$ separate points in $K$, because those of them who are restrictions on $\mathbb{C} K$ of functionals from $g \in X^{\star}$ already possess this property:

$$
\forall x, y \in K \quad\left(x \neq y \quad \Longrightarrow \quad \exists g \in X^{\star} \quad g(x) \neq g(y)\right)
$$

This means that the weak topology $\sigma$ on $K$, generated by functionals $f \in(\mathbb{C} K)^{\prime}$, coincides with the initial topology $\tau$ of this compact set (because $\sigma$ is Hausdorff and is majorized by $\tau$ ). This implies in its turn that the topology of the space $(\mathbb{C} K)^{\prime}$ is the topology of uniform convergence on $(\mathbb{C} K)^{\prime}$-weak absolutely convex compact sets of the form $\lambda K$ (these sets form a saturated system in $\mathbb{C} K$ ). Hence by the Mackey-Arens theorem [32], the system $\left((\mathbb{C} K)^{\prime}\right)^{\star}$ of linear continuous functionals on $(\mathbb{C} K)^{\prime}$ coincides with $\mathbb{C} K$ :

$$
\mathbb{C} K=\left((\mathbb{C} K)^{\prime}\right)^{\star}
$$

We see that $\mathbb{C} K$ can be identified with the space of linear continuous functionals on $(\mathbb{C} K)^{\prime}$. As a corollary, $\mathbb{C} K$ can be endowed with the topology of dual space (in stereotype sense) to the Banach space $(\mathbb{C} K)^{\prime}$ :

$$
\mathbb{C} K \cong\left((\mathbb{C} K)^{\prime}\right)^{\star}
$$

This topology turns $\mathbb{C} K$ into a Smith space, and, by Proposition 0.2 , it coincides with the Kelley topology on $\mathbb{C} K$, generated by compact sets $\lambda K$.

Let us denote this topology on $\mathbb{C} K$ by $\varkappa$, and show that it is a unique topology under which $\mathbb{C} K$ is a Smith space with the universal compact set $K$. Indeed, if $\rho$ is another topology on $\mathbb{C} K$ with the same property, then the identity mapping

$$
(\mathbb{C} K)_{\rho} \rightarrow(\mathbb{C} K)_{\varkappa}
$$

is continuous on $K$ (since it preserves the topology on $K$ ), hence by Corollary 0.2 , it is a continuous mapping of Smith spaces. In the same way, the inverse mapping

$$
(\mathbb{C} K)_{\varkappa} \rightarrow(\mathbb{C} K)_{\rho}
$$

is continuous, and this means that the topologies $\varkappa$ and $\rho$ coincide.

Corollary 0.3. The topology of the space $\mathbb{C} K$ can be equivalently described as the topology of uniform convergence on sequences of functionals $\left\{f_{k}\right\} \subset(\mathbb{C} K)^{\prime}$ tending to zero, i.e. as the topology generated by seminorms of the form:

$$
p_{\left\{f_{k}\right\}}(x)=\sup _{k \in \mathbb{N}}\left|f_{n}(x)\right|
$$

where $f_{k}$ is a sequence of linear functionals on $\mathbb{C} K$, continuous on $K$, and such that $\max _{t \in K}\left|f_{k}(t)\right| \underset{k \rightarrow \infty}{\longrightarrow} 0$.

Proposition 0.3. For any two absolutely convex compact sets $K, L \subseteq X$ such that

$$
K \subseteq L
$$

the natural imbedding of the Smith spaces which they generate

$$
\iota_{K}^{L}: \mathbb{C} K \rightarrow \mathbb{C} L
$$

is a continuous mapping.

Proof. The mapping $\iota_{K}^{L}$ is continuous on the compact set $K$, hence, by Corollary 0.2 , it is continuous on all $\mathbb{C} K$. 


\section{(c) Brauner spaces generated by an expanding sequence of compact sets}

A sequence of absolutely convex compact sets $K_{n}$ in a stereotype space $X$ will be called expanding, if

$$
\forall n \in \mathbb{N} \quad K_{n}+K_{n} \subseteq K_{n+1}
$$

For every such sequence the set

$$
\bigcup_{n=1}^{\infty} \mathbb{C} K_{n}=\bigcup_{n \in \mathbb{N}, \lambda \in \mathbb{C}} \lambda K_{n}=\bigcup_{n \in \mathbb{N}} K_{n}
$$

is a subspace in the vector space $X$. We endow it with the Kelley topology generated by compact sets $K_{n}$ : a set $M \subseteq \bigcup_{n=1}^{\infty} K_{n}$ is considered closed in $\bigcup_{n=1}^{\infty} K_{n}$, if its intersection $M \cap K_{n}$ with any compact set $K_{n}$ is closed in $X$ (and equivalently, in $K_{n}$ ). The following proposition is proved similarly with Theorem 0.2 .

Theorem 0.3. The Kelley topology on $\bigcup_{n=1}^{\infty} K_{n}$, generated by compact sets $K_{n}$, is a unique topology on $\bigcup_{n=1}^{\infty} K_{n}$, under which this space is a Brauner space with the fundamental sequence of compact sets $\left\{K_{n}\right\}$.

Corollary 0.4. The topology of the space $\bigcup_{n=1}^{\infty} K_{n}$ can be equivalently described as the topology of uniform convergence on sequences of functionals $\left\{f_{k}\right\} \subset\left(\bigcup_{n=1}^{\infty} K_{n}\right)^{\prime}$ tending to zero, i.e. as the topology generated by seminorms of the form:

$$
p_{\left\{f_{k}\right\}}(x)=\sup _{k \in \mathbb{N}}\left|f_{k}(x)\right|
$$

where $f_{k}$ is an arbitrary sequence of linear functionals on $\bigcup_{n=1}^{\infty} K_{n}$, continuous on each $K_{n}$, such that $\forall n \max _{t \in K_{n}}\left|f_{k}(t)\right| \underset{k \rightarrow \infty}{\longrightarrow} 0$.

\section{(d) Projective Banach systems and injective Smith systems}

Let $X$ be a locally convex space. A standard construction in the theory of topological vector spaces assigns to each absolutely convex neighborhood of zero $U$ in $X$ a Banach space, which it is convenient to denote by $X / U$ and to call a quotient space of $X$ over the neighborhood of zero $U$. It is defined as follows. First, we consider the set

$$
\operatorname{Ker} U=\bigcap_{\varepsilon>0} \varepsilon \cdot U,
$$

which is called kernel of the neighborhood of zero $U$ - this is a closed subspace in $X$, since $U$ is absolutely convex. Then we construct the quotient space $X / \operatorname{Ker} U$, and endow it with the topology of normed space with $U+\operatorname{Ker} U$ as unit ball (this topology in general is weaker than the usual topology of quotient space on $X / \operatorname{Ker} U)$. This normed space $X / \operatorname{Ker} U$ is usually not complete. Its completion is declared the final result:

$$
X / U:=(X / \operatorname{Ker} U)^{\mathbf{}}
$$

(here $\boldsymbol{\nabla}$ means completion).

Theorem 0.4. Let $X$ be a stereotype space. For any absolutely convex compact set $K \subseteq X$ the Smith space it generates, $\mathbb{C} K$, is connected with the quotient space $X^{\star} / K^{\circ}$ of the dual space $X^{\star}$ with respect to the neighborhood of zero $K^{\circ}$ through the formula

$$
(\mathbb{C} K)^{\star} \cong X^{\star} / K^{\circ}
$$


Let $X$ be a stereotype space and let $\mathcal{K}$ be a expanding system of absolutely convex compact set in $X$, i.e. a system satisfying the following condition:

$$
\forall K, L \in \mathcal{K} \quad \exists M \in \mathcal{K} \quad K \cup L \subseteq M
$$

By Proposition 0.3 for any compact sets $K, L \in \mathcal{K}$ such that $K \subseteq L$, the Smith spaces they generate are connected through a natural linear continuous mapping $\iota_{K}^{L}: \mathbb{C} K \rightarrow \mathbb{C} L$. Since, obviously, for any three compact sets $K \subseteq L \subseteq M$ the corresponding mappings are connected through the equality

$$
\iota_{L}^{M} \circ \iota_{K}^{L}=\iota_{K}^{M},
$$

the arising system of mappings $\left\{\iota_{K}^{L} ; K, L \in \mathcal{K}: K \subseteq L\right\}$ is an injective system in the category Ste of stereotype spaces. Like any other injective system in $\mathfrak{S} \mathfrak{t} \mathfrak{e}$, it has a limit - this is the pseudocompletion of its locally convex injective limit [1, Theorem 4.21]:

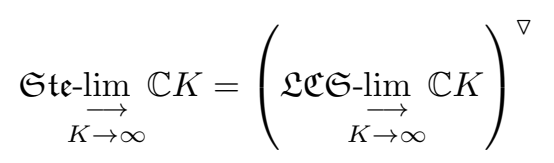

The dual construction is often used in the theory of topological vector spaces. Let $X$ be a stereotype space and suppose $\mathcal{U}$ is a decreasing system of absolutely convex neighborhoods of zero in $X$, i.e. a system satisfying the following condition:

$$
\forall U, V \in \mathcal{U} \quad \exists W \in \mathcal{U} \quad W \subseteq U \cap V
$$

For any two neighborhoods of zero $U, V \in \mathcal{U}$ such that $V \subseteq U$ the Banach spaces they generate $X / \operatorname{Ker} U$ and $X / \operatorname{Ker} V$ are connected with each other through a natural linear continuous mapping $\pi_{U}^{V}: X / \operatorname{Ker} V \rightarrow X / \operatorname{Ker} U$. If we consider three neighborhoods of zero $W \subseteq V \subseteq U$ the corresponding mappings are connected through the equality

$$
\pi_{U}^{V} \circ \pi_{V}^{W}=\pi_{U}^{W}
$$

This means that the system of mappings $\left\{\pi_{U}^{V} ; U, V \in \mathcal{U}: V \subseteq U\right\}$ is a projective system in the category $\mathfrak{S t e}$ of stereotype spaces. Its limit is the pseudosaturation of its locally convex projective limit [1, Theorem 4.21]:

$$
\underset{0 \leftarrow U}{\mathfrak{S t e}-\lim _{0 \leftarrow U}} X / U=\left(\underset{0 \leftarrow U}{\mathfrak{L} \mathfrak{C} \mathfrak{S}-\lim _{0 \leftarrow U}^{\longleftarrow}} X / U\right)^{\star}
$$

From (0.4) we have:

Theorem 0.5. If $\mathcal{K}$ is an expanding system of absolutely convex compact sets in a stereotype space $X$, then the system of polars $\mathcal{U}=\left\{K^{\circ} ; K \in \mathcal{K}\right\}$ is a decreasing system of absolutely convex neighborhoods of zero in the dual space $X^{\star}$. The limits of these systems are dual to each other:

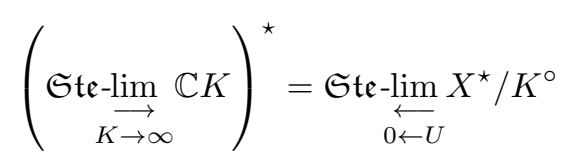

Example 0.3. Let $\mathcal{K}=\mathcal{K}(X)$ be a system of all absolutely convex compact sets in $X$. Then the limit of the injective system $\left\{\iota_{K}^{L} ; K, L \in \mathcal{K}: K \subseteq L\right\}$ in the category of stereotype spaces is the saturation ${ }^{2}$ of the space $X$ :

$$
\underset{K \rightarrow \infty}{\longrightarrow} \mathbb{C t e -} \lim _{K \rightarrow \infty} \mathbb{C} K=X^{\boldsymbol{\Delta}}
$$

\footnotetext{
${ }^{2}$ Saturation $X^{\boldsymbol{\Lambda}}$ of a locally convex space $X$ was defined in 11 1.2].
} 
Example 0.4. Dually, if $\mathcal{U}=\mathcal{U}(X)$ is the set of all absolutely convex neighborhoods of zero in $X$, then the limit of the projective system $\left\{\pi_{U}^{V} ; U, V \in \mathcal{U}: V \subseteq U\right\}$ in the category of stereotype spaces is the completion of the space $X$ :

$$
\underset{0 \mathfrak{S t e}-\lim _{0 \leftarrow U}^{\longleftarrow}}{\leftarrow \leftarrow U}=X^{\mathbf{\nabla}}
$$

Theorem 0.6. If $K_{n}$ is an expanding sequence of absolutely convex compact sets in a stereotype space $X$, then the limit of the injective system $\mathbb{C} K_{n}$ in the category of stereotype spaces coincides with locally convex limit of this system and with the Brauner space generated by the sequence $K_{n}$ :

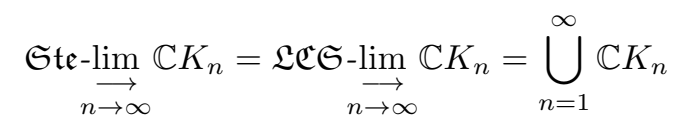

\section{(e) Banach representation of a Smith space}

If $X$ is a Banach space (with the norm $\|\cdot\|_{X}$ ), then let us denote by $X^{*}$ its dual Banach space in the usual sense, i.e. the space of linear continuous functionals on $X$ with the norm

$$
\|f\|_{X^{*}}=\sup _{\|x\|_{X} \leqslant 1}|f(x)|
$$

If $\varphi: X \rightarrow Y$ is a continuous linear mapping of Banach spaces, then the symbol $\varphi^{*}: Y^{*} \rightarrow X^{*}$ denotes the dual mapping:

$$
\varphi^{*}(f)=f \circ \varphi, \quad f \in Y^{*}
$$

The natural mapping from $X$ into $X^{* *}$ will be denoted by $s_{X}$ :

$$
s_{X}: X \rightarrow X^{* *}
$$

Let $Y$ be a Smith space with the universal compact set $T$. Denote by $Y^{\mathrm{B}}$ the normed space with $Y$ as support and $T$ as unit ball.

Theorem 0.7. For any Smith space $Y$

$$
Y^{\mathrm{B}} \cong\left(Y^{\star}\right)^{*}
$$

hence, $Y^{\mathrm{B}}$ is a (complete and so a) Banach space.

Proof. Consider the space $X=Y^{\star}$. Then $Y$ becomes a space of linear continuous functionals on a Banach space $X$ with the topology of uniform convergence on compact sets in $X$. The universal compact $T$ in $Y$ becomes a polar of the unit ball $B$ in $X$. If we endow $Y$ with the topology, where $T$ is a unit ball, this is the same as if we endow $Y$ with the topology of the normed space, dual to $X: Y^{\mathrm{B}}=X^{*}=\left(Y^{\star}\right)^{*}$. Since the (Banach) dual to a Banach space is always a Banach space, $Y^{\mathrm{B}}$ must be a Banach space as well.

We call the space $Y^{\mathrm{B}}$ Banach representation of the Smith space $Y$. Note that the natural mapping

$$
\iota_{Y}: Y^{\mathrm{B}} \rightarrow Y, \quad \iota_{Y}(y)=y
$$

is universal in the following sense: for any Banach space $Z$ and for any linear continuous mapping $\varphi: Z \rightarrow Y$ there exists a unique linear continuous mapping $\chi: Z \rightarrow Y^{\mathrm{B}}$ such that the following diagram is commutative:

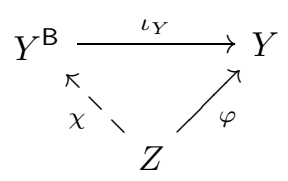


If $\varphi: X \rightarrow Y$ is a linear continuous mapping of Smith spaces, then it turns the universal compact set $S$ in $X$ into a compact set $\varphi(S)$ in $Y$, which, like any other compact set, is contained in some homothety of the universal compact set $T$ in $Y$ :

$$
\varphi(S) \subseteq \lambda T, \quad \lambda>0
$$

This means that the mapping $\varphi$, being considered as the mapping between the corresponding Banach representations $X^{\mathrm{B}} \rightarrow Y^{\mathrm{B}}$, is also continuous. We shall denote this mapping by the symbol $\varphi^{\mathrm{B}}$

$$
\varphi^{\mathrm{B}}: X^{\mathrm{B}} \rightarrow Y^{\mathrm{B}}
$$

and call it Banach representation of the mapping $\varphi$. Obviously,

$$
\varphi^{\mathrm{B}} \cong\left(\varphi^{\star}\right)^{*}
$$

and the following diagram is commutative

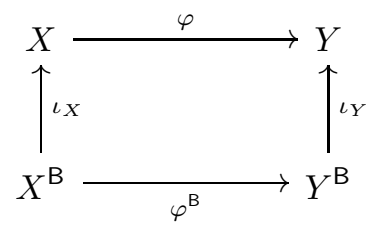

\section{(f) Injective systems of Banach spaces, generated by compact sets}

The following construction is often used in the theory of topological vector spaces. If $B$ is a bounded absolutely convex closed set in a locally convex space $X$, then $X_{B}$ denotes the space $\bigcup_{\lambda>0} \lambda B$, endowed with the topology of normed space with the unit ball $B$. If $X_{B}$ turns out to be complete (i.e. a Banach) space, then the set $B$ is called a Banach disk. From theorem 0.7 we have

Proposition 0.4. Every absolutely convex compact set $K$ in a stereotype space $X$ is a Banach disk, and the Banach space $X_{K}$ generated by $K$ is the Banach representation of the Smith space $\mathbb{C} K$ :

$$
X_{K}=(\mathbb{C} K)^{\mathrm{B}}
$$

If $\mathcal{K}$ is an expanding system of absolutely convex compact sets in a stereotype space $X$, then, as we told above, $\mathcal{K}$ generates an injective system of Smith spaces

$$
\{\mathbb{C} K\}_{K \in \mathcal{K}}, \quad \iota_{K}^{L}: \mathbb{C} K \rightarrow \mathbb{C} L \quad(K, L \in \mathcal{K}, \quad K \subseteq L) .
$$

Specialists in topological vector spaces used to replace this system of Smith spaces with the system of the corresponding Banach representations:

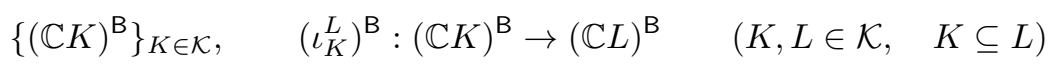

The following result shows that the limits of those systems coincide in the case when the injections $\iota_{K}^{L}: \mathbb{C} K \rightarrow \mathbb{C} L$ are compact mappings.

Theorem 0.8. Let $\mathcal{K}$ be an expanding system of absolutely convex compact sets in a stereotype space $X$. Then the following conditions are equivalent:

(i) for any compact set $K \in \mathcal{K}$ there is a compact set $L \in \mathcal{K}$ such that $K \subseteq L$ and the mapping of Smith spaces $\iota_{K}^{L}: \mathbb{C} K \rightarrow \mathbb{C} L$ is compact,

(ii) for any compact set $K \in \mathcal{K}$ there is a compact set $L \in \mathcal{K}$ such that $K \subseteq L$ and the mapping of Banach spaces $\left(\iota_{K}^{L}\right)^{\mathrm{B}}:(\mathbb{C} K)^{\mathrm{B}} \rightarrow(\mathbb{C} L)^{\mathrm{B}}$ is compact. 
If these conditions hold then the locally convex injective limits of the systems $\{\mathbb{C} K\}_{K \in \mathcal{K}}$ and $\left\{(\mathbb{C} K)^{\mathrm{B}}\right\}_{K \in \mathcal{K}}$ coincide,

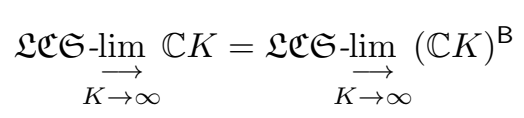

and the same is true for their stereotype injective limits:

$$
\underset{K \rightarrow \infty}{\mathfrak{S t e}-\lim _{K \rightarrow \infty}} \mathbb{C} K=\mathfrak{S t e}-\lim _{K \rightarrow \infty}(\mathbb{C} K)^{\mathrm{B}}
$$

If in addition to (i)-(ii) the system $\mathcal{K}$ is countable (or contains a countable cofinal subsystem), then those four limits coincide,

$$
\underset{K \rightarrow \mathfrak{t e}-\lim _{K \rightarrow \infty}}{\longrightarrow} K=\mathfrak{L C S}-\underset{K \rightarrow \infty}{\longrightarrow} \mathbb{C} K=\underset{K \rightarrow \infty}{\mathfrak{L} \mathfrak{C}-\lim _{K \rightarrow \infty}}(\mathbb{C} K)^{\mathrm{B}}=\mathfrak{S t e}-\underset{K \rightarrow \infty}{\longrightarrow}(\mathbb{C} K)^{\mathrm{B}}
$$

and define a Brauner space.

We shall premise the proof of this theorem by several auxiliary propositions.

First we note that by compact mapping of stereotype spaces we mean what is usually meant, i.e. a linear continuous mapping $\varphi: X \rightarrow Y$ such that

$$
\varphi(U) \subseteq T
$$

for some neighborhood of zero $U \subseteq X$ and some compact set $T \subseteq Y$.

Proposition 0.5. Let $X$ and $Y$ be Smith spaces and $\varphi: X \rightarrow Y$ a linear continuous mapping. The following conditions are equivalent:

(i) $\varphi: X \rightarrow Y$ is a compact mapping;

(ii) $\varphi^{\star}: Y^{\star} \rightarrow X^{\star}$ is a compact mapping;

(iii) $\varphi^{\mathrm{B}}: X^{\mathrm{B}} \rightarrow Y^{\mathrm{B}}$ is a compact mapping.

Proof. The equivalence (i) $\Leftrightarrow($ ii) is obvious, and (ii) $\Leftrightarrow($ iii) follows from (0.8) and a classical result on compact mappings [30, Theorem 4.19].

Proposition 0.6. If $\varphi: X \rightarrow Y$ is a compact mapping of Banach spaces, then its (Banach) second dual mapping $\varphi^{* *}: X^{* *} \rightarrow Y^{* *}$ turns $X^{* *}$ into $Y$ :

$$
\varphi^{* *}\left(X^{* *}\right) \subseteq Y
$$

Proof. Let $B$ be unit ball in $X$ and $T$ a compact set in $Y$ such that

$$
\varphi(B) \subseteq T
$$

By the bipolar theorem, $B$ is $X^{*}$-weakly dense in the unit ball $B^{\circ \circ}$ of the space $X^{* *}$. Hence for each $z \in B^{\circ \circ}$ one can choose a net $z_{i} \in B$ tending to $z X^{*}$-weakly

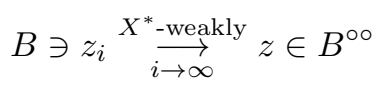

Since $\varphi$, like any weakly compact operator, turns $X^{*}$-weak Cauchy nets into 'strong' Cauchy nets, we obtain that $\varphi\left(z_{i}\right)$ is a Cauchy net in the compact set $T$, hence it tends to some $y \in T$.

$$
\varphi\left(z_{i}\right) \underset{i \rightarrow \infty}{\stackrel{Y}{\longrightarrow}} y
$$


From this we have:

$$
\forall g \in Y^{*} \quad \varphi^{* *}\left(z_{i}\right)(g)=\varphi *(g)\left(z_{i}\right)=g\left(\varphi\left(z_{i}\right)\right) \underset{i \rightarrow \infty}{\longrightarrow} g(y)=\mathrm{i}_{Y}(y)(g)
$$

(where $\mathrm{i}_{Y}: Y \rightarrow Y^{* *}$ is a natural embedding).

On the other hand from (0.13) we also obtain that

$$
\forall f \in X^{*} \quad f\left(z_{i}\right) \underset{i \rightarrow \infty}{\longrightarrow} f(z)
$$

and, in particular, this must be true for functionals $f=\varphi *(g)=g \circ \varphi$, where $g \in Y^{*}$ :

$$
\forall g \in Y^{*} \quad \varphi^{* *}\left(z_{i}\right)(g)=\varphi *(g)\left(z_{i}\right) \underset{i \rightarrow \infty}{\longrightarrow} \varphi *(g)(z)=\varphi^{* *}(z)(g)
$$

From (0.14) and (0.15) we have

$$
\varphi^{* *}(z)=y
$$

I.e., $\varphi^{* *}(z) \in Y$. This holds for each point $z \in B^{\circ \circ}$, so 0.12 must be true.

Proposition 0.7. Let $\varphi: X \rightarrow Y$ be a linear continuous mapping of stereotype spaces. For any absolutely convex closed set $V \subseteq Y$ the following formula holds:

$$
\varphi^{-1}(V)=\circ\left(\varphi^{\star}\left(V^{\circ}\right)\right)
$$

Proof.

$$
\begin{aligned}
x \in^{\circ}\left(\varphi^{\star}\left(V^{\circ}\right)\right) \Longleftrightarrow \forall g \in V^{\circ}\left|\varphi^{\star}(g)(x)\right|=|g(\varphi(x))| \leqslant 1 & \Longleftrightarrow \\
& \Longleftrightarrow \varphi(x) \in{ }^{\circ}\left(V^{\circ}\right)=V \quad \Longleftrightarrow x \in \varphi^{-1}(V)
\end{aligned}
$$

Proposition 0.8. Let $\sigma: X \rightarrow Y$ be a compact mapping of Smith spaces, and $V$ an absolutely convex closed neighborhood of zero in the Banach representation $Y^{\mathrm{B}}$ of the space $Y$. Then its preimage $\left(\sigma^{\mathrm{B}}\right)^{-1}(V)=\sigma^{-1}(V)$ is a neighborhood of zero in the space $X$ (and not only in its Banach representation $\left.X^{\mathrm{B}}\right)$.

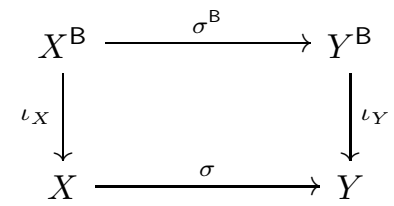

Proof. By formula (0.8), we can consider $\sigma^{\mathrm{B}}$ as a mapping of dual Banach spaces for $X^{\star}$ and $Y^{\star}$ :

$$
\sigma^{\mathrm{B}}=\left(\sigma^{\star}\right)^{*}:\left(X^{\star}\right)^{*} \rightarrow\left(Y^{\star}\right)^{*}
$$

Let us consider the Banach dual mapping $\left(\sigma^{\mathrm{B}}\right)^{*}=\left(\sigma^{\star}\right)^{* *}:\left(Y^{\star}\right)^{* *} \rightarrow\left(X^{\star}\right)^{* *}$. Since $\sigma^{\star}$ is compact, from Proposition 0.6 it follows that

$$
\left(\sigma^{\star}\right)^{* *}\left(\left(Y^{\star}\right)^{* *}\right) \subseteq X^{\star}
$$

This can be illustrated by the diagram

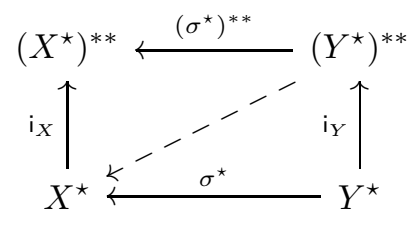


Now we have:

$V$ is a neighborhood of zero in $Y^{\mathrm{B}}=\left(Y^{\star}\right)^{*}$

$\Downarrow$

$V^{\circ}$ is a bounded set in $\left(Y^{\star}\right)^{* *}$

$\Downarrow$

$\left(\sigma^{\star}\right)^{* *}\left(V^{\circ}\right)$ is a totally bounded set in $\left(X^{\star}\right)^{* *}$

(since $\left(\sigma^{\star}\right)^{* *}$ is a compact mapping) \& $\left(\sigma^{\star}\right)^{* *}\left(V^{\circ}\right) \subseteq X^{\star}$

$\Downarrow$

$\left(\sigma^{\star}\right)^{* *}\left(V^{\circ}\right)$ is a totally bounded set in $X^{\star}$

$\Downarrow$

$\left(\sigma^{\mathrm{B}}\right)^{-1}(V)=(\underline{0.16})={ }^{\circ}\left(\left(\sigma^{\mathrm{B}}\right)^{*}\left(V^{\circ}\right)\right)={ }^{\circ}\left(\left(\sigma^{\star}\right)^{* *}\left(V^{\circ}\right)\right)$ is a neighborhood of zero in $X$

Proof of Theorem 0.8 . The equivalence of (i) and (ii) immediately follows from Propositions 0.5. After that formula (0.9) follows from Proposition 0.8 if $U$ is an absolutely convex neighborhood of zero in $\mathfrak{L C S}-\lim _{K}(\mathbb{C} K)^{\mathrm{B}}$, i.e. $U=\left\{U_{K} ; K \in \mathcal{K}\right\}$ is a system of neighborhoods of zeroes in the spaces $(\mathbb{C} K)^{\mathrm{B}}$, $\longrightarrow \rightarrow \infty$

satisfying the condition

$$
\forall K, L \in \mathcal{K} \quad\left(K \subseteq L \quad \Longrightarrow \quad U_{K}=\left(\iota_{K}^{L}\right)^{-1}\left(U_{L}\right)\right)
$$

then from Proposition 0.8 it follows that all those neighborhoods are neighborhoods of zero in the spaces $\mathbb{C} K$. This proves the continuity of the mapping

$$
\underset{K \rightarrow \infty}{\mathfrak{L C S}-\lim _{K \rightarrow \infty}} \mathbb{C} K \rightarrow \underset{K \rightarrow \infty}{\mathfrak{L C S}-\lim }(\mathbb{C} K)^{\mathrm{B}}
$$

The continuity of the inverse mapping is obvious. Thus, the topologies in those spaces coincide. In other words, those limits coincide, and we obtain (0.9). This implies in its turn (0.10). Finally if $\mathcal{K}$ is countable, then by Theorem 0.6 the locally convex limits coincide with stereotype limits, and we have (0.11) .

\section{(g) Nuclear stereotype spaces}

A mapping of stereotype spaces $\varphi: X \rightarrow Y$ we shall call nuclear, if it satisfies the following four equivalent conditions:

(i) there exist two sequences

$$
f_{n} \underset{n \rightarrow \infty}{\stackrel{X^{\star}}{\rightarrow}} 0, \quad b_{n} \underset{n \rightarrow \infty}{\stackrel{Y}{\rightarrow}} 0, \quad \lambda_{n} \geqslant 0, \quad \sum_{n=1}^{\infty} \lambda_{n}<\infty
$$

such that

$$
\varphi(x)=\sum_{n=1}^{\infty} \lambda_{n} \cdot f_{n}(x) \cdot b_{n}, \quad x \in X ;
$$

(ii) there exist two totally bounded sequences $\left\{f_{n}\right\}$ in $X^{\star}$ and $\left\{b_{n}\right\}$ in $Y$, and a number sequence $\lambda_{n} \geqslant 0, \sum_{n=1}^{\infty} \lambda_{n}<\infty$ such that (0.17) holds; 
(iii) there exist two bounded sequences $\left\{f_{n}\right\}$ in $X^{\star}$, and $\left\{b_{n}\right\}$ in $Y$, and a number sequence $\lambda_{n} \geqslant 0$, $\sum_{n=1}^{\infty} \lambda_{n}<\infty$ such that (0.17) holds;

(iv) there exist a sequence of functionals $\left\{f_{n}\right\} \subseteq X^{\star}$, equicontinuous on $X$, and a sequence of vectors $\left\{b_{n}\right\}$ in some Banach disk $B \subset Y$, and a number sequence $\lambda_{n} \geqslant 0, \sum_{n=1}^{\infty} \lambda_{n}<\infty$ such that (0.17) holds.

Clearly, a nuclear mapping is always continuous.

Proof. The implications (i) $\Rightarrow$ (ii) $\Rightarrow$ (iii) are obvious. Let us prove the implication (iii) $\Rightarrow$ (i). Suppose (iii) is true, i.e. the sequences $\left\{f_{n}\right\} \subset X^{\star}$ and $\left\{b_{n}\right\} \subset Y$ in the formula (0.17) are just bounded. Let us choose a number sequences $\sigma_{n}>0$ such that

$$
\sigma_{n} \underset{n \rightarrow \infty}{\longrightarrow}+\infty, \quad \sum_{n=1}^{\infty} \lambda_{n} \cdot \sigma_{n}<\infty
$$

(we can denote $\sum_{n=1}^{\infty} \lambda_{n}=C$ for this, and then divide the sequence $\lambda_{n}$ into blocks $\sum_{n_{k}<n \leqslant n_{k+1}} \lambda_{n} \leqslant \frac{C}{2^{k}}$, $k \geqslant 0, n_{0}=0$, and put $\sigma_{n}=k+1$ for $\left.n_{k}<n \leqslant n_{k+1}\right)$. Then we can replace $f_{n}, b_{n}, \lambda_{n}$ with

$$
\widetilde{f_{n}}:=\frac{f_{n}}{\sqrt{\sigma_{n}}}, \quad \widetilde{b_{n}}:=\frac{b_{n}}{\sqrt{\sigma_{n}}}, \quad \widetilde{\lambda_{n}}:=\lambda_{n} \cdot \sigma_{n},
$$

because

$$
\sum_{n=1}^{\infty} \widetilde{\lambda_{n}} \cdot \widetilde{f_{n}}(x) \cdot \widetilde{b_{n}}=\sum_{n=1}^{\infty} \lambda_{n} \cdot \sigma_{n} \cdot \frac{f_{n}(x)}{\sqrt{\sigma_{n}}} \cdot \frac{b_{n}}{\sqrt{\sigma_{n}}}=\sum_{n=1}^{\infty} \lambda_{n} \cdot f_{n}(x) \cdot b_{n}=\varphi(x)
$$

And since $f_{n}$ and $b_{n}$ are bounded, then $\widetilde{f_{n}}$ and $\widetilde{b_{n}}$ tend to zero

$$
\widetilde{f_{n}} \underset{n \rightarrow \infty}{\longrightarrow} 0, \quad \widetilde{b_{n}} \underset{n \rightarrow \infty}{\longrightarrow} 0, \quad \sum_{n=1}^{\infty} \widetilde{\lambda_{n}}<\infty
$$

i.e. (i) holds.

It remains now to show that the conditions (i)-(iii) are equivalent to (iv), i.e. the standard definition of nuclear mapping [32, 17. The implication (iv) $\Rightarrow$ (iii) is obvious. On the other hand, the implication (ii) $\Rightarrow$ (iv) holds: from (ii) it follows that the sequence of functionals $\left\{f_{n}\right\}$, being totally bounded in $X^{\star}$, is equicontinuous on $X$ by [1, Theorem 2.5], and the sequence $\left\{b_{n}\right\}$ belong to Banach disk $T=\overline{\operatorname{absconv}}\left\{b_{n}\right\}$ by theorem 0.4 . This is exactly (iv).

Theorem 0.9. A linear continuous mapping between stereotype spaces $\varphi: X \rightarrow Y$ is nuclear if and only if its dual mapping $\varphi^{\star}: Y^{\star} \rightarrow X^{\star}$ is nuclear.

Let us call a mapping of stereotype spaces $\varphi: X \rightarrow Y$ quasinuclear, if for any compact set $T \subset Y^{\star}$ there exist a sequence of functionals $\left\{f_{n}\right\} \subseteq X^{\star}$ equicontinuous on $X$ and a number sequence $\lambda_{n} \geqslant 0$, $\sum_{n=1}^{\infty} \lambda_{n}<\infty$ such that

$$
\max _{g \in T}|g(\varphi(x))| \leqslant \sum_{n=1}^{\infty} \lambda_{n}\left|f_{n}(x)\right|
$$

If $X$ and $Y$ are Banach spaces, then quasinuclearity of $\varphi: X \rightarrow Y$ means inequality

$$
\|\varphi(x)\|_{Y} \leqslant \sum_{n=1}^{\infty} \lambda_{n}\left|f_{n}(x)\right|
$$

where $\|\cdot\|_{Y}$ is the norm in $Y, f_{n}$ a bounded sequence of functionals on $X, \lambda_{n}$ a summable number sequence. 
Lemma 0.1. Let $\varphi: X \rightarrow Y$ be a linear continuous mapping of Banach spaces, and let $\varphi^{*}: Y^{*} \rightarrow X^{*}$ be its Banach dual mapping. Then

- if $\varphi$ is nuclear, then $\varphi^{*}$ is nuclear as well,

- if $\varphi^{*}$ is nuclear, then $\varphi$ is quasinuclear.

Proof. The first proposition here is obvious (and well-known, see [25, 3.1.8]) and implies the second one: if $\varphi^{*}: Y^{*} \rightarrow X^{*}$ is nuclear, then $\varphi^{* *}: X^{* *} \rightarrow Y^{* *}$ is also nuclear, and as a corollary, quasinuclear. From this we have that $\varphi$ is also quasinuclear, since it is a restriction (and a corestriction) of a quasinuclear mapping $\varphi^{* *}$ on the subspace $X \subseteq X^{* *}$ (and subspace $Y \subseteq Y^{* *}$ ).

As usual, we call a stereotype space $X$ nuclear [25], if every its continuous linear mapping into an arbitrary Banach space $X \rightarrow Y$ is nuclear.

Theorem 0.10 (Brauner, [7]). A Brauner space $X$ is nuclear if and only if its dual Fréchet space $X^{\star}$ is nuclear.

Theorem 0.11. Let $\mathcal{K}$ be an expanding system of absolutely convex compact sets in a stereotype space $X$. Then the following conditions are equivalent:

(i) for any compact set $K \in \mathcal{K}$ there is a compact set $L \in \mathcal{K}$ such that $K \subseteq L$ and the mapping of Smith spaces $\iota_{K}^{L}: \mathbb{C} K \rightarrow \mathbb{C} L$ is nuclear,

(ii) for any compact set $K \in \mathcal{K}$ there is a compact set $L \in \mathcal{K}$ such that $K \subseteq L$ and the mapping of Banach spaces $\left(\iota_{K}^{L}\right)^{\mathrm{B}}:(\mathbb{C} K)^{\mathrm{B}} \rightarrow(\mathbb{C} L)^{\mathrm{B}}$ is nuclear,

If these conditions hold, then the locally convex injective limits of the systems $\{\mathbb{C} K\}_{K \in \mathcal{K}}$ and $\left\{(\mathbb{C} K)^{\mathrm{B}}\right\}_{K \in \mathcal{K}}$ coincide,

$$
\underset{K \rightarrow \infty}{\mathfrak{L C S}-\lim _{K \rightarrow \infty}} \mathbb{C} K=\mathfrak{L} \mathfrak{K \rightarrow \infty}
$$

and the same is true for their stereotype injective limits:

$$
\underset{K \rightarrow \infty}{\mathfrak{S t e}-\lim _{K \rightarrow \infty}} \mathbb{C} K=\mathfrak{S t e}-\lim _{K \rightarrow \infty}(\mathbb{C} K)^{\mathrm{B}} .
$$

If in addition to (i)-(ii) the system $\mathcal{K}$ is countable (or contains a countable cofinal subsystem), then all those four limits are equal

$$
\mathfrak{S t e}-\underset{K \rightarrow \infty}{\longrightarrow} \mathbb{C} K=\mathfrak{L} \mathfrak{C} \mathfrak{S}-\lim _{K \rightarrow \infty} \mathbb{C} K=\mathfrak{L} \mathfrak{C} \mathfrak{S}-\lim _{K \rightarrow \infty}(\mathbb{C} K)^{\mathrm{B}}=\underset{K \rightarrow \infty}{\mathfrak{S} \mathfrak{e}-\lim }(\mathbb{C} K)^{\mathrm{B}}
$$

and define a nuclear Brauner space.

Proof. All statements here follow from Theorem 0.8 except the equivalence between (i) and (ii).

(i) $\Longrightarrow(\mathrm{ii})$. If $\iota_{K}^{L}$ is nuclear, then $\left(\iota_{K}^{L}\right)^{\star}$ is nuclear by Theorem 0.9. Hence $\left(\iota_{K}^{L}\right)^{\mathrm{B}}=\left(\left(\iota_{K}^{L}\right)^{\star}\right)^{*}$ is nuclear by Lemma 0.1 .

(i) $\Longrightarrow$ (ii). For a given compact set $K$ let us choose a compact set $L \supseteq K$ such that $\left(\iota_{K}^{L}\right)^{\mathrm{B}}$ is nuclear. Similarly, take a compact set $M \supseteq L$ such that $\left(\iota_{L}^{M}\right)^{\mathrm{B}}$ is nuclear. Then from nuclearity of $\left(\iota_{K}^{L}\right)^{\mathrm{B}}=\left(\left(\iota_{K}^{L}\right)^{\star}\right)^{*}$ and $\left(\iota_{L}^{M}\right)^{\mathrm{B}}=\left(\left(\iota_{L}^{M}\right)^{\star}\right)^{*}$ by Lemma 0.1 we have that $\left(\iota_{K}^{L}\right)^{\star}$ and $\left(\iota_{L}^{M}\right)^{\star}$ are quasinuclear. from this we have that $\left(\iota_{K}^{L}\right)^{\star} \circ\left(\iota_{L}^{M}\right)^{\star}$ is nuclear as a composition of quasinuclear mappings [25, 3.3.2]. Now by Theorem 0.9] $\iota_{L}^{M} \circ \iota_{K}^{L}=\iota_{K}^{M}$ becomes nuclear, as a stereotype dual mapping.

\section{(h) Spaces $\mathbb{C}^{M}$ and $\mathbb{C}_{M}$}

The spaces $\mathbb{C}^{M}$ and $\mathbb{C}_{M}$ we are talking about here are usually mentioned in textbooks on topological vector spaces as objects for exercises ([5, Chapter IV,§1, Exercise 11,13], [32, Chapter IV, Exerscise 6]). We list here some of their properties for the further references. 
Space of functions $\mathbb{C}^{M}$. Let $M$ be an arbitrary set. Denote by $\mathbb{C}^{M}$ the locally convex space of all complex-valued functions on $M$,

$$
u \in \mathbb{C}^{M} \Longleftrightarrow u: M \rightarrow \mathbb{C}
$$

with the pointwise operations, and the topology of pointwise convergence on $M$, i.e. the topology generated by seminorms

$$
|u|_{N}=\sup _{x \in N}|u(x)|
$$

where $N$ is an arbitrary finite set in $M$. We call $\mathbb{C}^{M}$ the space of functions on the set $M$. Note that $\mathbb{C}^{M}$ is isomorphic to the direct product of card $M$ copies of the field $\mathbb{C}$ :

$$
\mathbb{C}^{M} \cong \mathbb{C}^{\mathrm{card} M}
$$

As a corollary, $\mathbb{C}^{M}$ is always nuclear (since nuclearity is inherited by direct products [32, Theorem 7.4]).

Theorem 0.12. For a locally convex space $X$ over $\mathbb{C}$ the following conditions are equivalent:

(a) $X \cong \mathbb{C}^{M}$ for some set $M$;

(b) $X$ is a complete space with the weak topology ${ }^{3}$;

(c) $X$ is a space of minimal type $e^{4}$

Theorem 0.13. $\mathbb{C}^{M}$ is a Fréchet space if and only if the set $M$ is at most countable.

The space of point charges $\mathbb{C}_{M}$. Let again $M$ be an arbitrary set. Denote by $\mathbb{C}_{M}$ the set of all number families $\left\{\alpha_{x} ; x \in M\right\}$ indexed by elements of $M$ and satisfying the following finiteness condition: all the numbers $\alpha_{x}$, but a finite subfamily, vanish

$$
\alpha \in \mathbb{C}_{M} \Longleftrightarrow \alpha=\left\{\alpha_{x} ; x \in M\right\}, \quad \alpha_{x} \in \mathbb{C}, \quad \operatorname{card}\left\{x \in M: \alpha_{x} \neq 0\right\}<\infty
$$

(clearly the families $\left\{\alpha_{x} ; x \in M\right\}$ can be considered as functions $\alpha: M \rightarrow \mathbb{C}$ with finite support). The set $\mathbb{C}_{M}$ is endowed with pointwise algebraic operations (sum and multiplication by a scalar) and a topology generated by seminorms

$$
|\alpha|_{r}=\sup _{|u| \leqslant r}|\langle u, \alpha\rangle|=\sum_{x \in G} r(x) \cdot\left|\alpha_{x}\right|
$$

where $r: G \rightarrow \mathbb{R}_{+}$is an arbitrary nonnegative function on $M$. We call $\mathbb{C}_{M}$ the space of point charges on the set $M$ (and its elements - point charges on $M$ ). We can note that $\mathbb{C}_{M}$ is isomorphic to a locally convex direct sum of card $M$ copies of the field $\mathbb{C}$ :

$$
\mathbb{C}_{M} \cong \mathbb{C}_{\text {card } M}
$$

As a corollary, $\mathbb{C}_{M}$ is nuclear if and only if $M$ is at most countable (nuclearity is inherited by countable direct sums [32, Theorem 7.4], and if $M$ is not countable, then the imbedding $\mathbb{C}_{M} \rightarrow \ell_{1}(M)$ is not a nuclear mapping).

Theorem 0.14. For a locally convex space $X$ over the field $\mathbb{C}$ the following conditions are equivalent:

(a) $X \cong \mathbb{C}_{M}$ for some set $M$;

(b) $X$ is a cocomplete 5 Mackey space, where every compact set is finite-dimensional;

\footnotetext{
${ }^{3} \mathrm{~A}$ locally convex space $X$ is called a space with the weak topology, if its topology is generated by the seminorms of the form $|x|_{f}=|f(x)|$, where $f$ are linear continuous functionals on $X$.

${ }^{4} \mathrm{~A}$ locally convex space $X$ is called a space of minimal type, if there is no weaker Hausdorff locally convex topology on $X$.

${ }^{5}$ We say that a locally convex space $X$ is co-complete, if every linear functional $f: X \rightarrow \mathbb{C}$ which is continuous on every compact set $K \subset X$, is continuous on $X$.
} 
(c) topology of $X$ is maximal in the class of locally convex topologies on $X$ (i.e. there is no stronger locally convex topology on $X$ ).

The following theorem in essential part belongs to S. Kakutani and V. Klee [18]:

Theorem 0.15. $\mathbb{C}_{M}$ is a Brauner space if and only if the set $M$ is at most countable. In this (and only in this) case the topology of $\mathbb{C}_{M}$ coincides with the so called finite topology on $\mathbb{C}_{M}$ (a set $A$ is said to be closed in finite topology, if its intersection $A \cap F$ with any finite-dimensional subspace $F \subseteq \mathbb{C}_{M}$ is closed in $F$ ).

Duality between $\mathbb{C}^{M}$ and $\mathbb{C}_{M}$.

Theorem 0.16. The bilinear form

$$
\langle u, \alpha\rangle=\sum_{x \in M} u(x) \cdot \alpha_{x}, \quad u \in \mathbb{C}^{M}, \alpha \in \mathbb{C}_{M}
$$

turns $\mathbb{C}^{M}$ and $\mathbb{C}_{M}$ into a dual pair $\left\langle\mathbb{C}^{M}, \mathbb{C}_{M}\right\rangle$ of stereotype spaces:

(i) every point charge $\alpha \in \mathbb{C}_{M}$ generates a linear continuous functional $f$ on $\mathbb{C}^{M}$ by the formula

$$
f(u)=\langle u, \alpha\rangle, \quad u \in \mathbb{C}^{M},
$$

and the mapping $\alpha \mapsto f$ is an isomorphism of locally convex spaces

$$
\mathbb{C}_{M} \cong\left(\mathbb{C}^{M}\right)^{\star}
$$

(ii) on the contrary, every function $u \in \mathbb{C}^{M}$ generates a linear continuous functional $f$ on $\mathbb{C}_{M}$ by the formula

$$
f(\alpha)=\langle u, \alpha\rangle, \quad \alpha \in \mathbb{C}_{M},
$$

and the mapping $u \mapsto f$ is an isomorphism of locally convex spaces

$$
\mathbb{C}^{M} \cong\left(\mathbb{C}_{M}\right)^{\star}
$$

Bases in $\mathbb{C}^{M}$ and $\mathbb{C}_{M}$. A basis in a topological vector space $X$ over $\mathbb{C}$ is a family of vectors $\left\{e_{i} ; i \in I\right\}$ in $X$ such that every vector $x \in X$ can be uniquely represented as a sum of a converging series in $X$

$$
x=\sum_{i \in I} \lambda_{i} \cdot e_{i}
$$

with coefficients $\lambda_{i}=\lambda_{i}(x)$ continuously depending on $x \in X$. The summability of series (0.27) is understood in the sense of Bourbaki [6]: for each neighborhood of zero $U$ in $X$ there exists a finite set $J \subseteq I$ such that for any its finite superset $K, J \subseteq K \subseteq I$

$$
x-\sum_{i \in I} \lambda_{i} \cdot e_{i} \in U
$$

(the summability of series (0.27) does not mean that this series must have finite or countable number of nonzero terms).

Theorem 0.17. The characteristic functions $\left\{1_{x} ; x \in G\right\}$ of singletons $\{x\} \subseteq M$ :

$$
1_{x}(y)=\left\{\begin{array}{ll}
1, & x=y \\
0 & x \neq y
\end{array}, \quad y \in M\right.
$$

form a basis in the topological vector space $\mathbb{C}^{M}$ : every function $u \in \mathbb{C}^{M}$ is a sum of a series

$$
u=\sum_{x \in M} u(x) \cdot 1_{x}
$$

with coefficients $u(x) \in \mathbb{C}$ continuously depending on $u \in \mathbb{C}^{M}$. 
Theorem 0.18. The characteristic functions of singletons $\{x\} \subseteq M$, which as elements of $\mathbb{C}_{M}$ we denote by the Kronecker symbols $\left\{\delta^{x} ; x \in G\right\}$

$$
\delta^{x}(y)=\left\{\begin{array}{ll}
1, & x=y \\
0 & x \neq y
\end{array}, \quad y \in M\right.
$$

form a basis in the topological vector space $\mathbb{C}_{M}$ : every point charge $\alpha \in \mathbb{C}_{M}$ is a sum of a series

$$
\alpha=\sum_{x \in M} \alpha_{x} \cdot \delta^{x}
$$

with coefficients $\alpha_{x} \in \mathbb{C}$ continuously depending on $\alpha \in \mathbb{C}_{M}$.

Theorem 0.19. The bases $\left\{1_{x} ; x \in M\right\}$ (in $\left.\mathbb{C}^{M}\right)$ and $\left\{\delta^{x} ; x \in M\right\}$ (in $\mathbb{C}_{M}$ ) are dual:

$$
\left\langle 1_{x}, \delta^{y}\right\rangle= \begin{cases}1, & x=y \\ 0, & x \neq y\end{cases}
$$

Theorem 0.20. In the spaces $\mathbb{C}^{M}$ and $\mathbb{C}_{M}$ any two bases can be transformed into each other through some automorphism (i.e. a linear homeomorphism of the space into itself).

\section{$\S 1$ Stereotype Hopf algebras}

\section{(a) Tensor products and the structure of monoidal category on Ste}

If $X$ and $Y$ are stereotype spaces, then by $Y: X$ we denote a set of all linear continuous mappings $\varphi: X \rightarrow Y$ endowed with the topology of uniform convergence on totally bounded sets in $X$. The symbol $Y \oslash X$ means pseudosaturation of this space:

$$
Y \oslash X=(Y: X)^{\Delta}
$$

(the operation of pseudosaturation $\Delta$ means some special strengthening of the topology of the initial space - see [1, §1]). The space $Y \oslash X$ is always stereotype (if $X$ and $Y$ are stereotype).

In the category $\mathfrak{S t e}$ of stereotype spaces there are two natural tensor products:

- a projective tensor product is defined by the equality

$$
X \circledast Y=\left(X^{\star} \oslash Y\right)^{\star} ;
$$

the corresponding elementary tensor $x \circledast y \in X \circledast Y(x \in X, y \in Y)$ is defined by the formula

$$
x \circledast y(\varphi)=\varphi(y)(x), \quad \varphi \in X^{\star} \oslash Y ;
$$

- an injective tensor product is defined by the equality

$$
X \odot Y=X \oslash Y^{\star}
$$

the corresponding elementary tensor $x \odot y \in X \odot Y(x \in X, y \in Y)$ is defined by the formula

$$
x \odot y(f)=f(y) \cdot x, \quad f \in Y^{\star} .
$$

These two operations are connected with each other by two isomorphisms of functors:

$$
\mathrm{d}:(X \circledast Y)^{\star} \cong X^{\star} \odot Y^{\star}, \quad \mathrm{e}:(X \odot Y)^{\star} \cong X^{\star} \circledast Y^{\star}
$$


Theorem 1.1. The identities

$$
\begin{array}{ll}
\mathrm{a}_{X, Y, Z}^{\circledast}((x \circledast y) \circledast z)=x \circledast(y \circledast z) & x \in X, \quad y \in Y, \quad z \in Z \\
\mathrm{I}_{X}^{\circledast}(\lambda \circledast x)=\lambda \cdot x=\mathrm{r}_{X}^{\circledast}(x \circledast \lambda) & \lambda \in \mathbb{C}, \quad x \in X \\
\mathrm{c}_{X}^{\circledast}(x \circledast y)=y \circledast x & x \in X, \quad y \in Y
\end{array}
$$

correctly define natural isomorphisms of functors in the category $\mathfrak{S t e}$

$$
\begin{aligned}
& \mathrm{a}_{X, Y, Z}^{\circledast}:(X \circledast Y) \circledast Z \rightarrow X \circledast(Y \circledast Z) \\
& \mathrm{I}_{X}^{\circledast}: \mathbb{C} \circledast X \rightarrow X \\
& \mathrm{r}_{X}^{\circledast}: X \circledast \mathbb{C} \rightarrow X \\
& \mathrm{c}_{X, Y}^{\circledast}: X \circledast Y \rightarrow Y \circledast X
\end{aligned}
$$

These isomorphisms in their turn define a structure of symmetrical monoidal category on Ste [4, 23] with respect to the bifunctor $\circledast$.

Before formulating the next theorem let us agree to denote by $\mathrm{h}$ the mapping from $\mathbb{C}$ into $\mathbb{C}^{\star}$, which every number $\lambda \in \mathbb{C}$ turns into the linear functional on $\mathbb{C}$, acting as multiplication by $\lambda$ :

$$
\mathrm{h}: \mathbb{C} \rightarrow \mathbb{C}^{\star} \mid \mathrm{h}(\lambda)(\mu)=\lambda \cdot \mu \quad(\lambda, \mu \in \mathbb{C})
$$

Clearly, $\mathrm{h}: \mathbb{C} \rightarrow \mathbb{C}^{\star}$ is an isomorphism of (finite-dimensional) stereotype spaces.

Theorem 1.2. The formulas

$$
\begin{aligned}
& \mathrm{a}_{X, Y, Z}^{\odot}=\left(\mathrm{i}_{X} \odot\left(\mathrm{i}_{Y} \odot \mathrm{i}_{Z}\right) \circ 1_{X^{\star \star}} \odot \mathrm{d}_{Y^{\star}, Z^{\star}}^{-1} \circ \mathrm{d}_{X^{\star}, Y^{\star} \circledast Z^{\star}}^{-1} \circ\left(\mathrm{a}_{X^{\star}, Y^{\star}, Z^{\star}}^{\circledast}\right)^{\star} \circ\right. \\
& \left.\circ \mathrm{d}_{X^{\star} \circledast Y^{\star}, Z^{\star}} \circ \mathrm{d}_{X^{\star}, Y^{\star}} \odot 1_{Z^{\star \star}} \circ\left(\mathrm{i}_{X^{\star}}^{-1} \odot \mathrm{i}_{Y^{\star}}^{-1}\right) \odot \mathrm{i}_{Z^{\star}}^{-1}\right)^{\star} \\
& \mathrm{I}_{X}^{\odot}=\left(\mathrm{h}^{-1} \odot \mathrm{i}_{X}^{-1} \circ \mathrm{d}_{\mathbb{C}, X^{\star}} \circ\left(\mathrm{I}_{X^{\star}}^{\circledast}\right)^{\star} \circ \mathrm{i}_{X}\right)^{-1} \\
& \mathrm{r}_{X}^{\odot}=\left(\mathrm{i}_{X}^{-1} \odot \mathrm{h}^{-1} \circ \mathrm{d}_{X^{\star}, \mathbb{C}} \circ\left(\mathrm{r}_{X^{\star}}^{\circledast}\right)^{\star} \circ \mathrm{i}_{X}\right)^{-1} \\
& \mathrm{c}_{X, Y}^{\odot}=\left(\mathrm{i}_{X}^{-1} \odot \mathrm{i}_{Y}^{-1} \circ \mathrm{d}_{X^{\star}, Y^{\star}} \circ\left(\mathrm{c}_{X^{\star}, Y^{\star}}^{\circledast}\right)^{\star} \circ\left(\mathrm{d}_{Y^{\star}, X^{\star}}\right)^{-1} \circ \mathrm{i}_{Y} \odot \mathrm{i}_{X}\right)^{-1}
\end{aligned}
$$

define isomorphisms of functors in the category $\mathfrak{S t e}$

$$
\begin{aligned}
& \mathrm{a}_{X, Y, Z}^{\odot}:(X \odot Y) \odot Z \rightarrow X \odot(Y \odot Z) \\
& \mathrm{I}_{X}^{\odot}: \mathbb{C} \odot X \rightarrow X \\
& \mathrm{r}_{X}^{\odot}: X \odot \mathbb{C} \rightarrow X \\
& \mathrm{c}_{X, Y}^{\odot}: X \odot Y \rightarrow Y \odot X
\end{aligned}
$$

such that

$$
\begin{array}{ll}
\mathrm{a}_{X, Y, Z}^{\odot}((x \odot y) \odot z)=x \odot(y \odot z) & x \in X, \quad y \in Y, \quad z \in Z \\
\mathrm{I}_{X}^{\odot}(\lambda \odot x)=\lambda \cdot x=\mathrm{r}_{X}^{\odot}(x \odot \lambda) & \lambda \in \mathbb{C}, \quad x \in X \\
\mathrm{c}_{X}^{\odot}(x \odot y)=y \odot x & x \in X, \quad y \in Y
\end{array}
$$

These isomorphisms define a structure of symmetric monoidal category on Ste [4] with respect to the bifunctor $\odot$. 
The following fact was noted in [1, Theorem 7.9]: the identity

$$
\text { @ }_{X, Y}(x \circledast y)=x \odot y, \quad x \in X, \quad y \in Y
$$

defines a natural transformation of the bifunctor $\circledast$ into the bifunctor $\odot$

$$
@_{X, Y}: X \circledast Y \rightarrow X \odot Y,
$$

called Grothendieck transformation.

Theorem 1.3. If $X$ and $Y$ are Fréchet spaces (respectively, Brauner spaces), then

(i) the stereotype tensor products $X \circledast Y$ and $X \odot Y$ are also Fréchet spaces (respectively, Brauner spaces),

(ii) if at least one of the spaces $X$ and $Y$ is nuclear, then the Grothendieck mapping $@_{X, Y}: X \circledast Y \rightarrow$ $X \odot Y$ is an isomorphism of stereotype spaces

$$
X \circledast Y \stackrel{{ }_{X}}{\cong} X \odot Y
$$

(iii) if both of the spaces $X$ and $Y$ are nuclear, then the space $X \circledast Y \cong X \odot Y$ is also nuclear.

Proof. The fact that the spaces $X \circledast Y$ and $X \odot Y$ are Fréchet spaces (respectively, Brauner spaces), was noted in [1, Theorems 7.22, 7.23]). If $X$ or $Y$ is nuclear, then the isomorphism $X \circledast Y \cong X \odot Y$ is a corollary of the fact that for two Fréchet spaces, of which one possesses the classical approximation property, the tensor products $\circledast$ and $\odot$ coincide with the usual projective $\hat{\otimes}$ and injective $\check{\otimes}$ tensor products [1, Theorems 7.17, 7.21]. Finally, if both $X$ and $Y$ are nuclear, then in the case when both $X$ and $Y$ are Fréchet spaces, their tensor products $X \circledast Y \cong X \odot Y \cong X \hat{\otimes} Y$ are nuclear, since nuclearity is inherited by projective tensor product [25, 5.4.2]. If $X$ and $Y$ are Brauner spaces, then by what we have already proved, the Fréchet space $X^{\star} \odot Y^{\star}$ is nuclear, hence, by the Brauner theorem 0.10 , the space $X \circledast Y \cong\left(X^{\star} \odot Y^{\star}\right)^{\star}$ is also nuclear.

As a corollary, we have

Theorem 1.4. The categories $\mathfrak{N F r e}$ of nuclear Fréchet spaces and $\mathfrak{N B \mathfrak { r a }}$ of nuclear Brauner spaces are symmetrical monoidal categories with respect to bifunctors $\circledast$ and $\odot$ (which coincide on each of those categories).

\section{(b) Stereotype Hopf algebras}

Algebras, coalgebras and Hopf algebras in a symmetric monoidal category. Recall 23, that an algebra or a monoid in a symmetrical monoidal category $(\mathfrak{K}, \otimes, I, \mathrm{a}, \mathrm{r}, \mathrm{l}, \mathrm{l})$ is a triple $(A, \mu, \iota)$, where $A$ is an object in $\mathfrak{K}$, and $\mu: A \otimes A \rightarrow A$ (multiplication) and $\iota: I \rightarrow A$ (identity) are morphisms, satisfying the following axioms of associativity and identity:
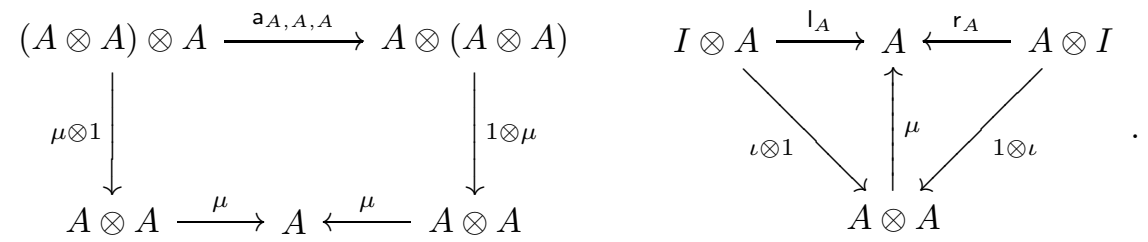

To any two monoids $\left(A, \mu_{A}, \iota_{A}\right)$ and $\left(B, \mu_{B}, \iota_{B}\right)$ one can assign a monoid $\left(A \otimes B, \mu_{A \otimes B}, \iota_{A \otimes B}\right)$ in which the structure morphisms are defined by formulas

$$
\mu_{A \otimes B}:=\mu_{A} \otimes \mu_{B} \circ \theta_{A, B, A, B}, \quad \iota_{A \otimes B}:=\iota_{A} \otimes \iota_{B} \circ \mathrm{I}_{I}^{-1}
$$




$$
(A \otimes B) \otimes(A \otimes B) \underset{\mu_{A \otimes B}->{ }_{A \otimes B}}{\theta_{A, B, B}}(A \otimes A) \otimes(B \otimes B)
$$

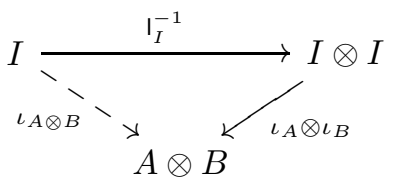

and $\theta$ is the isomorphism of functors

$$
((A, B, C, D) \mapsto(A \otimes B) \otimes(C \otimes D)) \stackrel{\theta}{\longmapsto}((A, B, C, D) \mapsto(A \otimes C) \otimes(B \otimes D))
$$

coming in as a combination of structure isomorphisms a, I, r, c, $\mathrm{a}^{-1}, \mathrm{I}^{-1}, \mathrm{r}^{-1}, \mathrm{c}^{-1}$ in the tensor category $\mathfrak{K}$ (by the coherence theorem [23] this morphism is unique in this class).

The notion of coalgebra or comonoid in a symmetric monoidal category $\mathfrak{K}$ is defined dually as an arbitrary triple $(A, \varkappa, \varepsilon)$, where $A$ is an object of $\mathfrak{K}$, and $\varkappa: A \rightarrow A \otimes A$ (comultiplication) and $\varepsilon: A \rightarrow I$ (counit) are morphisms satisfying the dual conditions of coassociativity and counit:
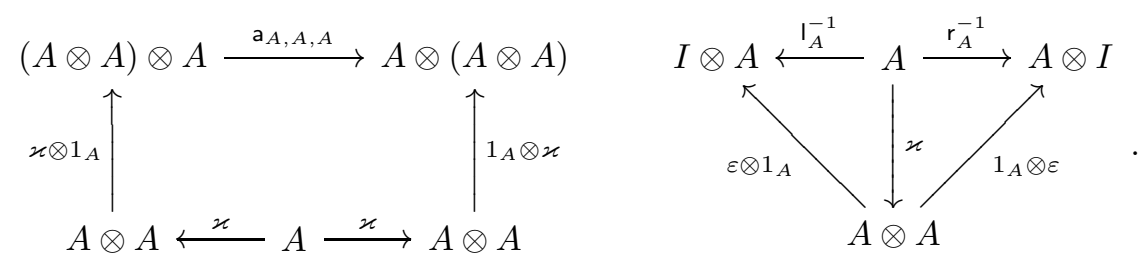

Like in the case of algebras, the tensor product $A \otimes B$ of two coalgebras $\left(A, \varkappa_{A}, \varepsilon_{A}\right)$ and $\left(B, \varkappa_{B}, \varepsilon_{B}\right)$ in $\mathfrak{K}$ possesses a natural structure of comonoid in the category $\mathfrak{K}$ with the structure morphisms

$$
\varkappa_{A \otimes B}:=\theta_{A, A, B, B} \circ \varkappa_{A} \otimes \varkappa_{B}, \quad \varepsilon_{A \otimes B}:=\lambda_{I} \circ \varepsilon_{A} \otimes \varepsilon_{B}
$$

Hopf algebra (another term - Hopf monoid) in the symmetrical monoidal category $\mathfrak{K}$ is a quintuple $(H, \mu, \iota, \varkappa, \varepsilon, \sigma)$, where $H$ is an object in $\mathfrak{K}$, and morphisms

$$
\begin{array}{lr}
\mu: H \otimes H \rightarrow H & \text { (multiplication), } \\
\iota: I \rightarrow H & \text { (unit), } \\
\varkappa: H \rightarrow H \otimes H & \text { (comultiplication), } \\
\varepsilon: H \rightarrow I & \text { (counit), } \\
\sigma: H \rightarrow H & \text { (antipode) }
\end{array}
$$

satisfy the following conditions:

1) the triple $(H, \mu, \iota)$ is an algebra in $\mathfrak{K}$,

2) the triple $(H, \varkappa, \varepsilon)$ is a coalgebra in $\mathfrak{K}$,

3) the following diagrams are commutative:

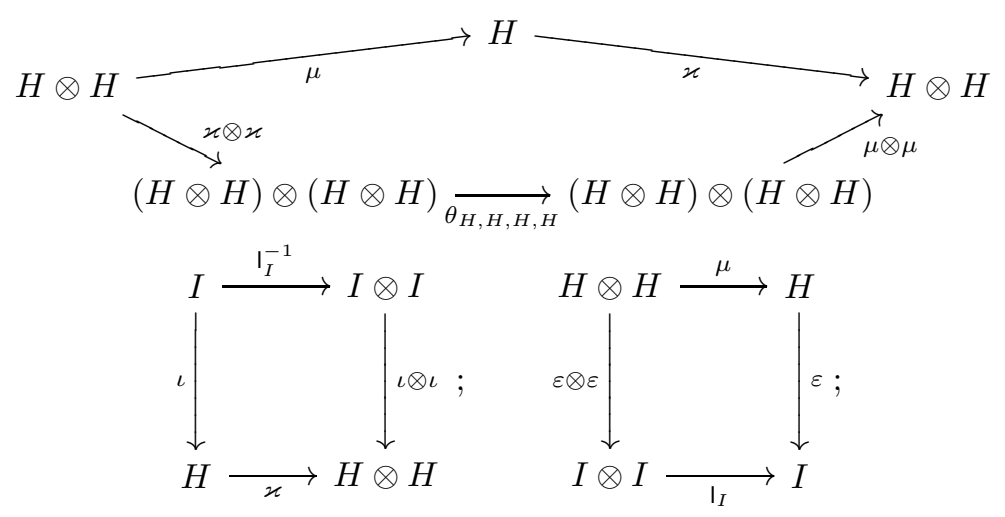




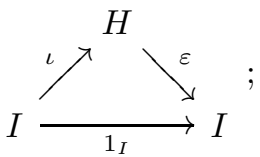

- they mean that morphisms $\varkappa: H \rightarrow H \otimes H$ and $\varepsilon: H \rightarrow I$ are homomorphisms of algebras, and morphisms $\mu: H \otimes H \rightarrow H$ and $\iota: I \rightarrow H$ are homomorphisms of coalgebras in the category $\mathfrak{K}$ (here again $\theta$ is the transformation (1.13));

4) the following diagram called antipode axiom is commutative:

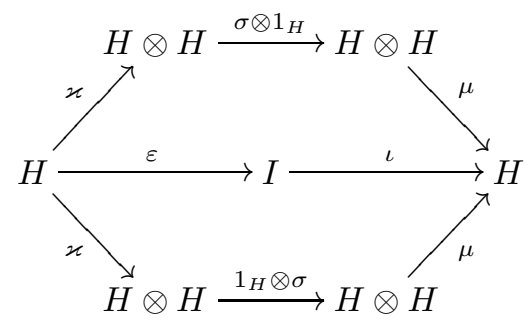

If only conditions 1)-3) are fulfilled, then the quadruple $(H, \mu, \iota, \varkappa, \varepsilon)$ is called a bialgebra in the category $\mathfrak{K}$.

Projective and injective stereotype algebras. In accordance with the general definition, a projective (respectively, injective) stereotype algebra is an algebra in the symmetric monoidal category of stereotype spaces $(\mathfrak{S t e}, \circledast)$ (respectively, $(\mathfrak{S t e}, \odot))$.

For the case of projective algebras this definition admits a simple reformulation [1, §10]:

Proposition 1.1. The structure of projective stereotype algebra on a stereotype space $A$ is equivalent to the structure of associative (unital) algebra on $A$, where the multiplication $(x, y) \mapsto x \cdot y$ satisfies the following two equivalent conditions of continuity:

(i) for every compact set $K$ in $A$ and for every neighborhood of zero $U$ in $A$ there exists a neighborhood of zero $V$ in $A$ such that

$$
K \cdot V \subseteq U \quad \& \quad V \cdot K \subseteq U
$$

(ii) for every compact set $K$ in $A$ and for any net $a_{i}$ in $A$, tending to zero, $a_{i} \underset{i \rightarrow \infty}{\longrightarrow} 0$, the nets $x \cdot a_{i}$ and $a_{i} \cdot x$ tend to zero in $A$ uniformly in $x \in K$.

Example 1.1. Banach algebras and Fréchet algebras are examples of projective stereotype algebras. Another example is the stereotype algebra $\mathcal{L}(X)=X \oslash X$ of operators on an arbitrary stereotype space $X$.

Example 1.2. The standard functional algebras $\mathcal{C}(M), \mathcal{E}(M), \mathcal{O}(M), \mathcal{R}(M)$ of continuous, smooth, holomorphic functions and polynomials are examples of injective stereotype algebras (see details in [1, $\S 10])$.

Stereotype Hopf algebras. Again, following the general definition, we call

- a projective stereotype Hopf algebra a Hopf algebra in the symmetric monoidal category of stereotype spaces with the projective tensor product $(\mathfrak{S t e}, \circledast)$;

- an injective stereotype Hopf algebra a Hopf algebra in the symmetric monoidal category of stereotype spaces with the injective tensor product $(\mathfrak{S t e}, \odot)$; 
- a nuclear Hopf-Fréchet algebra a Hopf algebra in the symmetric monoidal category of nuclear Fréchet spaces $\mathfrak{N F r e}$

- a nuclear Hopf-Brauner algebra a Hopf algebra in the symmetric monoidal category of nuclear Brauner spaces $\mathfrak{N B r a}$.

Suppose in addition that a stereotype space $H$ is such that the Grothendieck transformation for the pair $(H ; H)$, for the triple $(H ; H ; H)$, and for the quadruple $(H ; H ; H ; H)$ give isomorphisms of stereotype spaces:

$$
\begin{aligned}
& @_{H, H}: H \circledast H \cong H \odot H, \\
& @_{H, H, H}: H \circledast H \circledast H \cong H \odot H \odot H \\
& @_{H, H, H, H}: H \circledast H \circledast H \circledast H \cong H \odot H \odot H \odot H
\end{aligned}
$$

(this is always the case if $H$ is a nuclear Fréchet space or a nuclear Brauner space). Then, obviously, every structure of projective stereotype Hopf algebra on $H$ is equivalent to some structure of injective stereotype Hopf algebra on $H$ and vice versa: the structure elements of Hopf algebra in $(\mathfrak{S t e}, \circledast)$ and $(\mathfrak{S t e}, \odot)$ (we differ them by indices $\circledast$ and $\odot$ ) either coincide

$$
\iota_{\circledast}=\iota_{\odot}, \quad \varepsilon_{\circledast}=\varepsilon_{\odot}, \quad \sigma_{\circledast}=\sigma_{\odot}
$$

or are connected by diagrams
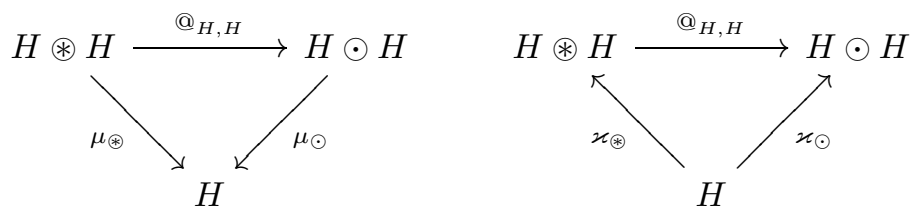

We call those (projective and at the same time injective) Hopf algebras rigid stereotype Hopf algebras.

Example 1.3. Clearly, every nuclear Hopf-Fréchet algebra and every nuclear Hopf-Brauner algebra are rigid stereotype Hopf algebras.

\section{Duality for stereotype Hopf algebras.}

Theorem 1.5 (on duality for stereotype Hopf algebras). A structure of injective (projective, rigid) Hopf algebra on a stereotype space $H$ automatically defines a structure of projective (injective, rigid) Hopf algebra on the dual stereotype space $H^{\star}$ - the structure elements of Hopf algebra on $H^{\star}$ are defined as the dual morphisms for the structure elements of Hopf algebra on $\mathrm{H}$ :

$$
\begin{array}{ll}
\mu_{H^{\star}}=\left(\varkappa_{H}\right)^{\star}, & \iota_{H^{\star}}=\left(\varepsilon_{H}\right)^{\star}, \\
\varkappa_{H^{\star}}=\left(\mu_{H}\right)^{\star}, & \varepsilon_{H^{\star}}=\left(\iota_{H}\right)^{\star}, \\
\sigma_{H^{\star}}=\left(\sigma_{H}\right)^{\star} . &
\end{array}
$$

Example 1.4. Every Hopf algebra in usual sense $H$ (i.e. a Hopf algebra in the category of vector spaces with the usual algebraic tensor product $\otimes$ ) becomes a rigid Hopf algebra, being endowed with the strongest locally convex topology. The dual space $H^{\star}$ with respect to this topology is a space of minimal type (in the sense of Theorem 0.12) and a rigid Hopf algebra, as well as $H$. This, by the way, illustrates one of the advantages of stereotype theory: here we do not need to narrow the space of linear functionals to make a Hopf algebra from them, like it is usually done (see e.g. [9, 1.5] or [8, 4.1.D]) - the space $H^{\star}$, being a space of all linear functionals (automatically continuous, by the choice of the topology in $H$ ), is a "true" Hopf algebra, but to see this we have to take one of the stereotype tensor products $\circledast$ or $\odot$ instead of the algebraic tensor product $\otimes$. 
Dual pairs. Let $H$ be an injective, and $M$ a projective stereotype Hopf algebras. Let us say that $H$ and $M$ form a dual pair of Hopf algebras, if there is a non-degenerate continuous (in the sense of [1, Section 5.6]) bilinear form $\langle\cdot, \cdot\rangle: H \times M \rightarrow \mathbb{C}$, which turns algebraic operations in $H$ into dual operations in $M$ :

$$
\begin{aligned}
& \left\langle\mu_{H}(U), \alpha\right\rangle=\left\langle U, \varkappa_{M}(\alpha)\right\rangle, \\
& \left\langle 1_{H}, \alpha\right\rangle=\varepsilon_{A}(\alpha), \\
& \left\langle\varkappa_{H}(u), A\right\rangle=\left\langle u, \mu_{M}(A)\right\rangle, \\
& \varepsilon_{H}(u)=\left\langle u, 1_{M}\right\rangle \text {, } \\
& \left\langle\sigma_{H}(u), \alpha\right\rangle=\left\langle u, \sigma_{M}(\alpha)\right\rangle
\end{aligned}
$$

( $u \in H, U \in H \odot H, \alpha \in M, A \in M \circledast M)$. If $\langle H, M\rangle$ is a dual pair of stereotype Hopf algebras, where $H$ is an injective, $M$ a projective Hopf algebra, then on default we shall denote by dot · the multiplication in $H$, by snowflake $*$ the multiplication in $M$ :

$$
\mu_{H}(u \odot v)=u \cdot v, \quad \mu_{M}(\alpha \circledast \beta)=\alpha * \beta
$$

Example 1.5. Certainly, the pair $\left\langle H, H^{\star}\right\rangle$, where $H$ is an injective stereptype Hopf algebra, and $\langle\cdot, \cdot\rangle$ the canonical bilinear form,

$$
\langle a, \alpha\rangle:=\alpha(a), \quad a \in H, \alpha \in H^{\star}
$$

is an example of dual pair of Hopf algebras. Below we use the notation $\langle\cdot, \cdot\rangle$ for this form without supplementary explanations.

\section{(c) Key example: Hopf algebras $\mathbb{C}^{G}$ and $\mathbb{C}_{G}$}

Recall that in $\S \mathbb{( h )}$ we defined the spaces $\mathbb{C}^{M}$ and $\mathbb{C}_{M}$ of functions and of point charges on a set $M$. If $M$ is a group, then the spaces $\mathbb{C}^{M}$ and $\mathbb{C}_{M}$ naturally turn into a dual pair of Hopf algebras.

Algebra $\mathbb{C}^{G}$ of functions on $G$. Let $G$ be an arbitrary group (not necessarily finite) and let $\mathbb{C}^{G}$ be the space of all (complex-valued) functions on $G$, defined in $\S$ q(h),

$$
u \in \mathbb{C}^{G} \Longleftrightarrow u: G \rightarrow \mathbb{C}
$$

with the topology of pointwise convergence (generated by seminorms $(0.23)$ ). We endow $\mathbb{C}^{G}$ with the supplementary structure of algebra with the pointwise algebraic operations:

$$
(u \cdot v)(x)=u(x) \cdot v(x), \quad 1_{\mathbb{C}^{G}}(x)=1 \quad(x \in G) .
$$

Recall that by formula (0.28) above we defined the characteristic functions $1_{x}$ of singletons in $G$. The multiplication in $\mathbb{C}^{G}$ can be written in the decomposition by the basis $\left\{1_{x} ; x \in G\right\}$ by formula

$$
u \cdot v=\left(\sum_{x \in G} u(x) \cdot 1_{x}\right) \cdot\left(\sum_{x \in G} v(x) \cdot 1_{x}\right)=\sum_{x \in G} u(x) \cdot v(x) \cdot 1_{x}
$$

and on the elements of this basis looks as follows:

$$
1_{x} \cdot 1_{y}= \begin{cases}1_{x}, & x=y \\ 0 & x \neq y\end{cases}
$$

The algebra $\mathbb{C}_{G}$ of point charges on $G$. Again let $G$ be an arbitrary group and $\mathbb{C}_{G}$ the space of point charges on $G$, defined in $\S$ Q(h)

$$
\alpha \in \mathbb{C}_{G} \Longleftrightarrow \alpha=\left\{\alpha_{x} ; x \in G\right\}, \quad \alpha_{x} \in \mathbb{C}, \quad \operatorname{card}\left\{x \in G: \alpha_{x} \neq 0\right\}<\infty
$$


We endow $\mathbb{C}_{G}$ with the topology generated by seminorms 0.25 (by theorem 0.14 this is equivalent to the strongest locally convex topology on $\mathbb{C}_{G}$ ). Besides this $\mathbb{C}_{G}$ is endowed with the structure of algebra under the multiplication

$$
(\alpha * \beta)_{y}=\sum_{x \in G} \alpha_{x} \cdot \beta_{x^{-1} \cdot y}
$$

By formula (0.30) we defined the characteristic function of singletons in $G$, which, being considered as elements in $\mathbb{C}_{G}$ were denoted by the symbols of delta-functionals: $\delta^{x}$. The unit in $\mathbb{C}_{G}$ is the characteristic function $\delta^{e}$ supported in the unit $e$ of the group $G$ :

$$
\delta_{x}^{e}= \begin{cases}1, & x=e \\ 0 & x \neq e\end{cases}
$$

The multiplication in the algebra $\mathbb{C}_{G}$ is written in the decomposition by elements of the basis $\left\{\delta^{x} ; x \in G\right\}$ by formula

$$
\alpha * \beta=\left(\sum_{x \in G} \alpha_{x} \cdot \delta^{x}\right) *\left(\sum_{y \in G} \beta_{y} \cdot \delta^{y}\right)=\sum_{x, y \in G} \alpha_{x} \cdot \beta_{y} \cdot \delta^{x \cdot y}=\sum_{z \in G}\left(\sum_{x \in G} \alpha_{x} \cdot \beta_{x^{-1} \cdot z}\right) \cdot \delta^{z}
$$

and on the elements of this basis looks as follows:

$$
\delta^{x} * \delta^{y}=\delta^{x \cdot y}
$$

$\mathbb{C}^{G}$ and $\mathbb{C}_{G}$ as stereotype Hopf algebras. For two arbitrary sets $S$ and $T$ and for two functions $u: S \rightarrow \mathbb{C}$ and $v: T \rightarrow \mathbb{C}$ let the symbol $u \boxminus v$ denote the function on the Cartesian product $S \times T$ defined by the identity

$$
(u \boxminus v)(s, t):=u(s) \cdot v(t), \quad s \in S, \quad t \in T
$$

The definition of the Hopf algebra in the infinite-dimensional algebras $\mathbb{C}^{G}$ and $\mathbb{C}_{G}$ is based on the following observation:

Theorem 1.6. The formula

$$
\rho_{S, T}(u \boxminus v)=u \odot v
$$

defines an isomorphism of topological vector spaces

$$
\rho_{S, T}: \mathbb{C}^{S \times T} \cong \mathbb{C}^{S} \odot \mathbb{C}^{T}
$$

This isomorphism is an isomorphism of functors,

$$
\left((S ; T) \mapsto \mathbb{C}^{S \times T}\right) \stackrel{\rho_{S, T}}{\longmapsto}\left((S ; T) \mapsto \mathbb{C}^{S} \odot \mathbb{C}^{T}\right)
$$

since for any mappings $\pi: S \rightarrow S^{\prime}$ and $\sigma: T \rightarrow T^{\prime}$ the following diagram is commutative:

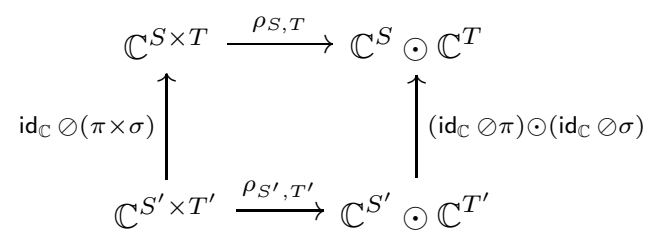

- here the mappings $\mathrm{id}_{\mathbb{C}} \oslash$ ? are defined by formula

$$
\operatorname{id}_{\mathbb{C}} \oslash \pi: \mathbb{C}^{S^{\prime}} \rightarrow \mathbb{C}^{S}, \quad \operatorname{id}_{\mathbb{C}} \oslash \pi(v)=v \circ \pi
$$


This theorem allows to define the structure elements of Hopf algebra on $\mathbb{C}^{G}$ with respect to $\odot:$ the mapping $\mu: \mathbb{C}^{G} \odot \mathbb{C}^{G} \rightarrow \mathbb{C}^{G}$ is initially defined on the set of functions on the Cartesian product $G \times G$,

$$
\widetilde{\mu}: \mathbb{C}^{G \times G} \rightarrow \mathbb{C}^{G} \mid \widetilde{\mu}(v)(t)=v(t, t)
$$

and then is extended to tensor square by the isomorphism $\rho_{G, G}$ :

$$
\mu=\widetilde{\mu} \circ \rho_{G, G} .
$$

Similarly the comultiplication $\varkappa: \mathbb{C}^{G} \rightarrow \mathbb{C}^{G} \odot \mathbb{C}^{G}$ is initially defined with the values in the space of functions on the Cartesian square

$$
\widetilde{\varkappa}: \mathbb{C}^{G} \rightarrow \mathbb{C}^{G \times G} \mid \tilde{\varkappa}(u)(s, t)=u(s \cdot t)
$$

and after that is extended to tensor square by the isomorphism $\rho_{G, G}$ :

$$
\varkappa=\rho_{G, G} \circ \tilde{\varkappa} \text {. }
$$

The other structure elements of Hopf algebra on $\mathbb{C}^{G}$ are obvious:

$$
\begin{array}{rll}
\text { unit: } & \iota: \mathbb{C} \rightarrow \mathbb{C}^{G}, & \iota(\lambda)(t)=\lambda \\
\text { counit: } & \varepsilon: \mathbb{C}^{G} \rightarrow \mathbb{C}, & \varepsilon(u)=u\left(1_{G}\right) \\
\text { antipode: } & \sigma: \mathbb{C}^{G} \rightarrow \mathbb{C}^{G}, & \sigma(u)(t)=u\left(t^{-1}\right)
\end{array}
$$

The following picture illustrates these definitions:

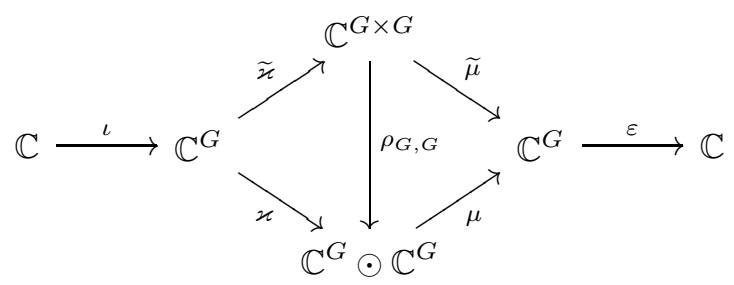

On the space $\mathbb{C}_{G}$ of point charges on $G$ the structure of Hopf algebra with respect to tensor product $\circledast$ is defined dually by Theorem [1.5] as on the dual space to $\mathbb{C}^{G}$ in the sense of bilinear form (0.26). The following theorem shows that these definitions indeed define a structure of Hopf algebra on $\mathbb{C}^{G}$ and $\mathbb{C}_{G}$ :

Theorem 1.7. For any group $G$

- the space $\mathbb{C}^{G}$ of functions on $G$ is an injective (and moreover, a rigid) stereotype Hopf algebra; if in addition $G$ is countable, then $\mathbb{C}^{G}$ is a nuclear Hopf-Fréchet algebra;

- the space $\mathbb{C}_{G}$ of point charges on $G$ is a projective (and moreover, a rigid) stereotype Hopf algebra; if in addition $G$ is countable, then $\mathbb{C}_{G}$ is a nuclear Hopf-Brauner algebra.

The Hopf algebras $\mathbb{C}^{G}$ and $\mathbb{C}_{G}$ form a dual pair with respect to bilinear form (0.26), and the algebraic operations on them act on bases $\left\{1_{x}\right\}$ and $\left\{\delta^{x}\right\}$ by formulas:

$$
\begin{aligned}
& \mathbb{C}^{G}: \quad 1_{x} \cdot 1_{y}=\left\{\begin{array}{ll}
1_{x}, & x=y \\
0, & x \neq y
\end{array} \quad 1_{\mathbb{C} G}=\sum_{x \in G} 1_{x} \quad \sigma\left(1_{x}\right)=1_{x^{-1}}\right. \\
& \varkappa\left(1_{x}\right)=\sum_{y \in G} 1_{y} \odot 1_{x \cdot y^{-1}} \quad \varepsilon\left(1_{x}\right)= \begin{cases}1, & x=e \\
0, & x \neq e\end{cases} \\
& \begin{array}{llll}
\mathbb{C}_{G}: & \delta^{x} * \delta^{y}=\delta^{x \cdot y} & 1_{\mathbb{C}_{G}}=\delta^{e} & \sigma\left(\delta^{x}\right)=\delta^{x^{-1}} \\
& \varkappa\left(\delta^{x}\right)=\delta^{x} \circledast \delta^{x} & \varepsilon\left(\delta^{x}\right)=1 &
\end{array}
\end{aligned}
$$


Proof. Formulas (1.27)-(1.30) are verified by direct calculation. The rigidity follows from [1, (7.37)]:

$$
\mathbb{C}^{I} \circledast \mathbb{C}^{J} \cong \mathbb{C}^{I \times J} \cong \mathbb{C}^{I} \odot \mathbb{C}^{J}, \quad \mathbb{C}_{I} \circledast \mathbb{C}_{J} \cong \mathbb{C}_{I \times J} \cong \mathbb{C}_{I} \odot \mathbb{C}_{J} .
$$

The structure of Hopf algebra on $\mathbb{C}_{G}$ is generated by the structure of Hopf algebra on $\mathbb{C}^{G}$ due to Theorem 1.5. Thus, we need only to prove that $\mathbb{C}^{G}$ is a Hopf algebra with respect to $\odot$.

1. Let us check diagram (1.15). Replace everywhere $H$ by $\mathbb{C}^{G}$ and $\otimes$ by $\odot$, and after that let us overbuild this diagram to the following prism:

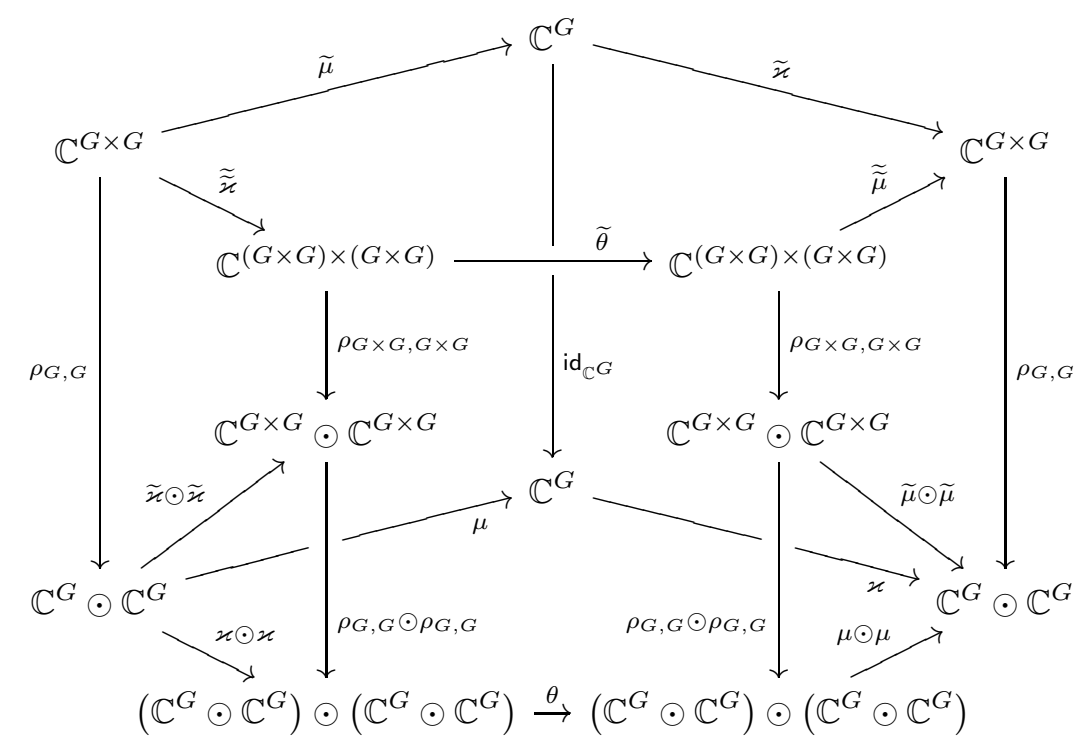

Here $\theta=\theta_{\mathbb{C}^{G}, \mathbb{C}^{G}, \mathbb{C}^{G}, \mathbb{C}^{G}}$ is the isomorphism of functors from (1.13), and the other morphisms are defined as follows:

$$
\widetilde{\theta} w(a, b, c, d)=w(a, c, b, d), \quad \widetilde{\widetilde{\varkappa}} v(a, b, c, d)=v(a \cdot b, c \cdot d), \quad \widetilde{\widetilde{\mu}} v(a, b)=v(a, a, b, b)
$$

To prove that the base of the prism is commutative, it is sufficient to verify that all the other faces are commutative. The remote lateral faces
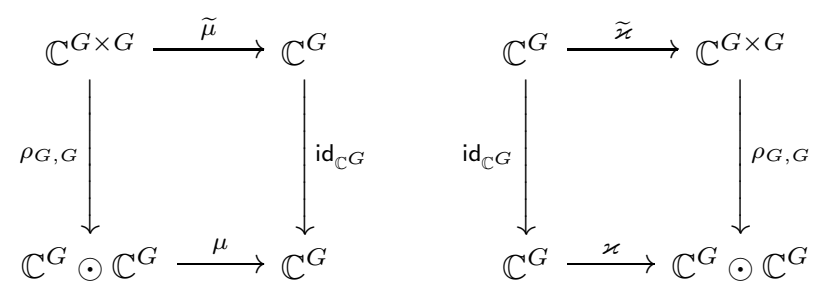

- are just distorted triangles from diagram (1.26).

In the left nearby face

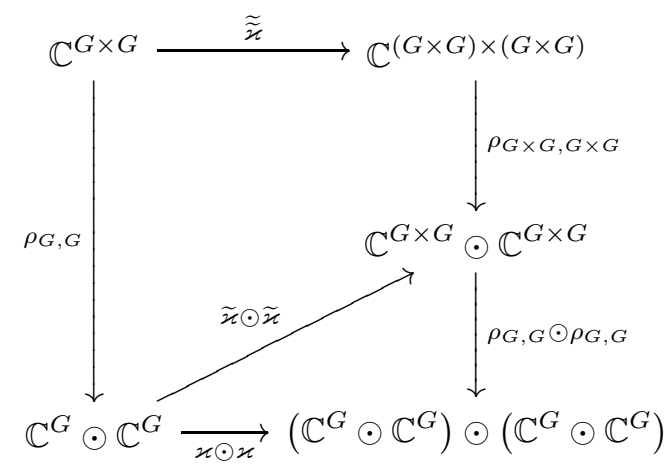


- the lower triangle is just left triangle in (1.26) multiplied by itself via the operation $\odot$, and the commutativity of the inner quadrangle is verified by the substituting the function $u \boxminus v \in \mathbb{C}^{G \times G}, u, v \in \mathbb{C}^{G}$, as an argument: if we move down, and then right and up, we turn this function into $\tilde{\varkappa}(u) \odot \tilde{\varkappa}(v)$,

$$
\begin{array}{ll} 
& u \odot v \in \mathbb{C}^{G \times G} \\
\mapsto \quad & u \odot v \in \mathbb{C}^{G} \odot \mathbb{C}^{G} \\
\mapsto \quad & (\widetilde{\varkappa} \odot \tilde{\varkappa})(u \odot v)=\tilde{\varkappa}(u) \odot \tilde{\varkappa}(v) \in \mathbb{C}^{G \times G} \odot \mathbb{C}^{G \times G}
\end{array}
$$

- and if we move right and then down, we obtain the same result:

$$
\begin{aligned}
& u \boxminus v \in \mathbb{C}^{G \times G} \\
& \mapsto \quad \tilde{\widetilde{\varkappa}}(u \boxminus v)=\tilde{\varkappa}(u) \boxminus \tilde{\varkappa}(v) \in \mathbb{C}^{(G \times G) \times(G \times G)}: \\
& \widetilde{\widetilde{\varkappa}}(u \boxminus v)(a, b, c, d)=(u \boxminus v)(a \cdot b, c \cdot d)=u(a \cdot b) \cdot v(c \cdot d)=\tilde{\varkappa}(u)(a, b) \cdot \tilde{\varkappa}(v)(c, d)= \\
& =(\tilde{\varkappa}(u) \square \tilde{\varkappa}(v))(a, b, c, d) \\
& \left.\mapsto \quad \rho_{G \times G, G \times G}(\tilde{\varkappa}(u) \square \tilde{\varkappa}(v))=(1.25)=\tilde{\varkappa}(u)\right) \odot \tilde{\varkappa}(v) \in \mathbb{C}^{G \times G} \odot \mathbb{C}^{G \times G} .
\end{aligned}
$$

The commutativity of the central nearby face:

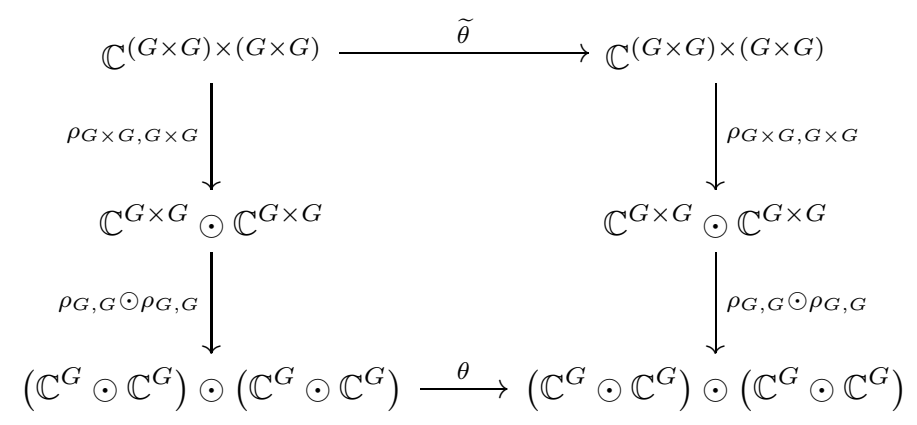

- is verified by substituting a function $(u \boxminus v) \boxminus(p \boxminus q) \in \mathbb{C}^{G \times G}$ as an argument, its motion due to (1.25) will be as follows:

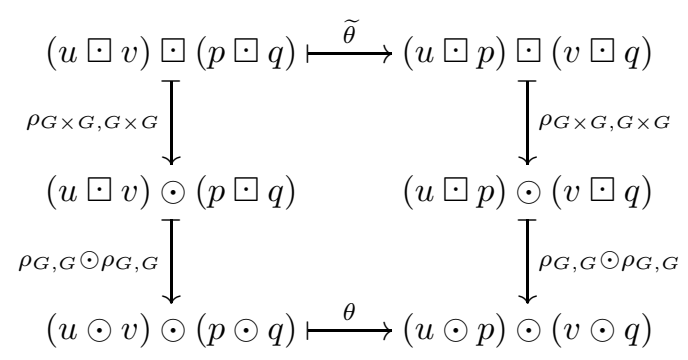

In the right nearby face

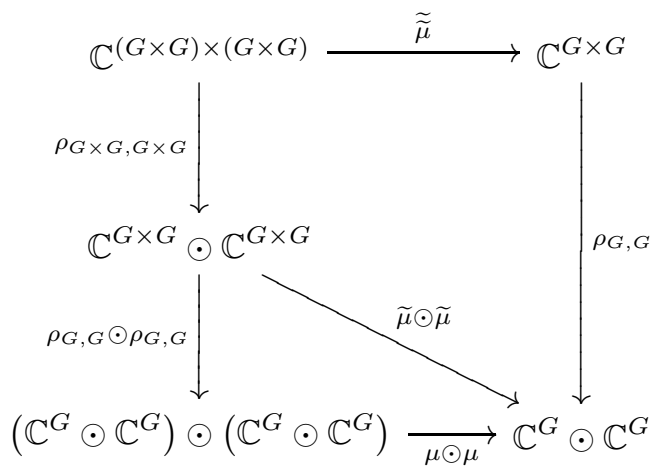


- the lower triangle is just the right triangle in (1.26) multiplied by itself via the operation $\odot$, and the commutativity of the inner quadrangle is verified by taking as an argument the function $u \boxminus v \in$ $\mathbb{C}^{(G \times G) \times(G \times G)}, u, v \in \mathbb{C}^{G \times G}$ : if we move down, and then right and down, we obtain

$$
\begin{array}{ll} 
& u \boxminus v \in \mathbb{C}^{(G \times G) \times(G \times G)} \\
\mapsto \quad & \rho_{G \times G, G \times G}(u \odot v)=u \odot v \in \mathbb{C}^{G \times G} \odot \mathbb{C}^{G \times G} \\
\mapsto \quad & (\widetilde{\mu} \odot \widetilde{\mu})(u \odot v)=\widetilde{\mu}(u) \odot \widetilde{\mu}(v) \in \mathbb{C}^{G} \odot \mathbb{C}^{G}
\end{array}
$$

- and if we move right and then down, we obtain the same result:

$$
\begin{array}{ll} 
& u \boxminus v \in \mathbb{C}^{(G \times G) \times(G \times G)} \\
\mapsto \quad & \widetilde{\widetilde{\mu}}(u \boxminus v)=\widetilde{\mu}(u) \boxminus \widetilde{\mu}(v) \in \mathbb{C}^{G \times G}: \\
& \widetilde{\widetilde{\mu}}(u \boxminus v)(a, b)=(u \boxminus v)(a, a, b, b)=u(a, a) \cdot v(b \cdot b)=\widetilde{\mu}(u)(a) \cdot \widetilde{\mu}(v)(b)=(\widetilde{\mu}(u) \boxminus \widetilde{\mu}(v))(a, b) \\
\left.\mapsto \quad \rho_{G, G}(\widetilde{\mu}(u) \boxminus \widetilde{\mu}(v))=(1.25)=\widetilde{\mu}(u)\right) \odot \widetilde{\mu}(v) \in \mathbb{C}^{G} \odot \mathbb{C}^{G} .
\end{array}
$$

It remains to check the commutativity of the upper base of the prism.

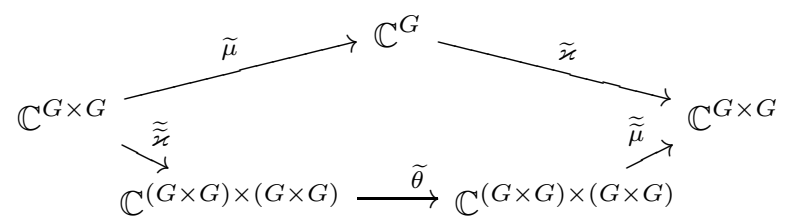

A function $v \in \mathbb{C}^{G \times G}$, being moved through the upper two edges of the pentagon, undergoes the following transmutations:

$$
\begin{array}{ll} 
& v \in \mathbb{C}^{G \times G} \\
\mapsto \quad \tilde{\mu} v \in \mathbb{C}^{G}, \quad \tilde{\mu} v(a)=v(a, a) \\
\mapsto \quad \tilde{\varkappa}(\widetilde{\mu} v) \in \mathbb{C}^{G \times G}, \quad \tilde{\varkappa}(\widetilde{\mu} v)(a, b)=\widetilde{\mu} v(a \cdot b)=v(a \cdot b, a \cdot b)
\end{array}
$$

- and the result is the same as if we move it through the three lower edges:

$$
\begin{array}{ll} 
& v \in \mathbb{C}^{G \times G} \\
\mapsto \quad & \widetilde{\widetilde{\varkappa}} v \in \mathbb{C}^{(G \times G) \times(G \times G)}, \quad \widetilde{\widetilde{\varkappa}} v(a, b, c, d)=v(a \cdot b, c \cdot d) \\
\mapsto \quad & \tilde{\theta}(\widetilde{\widetilde{\varkappa}} v) \in \mathbb{C}^{(G \times G) \times(G \times G)}, \quad \tilde{\theta}(\widetilde{\widetilde{\varkappa}} v)(a, b, c, d)=\widetilde{\varkappa} v(a, c, b, d)=v(a \cdot c, b \cdot d) \\
\mapsto \quad & \widetilde{\widetilde{\mu}}(\widetilde{\theta}(\widetilde{\widetilde{\varkappa}} v)) \in \mathbb{C}^{G \times G}, \quad \widetilde{\widetilde{\mu}}(\widetilde{\theta}(\widetilde{\tilde{\varkappa}} v))(a, b)=\widetilde{\theta}(\widetilde{\widetilde{\varkappa}} v)(a, a, b, b)=\widetilde{\widetilde{\varkappa}} v(a, b, a, b)=v(a \cdot b, a \cdot b)
\end{array}
$$

2. After that we verify diagrams (1.16), which for the algebra $\mathbb{C}^{G}$ come to the following form:
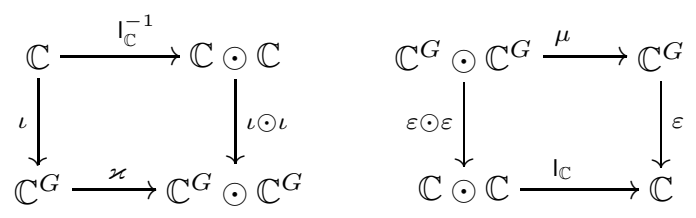

Let us input an arbitrary number $\zeta \in \mathbb{C}$ as a argument into the first diagram, and an elementary tensor $u \odot v \in \mathbb{C}^{G} \odot \mathbb{C}^{G}$ into the second diagram, and apply identities (1.8):
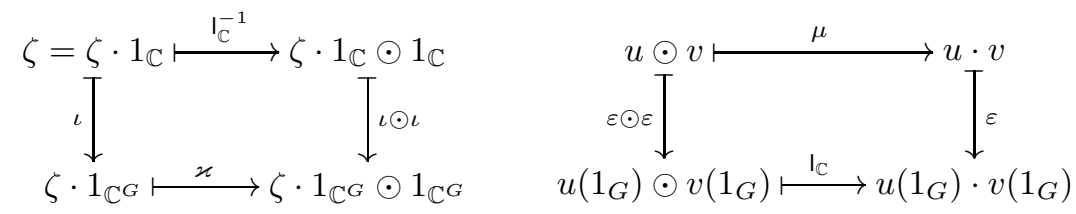
3. It remains to verify diagram (1.18), which for $\mathbb{C}^{G}$ comes to the form:

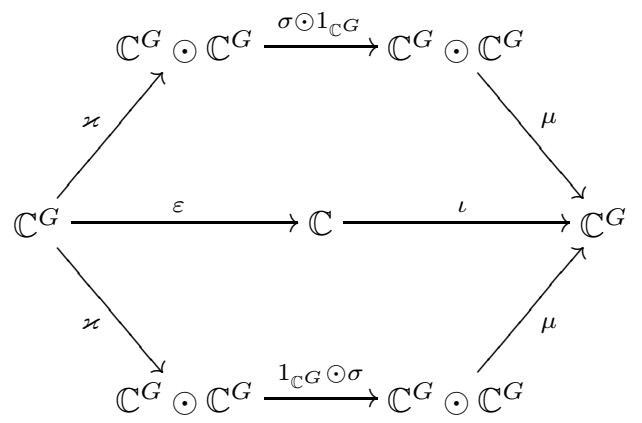

We overbuild it to the diagram

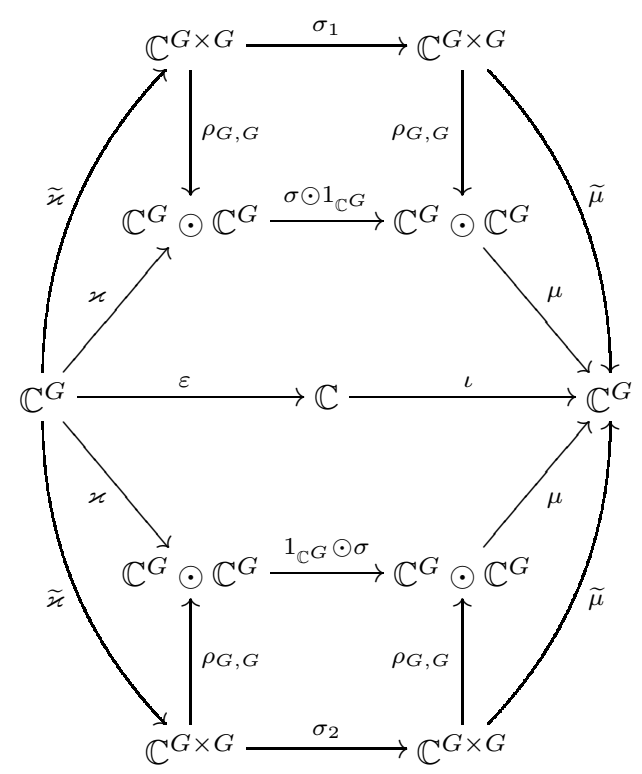

where the mappings $\sigma_{1}$ and $\sigma_{2}$ are defined by the identities

$$
\sigma_{1}(v)(s, t):=v\left(s^{-1}, t\right), \quad \sigma_{2}(v)(s, t):=v\left(s, t^{-1}\right)
$$

Obviously, all the triangles and quadrangles siding with the borders of this picture, are commutative here. Hence, to prove the commutativity of the two inner pentagons (i.e. the commutativity of (1.31)) it is sufficient to check the commutativity of the diagram arising after throwing out the vertexes $\mathbb{C}^{G} \odot \mathbb{C}^{G}$ :

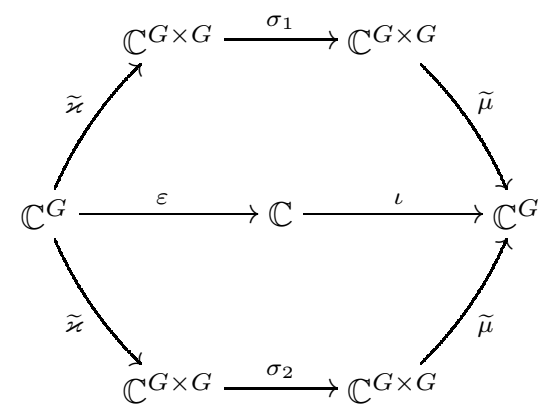

This is done by the direct calculation: for any function $u \in \mathbb{C}^{G}$ its final image after motion through the diagram is the function $u\left(1_{G}\right) \cdot 1_{\mathbb{C}^{G}} \in \mathbb{C}^{G}$. Indeed, 
- if we move through the upper arrows, we obtain the following:

$$
\begin{array}{ll} 
& u \in \mathbb{C}^{G} \\
\mapsto & \tilde{\varkappa}(u) \in \mathbb{C}^{G \times G}, \quad \tilde{\varkappa}(u)(s, t)=u(s \cdot t) \\
\mapsto & \sigma_{1}(\widetilde{\varkappa}(u)) \in \mathbb{C}^{G \times G}, \quad \sigma_{1}(\tilde{\varkappa}(u))(s, t)=\tilde{\varkappa}(u)\left(s^{-1}, t\right)=u\left(s^{-1} \cdot t\right) \\
\mapsto & \widetilde{\mu}\left(\sigma_{1}(\tilde{\varkappa}(u))\right) \in \mathbb{C}^{G}, \quad \tilde{\mu}\left(\sigma_{1}(\tilde{\varkappa}(u))\right)(s)=\sigma_{1}(\tilde{\varkappa}(u))(s, s)=\tilde{\varkappa}(u)\left(s^{-1}, s\right)=u\left(s^{-1} \cdot s\right)=u\left(1_{G}\right)
\end{array}
$$

- if we move aflat through the center of the pentagon, we obtain the same:

$$
\begin{aligned}
& u \in \mathbb{C}^{G} \\
& \mapsto \quad \varepsilon(u) \in \mathbb{C}, \quad \varepsilon(u)=u\left(1_{G}\right) \cdot 1_{\mathbb{C}} \\
& \mapsto \quad \iota(\varepsilon(u)) \in \mathbb{C}^{G}, \quad \iota(\varepsilon(u))=u\left(1_{G}\right) \cdot 1_{\mathbb{C}^{G}}
\end{aligned}
$$

- and if we move through the lower arrows, we come to the same result:

$$
\begin{array}{lll} 
& u \in \mathbb{C}^{G} \\
\mapsto & \tilde{\varkappa}(u) \in \mathbb{C}^{G \times G}, \quad \tilde{\varkappa}(u)(s, t)=u(s \cdot t) \\
\mapsto & \sigma_{2}(\widetilde{\varkappa}(u)) \in \mathbb{C}^{G \times G}, \quad \sigma_{2}(\widetilde{\varkappa}(u))(s, t)=\widetilde{\varkappa}(u)\left(s, t^{-1}\right)=u\left(s \cdot t^{-1}\right) \\
\mapsto & \widetilde{\mu}\left(\sigma_{2}(\widetilde{\varkappa}(u))\right) \in \mathbb{C}^{G}, \quad \widetilde{\mu}\left(\sigma_{2}(\widetilde{\varkappa}(u))\right)(s)=\sigma_{1}(\widetilde{\varkappa}(u))(s, s)=\widetilde{\varkappa}(u)\left(s, s^{-1}\right)=u\left(s \cdot s^{-1}\right)=u\left(1_{G}\right)
\end{array}
$$

\section{(d) Sweedler's notations and the stereotype approximation property}

A useful instrument for proving results in the theory of Hopf algebra are Sweedler's notations [19. This technique can be applied in the stereotype theory as well, at least in situations, where a given stereotype Hopf algebra $H$, being considered as a stereotype space possesses the stereotype approximation property (see [1]).

The following result explains this:

Theorem 1.8. If $H$ is an injective (respectively, a projective) stereotype coalgebra with the stereotype approximation property, then for any $x \in H$

(i) the comultiplication $\varkappa(x)$ can be approximated in the topology of $H$ by the finite sums of the form

$$
\sum_{i=1}^{n} x_{i}^{\prime} \odot x_{i}^{\prime \prime} \quad\left(\sum_{i=1}^{n} x_{i}^{\prime} \circledast x_{i}^{\prime \prime}\right)
$$

(ii) the identity

$$
\langle\varkappa(x), \alpha \circledast \beta\rangle=0 \quad(\langle\varkappa(x), \alpha \odot \beta\rangle=0), \quad \alpha, \beta \in H^{\star}
$$

is equivalent to the identity

$$
\varkappa(x)=0
$$

Proof. This follows from the definition of the stereotype approximation [1, §9].

If now $H$, say, is an injective stereotype coalgebra with the stereotype approximation property, then for each element $x \in H$ the symbol $\sum_{(x)} x^{\prime} \odot x^{\prime \prime}$ denotes the class of nets of the form $\sum_{i=1}^{n_{\nu}} x_{\nu, i}^{\prime} \odot x_{\nu, i}^{\prime \prime}$ tending to $\varkappa(x)$ :

$$
\varkappa(x) \underset{\infty \leftarrow \nu}{\longleftarrow} \sum_{i=1}^{n_{\nu}} x_{\nu, i}^{\prime} \odot x_{\nu, i}^{\prime \prime}
$$


and the record

$$
\varkappa(x)=\sum_{(x)} x^{\prime} \odot x^{\prime \prime}
$$

should be understood as follows: the right side denotes one of those nets (but without indices), and the arrow is replaced by the equality.

The formulas for comultiplication and antipode, and the others, like

$$
\varkappa(\sigma(x))=\sum_{(x)} \sigma\left(x^{\prime \prime}\right) \odot \sigma\left(x^{\prime}\right)
$$

are interpreted as follows: for every net $\sum_{i=1}^{n_{\nu}} x_{\nu, i}^{\prime} \odot x_{\nu, i}^{\prime \prime}$ the condition (1.32) automatically implies the condition

$$
\varkappa(\sigma(x)) \overleftarrow{\infty \leftarrow \nu} \sum_{i=1}^{n_{\nu}} \sigma\left(x_{\nu, i}^{\prime \prime}\right) \odot \sigma\left(x_{\nu, i}^{\prime}\right)
$$

The proof can also be conducted with the help of the record (1.33):

$$
\begin{array}{r}
\langle\varkappa(\sigma(x)), \alpha \circledast \beta\rangle=\langle\sigma(x), \alpha * \beta\rangle=\left\langle x, \sigma^{\star}(\alpha * \beta)\right\rangle=\left\langle x, \sigma^{\star}(\beta) * \sigma^{\star}(\alpha)\right\rangle=\left\langle\varkappa(x), \sigma^{\star}(\beta) \circledast \sigma^{\star}(\alpha)\right\rangle= \\
=\left\langle\sum_{(x)} x^{\prime} \odot x^{\prime \prime}, \sigma^{\star}(\beta) \circledast \sigma^{\star}(\alpha)\right\rangle=\sum_{(x)}\left\langle x^{\prime} \odot x^{\prime \prime}, \sigma^{\star}(\beta) \circledast \sigma^{\star}(\alpha)\right\rangle=\sum_{(x)}\left\langle x^{\prime}, \sigma^{\star}(\beta)\right\rangle \cdot\left\langle x^{\prime \prime}, \sigma^{\star}(\alpha)\right\rangle= \\
=\sum_{(x)}\left\langle\sigma\left(x^{\prime}\right), \beta\right\rangle \cdot\left\langle\sigma\left(x^{\prime \prime}\right), \alpha\right\rangle=\sum_{(x)}\left\langle\sigma\left(x^{\prime \prime}\right) \odot \sigma\left(x^{\prime}\right), \alpha \circledast \beta\right\rangle=\left\langle\sum_{(x)} \sigma\left(x^{\prime \prime}\right) \odot \sigma\left(x^{\prime}\right), \alpha \circledast \beta\right\rangle
\end{array}
$$

As an example of the application of these "generalized" Sweedler's notations let us consider the following situation. In the theory of quantum groups the verification of the diagram for antipode (1.18), i.e. the identity

$$
\mu((\sigma \otimes 1)(\varkappa(x)))=\varepsilon(x) \cdot 1_{H}=\mu((1 \otimes \sigma)(\varkappa(x))), \quad x \in H
$$

often leads to some bulky computations. In those cases one remark, made by A. Van Daele in [39] is useful. Being applied to stereotype algebras it looks as follows:

Lemma 1.1. Suppose $H$ is a stereotype bialgebra (no matter, projective, or injective) with the stereotype approximation property, $\sigma$ is its (continuous) antihomomorphism and the equalities (1.34) are true for two elements $x \in H$ and $y \in H$. Then they are true for their multiplication $x \cdot y$.

Proof. Both those equalities for $x \cdot y$ are proved by direct computations, for instance, the left one is obtained as follows (here the tensor product $\otimes$ means $\circledast$ or $\odot$ ):

$$
\begin{aligned}
& \mu((\sigma \otimes 1)(\varkappa(x \cdot y)))=\mu((\sigma \otimes 1)(\varkappa(x) \cdot \varkappa(y)))=\mu\left((\sigma \otimes 1)\left(\sum_{(x)} x^{\prime} \otimes x^{\prime \prime} \cdot \sum_{(y)} y^{\prime} \otimes y^{\prime \prime}\right)\right)= \\
& =\sum_{(x),(y)} \mu\left((\sigma \otimes 1)\left(x^{\prime} \cdot y^{\prime} \otimes x^{\prime \prime} \cdot y^{\prime \prime}\right)\right)=\sum_{(x),(y)} \mu\left(\sigma\left(x^{\prime} \cdot y^{\prime}\right) \otimes x^{\prime \prime} \cdot y^{\prime \prime}\right)=\sum_{(x),(y)} \mu\left(\sigma\left(y^{\prime}\right) \cdot \sigma\left(x^{\prime}\right) \otimes x^{\prime \prime} \cdot y^{\prime \prime}\right)= \\
& =\sum_{(x),(y)} \sigma\left(y^{\prime}\right) \cdot \sigma\left(x^{\prime}\right) \cdot x^{\prime \prime} \cdot y^{\prime \prime}=\sum_{(y)} \sigma\left(y^{\prime}\right) \cdot\left(\sum_{(x)} \sigma\left(x^{\prime}\right) \cdot x^{\prime \prime}\right) \cdot y^{\prime \prime}=\sum_{(y)} \sigma\left(y^{\prime}\right) \cdot \varepsilon(x) \cdot 1_{H} \cdot y^{\prime \prime}= \\
& =\varepsilon(x) \cdot 1_{H} \cdot \sum_{(y)} \sigma\left(y^{\prime}\right) \cdot y^{\prime \prime}=\varepsilon(x) \cdot 1_{H} \cdot \varepsilon(y) \cdot 1_{H} \cdot=\varepsilon(x \cdot y) \cdot 1_{H} .
\end{aligned}
$$




\section{(e) Grouplike elements}

Proposition 1.2. For an element $a \in H$ in a stereotype Hopf algebra $H$ with the stereotype approximation property the following conditions are equivalent:

(i) $\varkappa(a)=a \odot a \quad(\varkappa(a)=a \circledast a)$;

(ii) the functional $\langle a, \cdot\rangle: H^{\star} \rightarrow \mathbb{C}$ is multiplicative:

$$
\langle a, \alpha * \beta\rangle=\langle a, \alpha\rangle \cdot\langle a, \beta\rangle
$$

(iii) the operator $\mathrm{M}_{a}^{\star}: H^{\star} \rightarrow H^{\star}$ dual to the operator of multiplication by element a,

$$
\mathrm{M}_{a}(x):=a \cdot x
$$

is a homomorphism of stereotype algebra $H^{\star}$.

Proof. Obviously, (i) and (ii) are equivalent. Let us show that (i) $\Longleftrightarrow$ (iii). If $a$ satisfies (i), then

$$
\begin{array}{r}
\left\langle x, \mathrm{M}_{a}^{\star}(\alpha * \beta)\right\rangle=\left\langle\mathrm{M}_{a}(x), \alpha * \beta\right\rangle=\langle a \cdot x, \alpha * \beta\rangle=\langle\varkappa(a \cdot x), \alpha \circledast \beta\rangle=\langle\varkappa(a) \cdot \varkappa(x), \alpha \circledast \beta\rangle= \\
=\left\langle a \odot a \cdot \sum_{(x)} x^{\prime} \odot x^{\prime \prime}, \alpha \circledast \beta\right\rangle=\left\langle\sum_{(x)}\left(a \cdot x^{\prime}\right) \odot\left(a \cdot x^{\prime \prime}\right), \alpha \circledast \beta\right\rangle=\sum_{(x)}\left\langle a \cdot x^{\prime}, \alpha\right\rangle \cdot\left\langle a \cdot x^{\prime \prime}, \beta\right\rangle= \\
=\sum_{(x)}\left\langle x^{\prime}, \mathrm{M}_{a}^{\star}(\alpha)\right\rangle \cdot\left\langle x^{\prime \prime}, \mathrm{M}_{a}^{\star}(\beta)\right\rangle=\left\langle\sum_{(x)} x^{\prime} \odot x^{\prime \prime}, \mathrm{M}_{a}^{\star}(\alpha) \circledast \mathrm{M}_{a}^{\star}(\beta)\right\rangle=\left\langle\varkappa(x), \mathrm{M}_{a}^{\star}(\alpha) \circledast \mathrm{M}_{a}^{\star}(\beta)\right\rangle= \\
=\left\langle x, \mathrm{M}_{a}^{\star}(\alpha) * \mathrm{M}_{a}^{\star}(\beta)\right\rangle
\end{array}
$$

This is true for every $x \in H$, therefore

$$
\mathrm{M}_{a}^{\star}(\alpha * \beta)=\mathrm{M}_{a}^{\star}(\alpha) * \mathrm{M}_{a}^{\star}(\beta)
$$

On the contrary, if (1.36) is true, then

$$
\begin{aligned}
\langle\varkappa(a), \alpha \circledast \beta\rangle= & \langle a, \alpha * \beta\rangle=\left\langle 1, \mathrm{M}_{a}^{\star}(\alpha * \beta)\right\rangle=\left\langle 1, \mathrm{M}_{a}^{\star}(\alpha) * \mathrm{M}_{a}^{\star}(\beta)\right\rangle=\left\langle\varkappa(1), \mathrm{M}_{a}^{\star}(\alpha) \circledast \mathrm{M}_{a}^{\star}(\beta)\right\rangle= \\
& =\left\langle 1 \odot 1, \mathrm{M}_{a}^{\star}(\alpha) \circledast \mathrm{M}_{a}^{\star}(\beta)\right\rangle=\left\langle 1, \mathrm{M}_{a}^{\star}(\alpha)\right\rangle \cdot\left\langle 1, \mathrm{M}_{a}^{\star}(\beta)\right\rangle=\langle a, \alpha\rangle \cdot\langle a, \beta\rangle=\langle a \odot a, \alpha \circledast \beta\rangle
\end{aligned}
$$

and since this is true for each $\alpha$ and $\beta$, we obtain (i).

An element $a$ of an injective (resp., projective) stereotype Hopf algebra $H$ is called grouplike element, if $a \neq 0$ and $a$ satisfies the conditions (i)-(ii)-(iii) of Proposition 1.2 The set of grouplike elements in $H$ is denoted by $\mathrm{G}(H)$. As in the pure algebraic situation (see [37]), $\mathrm{G}(H)$ is a group with respect to the multiplication in $H$, since it possesses the following properties:

$$
\begin{aligned}
& 1^{\circ} . \forall a \in \mathrm{G}(H) \quad \varepsilon(a)=1 . \\
& 2^{\circ} . \forall a \in \mathrm{G}(H) \quad a^{-1}=\sigma(a) \in \mathrm{G}(H) . \\
& 3^{\circ} . \forall a, b \in \mathrm{G}(H) \quad a \cdot b \in \mathrm{G}(H) .
\end{aligned}
$$

Proof. $1^{\circ}$ is proved by applying the comultiplication axiom:

$$
1 \odot a=\mathrm{I}_{H}^{-1}(a)=(\underline{1.14})=\left(\varepsilon \odot \mathrm{id}_{H}\right)(\varkappa(a))=\left(\varepsilon \odot \mathrm{id}_{H}\right)(a \odot a)=\varepsilon(a) \odot a \quad \Longrightarrow \quad \varepsilon(a)=1
$$

In $2^{\circ}$ we need to apply the fact that $\sigma^{\star}$ is an antihomomorphism: on the one hand, $\varkappa(\sigma(a))=\sigma(a) \odot \sigma(a)$, since 


$$
\begin{aligned}
& \langle\varkappa(\sigma(a)), \alpha \circledast \beta\rangle=\langle\sigma(a), \alpha * \beta\rangle=\left\langle a, \sigma^{\star}(\alpha * \beta)\right\rangle=\left\langle a, \sigma^{\star}(\beta) * \sigma^{\star}(\alpha)\right\rangle=\left\langle\varkappa(a), \sigma^{\star}(\beta) \circledast \sigma^{\star}(\alpha)\right\rangle= \\
& \quad=\left\langle a \odot a, \sigma^{\star}(\beta) \circledast \sigma^{\star}(\alpha)\right\rangle=\left\langle a, \sigma^{\star}(\beta)\right\rangle \cdot\left\langle a, \sigma^{\star}(\alpha)\right\rangle=\langle\sigma(a), \beta\rangle \cdot\langle\sigma(a), \alpha\rangle=\langle\sigma(a) \odot \sigma(a), \alpha \circledast \beta\rangle
\end{aligned}
$$

And, on the other hand, $\varepsilon(\sigma(a))=\left\langle\sigma(a), 1_{H}\right\rangle=\left\langle a, \sigma^{\star}\left(1_{H}\right)\right\rangle=\left\langle a, 1_{H}\right\rangle=\varepsilon(a)=1$. Together these conditions mean that $\sigma(a) \in \mathrm{G}(H)$. Apart from that,

$\sigma(a) \cdot a=\mu(\sigma(a) \odot a)=\mu\left(\left(\sigma \odot \operatorname{id}_{H}\right)(a \odot a)\right)=\mu\left(\left(\sigma \odot \operatorname{id}_{H}\right)(\varkappa(a))\right)=(1.18)=\varepsilon(a) \cdot 1_{H}=\left(\right.$ property $\left.^{\circ}\right)=1_{H}$ and, similarly, $a \cdot \sigma(a)=1_{H}$. Hence, $\sigma(a)=a^{-1}$.

Finally, $3^{\circ}:$ if $a, b \in \mathrm{G}(H)$, then, first, $\varkappa(a \cdot b)=\varkappa(a) \cdot \varkappa(b)=a \odot a \cdot b \odot b=(a \cdot b) \odot(a \cdot b)$, and, second, $\varepsilon(a \cdot b)=\varepsilon(a) \cdot \varepsilon(b)=1$. Therefore, $a \cdot b \in \mathrm{G}(H)$.

Recall that an element $a$ of an algebra $A$ is called central, if it commutes with all other elements of $A$ :

$$
\forall x \in A \quad a \cdot x=x \cdot a
$$

Proposition 1.3. If $a$ is a grouplike and in addition a central element in a Hopf algebra $H$, then

1) the following identities hold:

$$
\begin{array}{ll}
\mathrm{M}_{a} \circ \sigma \circ \mathrm{M}_{a}=\sigma, & \sigma \circ \mathrm{M}_{a}=\mathrm{M}_{a^{-1}} \circ \sigma \\
\mathrm{M}_{a}^{\star} \circ \sigma^{\star} \circ \mathrm{M}_{a}^{\star}=\sigma^{\star}, & \sigma^{\star} \circ \mathrm{M}_{a}^{\star}=\mathrm{M}_{a^{-1}}^{\star} \circ \sigma^{\star}
\end{array}
$$

2) if $H$ has the stereotype approximation property, then the following identities hold:

$$
\begin{gathered}
\varkappa\left(\mathrm{M}_{a}^{\star}(\alpha)\right)=\sum_{(\alpha)} \mathrm{M}_{a}^{\star}\left(\alpha^{\prime}\right) \circledast \alpha^{\prime \prime}=\sum_{(\alpha)} \alpha^{\prime} \circledast \mathrm{M}_{a}^{\star}\left(\alpha^{\prime \prime}\right), \quad \alpha \in H^{\star} \\
\varkappa\left(\left(\mathrm{M}_{a}^{\star}\right)^{i+j}(\alpha)\right)=\sum_{(\alpha)}\left(\mathrm{M}_{a}^{\star}\right)^{i}\left(\alpha^{\prime}\right) \circledast\left(\mathrm{M}_{a}^{\star}\right)^{j}\left(\alpha^{\prime \prime}\right), \quad \alpha \in H^{\star}, \quad i, j \in \mathbb{N}
\end{gathered}
$$

Proof. 1. For each $x \in H$ we have

$$
\left(\mathrm{M}_{a} \circ \sigma \circ \mathrm{M}_{a}\right)(x)=a \cdot \sigma(a \cdot x)=a \cdot \sigma(x) \cdot \sigma(a)=a \cdot \sigma(x) \cdot a^{-1}=\sigma(x) \cdot a \cdot a^{-1}=\sigma(x)
$$

2. For any $u, v \in H, \alpha \in H^{\star}$

$$
\begin{aligned}
\left\langle u \odot v, \varkappa\left(\mathrm{M}_{a}^{\star}(\alpha)\right)\right\rangle=\left\langle u \cdot v, \mathrm{M}_{a}^{\star}(\alpha)\right\rangle=\langle a \cdot u \cdot v, \alpha\rangle=\langle(a \cdot u) \odot v, \varkappa(\alpha)\rangle & =\left\langle(a \cdot u) \odot v, \sum_{(\alpha)} \alpha^{\prime} \circledast \alpha^{\prime \prime}\right\rangle= \\
=\sum_{(\alpha)}\left\langle(a \cdot u) \odot v, \alpha^{\prime} \circledast \alpha^{\prime \prime}\right\rangle=\sum_{(\alpha)}\left\langle(a \cdot u), \alpha^{\prime}\right\rangle \cdot\left\langle v, \alpha^{\prime \prime}\right\rangle=\sum_{(\alpha)}\left\langle u, \mathrm{M}_{a}^{\star}\left(\alpha^{\prime}\right)\right\rangle \cdot\left\langle v, \alpha^{\prime \prime}\right\rangle & =\sum_{(\alpha)}\left\langle u \odot v, \mathrm{M}_{a}^{\star}\left(\alpha^{\prime}\right) \circledast \alpha^{\prime \prime}\right\rangle= \\
& =\left\langle u \odot v, \sum_{(\alpha)} \mathrm{M}_{a}^{\star}\left(\alpha^{\prime}\right) \circledast \alpha^{\prime \prime}\right\rangle
\end{aligned}
$$

By Theorem 1.8 this means that the first equality in (1.39) holds. The rest equalities are proved by analogy.

\section{$\S 2$ Stein manifolds: rectangles in $\mathcal{O}(M)$ and rhombuses in $\mathcal{O}^{\star}(M)$}

Here we discuss some special properties of the space of holomorphic functions on a complex manifold. For illustration purposes it is convenient for us to use the condition of holomorphic separability, so we formulate our results only for Stein manifolds. We use terminology from [34, 38, 14. 


\section{(a) Stein manifolds}

Let $M$ be a complex manifold. Symbol $\mathcal{O}(M)$ denotes the algebra of all holomorphic functions on $M$ (with the usual pointwise algebraic operations and topology of uniform convergence on compact sets in $M)$. It is well-known ( see [38), that as a topological vector space, $\mathcal{O}(M)$ is a Montel space.

A manifold $M$ is called a Stein manifold [34, if the following three conditions are fulfilled:

1) holomorphic separability: for any two points $x, y \in M, x \neq y$, there exists a function $u \in \mathcal{O}(M)$ such that

$$
u(x) \neq u(y)
$$

2) holomorphic uniformization: for any point $x \in M$ there exist functions $u_{1}, \ldots, u_{n} \in \mathcal{O}(M)$, forming local coordinates of the manifold $M$ in a neighborhood of $x$;

3) holomorphic convexity: for any compact set $K \subseteq M$ its holomorphically convex hull, i.e. a the set

$$
\hat{K}=\left\{x \in M: \forall u \in \mathcal{O}(M)|u(x)| \leqslant \max _{y \in K}|u(y)|\right\}
$$

is a compact set in $M$.

The complex space $\mathbb{C}^{n}$ and various domains of holomorphy in $\mathbb{C}^{n}$ are examples of Stein manifolds. A simplest example of a complex manifold which is not a Stein manifold is complex torus, i.e. the quotient group of the complex plane $\mathbb{C}$ over the lattice $\mathbb{Z}+i \mathbb{Z}$.

\section{(b) Outer envelopes on $M$ and rectangles in $\mathcal{O}(M)$}

Operations $\square$ and $\square$. Here we shall spend some time on studying real functions $f$ on a manifold $M$, bounded by 1 from below,

$$
f \geqslant 1
$$

i.e. $f$ having range in the interval $[1,+\infty)$. Certainly, we shall use record $f: M \rightarrow[1 ;+\infty)$ for such functions. As usual, we call a function $f: M \rightarrow[1 ;+\infty)$ locally bounded, if for each point $x \in M$ one can find a neighborhood $U \ni x$ such that

$$
\sup _{y \in U}|f(y)|<\infty
$$

Since $f$ is bounded by 1 from below, this condition is equivalent to the condition

$$
\sup _{y \in U} f(y)<\infty
$$

Proposition 2.1. For each locally bounded function $f: M \rightarrow[1 ;+\infty)$ the formula

$$
f \mathbf{\amalg}:=\{u \in \mathcal{O}(M): \quad \forall x \in M \quad|u(x)| \leqslant f(x)\}
$$

defines an absolutely convex set of functions $f \subseteq \mathcal{O}(M)$, containing the identity function:

$$
1 \in f^{\square} \text {. }
$$

Proof. The set $f \mathbf{\square}$ is compact since it is closed and bounded in the Montel space $\mathcal{O}(M)$.

Proposition 2.2. For any bounded set of functions $D \subseteq \mathcal{O}(M)$ containing the identity function,

$$
1 \in D
$$

the formula

$$
D^{\square}(x):=\sup _{u \in D}|u(x)|, \quad x \in M
$$

defines a continuous real function $D^{\square}: M \rightarrow \mathbb{R}$ bounded by 1 from below:

$$
D^{\square} \geqslant 1 \text {. }
$$


Proof. Note from the very beginning that $D$ can be considered as compact. Consider for this its closure $\bar{D}$. Since $D$ is bounded in a Montel space $\mathcal{O}(M), \bar{D}$ is compact in $\mathcal{O}(M)$. The mapping $u \mapsto \delta^{x}(u)=u(x)$ is continuous, so the image of the closure $\delta^{x}(\bar{D})$ is contained in the closure of image $\overline{\delta^{x}(D)}$

$$
\delta^{x}(\bar{D}) \subseteq \overline{\delta^{x}(D)},
$$

As a corollary, we obtain the following chain of inequalities:

$$
\sup _{\lambda \in \delta^{x}(D)}|\lambda| \leqslant \sup _{\lambda \in \delta^{x}(\bar{D})}|\lambda| \leqslant \sup _{\lambda \in \overline{\delta^{x}(D)}}|\lambda|=\sup _{\lambda \in \delta^{x}(D)}|\lambda|
$$

Therefore,

$$
\sup _{\lambda \in \delta^{x}(\bar{D})}|\lambda|=\sup _{\lambda \in \delta^{x}(D)}|\lambda|
$$

and the functions $\bar{D}$ and $D^{\square}$ coincide:

$$
\bar{D} \square(x)=\sup _{u \in \bar{D}}\left|\delta^{x}(u)\right|=\sup _{\lambda \in \delta^{x}(\bar{D})}|\lambda|=\sup _{\lambda \in \delta^{x}(D)}|\lambda|=\sup _{u \in D}\left|\delta^{x}(u)\right|=D^{\square}(x)
$$

Thus it is sufficient to consider the case when $D$ is compact. Let us take an arbitrary compact set $K \subseteq M$ and consider the space $C(K)$ of continuous functions on $K$ (with the usual topology of uniform convergence on $K)$. The restriction mapping $\left.u \in \mathcal{O}(M) \mapsto u\right|_{K} \in C(K)$ is continuous from $\mathcal{O}(M)$ into $C(K)$, so the image $\left.D\right|_{K}$ of a compact $D$ in $\mathcal{O}(M)$ must be compact in $C(K)$. Hence, by the Arcela theorem $\left.D\right|_{K}$, is pointwise bounded and equicontinuous on $K$. Therefore the function

$$
D^{\square}(x):=\sup _{u \in D}|u(x)|, \quad x \in K
$$

is continuous on $K$. This is true for every compact set $K$ in $M$, so we obtain that $D^{\square}$ is continuous on $M$.

\section{Properties of operations \ and $\square$ :}

$$
\begin{aligned}
& f \leqslant g \quad \Longrightarrow \quad f^{\mathbf{\bullet}} \subseteq g^{\mathbf{\downarrow}}, \\
& D \subseteq E \quad \Longrightarrow \quad D^{\square} \leqslant E^{\square} \\
& \left(f^{\square}\right)^{\square} \leqslant f, \\
& D \subseteq\left(D^{\square}\right)
\end{aligned}
$$

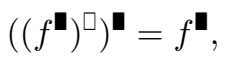

$$
\begin{aligned}
& \left(\left(D^{\square}\right)^{\square}\right)^{\square}=D^{\square}
\end{aligned}
$$

Proof. Properties (2.3) and (2.4) are evident, and (2.5) follows from them:

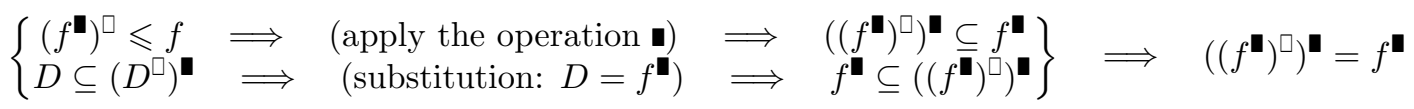

$$
\begin{aligned}
& \left\{\begin{array}{ccccc}
D \subseteq\left(D^{\square}\right) & \Longrightarrow & \text { (apply the operation } \square) & \Longrightarrow & D^{\square} \leqslant\left(\left(D^{\square}\right)^{\square}\right)^{\square} \\
(f)^{\square} \leqslant f & \Longrightarrow & \text { (substitution: } \left.f=D^{\square}\right) & \Longrightarrow & \left(\left(D^{\square}\right)^{\square} \leqslant D^{\square}\right.
\end{array}\right\} \quad \Longrightarrow \quad\left(\left(D^{\square}\right)^{\square}\right)^{\square}=D^{\square}
\end{aligned}
$$


Outer envelopes on $M$. Let us introduce the following notations:

$$
f^{\mathbf{\square}}:=\left(f^{\mathbf{\square}}\right)^{\square} \quad D^{\square \mathbf{\square}}:=\left(D^{\square}\right)^{\mathbf{\square}}
$$

Then (2.3), (2.4), and (2.5) imply

$$
\begin{aligned}
& f^{\square} \leqslant f, \\
& D \subseteq D^{\square \square} \\
& f \leqslant g \quad \Longrightarrow \quad f^{\square} \leqslant g^{\square}, \\
& D \subseteq E \quad \Longrightarrow \quad D^{\square \mathbf{I}} \subseteq E^{\square \mathbf{\square}} \\
& \left(f^{\square}\right)^{\square}=f^{\square} \\
& \left(D^{\square \mathbf{\square}}\right)^{\square \mathbf{\square}}=D^{\square \mathbf{\square}}
\end{aligned}
$$

Let us call a locally bounded function $g: M \rightarrow[1,+\infty)$,

- an outer envelope for a bounded set $D \subseteq \mathcal{O}(M), 1 \in D$, if

$$
g=D^{\square}
$$

- an outer envelope for a locally bounded function $f: M \rightarrow[1 ;+\infty)$, if

$$
g=f^{\mathbf{\square}}
$$

- an outer envelope on $M$, if it satisfies the following equivalent conditions:

(i) $g$ is an outer envelope for some bounded set $D \subseteq \mathcal{O}(M), 1 \in D$,

$$
g=D^{\square}
$$

(ii) $g$ is an outer envelope for some locally bounded function $f: M \rightarrow[1 ;+\infty)$,

$$
g=f^{\square}
$$

(iii) $g$ is an outer envelope for some for itself:

$$
g^{\square}=g
$$

Proof. The equivalence of conditions (i), (ii), (iii) requires some comments.

$(i) \Longrightarrow(i i)$. If $g$ is an outer envelope for some bounded set $D$, i.e. $g=D^{\square}$, then $g=D^{\square}=(2.5)=$ $\left(\left(D^{\square}\right)^{\square}=\left(D^{\square}\right)^{\square}\right.$, i.e. $g$ is an outer envelope for the function $f=D^{\square}$.

$($ ii $) \Longrightarrow($ iii $)$. If $g$ is an outer envelope for some function $f$, i.e. $g=f^{\mathbf{\square}}$, then $g^{\square}=\left(f^{\square}\right)^{\square}=(2.9)=$ $f \square=g$, i.e. $g$ is an outer envelope for itself.

$($ iii $) \Longrightarrow($ i $)$. If $g$ is an outer envelope for itself, i.e. $g=g^{\square}=\left(g^{\square}\right)^{\square}$, then we put $D=g^{\mathbf{\square}}$, and after that $g$ becomes an outer envelope for $D: g=D^{\square}$.

\section{Properties of outer envelopes:}

(i) Every outer envelope $g$ on $M$ is a continuous (positive) function on $M$.

(ii) For any locally bounded function $f: M \rightarrow[1 ;+\infty)$ its outer envelope $f^{\square}$ is the greatest outer envelope on $M$, majorized by $f$ :

(a) $f \square$ is an outer envelope on $M$, majorized by $f$ :

$$
f^{\square} \leqslant f
$$

(b) if $g$ is another outer envelope on $M$, majorized by $f$,

$$
g \leqslant f
$$

then $g$ is majorized by $f^{\square}$ :

$$
g \leqslant f^{\square}
$$


Rectangles in $\mathcal{O}(M)$. We call a set $E \subseteq \mathcal{O}(M), 1 \in E$,

- a rectangle, generated by a locally bounded function $f: M \rightarrow[1 ;+\infty)$, if

$$
E=f
$$

- a rectangle, generated by a bounded set $D \subseteq \mathcal{O}(M), 1 \in D$, if

$$
E=D^{\square \mathbf{U}}
$$

- a rectangle in $\mathcal{O}(M)$, if the following equivalent conditions hold:

(i) $E$ is a rectangle, generated by some locally bounded function $f: M \rightarrow[1 ;+\infty)$

$$
E=f
$$

(ii) $E$ is a rectangle, generated by some bounded set $D \subseteq \mathcal{O}(M), 1 \in D$,

$$
E=D^{\square \mathbf{r}}
$$

(iii) $E$ is a rectangle, generated by itself:

$$
E=E^{\square}
$$

Proof. The equivalence of conditions (i), (ii), (iii) is proved in the same way as in the case of outer envelopes.

\section{Properties of rectangles:}

(i) Every rectangle $\mathrm{E}$ in $\mathcal{O}(M)$ is an absolutely convex compact set in $\mathcal{O}(M)$.

(ii) For any bounded set $D \subseteq \mathcal{O}(M)$ the rectangle $D^{\square}$ is the smallest rectangle in $\mathcal{O}(M)$, containing $D$ :

(a) $D^{\square}$ is a rectangle in $\mathcal{O}(M)$, containing $D$ :

$$
D \subseteq D^{\square \mathbf{\square}}
$$

(b) if $E$ is another rectangle in $\mathcal{O}(M)$, containing $D$,

$$
D \subseteq E,
$$

then $E$ contains $D^{\square \cdot}$ :

$$
D^{\square \mathbf{\square}} \subseteq E
$$

(iii) The rectangles in $\mathcal{O}(M)$ form a fundamental system of compact sets in $\mathcal{O}(M)$ : every compact set $D$ in $\mathcal{O}(M)$ is contained in some rectangle.

Theorem 2.1. The formulas

$$
D=f^{\llbracket}, \quad f=D^{\square}
$$

establish a bijection between outer envelopes $f$ on $M$ and rectangles $D$ in $\mathcal{O}(M)$.

Proof. By definition of outer envelopes and rectangles, the operations $f \mapsto f \boldsymbol{\Downarrow}^{\text {and }} D \mapsto D^{\square}$ turn outer envelopes into rectangles and rectangles into outer envelopes. Moreover, these operations are mutually inverse on those two classes - if $f$ is an outer envelope, then $f \square=f$, i.e. the composition of the operations - and $\square$ gives back to the initial function:

$$
f \mapsto f^{\mathbf{\bullet}} \mapsto f^{\mathbf{\square}}=f
$$

Just like this the composition of the operations $\square$ and returns to the set $D$, if initially it was chosen as a rectangle:

$$
D \mapsto D^{\square} \mapsto D^{\square \mathbf{r}}=D
$$




\section{(c) Lemma on polars}

Recall that polar of a set $A$ in a locally convex space $X$ is the set $A^{\circ}$ of linear continuous functionals $f: X \rightarrow \mathbb{C}$, bounded by 1 on $A$ :

$$
A^{\circ}=\left\{f \in X^{\star}: \quad \sup _{x \in A}|f(x)| \leqslant 1\right\}
$$

If $X$ is a stereotype space and $A$ a subset in the dual space $X^{\star}$, then because of the equality $\left(X^{\star}\right)^{\star}=X$ it is convenient to consider the polar $A^{\circ} \subseteq\left(X^{\star}\right)^{\star}$ as a subset in $X$. We denote this set by ${ }^{\circ} A$ (and again call it polar of $A$ ):

$$
{ }^{\circ} A=\left\{x \in X: \quad \sup _{f \in A}|f(x)| \leqslant 1\right\}
$$

An important observation for us is that if $A \subseteq X^{\star}$, and we take its polar ${ }^{\circ} A$, and after that "the polar of the polar" $\left({ }^{\circ} A\right)^{\circ}$ - this set is called bipolar of $A$ - then it turns out that $\left({ }^{\circ} A\right)^{\circ}$ is exactly the closed absolutely convex hull of the set $A$ in $X^{\star}$ (i.e. the closure of the set of linear combinations of the form $\sum_{i=1}^{n} \lambda_{i} \cdot a_{i}$, where $\left.a_{i} \in A, \sum_{i=1}^{n}\left|\lambda_{i}\right| \leqslant 1\right)$ :

$$
\left({ }^{\circ} A\right)^{\circ}=\overline{\operatorname{absconv}} A
$$

That is the essence the classical theorem on bipolar as applied to stereotype spaces.

In the special case when $A=D$ is a set in $\mathcal{O}(M)$, its polar $D^{\circ}$ is the set of analytical functionals $\alpha \in \mathcal{O}^{\star}(M)$, bounded by 1 on $D$ :

$$
D^{\circ}=\left\{\alpha \in \mathcal{O}^{\star}(M): \quad \sup _{u \in D}|\alpha(u)| \leqslant 1\right\}
$$

On the contrary, if $A$ is a set of analytical functionals, $A \subseteq \mathcal{O}^{\star}(M)$, then its polar ${ }^{\circ} A$ in $\mathcal{O}(M)$ is the set of functions $u \in \mathcal{O}(M)$ on which all the functionals $\alpha \in A$ are bounded by 1 :

$$
{ }^{\circ} A=\left\{u \in \mathcal{O}(M): \quad \sup _{\alpha \in A}|\alpha(u)| \leqslant 1\right\}
$$

Lemma 2.1 (on polars). The passage to polars possesses the following properties.

(a) For every bounded set $D$ in $\mathcal{O}(M)$ containing the unit, its outer envelope $D^{\square}$ is connected with its polar $D^{\circ}$ through the identity

$$
\frac{1}{D^{\square}(x)}=\max \left\{\lambda>0: \quad \lambda \cdot \delta^{x} \in D^{\circ}\right\}
$$

(b) For any locally bounded function $f: M \rightarrow[1 ;+\infty)$ the rectangle $f$ is the polar of functionals $\frac{1}{f(x)} \cdot \delta^{x}:$

$$
f \boldsymbol{\square}={ }^{\circ}\left\{\frac{1}{f(x)} \cdot \delta^{x} ; \quad x \in M\right\}
$$

(c) The polar of the rectangle $f$ is an absolutely convex hull of functionals $\frac{1}{f(x)} \cdot \delta^{x}$ :

$$
\left(f^{\mathbf{\varpi}}\right)^{\circ}=\overline{\operatorname{absconv}}\left\{\frac{1}{f(x)} \cdot \delta^{x} ; \quad x \in M\right\}
$$

Proof. (a) For $\lambda>0$ we have:

$$
\lambda \cdot \delta^{x} \in D^{\circ} \quad \Longleftrightarrow \sup _{u \in D}\left|\lambda \cdot \delta^{x}(u)\right| \leqslant 1 \quad \Longleftrightarrow \quad D^{\square}(x)=\sup _{u \in D}\left|\delta^{x}(u)\right| \leqslant \frac{1}{\lambda} \quad \Longleftrightarrow \quad \lambda \leqslant \frac{1}{D^{\square}(x)}
$$


(b) is a reformulation of the definition of $f \mathbf{~}$

$$
\begin{aligned}
u \in f \stackrel{(2.1)}{\Longleftrightarrow} \forall x \in M \quad|u(x)| & =\left|\delta^{x}(u)\right| \leqslant f(x) \quad \Longleftrightarrow \\
& \Longleftrightarrow \sup _{x \in M}\left|\frac{1}{f(x)} \cdot \delta^{x}(u)\right| \leqslant 1 \quad \Longleftrightarrow u \in \in^{\circ}\left\{\frac{1}{f(x)} \cdot \delta^{x} ; \quad x \in M\right\}
\end{aligned}
$$

(c) follows from (b) and from the theorem on bipolar:

$$
(f)^{\circ}=\left(\circ\left\{\frac{1}{f(x)} \cdot \delta^{x} ; \quad x \in M\right\}\right)^{\circ}=\left(\underline{2.11)}=\overline{\operatorname{absconv}}\left\{\frac{1}{f(x)} \cdot \delta^{x} ; \quad x \in M\right\}\right.
$$

\section{(d) Inner envelopes on $M$ and rhombuses in $\mathcal{O}^{\star}(M)$}

In this subsection we shall study closed absolutely convex neighborhoods of zero $\Delta$ in $\mathcal{O}^{\star}(M)$, satisfying the following two equivalent conditions:

(A) the polar ${ }^{\circ} \Delta$ of $\Delta$ contains the unit $1 \in \mathcal{O}(M)$ :

$$
1 \in{ }^{\circ} \Delta
$$

(B) the value of all functionals $\alpha \in \Delta$ on the unit $1 \in \mathcal{O}(M)$ does not exceed 1:

$$
\forall \alpha \in \Delta \quad|\alpha(1)| \leqslant 1
$$

These conditions imply another one (which is not, however, equivalent to (A) and (B)):

(C) a functional of the form $\lambda \cdot \delta^{x}$, where $\lambda>0$, can belong to $\Delta$ only if $\lambda \leqslant 1$ :

$$
\forall x \in M \quad \forall \lambda>0 \quad\left(\lambda \cdot \delta^{x} \in \Delta \Longrightarrow \lambda \leqslant 1\right) .
$$

Proof. If $\lambda \cdot \delta^{x} \in \Delta$, then $\forall u \in{ }^{\circ} \Delta\left|\lambda \cdot \delta^{x}(u)\right| \leqslant 1$. In particular, for $u=1$ we have $\left|\lambda \cdot \delta^{x}(1)\right|=\lambda \cdot 1 \leqslant 1$, i.e. $\lambda \leqslant 1$.

We say that a function $\varphi: M \rightarrow(0,+\infty)$ is locally separated from zero, if for each point $x \in M$ there exists a neighborhood $U \ni x$ such that

$$
\inf _{y \in U} \varphi(y)>0
$$

\section{Operations $\diamond$ and $\diamond$.}

Proposition 2.3. If a function $\varphi: M \rightarrow(0,1]$ is locally separated from zero, then the formula

$$
\varphi^{\downarrow}:=\overline{\operatorname{absconv}}\left\{\varphi(x) \cdot \delta^{x} ; x \in M\right\}
$$

defines an absolutely convex neighborhood of zero in the space of analytical functionals $\mathcal{O}^{\star}(M)$, satisfying (2.15) - (2.17)

Proof. The function $f(x)=\frac{1}{\varphi(x)}$ is locally bounded and has values in $[1 ;+\infty)$. Hence, by Lemma 2.1,

$$
(f)^{\circ}=(\underline{2.14})=\overline{\operatorname{absconv}}\left\{\frac{1}{f(x)} \cdot \delta^{x} ; \quad x \in M\right\}=\overline{\operatorname{absconv}}\left\{\varphi(x) \cdot \delta^{x} ; \quad x \in M\right\}
$$

This set is a closed absolutely convex neighborhood of zero in $\mathcal{O}^{\star}(M)$, since it is the polar of the com-

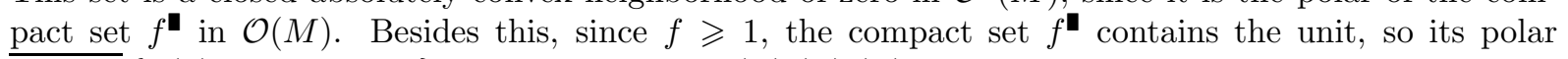
$\overline{\operatorname{absconv}}\left\{\varphi(x) \cdot \delta^{x} ; \quad x \in M\right\}$ satisfies conditions (A),(B),(C) on page 43 
Proposition 2.4. For any closed absolutely convex neighborhood of zero $\Delta$ in the space of analytical functionals $\mathcal{O}^{\star}(M)$, satisfying (2.15) -(2.16) the formula

$$
\Delta^{\diamond}(x):=\sup \left\{\lambda>0: \lambda \cdot \delta^{x} \in \Delta\right\}
$$

defines a continuous and locally separated from zero function $\Delta^{\diamond}: M \rightarrow(0 ; 1]$.

Proof. Since $\Delta$ is a neighborhood of zero in $\mathcal{O}^{\star}(M)$, its polar $D={ }^{\circ} \Delta$ is a compact in $\mathcal{O}^{\star}(M)$, and from (2.15) we have $1 \in D$. By Proposition 2.2, the outer envelope $D^{\square}$ of this compact set is continuous and locally bounded. Hence, the function

$$
\Delta^{\diamond}(x):=\sup \left\{\lambda>0: \lambda \cdot \delta^{x} \in \Delta=D^{\circ}\right\}=(\underline{2.12})=\frac{1}{D^{\square}(x)}
$$

is continuous and locally separated from zero.

The following properties are proved similarly with (2.3), (2.4) and (2.5).

\section{Properties of the operations $\bullet$ and $\diamond:$}

$$
\begin{aligned}
& \varphi \leqslant \psi \quad \Longrightarrow \quad \varphi^{\diamond} \subseteq \psi^{\diamond}, \quad \Delta \subseteq \Gamma \quad \Longrightarrow \quad \Delta^{\diamond} \leqslant \Gamma^{\diamond} \\
& \varphi \leqslant\left(\varphi^{\diamond}\right)^{\diamond}, \quad\left(\Delta^{\diamond}\right)^{\diamond} \subseteq \Delta \\
& \left(\left(\varphi^{\diamond}\right)^{\diamond}\right)^{\diamond}=\varphi^{\diamond}, \quad\left(\left(\Delta^{\diamond}\right)^{\diamond}\right)^{\diamond}=\Delta^{\diamond}
\end{aligned}
$$

Inner envelopes on $M$. Let us use the following supplementary notations:

$$
\varphi^{\diamond}:=\left(\varphi^{\diamond}\right)^{\diamond} \quad \Delta^{\diamond}:=\left(\Delta^{\diamond}\right)^{\diamond}
$$

Formulas (2.3), (2.4), (2.5) imply

$$
\begin{aligned}
& \varphi \leqslant \psi \quad \Longrightarrow \quad \varphi^{\diamond} \leqslant \psi \diamond, \quad \Delta \subseteq \Gamma \quad \Longrightarrow \quad \Delta^{\diamond} \subseteq \Gamma^{\diamond} \\
& \varphi \leqslant \varphi \diamond, \quad \Delta^{\diamond} \subseteq \Delta \\
& \left(\varphi^{\diamond}\right)^{\diamond}=\varphi^{\diamond} \quad\left(\Delta^{\diamond}\right)^{\diamond}=\Delta^{\diamond}
\end{aligned}
$$

Let us call a locally separated from zero function $\psi: M \rightarrow(0 ; 1]$

- an inner envelope for an absolutely convex neighborhood of zero $\Delta$ in $\mathcal{O}^{\star}(M), 1 \in{ }^{\circ} \Delta$, if

$$
\psi=\Delta^{\diamond}
$$

— an inner envelope for a locally separated from zero function $\varphi: M \rightarrow(0 ; 1]$, if

$$
\psi=\varphi \diamond
$$

- an inner envelope on $M$, if it satisfies the following equivalent conditions:

(i) $\psi$ is an inner envelope for some absolutely convex neighborhood of zero $\Delta$ in $\mathcal{O}^{\star}(M), 1 \in{ }^{\circ} \Delta$,

$$
\psi=\Delta^{\diamond}
$$


(ii) $\psi$ is an inner envelope for some locally separated from zero function $\varphi: M \rightarrow(0 ; 1]$.

$$
\psi=\varphi
$$

(iii) $\psi$ is an inner envelope for itself:

$$
\psi \diamond \vee \psi
$$

The equivalence of the conditions (i), (ii), (iii) is proved in the same way as for outer envelopes.

\section{Properties of the inner envelopes:}

(i) Every inner envelope $\psi$ on $M$ is a continuous function on $M$.

(ii) For any locally separated from zero function $\varphi: M \rightarrow(0 ; 1]$ its inner envelope $\varphi$ is the least inner envelope on $M$, majorizing $\varphi$ :

(a) $\varphi$ is an inner envelope on $M$, majorizing $\varphi$ :

$$
\varphi \leqslant \varphi
$$

(b) if $\psi$ is another inner envelope on $M$, majorizing $\varphi$,

$$
\varphi \leqslant \psi
$$

then $\psi$ majorizes $\varphi^{\diamond}$ :

$$
\varphi \leqslant \psi
$$

Rhombuses in $\mathcal{O}^{\star}(M) \quad$ We call a set $\Gamma \subseteq \mathcal{O}^{\star}(M)$,

- a rhombus, generated by a locally separated from zero function $\varphi: M \rightarrow(0 ; 1]$, if

$$
\Gamma=\varphi
$$

- a rhombus, generated by an absolutely convex neighborhood of zero $\Delta \subseteq \mathcal{O}^{\star}(M), 1 \in{ }^{\circ} \Delta$, if

$$
\Gamma=\Delta^{\diamond}
$$

— a rhombus in $\mathcal{O}^{\star}(M)$, if it satisfies the following equivalent conditions:

(i) $\Gamma$ is a rhombus, generated by some locally separated from zero function $\varphi: M \rightarrow(0 ; 1]$,

$$
\Gamma=\varphi
$$

(ii) $\Gamma$ is a rhombus, generated by some absolutely convex neighborhood of zero $\Delta \subseteq \mathcal{O}^{\star}(M)$, $1 \in{ }^{\circ} \Delta$

$$
\Gamma=\Delta^{\diamond}
$$

(iii) the rhombus generated by $\Gamma$, coincides with $\Gamma$ :

$$
\Gamma=\Gamma^{\diamond}
$$

Proof. The equivalence of conditions (i), (ii), (iii) is proved in the same way as in the case of rectangles.

\section{Properties of rhombuses:}

(i) Every rhombus $\Delta$ in $\mathcal{O}^{\star}(M)$ is a closed absolutely convex neighborhood of zero in $\mathcal{O}^{\star}(M)$. 
(ii) For any neighborhood of zero $\Delta \subseteq \mathcal{O}^{\star}(M)$ the rhombus $\Delta^{\star}$ is the greatest rhombus in $\mathcal{O}^{\star}(M)$, contained in $\Delta$ :

(a) $\Delta^{\diamond}$ is a rhombus in $\mathcal{O}^{\star}(M)$, contained in $\Delta$ :

$$
\Delta^{\diamond} \subseteq \Delta
$$

(b) if $\Gamma$ is another rhombus in $\mathcal{O}^{\star}(M)$, contained in $\Delta$,

$$
\Gamma \subseteq \Delta
$$

then $\Gamma$ is contained in $\Delta$ :

$$
\Gamma \subseteq \Delta^{\diamond}
$$

(iii) Rhombuses in $\mathcal{O}^{\star}(M)$ form a fundamental system of neighborhoods of zero in $\mathcal{O}^{\star}(M)$ : every neighborhood of zero $\Gamma$ in $\mathcal{O}^{\star}(M)$ contains some rhombus.

By analogy with Theorem 2.1 one can prove

Theorem 2.2. The formulas

$$
\Delta=\varphi^{\diamond}, \quad \varphi=\Delta^{\diamond}
$$

establish a bijection between inner envelopes $\varphi$ on $M$ and rhombuses $\Delta$ in $\mathcal{O}^{\star}(M)$.

\section{(e) Duality between rectangles and rhombuses}

Lemma 2.1 implies

Theorem 2.3. The following equalities hold

$$
\begin{aligned}
\left(f^{\mathbf{\bullet}}\right)^{\circ} & =\left(\frac{1}{f}\right)^{\diamond}, & \circ\left(\varphi^{\diamond}\right) & =\left(\frac{1}{\varphi}\right)^{\mathbf{}}, \\
\left(D^{\circ}\right)^{\diamond} & =\frac{1}{D^{\square},} & \left({ }^{\circ} \Delta\right)^{\square} & =\frac{1}{\Delta^{\diamond},} \\
\frac{1}{f^{\square}} & =\left(\frac{1}{f}\right)^{\diamond}, & \frac{1}{\varphi} & =\left(\frac{1}{\varphi}\right)^{\square}, \\
\left(D^{\square}\right)^{\circ} & =\left(D^{\circ}\right)^{\diamond}, & \circ\left(\Delta^{\diamond}\right) & =\left({ }^{\circ} \Delta\right)^{\square},
\end{aligned}
$$

where $f: M \rightarrow[1 ;+\infty)$ is an arbitrary locally bounded function, $\varphi: M \rightarrow(0 ; 1]$ an arbitrary locally separated from zero function, $D$ an arbitrary absolutely convex compact set in $\mathcal{O}(M), \Delta$ an arbitrary closed absolutely convex neighborhood of zero in $\mathcal{O}^{\star}(M)$.

Proof. 1. The first formula in (2.28) follows from (2.14):

$$
\left(f^{\mathbf{\omega}}\right)^{\circ}=(2.14)=\overline{\operatorname{absconv}}\left\{\frac{1}{f(x)} \cdot \delta^{x} ; \quad x \in M\right\}=(2.18)=\left(\frac{1}{f}\right)
$$

After that the substitution $f=\frac{1}{\varphi}$ gives the second formula:

$$
\left(\frac{1}{\varphi}\right)^{\mathbf{0}}=\varphi^{\diamond} \quad \Longrightarrow \quad\left(\frac{1}{\varphi}\right)^{\mathbf{|}}=\circ\left(\left(\frac{1}{\varphi}\right)^{\circ}\right)={ }^{\circ}\left(\varphi^{\bullet}\right)
$$


2. The first formula in (2.29) follows from (2.12):

$$
\frac{1}{D^{\square}(x)}=(\underline{2.12})=\max \left\{\lambda>0: \lambda \cdot \delta^{x} \in D^{\circ}\right\}=(\underline{2.19})=\left(D^{\circ}\right)^{\diamond}(x) \quad \Longrightarrow \quad\left(D^{\circ}\right)^{\diamond}=\frac{1}{D^{\square}}
$$

Then the substitution $D={ }^{\circ} \Delta$ gives the second formula:

$$
D^{\square}=\frac{1}{\left(D^{\circ}\right)^{\diamond}} \quad \Longrightarrow \quad\left({ }^{\circ} \Delta\right)^{\square}=\frac{1}{\left(\left({ }^{\circ} \Delta\right)^{\circ}\right)^{\diamond}}=\frac{1}{\Delta^{\diamond}}
$$

3. Now the first formula in (2.30) follows from the first formula in (2.29) and from the first formula in (2.28) by substitution $D=f^{\mathbf{n}}$ :

$$
\frac{1}{D^{\square}}=\left(D^{\circ}\right)^{\diamond} \quad \Longrightarrow \quad \frac{1}{f \square}=\left(f^{\mathbf{\bullet} \circ}\right)^{\diamond}=\left(\underline{(2.28)}=\left(\frac{1}{f}\right)^{\diamond}\right.
$$

The second formula in (2.30) follows from the second formula in (2.29) and the second formula in (2.28) after the substitution $\Delta=\varphi^{\star}$ :

$$
\frac{1}{\Delta^{\diamond}}=\left({ }^{\circ} \Delta\right)^{\square} \quad \Longrightarrow \quad \frac{1}{\varphi^{\diamond}}=\left({ }^{\circ}\left(\varphi^{\diamond}\right)\right)^{\square}=(\underline{2.28})=\left(\frac{1}{\varphi}\right)^{\square}
$$

4. The first formula in (2.31) follows from the first formula in (2.29) and from the first formula in (2.28):

$$
\left(D^{\circ}\right)^{\diamond}=\frac{1}{D^{\square}} \quad \Longrightarrow \quad\left(D^{\circ}\right)^{\diamond}=\left(\frac{1}{D^{\square}}\right)^{\diamond}=(\underline{2.28})=\left(D^{\square}\right)^{\circ}
$$

Finally the second formula in (2.31) follows from the second formula in (2.29) and from the second formula in (2.28):

$$
\left({ }^{\circ} \Delta\right)^{\square}=\frac{1}{\Delta^{\diamond}} \quad \Longrightarrow \quad\left({ }^{\circ} \Delta\right)^{\square \mathbf{M}}=\left(\frac{1}{\Delta^{\diamond}}\right)^{\mathbf{\bullet}}=(\underline{2.28})={ }^{\circ}\left(\Delta^{\diamond}\right)
$$

Theorem 2.3 implies two important propositions.

Theorem 2.4. The passage to the inverse function

$$
f=\frac{1}{\varphi}, \quad \varphi=\frac{1}{f}
$$

establish a bijection between the outer envelopes $f$ and the inner envelopes $\varphi$ on $M$.

Proof. If $\varphi$ is an inner envelope, then $\varphi=\varphi$, hence $\left(\frac{1}{\varphi}\right)^{-(2.30)}=\frac{1}{\varphi}=\frac{1}{\varphi}$, i.e. $\frac{1}{\varphi}$ is an outer envelope. On the contrary, if $f$ is an outer envelope, then $f \square=f$, therefore $\left(\frac{1}{f}\right)^{\bullet}=(\underline{2.30})=\frac{1}{f}=\frac{1}{f}$, i.e. $\frac{1}{f}$ is an inner envelope.

Theorem 2.5. The passage to polar

$$
D={ }^{\circ} \Delta, \quad \Delta=D^{\circ}
$$

establishes a bijection between rectangles $D$ in $\mathcal{O}(M)$ and rhombuses $\Delta$ in $\mathcal{O}^{\star}(M)$.

Proof. If $\Delta$ is a rhombus, then $\Delta^{\bullet}=\Delta$, hence $\left({ }^{\circ} \Delta\right)^{\square}=(2.31)={ }^{\circ}\left(\Delta^{\diamond}\right)={ }^{\circ} \Delta$, i.e. ${ }^{\circ} \Delta$ is a rectangle. On the contrary, if $D$ is a rectangle, then $D^{\square}=D$, hence $\left(D^{\circ}\right)^{\circ}=(2.31)=\left(D^{\circ}\right)^{\circ}=D^{\circ}$, i.e. $D^{\circ}$ is a rhombus. Since the passage to polar is a bijection between closed absolutely convex sets, it is a bijection between rectangles and rhombuses. 
Theorems 2.4, 2.5 and 2.3 imply

Theorem 2.6. The following equalities hold:

$$
\begin{aligned}
& \left(\left(f^{\mathbf{\square}}\right)^{\circ}\right)^{\diamond}=\frac{1}{f}, \\
& \left({ }^{\circ}\left(\varphi^{\natural}\right)=\frac{1}{\varphi}\right. \\
& \left(\frac{1}{\left(D^{\circ}\right)^{\diamond}}\right)^{\mathbf{\Perp}}=D \\
& \left(\frac{1}{\left({ }^{\circ} \Delta\right)^{\square}}\right)^{\bullet}=\Delta .
\end{aligned}
$$

where $f: M \rightarrow[1 ;+\infty)$ is an arbitrary outer envelope, $\varphi: M \rightarrow(0 ; 1]$ an arbitrary inner envelope, $D$ an arbitrary rectangle in $\mathcal{O}(M), \Delta$ an arbitrary rhombus in $\mathcal{O}^{\star}(M)$.

Proof. If $f$ is an outer envelope, then by Theorem 2.4, $\frac{1}{f}$ is an inner envelope, so

$$
\left(\left(f^{\mathbf{\bullet}}\right)^{\circ}\right)^{\diamond}=(2.28)=\left(\frac{1}{f}\right)^{\diamond}=\frac{1}{f}
$$

The other formulas are proved by analogy.

\section{$\S 3$ Stein groups and Hopf algebras connected to them}

\section{(a) Stein groups, linear groups and algebraic groups}

A complex Lie group $G$ is called a Stein group, if $G$ is a Stein manifold [14. By the Matsushima-Morimoto Theorem [24, XIII.5.9], for complex groups this is equivalent to the condition of holomorphic separability we mentioned at the page 38 .

$$
\forall x \neq y \in G \quad \exists u \in \mathcal{O}(G) \quad u(x) \neq u(y)
$$

Dimension of a Stein group is its dimension as a complex manifold.

Special cases of Stein groups are linear complex groups. They are defined as complex Lie groups which can be represented as closed complex Lie subgroups in the general linear group $\mathrm{GL}_{n}(\mathbb{C})$. In other words, a complex group $G$ is linear, if it is isomorphic to some closed complex subgroup $H$ in $\mathrm{GL}_{n}(\mathbb{C})$ (i.e. there is an isomorphism of groups $\varphi: G \rightarrow H$ which is at the same time a biholomorphic mapping).

Even more narrow class are complex affine algebraic groups. These are subgroups $H$ in $\mathrm{GL}_{n}(\mathbb{C})$, which are at the same time algebraic submanifolds. This means that the group $H$ must be a common set of zeroes for some finite set of polynomials $u_{1}, \ldots, u_{k}$ on $\mathrm{GL}_{n}(\mathbb{C})$ (by polynomial here we can understand a polynomial of matrix elements):

$$
H=\left\{x \in \mathrm{GL}_{n}(\mathbb{C}): u_{1}(x)=\ldots=u_{k}(x)=0\right\}
$$

If a complex group $G$ is isomorphic to some algebraic group $H$ (i.e. there exists an isomorphism of groups $\varphi: G \rightarrow H$ which is at the same time a biholomorphic mapping), then $G$ is also considered an algebraic group, since the algebraic operations on $G$ are regular mappings with respect to the structure of algebraic manifold inherited from $H$.

A Stein group $G$ is called compactly generated, if it has a generating compact set, i.e. a compact set $K \subseteq G$ such that

$$
G=\bigcup_{n \in \mathbb{N}} K^{n}, \quad K^{n}=\underbrace{K \cdot \ldots \cdot K}_{n \text { factors }}
$$

Let us note some examples.

Example 3.1. Complex torus we have mentioned in $\S 2$ i.e. the quotient group $\mathbb{C} /(\mathbb{Z}+i \mathbb{Z})$, is an example of a complex group, which is not a Stein group. 
Example 3.2. Every discrete group $G$ is a Stein group (of zero dimension). Compact sets in $G$ are nothing more than finite sets, hence $G$ will be compactly generated if and only if it is finitely generated. Thus, say, a free group with infinite set of generators can be considered as an example a Stein group which is not compactly generated.

Example 3.3. A discrete group $G$ is algebraic if and only if it is finite. In this case it can be represented as a group of transformations of the space $\mathbb{C}^{n}$, where $n=\operatorname{card} G$ is the number of elements of $G$. For this $\mathbb{C}^{n}$ must be represented as the space of functions from $G$ into $\mathbb{C}$ :

$$
x \in \mathbb{C}^{G} \Longleftrightarrow x: G \rightarrow \mathbb{C},
$$

Then the imbedding of $G$ into $\mathrm{GL}\left(\mathbb{C}^{G}\right)$ (the group of a nondegenerate linear transformations of the space $\left.\mathbb{C}^{G}\right)$ is defined by the formula

$$
\varphi: G \rightarrow \mathrm{GL}\left(\mathbb{C}^{G}\right): \quad \varphi(g)(x)(h)=x(h \cdot g), \quad g, h \in G, \quad x \in \mathbb{C}^{G}
$$

Example 3.4. The general linear group $\mathrm{GL}_{n}(\mathbb{C})$, i.e. the group of a nondegenerate linear transformations of the space $\mathbb{C}^{n}$, is an algebraic group (of dimension $n^{2}$ ). Certainly, $\mathrm{GL}_{n}(\mathbb{C})$ is compactly generated. As a corollary, every linear group is compactly generated.

Example 3.5. The additive group of complex numbers $\mathbb{C}$ is a complex algebraic group (of dimension 1), since it can be embedded into $\mathrm{GL}(2, \mathbb{C})$ by formula

$$
\varphi(\lambda)=\left(\begin{array}{ll}
1 & \lambda \\
0 & 1
\end{array}\right), \quad \lambda \in \mathbb{C}
$$

Example 3.6. The additive group $\mathbb{Z}$ of integers is a complex linear group (of dimension 0), since it can be embedded into $G L(2, \mathbb{C})$ by the same formula

$$
\varphi(n)=\left(\begin{array}{cc}
1 & n \\
0 & 1
\end{array}\right), \quad n \in \mathbb{Z}
$$

But the difference with $\mathbb{C}$ is that $\mathbb{Z}$ is not algebraic: neither in this embedding into $\mathrm{GL}_{n}(\mathbb{C})$, nor in any other one, $\mathbb{Z}$ is a common set for a family of polynomials (this is a result of the fact that $\mathbb{Z}$ is discrete and infinite).

Example 3.7. A multiplicative group $\mathbb{C}^{\times}$of nonzero complex numbers

$$
\mathbb{C}^{\times}=\mathbb{C} \backslash\{0\}
$$

can be represented as general linear group (of nondegenerate transformations of the space $\mathbb{C}$ ),

$$
\mathbb{C}^{\times} \cong \mathrm{GL}_{1}(\mathbb{C})
$$

Thus $\mathbb{C}^{\times}$is a complex algebraic group (of dimension 1). We call this group complex circle.

Example 3.8. Consider the action of group $\mathbb{C}$ on itself by exponents:

$$
\varphi: \mathbb{C} \rightarrow \operatorname{Aut}(\mathbb{C}), \quad \varphi(a)(x)=x \cdot e^{a}
$$

The semidirect product of $\mathbb{C}$ and $\mathbb{C}$ with respect to this action, i.e. a group $\mathbb{C} \ltimes \mathbb{C}$, coinciding with the Cartesian product $\mathbb{C} \times \mathbb{C}$, but endowed with a more complicated multiplication

$$
(a, x) \cdot(b, y):=\left(a+b, x \cdot e^{b}+y\right)
$$

is a (connected) linear complex group, since it can be embedded into $\mathrm{GL}_{3}(\mathbb{C})$ by the homomorphism

$$
(x, a) \mapsto\left(\begin{array}{ccc}
e^{a} & 0 & 0 \\
x & 1 & 0 \\
0 & 0 & e^{i a}
\end{array}\right)
$$

But $\mathbb{C} \ltimes \mathbb{C}$ is not algebraic, since its center

$$
Z(\mathbb{C} \ltimes \mathbb{C})=\{(2 \pi i n, 0) ; n \in \mathbb{Z}\}
$$

is an infinite discrete subgroup (this does not happen with algebraic groups). 


\section{(b) Hopf algebras $\mathcal{O}(G), \mathcal{O}^{\star}(G), \mathcal{R}(G), \mathcal{R}^{\star}(G)$}

The Hopf algebras $\mathbb{C}^{G}$ and $\mathbb{C}_{G}$ we were talking about in $\S 1$ (c) are interesting not so in themselves but more as guiding examples for various similar constructions arising in situations when the initial group $G$ is endowed with some supplementary structure and functions on $G$ preserve this structure. In particular, in [1, examples 10.24-10.27] the author noted standard examples of algebras of functions and functionals, which are stereotype Hopf algebras. If we add Hopf algebras $\mathbb{C}^{G}$ and $\mathbb{C}_{G}$ to those examples, we obtain the following list:

\begin{tabular}{|l|l|l|}
\hline class of groups & algebra of functions & algebra of functionals \\
\hline "pure" groups & $\begin{array}{l}\text { algebra } \mathbb{C}^{G} \\
\text { of all functions on } G\end{array}$ & $\begin{array}{l}\text { algebra } \mathbb{C}_{G} \\
\text { of point charges on } G\end{array}$ \\
\hline algebraic groups & $\begin{array}{l}\text { algebra } \mathcal{R}(G) \\
\text { of polynomials on } G\end{array}$ & $\begin{array}{l}\text { algebra } \mathcal{R}^{\star}(G) \\
\text { of currents of degree } 0 \text { on } G\end{array}$ \\
\hline Stein groups & $\begin{array}{l}\text { algebra } \mathcal{O}(G) \\
\text { of holomorphic functions on } G\end{array}$ & $\begin{array}{l}\text { algebra } \mathcal{O}^{\star}(G) \\
\text { of analytical } \\
\text { functionals on } G\end{array}$ \\
\hline Lie groups & $\begin{array}{l}\text { algebra } \mathcal{E}(G) \\
\text { of smooth functions on } G\end{array}$ & $\begin{array}{l}\text { algebra } \mathcal{E}^{\star}(G) \\
\text { of distributions on } G\end{array}$ \\
\hline locally compact groups & $\begin{array}{l}\text { algebra } \mathcal{C}(G) \\
\text { of continuous functions on } G\end{array}$ & $\begin{array}{l}\text { algebra } \mathcal{C}^{\star}(G) \\
\text { of Radon measures on } G\end{array}$ \\
\hline
\end{tabular}

In [1] the last four examples were mentioned without proof, so we see fit to explain here why those algebras are indeed stereotype Hopf algebras. Stein groups and algebraic groups will be case studies for us.

Hopf algebras $\mathcal{O}(G)$ and $\mathcal{O}^{\star}(G)$ on a Stein group $G$. If $G$ is a Stein group, then the proposition that algebra $\mathcal{O}(G)$ of holomorphic functions on $G$ (with the usual topology of uniform convergence on compact sets in $G$ ) is an injective stereotype Hopf algebra, is proved exactly like this was done for $\mathbb{C}^{G}$. A significant aspect in those reasonings is the isomorphism of functors connecting Cartesian product of groups $\times$ with the corresponding tensor products of functional spaces $\odot$ and $\circledast$ 1, Theorem 8.13],

$$
\mathcal{O}(G \times H) \stackrel{\rho_{G, H}}{\cong} \mathcal{O}(G) \odot \mathcal{O}(H) \stackrel{@}{\cong} \mathcal{O}(G) \circledast \mathcal{O}(H)
$$

- here $\rho_{G, H}$ is defined by identity analogous to (1.25):

$$
\rho_{G, H}(u \boxminus v)=u \odot v, \quad u \in \mathcal{O}(G), \quad v \in \mathcal{O}(H)
$$

and the function $u \boxminus v$ is again defined by formula (1.24).

After defining $\rho_{G, H}$, the multiplication and comultiplication in $\mathcal{O}(G)$ are defined initially on (or with values in) the space $\mathcal{O}(G \times G)$ of functions on the Cartesian square $G \times G$, and then the passage to tensor square is carried out with the help of the isomorphism $\rho_{G, G}$ :

$$
\begin{array}{rll}
\text { multiplication: } & \mu=\widetilde{\mu} \circ \rho_{G, G}: \mathcal{O}(G) \odot \mathcal{O}(G) \rightarrow \mathcal{O}(G), & \widetilde{\mu}(v)(t)=v(t, t) \\
\text { unit: } & \iota: \mathbb{C} \rightarrow \mathcal{O}(G), & \iota(\lambda)(t)=\lambda \\
\text { comultiplication: } & \varkappa=\rho_{G, G} \circ \tilde{\varkappa}: \mathcal{O}(G) \rightarrow \mathcal{O}(G) \odot \mathcal{O}(G), & \widetilde{\varkappa}(u)(s, t)=u(s \cdot t) \\
\text { counit: } & \varepsilon: \mathcal{O}(G) \rightarrow \mathbb{C}, & \varepsilon(u)=u\left(1_{G}\right) \\
\text { antipode: } & \sigma: \mathcal{O}(G) \rightarrow \mathcal{O}(G), & \sigma(u)(t)=u\left(t^{-1}\right)
\end{array}
$$


This can be illustrated by the following picture:

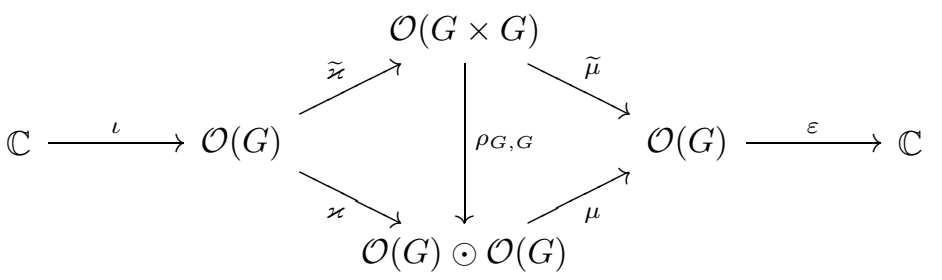

The fact that this defines an injective stereotype Hopf algebra is proved literally like Theorem 1.7, And again, like in the case of $\mathbb{C}^{G}$, the Hopf algebra $\mathcal{O}(G)$ becomes a projective Hopf algebra (hence, a rigid Hopf algebra in the sense of definition $\S 1[(b)]$, because of the second equality in (3.1).

Theorem 3.1. For any Stein group $G$

- the algebra $\mathcal{O}(G)$ of holomorphic functions on $G$ is a rigid stereotype Hopf algebra with respect to algebraic operations, defined by formulas (3.3)-(3.7);

- its dual algebra $\mathcal{O}^{\star}(G)$ of analytical functionals on $G$ is a rigid stereotype Hopf algebra with respect to the dual algebraic operations.

If in addition the group $G$ is compactly generated, then $\mathcal{O}(G)$ is a nuclear Hopf-Fréchet algebra, and $\mathcal{O}^{\star}(G)$ a nuclear Hopf-Brauner algebra.

Hopf algebras $\mathcal{R}(G)$ and $\mathcal{R}^{\star}(G)$ on an affine algebraic group $G$. Let $G$ be an affine algebraic group, $\mathcal{R}(G)$ the algebra of polynomials on $G$ (with the strongest locally convex topology). Like in the previous cases, the identity

$$
\rho_{G, H}(u \boxminus v)=u \odot v, \quad u \in \mathcal{R}(G), \quad v \in \mathcal{R}(H)
$$

(again, $u \boxminus v$ is defined by formula (1.24) ) defines an isomorphism of functors, connecting the Cartesian product of groups $\times$ and tensor products of functional spaces $\odot$ and $\circledast$ [1, Theorem 8.16]:

$$
\mathcal{R}(G \times H) \stackrel{\rho_{G, H}}{\cong} \mathcal{R}(G) \odot \mathcal{R}(H) \stackrel{\Theta^{-1}}{\cong} \mathcal{R}(G) \circledast \mathcal{R}(H)
$$

Those isomorphisms then define algebraic operations on $\mathcal{R}(G)$ by formulas, analogous to (3.3)-(3.7). As a result we come to the following

Theorem 3.2. For any affine algebraic group $G$

- the algebra $\mathcal{R}(G)$ of polynoimals on $G$ is a rigid stereotype Hopf-Brauner algebra;

- the dual algebra $\mathcal{R}^{\star}(G)$ of currents of degree 0 on $G$ is an injective stereotype Hopf-Fréchet algebra.

Convolutions in $\mathcal{R}^{\star}(G)$ and $\mathcal{O}^{\star}(G)$. For some further calculations it is useful to record the formulas defining convolution in the algebras of functionals $\mathcal{R}^{\star}(G)$ and $\mathcal{O}^{\star}(G)$. This definition is anticipated by formulas for shift, antipode and convolution between a functional and a function:

$$
\begin{aligned}
(u \cdot a)(x) & :=u(a \cdot x), & (a \cdot u)(x) & :=u(x \cdot a), & & u \in \mathcal{O}(G), a, x \in G \\
(\alpha \cdot a)(u) & :=\alpha(a \cdot u), & (a \cdot \alpha)(u) & :=\alpha(u \cdot a), & & \alpha \in \mathcal{O}^{\star}(G), u \in \mathcal{O}(G), a \in G \\
\tilde{u}(x) & :=u\left(x^{-1}\right), & \tilde{\alpha}(u) & :=\alpha(\tilde{u}), & & \alpha \in \mathcal{O}^{\star}(G), u \in \mathcal{O}(G), x \in G \\
(\alpha * u)(x) & :=\alpha(\widetilde{x \cdot u}), & (u * \alpha)(x) & :=\alpha(\widetilde{u \cdot x}) & \alpha & \in \mathcal{O}^{\star}(G), u \in \mathcal{O}(G), x \in G
\end{aligned}
$$

Then the convolution of functionals is defined by formula

$$
\alpha * \beta(u):=\alpha(\widetilde{\beta * \tilde{u}})=\tilde{\alpha}(\beta * \tilde{u})=\beta(\tilde{\alpha} * u) \quad \alpha, \beta \in \mathcal{O}^{\star}(G), u \in \mathcal{O}(G)
$$

In particular, the convolution with the delta-functional is a shift:

$$
\delta^{a} * \beta=a \cdot \beta \quad \beta * \delta^{a}=\beta \cdot a \quad \beta \in \mathcal{O}^{\star}(G), a \in G
$$




\section{(c) Examples}

It is useful to illustrate the latter theorems by several examples. For this we choose the main examples of Abelian Stein groups $-\mathbb{Z}, \mathbb{C}^{\times}, \mathbb{C}$ - apart from everything else, these examples will be useful below in $\S 6$ and $\$ 7$.

Algebras $\mathcal{O}(\mathbb{Z})$ and $\mathcal{O}^{\star}(\mathbb{Z})$. We have already noted in Example 3.6 that the group $\mathbb{Z}$ of integers can be considered as a complex group (of dimension 0 ). Since $\mathbb{Z}$ is discrete, each function on $\mathbb{Z}$ is automatically holomorphic, so the algebra $\mathcal{O}(\mathbb{Z})$ formally coincides with the algebra $\mathbb{C}^{\mathbb{Z}}$, and the algebra $\mathcal{O}^{\star}(\mathbb{Z})$ with the algebra $\mathbb{C}_{\mathbb{Z}}$ :

$$
\mathcal{O}(\mathbb{Z})=\mathbb{C}^{\mathbb{Z}}, \quad \mathcal{O}^{\star}(\mathbb{Z})=\mathbb{C}_{\mathbb{Z}}
$$

As a corollary the structure of these algebras is described by formulas (1.19)-(1.23): the characteristic functions of singletons

$$
1_{n}(m)=\left\{\begin{array}{ll}
0, & m=n \\
1, & m=n
\end{array}, \quad m \in \mathbb{Z}, n \in \mathbb{Z}\right.
$$

form a basis in the stereotype space $\mathcal{O}(\mathbb{Z})=\mathbb{C}^{\mathbb{Z}}$, and delta-functionals

$$
\delta^{k}(u)=u(k), \quad u \in \mathcal{O}(\mathbb{Z})
$$

a dual (algebraic) basis in $\mathcal{O}^{\star}(\mathbb{Z})=\mathbb{C}_{\mathbb{Z}}$ :

$$
\left\langle 1_{n}, \delta^{k}\right\rangle= \begin{cases}0, & n \neq k \\ 1, & n=k\end{cases}
$$

It is convenient to represent elements of $\mathcal{O}(\mathbb{Z})$ and $\mathcal{O}^{\star}(\mathbb{Z})$ in the form of series (which converge in these spaces)

$$
\begin{array}{llll}
u \in \mathcal{O}(\mathbb{Z})=\mathbb{C}^{\mathbb{Z}} & \Longleftrightarrow & u=\sum_{n \in \mathbb{Z}} u(n) \cdot 1_{n}, & u(n)=\delta^{n}(u), \\
\alpha \in \mathcal{O}^{\star}(\mathbb{Z})=\mathbb{C}_{\mathbb{Z}} & \Longleftrightarrow & \alpha=\sum_{n \in \mathbb{Z}} \alpha_{n} \cdot \delta^{n}, & \alpha_{n}=\alpha\left(1_{n}\right),
\end{array}
$$

where the action of $\alpha$ on $u$ is described by formula

$$
\langle u, \alpha\rangle=\sum_{n \in \mathbb{Z}} u(n) \cdot \alpha_{n}
$$

The operations of multiplication in $\mathcal{O}(\mathbb{Z})=\mathbb{C}^{\mathbb{Z}}$ and in $\mathcal{O}^{\star}(\mathbb{Z})=\mathbb{C}_{\mathbb{Z}}$ are represented by series:

$$
u \cdot v=\sum_{n \in \mathbb{Z}} u(n) \cdot v(n) \cdot 1_{n}, \quad \alpha * \beta=\sum_{k \in \mathbb{Z}}\left(\sum_{i \in \mathbb{Z}} \alpha_{i} \cdot \beta_{k-i}\right) \cdot \delta^{k},
$$

(in the first case this is the coordinate-wise multiplication, and in the second case the multiplication of power series).

Proposition 3.1. The algebra $\mathcal{O}(\mathbb{Z})=\mathbb{C}^{\mathbb{Z}}$ of functions on $\mathbb{Z}$ is a nuclear Hopf-Fréchet algebra with the topology generated by seminorms

$$
\|u\|_{N}=\sum_{|n| \leqslant N}|u(n)|, \quad N \in \mathbb{N}
$$


and with algebraic operations defined on basis elements $1_{k}$ by formulas

$$
\begin{array}{ll}
1_{m} \cdot 1_{n}= \begin{cases}1_{m}, & m=n \\
0, & m \neq n\end{cases} & 1_{\mathcal{R}^{\star}\left(\mathbb{C}^{\times}\right)}=\sum_{n \in \mathbb{Z}} 1_{n} \\
\varkappa\left(1_{n}\right)=\sum_{m \in \mathbb{Z}} 1_{m} \odot 1_{n-m} & \varepsilon\left(1_{n}\right)= \begin{cases}1, & n=0 \\
0, & n \neq 0\end{cases} \\
\sigma\left(1_{n}\right)=1_{-n} &
\end{array}
$$

Proposition 3.2. The algebra $\mathcal{O}^{\star}(\mathbb{Z})=\mathbb{C}_{\mathbb{Z}}$ of point charges on $\mathbb{Z}$ is a nuclear Hopf-Brauner algebra with the topology generated by seminorms

$$
\|\left.|\alpha|\right|_{r}=\sum_{n \in \mathbb{Z}} r_{n} \cdot\left|\alpha_{n}\right| \quad\left(r_{n} \geqslant 0\right)
$$

and algebraic operations defined on basis monomials $\delta^{k}$ by formulas

$$
\begin{aligned}
& \delta^{k} * \delta^{l}=\delta^{k+l} \\
& \varkappa\left(\delta^{k}\right)=\delta^{k} \circledast \delta^{k} \\
& 1_{\mathcal{R}\left(\mathbb{C}^{\times}\right)}=\delta^{0} \\
& \sigma\left(\delta^{k}\right)=\delta^{-k}
\end{aligned}
$$

Proof. It can be not obvious here that seminorms (3.24) indeed define the topology in $\mathcal{O}^{\star}(\mathbb{Z})=\mathbb{C}_{\mathbb{Z}}$. The auxiliary statement used here will be also useful for us below in Lemma 6.3, so we formulate it separately:

Lemma 3.1. If $p$ is a continuous seminorm on $\mathcal{O}^{\star}(\mathbb{Z})$, and $r_{n}=p\left(\delta^{n}\right)$, then $p$ is majorized by seminorm (3.24):

$$
p(\alpha) \leqslant\||| \alpha\|_{r}
$$

Proof.

$$
p(\alpha)=p\left(\sum_{n \in \mathbb{Z}} \alpha_{n} \cdot \delta^{n}\right) \leqslant \sum_{n \in \mathbb{Z}}\left|\alpha_{n}\right| \cdot p\left(\delta^{n}\right)=\sum_{n \in \mathbb{Z}}\left|\alpha_{n}\right| \cdot r_{n}=|||\alpha| \|_{r}
$$

Algebras $\mathcal{R}\left(\mathbb{C}^{\times}\right), \mathcal{R}^{\star}\left(\mathbb{C}^{\times}\right), \mathcal{O}\left(\mathbb{C}^{\times}\right), \mathcal{O}^{\star}\left(\mathbb{C}^{\times}\right)$. In Example 3.7 we denoted by $\mathbb{C}^{\times}$the multiplicative group of nonzero complex numbers:

$$
\mathbb{C}^{\times}:=\mathbb{C} \backslash\{0\} .
$$

(the multiplication in $\mathbb{C}^{\times}$is the usual multiplication of complex numbers). We call this group complex circle.

The algebra $\mathcal{R}\left(\mathbb{C}^{\times}\right)$of polynomials on $\mathbb{C}^{\times}$consists of Laurent polynomials, i.e. of function of the form

$$
u=\sum_{n \in \mathbb{Z}} u_{n} \cdot z^{n}
$$

where $z^{n}$ are monomials on $\mathbb{C}^{\times}$:

$$
z^{n}(x):=x^{n}, \quad x \in \mathbb{C}^{\times}, \quad n \in \mathbb{Z}
$$

and almost all $u_{n} \in \mathbb{C}$ vanish:

$$
\operatorname{card}\left\{n \in \mathbb{Z}: u_{n} \neq 0\right\}<\infty
$$


And the algebra $\mathcal{R}^{\star}\left(\mathbb{C}^{\times}\right)$of currents on $\mathbb{C}^{\times}$consists of functionals

$$
\alpha=\sum_{k \in \mathbb{Z}} \alpha_{k} \cdot \zeta^{k}
$$

where $\zeta_{k}$ is the functional of finding the $k$-th Laurent coefficient:

$$
\zeta_{k}(u)=\frac{1}{2 \pi i} \int_{|z|=1} \frac{u(z)}{z^{k+1}} \mathrm{~d} z=\int_{0}^{1} e^{-2 \pi i k t} u\left(e^{2 \pi i t}\right) \mathrm{d} t, \quad u \in \mathcal{R}\left(\mathbb{C}^{\times}\right)
$$

and $\alpha_{k} \in \mathbb{C}$ is an arbitrary sequence.

Monomials $\zeta_{k}$ and $z^{n}$ act on each other by formula

$$
\left\langle z^{n}, \zeta_{k}\right\rangle= \begin{cases}1, & n=k \\ 0, & n \neq k\end{cases}
$$

so the action of a current $\alpha$ on a polynomial $u$ is described by formula

$$
\langle u, \alpha\rangle=\sum_{n \in \mathbb{Z}} u_{n} \cdot \alpha_{n}
$$

and the operations of multiplication in $\mathcal{R}\left(\mathbb{C}^{\times}\right)$and in $\mathcal{R}^{\star}\left(\mathbb{C}^{\times}\right)$are represented by series as follows:

$$
u \cdot v=\sum_{n \in \mathbb{Z}}\left(\sum_{i \in \mathbb{Z}} u_{i} \cdot v_{n-i}\right) \cdot z^{n}, \quad \alpha * \beta=\sum_{k \in \mathbb{Z}} \alpha_{k} \cdot \beta_{k} \cdot \zeta_{k},
$$

(in the first case this is the multiplication of series, and in the second case the coordinate-wise multiplication).

Proposition 3.3. The mapping

$$
u \in \mathcal{R}\left(\mathbb{C}^{\times}\right) \mapsto\left\{u_{k} ; k \in \mathbb{Z}\right\} \in \mathbb{C}_{\mathbb{Z}}
$$

is an isomorphism of nuclear Hopf-Brauner algebras

$$
\mathcal{R}\left(\mathbb{C}^{\times}\right) \cong \mathbb{C}_{\mathbb{Z}}
$$

Proposition 3.4. The algebra $\mathcal{R}\left(\mathbb{C}^{\times}\right)$of polynomials on the complex circle $\mathbb{C}^{\times}$is a nuclear Hopf-Fréchet algebra with the topology generated by seminorms

$$
\left\|\left|u \|_{r}=\sum_{n \in \mathbb{Z}} r_{n} \cdot\right| u_{n} \mid \quad\left(r_{n} \geqslant 0\right)\right.
$$

and algebraic operations defined on monomials $z^{k}$ by formulas

$$
\begin{array}{rlrl}
z^{k} \cdot z^{l}=z^{k+l} & & 1_{\mathcal{R}\left(\mathbb{C}^{\times}\right)}=z^{0} \\
\varkappa\left(z^{k}\right) & =z^{k} \odot z^{k} & & \varepsilon\left(z^{k}\right)=1 \\
\sigma\left(z^{k}\right) & =z^{-k} &
\end{array}
$$

Proposition 3.5. The mapping

$$
\alpha \in \mathcal{R}^{\star}\left(\mathbb{C}^{\times}\right) \mapsto\left\{\alpha_{k} ; k \in \mathbb{Z}\right\} \in \mathbb{C}^{\mathbb{Z}}
$$

is an isomorphism of nuclear Hopf-Fréchet algebras

$$
\mathcal{R}^{\star}\left(\mathbb{C}^{\times}\right) \cong \mathbb{C}^{\mathbb{Z}}
$$


Proposition 3.6. The algebra $\mathcal{R}^{\star}\left(\mathbb{C}^{\times}\right)$of currents on the complex circle $\mathbb{C}^{\times}$is a nuclear Hopf-Fréchet algebra with the topology generated by seminorms

$$
\|\alpha\|_{N}=\sum_{|n| \leqslant N}\left|\alpha_{n}\right| \quad(N \in \mathbb{N})
$$

and algebraic operations defined on basis elements $\zeta_{k}$ by formulas

$$
\begin{aligned}
\zeta_{k} * \zeta_{l}= \begin{cases}\zeta_{l}, & k=l \\
0, & k \neq l\end{cases} & 1_{\mathcal{R}^{\star}\left(\mathbb{C}^{\times}\right)}=\sum_{n \in \mathbb{Z}} \zeta_{n} \\
\varkappa\left(\zeta_{k}\right)=\sum_{l \in \mathbb{Z}} \zeta_{l} \circledast \zeta_{k-l} & \varepsilon\left(\zeta_{k}\right)= \begin{cases}1, & k=0 \\
0, & k \neq 0\end{cases} \\
\sigma\left(\zeta_{k}\right)=\zeta_{-k} &
\end{aligned}
$$

As usual, by symbol $\mathcal{O}\left(\mathbb{C}^{\times}\right)$we denote the algebra of holomorphic functions on the complex circle $\mathbb{C}^{\times}$, and by $\mathcal{O}^{\star}\left(\mathbb{C}^{\times}\right)$its dual algebra of analytic functionals on $\mathbb{C}^{\times}$.

It is useful to represent the elements of algebras $\mathcal{O}\left(\mathbb{C}^{\times}\right)$and $\mathcal{O}^{\star}\left(\mathbb{C}^{\times}\right)$by series

$$
\begin{aligned}
& u \in \mathcal{O}\left(\mathbb{C}^{\times}\right) \quad \Longleftrightarrow u=\sum_{n \in \mathbb{Z}} u_{n} \cdot z^{n}, \quad u_{n} \in \mathbb{C}: \quad \forall C>0 \quad \sum_{n \in \mathbb{Z}}\left|u_{n}\right| \cdot C^{|n|}<\infty \quad\left(u_{n}=\zeta_{n}(u)\right) \\
& \alpha \in \mathcal{O}^{\star}\left(\mathbb{C}^{\times}\right) \quad \Longleftrightarrow \quad \alpha=\sum_{n \in \mathbb{Z}} \alpha_{n} \cdot \zeta_{n}, \quad \alpha_{n} \in \mathbb{C}: \quad \exists C>0 \quad \forall n \in \mathbb{N} \quad\left|\alpha_{n}\right| \leqslant C^{|n|} \quad\left(\alpha_{n}=\alpha\left(z^{n}\right)\right)
\end{aligned}
$$

Like in the case of $\mathcal{R}\left(\mathbb{C}^{\times}\right)$and $\mathcal{R}^{\star}\left(\mathbb{C}^{\times}\right)$, the action of $\alpha$ on $u$ is described by formula

$$
\langle u, \alpha\rangle=\sum_{n \in \mathbb{Z}} u_{n} \cdot \alpha_{n}
$$

and the operations of multiplication in $\mathcal{O}\left(\mathbb{C}^{\times}\right)$and $\mathcal{O}^{\star}\left(\mathbb{C}^{\times}\right)$can be written as the usual multiplication of series in the first case, and a coordinate-wise multiplication in the second case:

$$
u \cdot v=\sum_{n \in \mathbb{Z}}\left(\sum_{i \in \mathbb{Z}} u_{i} \cdot v_{n-i}\right) \cdot z^{n}, \quad \alpha * \beta=\sum_{n \in \mathbb{Z}} \alpha_{n} \cdot \beta_{n} \cdot \zeta_{n},
$$

Proposition 3.7. The algebra $\mathcal{O}\left(\mathbb{C}^{\times}\right)$of holomorphic functions on the complex circle $\mathbb{C}^{\times}$is a nuclear Hopf-Fréchet algebra with the topology generated by seminorms

$$
\|u\|_{C}=\sum_{n \in \mathbb{Z}}\left|u_{n}\right| \cdot C^{|n|}, \quad C \geqslant 1 .
$$

and algebraic operations defined on monomials $z^{k}$ by the same formulas (3.36)-(3.38) as in the case of $\mathcal{R}\left(\mathbb{C}^{\times}\right)$:

$$
\begin{aligned}
& z^{k} \cdot z^{l}=z^{k+l} \\
& \varkappa\left(z^{k}\right)=z^{k} \odot z^{k} \\
& \sigma\left(z^{k}\right)=z^{-k} \\
& \begin{array}{l}
1_{\mathcal{O}\left(\mathbb{C}^{\times}\right)}=z^{0} \\
\varepsilon\left(z^{k}\right)=1
\end{array}
\end{aligned}
$$

Proof. Every usual seminorm $|u|_{K}=\max _{x \in K}|u(x)|$, where $K$ is a compact set in $\mathbb{C}^{\times}$, is majorized by some seminorm $\|u\|_{C}$, namely the one with $C=\max _{x \in K} \max \left\{|x|, \frac{1}{|x|}\right\}$ :

$$
|u|_{K}=\max _{x \in K}|u(x)|=\max _{x \in K}\left|\sum_{n \in \mathbb{Z}} u_{n} \cdot x^{n}\right| \leqslant \max _{x \in K} \sum_{n \in \mathbb{Z}}\left|u_{n}\right| \cdot\left|x^{n}\right| \leqslant \sum_{n \in \mathbb{Z}}\left|u_{n}\right| \cdot C^{|n|}=\|u\|_{C}
$$


On the contrary, for every number $C \geqslant 1$ we can take a compact set $K=\left\{t \in \mathbb{C}: \frac{1}{C+1} \leqslant|t| \leqslant C+1\right\}$, and then from the Cauchy formulas for Laurent coefficients

$$
\left|u_{n}\right| \leqslant|u|_{K} \cdot \min \left\{(C+1)^{n} ; \frac{1}{(C+1)^{n}}\right\}=|u|_{K} \cdot(C+1)^{-|n|}=\frac{|u|_{K}}{(C+1)^{|n|}}
$$

we have that $\|u\|_{C}$ is majorized by $|u|_{K}$ (with coefficient $(C+1)^{2}$ ):

$$
\|u\|_{C}=\sum_{n \in \mathbb{Z}}\left|u_{n}\right| \cdot C^{|n|} \leqslant \sum_{n \in \mathbb{Z}} \frac{|u|_{K}}{(C+1)^{|n|}} \cdot C^{|n|} \leqslant|u|_{K} \cdot \sum_{n \in \mathbb{Z}}\left(\frac{C}{C+1}\right)^{|n|}=(C+1)^{2} \cdot|u|_{K}
$$

Proposition 3.8. The algebra $\mathcal{O}^{\star}\left(\mathbb{C}^{\times}\right)$of analytical functionals on the complex circle $\mathbb{C}^{\times}$is a nuclear Hopf-Brauner algebra with the topology generated by seminorms

$$
\left\||| \alpha\left|\|_{r}=\sup _{u \in E_{r}}\right| \alpha(u)\left|=\sum_{n \in \mathbb{Z}} r_{n} \cdot\right| \alpha_{n} \mid \quad\left(r_{n} \geqslant 0: \forall C>0 \quad \sum_{n \in \mathbb{Z}} r_{n} \cdot C^{|n|}<\infty\right)\right.
$$

and algebraic operations defined on basis elements $\zeta_{k}$ by the same formulas (3.41)-(3.43) as in the case of $\mathcal{R}^{\star}\left(\mathbb{C}^{\times}\right)$:

$$
\begin{array}{rlrl}
\zeta_{k} * \zeta_{l} & =\left\{\begin{array}{lll}
\zeta_{l}, & k=l \\
0, & k \neq l
\end{array}\right. & 1_{\mathcal{O}^{\star}\left(\mathbb{C}^{\times}\right)}=\sum_{n \in \mathbb{Z}} \zeta_{n} \\
\varkappa\left(\zeta_{k}\right)=\sum_{l \in \mathbb{Z}} \zeta_{l} \circledast \zeta_{k-l} & \varepsilon\left(\zeta_{k}\right)= \begin{cases}1, & k=0 \\
0, & k \neq 0\end{cases} \\
\sigma\left(\zeta_{k}\right)=\zeta_{-k} &
\end{array}
$$

Proof. For every sequence of nonnegative numbers $r_{n} \geqslant 0$ satisfying the condition

$$
\forall C>0 \quad \sum_{n \in \mathbb{Z}} r_{n} \cdot C^{|n|}<\infty
$$

the set

$$
E_{r}=\left\{u \in \mathcal{O}\left(\mathbb{C}^{\times}\right): \forall n \in \mathbb{Z} \quad\left|u_{n}\right| \leqslant r_{n}\right\}
$$

is compact in $\mathcal{O}\left(\mathbb{C}^{\times}\right)$since it is closed and is contained in the rectangle $f$, where

$$
f(t)=\sum_{n \in \mathbb{Z}} r_{n} \cdot|t|^{n}, \quad t \in \mathbb{C}^{\times}
$$

Hence, $E_{r}$ generates a continuous seminorm $\alpha \mapsto \max _{u \in E_{r}}|\langle u, \alpha\rangle|$ on $\mathcal{O}^{\star}\left(\mathbb{C}^{\times}\right)$. But this is exactly the seminorm from (3.48):

$$
\max _{u \in E_{r}}|\langle u, \alpha\rangle|=\max _{|u| \leqslant r_{n}}\left|\sum_{n \in \mathbb{Z}} u_{n} \cdot \alpha_{n}\right|=\sum_{n \in \mathbb{Z}} r_{n} \cdot\left|\alpha_{n}\right|=|||\alpha| \|_{r}
$$

It remains to verify that seminorms $\||\cdot|\|_{r}$ indeed generate the topology of the space $\mathcal{O}^{\star}\left(\mathbb{C}^{\times}\right)$. This follows from:

Lemma 3.2. If $p$ is a continuous seminorm on $\mathcal{O}^{\star}\left(\mathbb{C}^{\times}\right)$, then the family of numbers

$$
r_{n}=p\left(\zeta_{n}\right)
$$

satisfy condition (3.49), and $p$ is majorized by the seminorm ||$\cdot|\||_{r}$ :

$$
p(\alpha) \leqslant\||| \alpha\|_{r}
$$


Proof. The set

$$
D=\left\{u \in \mathcal{O}\left(\mathbb{C}^{\times}\right): \sup _{\alpha \in \mathcal{O}^{*}\left(\mathbb{C}^{\times}\right): p(\alpha) \leqslant 1}|\alpha(u)| \leqslant 1\right\}
$$

is compact in $\mathcal{O}\left(\mathbb{C}^{\times}\right)$generating the seminorm $p$ :

$$
p(\alpha)=\sup _{u \in D}|\alpha(u)|
$$

Therefore,

$$
r_{n}=p\left(\zeta_{n}\right)=\sup _{u \in D}\left|\zeta_{n}(u)\right|=\sup _{u \in D}\left|u_{n}\right|
$$

For any $C>0$ we have:

$$
\begin{gathered}
\infty>\sup _{u \in D}|| u \|_{C+1}=\sup _{u \in D} \sum_{n \in \mathbb{Z}}\left|u_{n}\right| \cdot(C+1)^{|n|} \geqslant \sup _{u \in D} \sup _{n}\left|u_{n}\right| \cdot(C+1)^{|n|}=\sup _{n}\left(r_{n} \cdot(C+1)^{|n|}\right) \\
\Downarrow \\
\exists M>0 \quad \forall n \in \mathbb{Z} \quad r_{n} \leqslant \frac{M}{(C+1)^{|n|}} \\
\Downarrow \\
\sum_{n \in \mathbb{Z}} r_{n} \cdot C^{|n|} \leqslant \sum_{n \in \mathbb{Z}} \frac{M}{(C+1)^{|n|}} \cdot C^{|n|}<\infty
\end{gathered}
$$

i.e. $r_{n}$ indeed satisfy (3.49). The formula (3.51) is proved by a sequence of inequalities analogous to (3.29).

The chain $\mathcal{R}(\mathbb{C}) \subset \mathcal{O}(\mathbb{C}) \subset \mathcal{O}^{\star}(\mathbb{C}) \subset \mathcal{R}^{\star}(\mathbb{C})$. By symbol $\mathcal{R}(\mathbb{C})$ we denote the usual algebra of polynomials on the complex plane $\mathbb{C}$. Let $t^{k}$ denote the monomial of degree $k \in \mathbb{N}$ on $\mathbb{C}$ :

$$
t^{k}(x):=x^{k}, \quad x \in \mathbb{C}, k \in \mathbb{N}
$$

Every polynomial $u \in \mathcal{R}(\mathbb{C})$ is uniquely represented by the series (with finite number of nonzero terms)

$$
u=\sum_{k \in \mathbb{N}} u_{k} \cdot t^{k}, \quad u_{k} \in \mathbb{C}: \quad \operatorname{card}\left\{k \in \mathbb{N}: u_{k} \neq 0\right\}<\infty
$$

so the monomials $t^{k}$ form an algebraic basis in the space $\mathcal{R}(\mathbb{C})$. The multiplication in $\mathcal{R}(\mathbb{C})$ is the usual multiplication of polynomials

$$
u \cdot v=\sum_{k \in \mathbb{N}}\left(\sum_{i=0}^{n} u_{k-i} \cdot v_{i}\right) \cdot t^{k}
$$

and the topology in $\mathcal{R}(\mathbb{C})$ is defined as the strongest locally convex topology. This implies

Proposition 3.9. The mapping

$$
u \in \mathcal{R}(\mathbb{C}) \mapsto\left\{u_{k} ; k \in \mathbb{N}\right\} \in \mathbb{C}_{\mathbb{N}}
$$

is an isomorphism of topological vector spaces

$$
\mathcal{R}(\mathbb{C}) \cong \mathbb{C}_{\mathbb{N}}
$$


Remark 3.1. We can interpret formula (3.54) as isomorphism of algebras, if the multiplication in $\mathbb{C}_{\mathbb{N}}$ is defined by the same formula (1.23) as for algebra $\mathbb{C}_{G}$ of point charges on the group $G$ (defined in $\left.\S 1(\mathrm{c})\right]$, we should only remember here that the set $\mathbb{N}$ is not a group, but a monoid with respect to the additive operation used for this purpose.

On the other hand formula (3.54) is not an isomorphism of Hopf algebras, in particular, since $\mathbb{C}_{\mathbb{N}}$ is not a Hopf algebra at all with respect to the operations defined in $\S 1$ (c) for $x \in \mathbb{N}$ the inverse element $x^{-1}=-x$ does not exist when $x \neq 0$, so the antipode here cannot be defined by equality (1.27).

Proposition 3.10. The algebra $\mathcal{R}(\mathbb{C})$ of polynomials on the complex plane $\mathbb{C}$ is a nuclear Hopf-Brauner algebra with the topology generated by seminorms

$$
\|u\|_{r}=\sum_{k \in \mathbb{N}} r_{k} \cdot\left|u_{k}\right|, \quad\left(r_{k} \geqslant 0\right)
$$

and algebraic operations defined on monomials $t^{k}$ by formulas

$$
\begin{array}{rlrl}
t^{k} \cdot t^{l} & =t^{k+l} & 1_{\mathcal{R}(\mathbb{C})}=t^{0} \\
\varkappa\left(t^{k}\right)=\sum_{i=0}^{k}\left(\begin{array}{c}
k \\
i
\end{array}\right) \cdot t^{k-i} \odot t^{i} & \varepsilon\left(t^{k}\right)= \begin{cases}1, & k=0 \\
0, & k>0\end{cases} \\
\sigma\left(t^{k}\right)=(-1)^{k} \cdot t^{k} &
\end{array}
$$

Proof. The algebraic operations which we did not find yet - comultiplication, counit and antipode - are computed by formulas (3.5)-(3.7). For instance, comultiplication:

$$
\begin{gathered}
\tilde{\varkappa}\left(t^{k}\right)(x, y)=t^{k}(x+y)=(x+y)^{k}=\sum_{i=0}^{k}\left(\begin{array}{c}
k \\
i
\end{array}\right) \cdot x^{k-i} \cdot y^{i}=\sum_{i=0}^{k}\left(\begin{array}{c}
k \\
i
\end{array}\right) \cdot t^{k-i} \boxminus t^{i}(x, y) \\
\Downarrow \\
\tilde{\varkappa}\left(t^{k}\right)=\sum_{i=0}^{k}\left(\begin{array}{c}
k \\
i
\end{array}\right) \cdot t^{k-i} \boxminus t^{i} \\
\Downarrow \\
\varkappa\left(t^{k}\right)=\rho_{\mathbb{C}, \mathbb{C}}\left(\widetilde{\varkappa}\left(t^{k}\right)\right)=\sum_{i=0}^{k}\left(\begin{array}{c}
k \\
i
\end{array}\right) \cdot t^{k-i} \odot t^{i}
\end{gathered}
$$

Following the terminology of [1], we call a current of degree 0 , or a current on $\mathbb{C}$ an arbitrary linear functional $\alpha: \mathcal{R}(\mathbb{C}) \rightarrow \mathbb{C}$ on the space of polynomials $\mathcal{R}(\mathbb{C})$ (every such a functional is automatically continuous). Formula (3.54) implies that every compact set in the space of polynomials $\mathcal{R}(\mathbb{C})$ is finitedimensional, hence it is contained in a convex hull of a finite set of basis monomials $t^{k}$. Therefore the topology of the space $\mathcal{R}^{\star}(\mathbb{C}$ ) of currents on $\mathbb{C}$ (which is formally defined as the topology of uniform convergence on compact sets in $\mathcal{R}(\mathbb{C})$ ) can be defined as the topology of convergence on monomials $t^{k}$, i.e. the topology generated by seminorms

$$
\|\alpha\|_{N}=\sum_{k \in N}\left|\alpha\left(t^{k}\right)\right|
$$

where $N$ is an arbitrary finite set in $\mathbb{N}$.

The functional of taking derivative of the $k$-th derivative in the point 0 is a typical example of a current:

$$
\tau^{k}(u)=\left.\left(\frac{\mathrm{d}^{k}}{\mathrm{~d} x^{k}} u(x)\right)\right|_{x=0}, \quad u \in \mathcal{R}(\mathbb{C})
$$


By the Taylor theorem, these functionals are connected with the coefficients $u_{k}$ in the decomposition (3.53) of a polynomial $u \in \mathcal{R}(\mathbb{C})$ through the formula

$$
u_{k}=\frac{1}{k !} \cdot \tau^{k}(u)
$$

Therefore the action of a current $\alpha \in \mathcal{R}^{\star}(\mathbb{C})$ on a polynomial $u \in \mathcal{R}(\mathbb{C})$ can be written by formula

$$
\alpha(u)=\alpha\left(\sum_{k \in \mathbb{N}} u_{k} \cdot t^{k}\right)=\sum_{k \in \mathbb{N}} u_{k} \cdot \alpha\left(t^{k}\right)=\sum_{k \in \mathbb{N}} \frac{1}{k !} \cdot \tau^{k}(u) \cdot \alpha\left(t^{k}\right)=\left(\sum_{k \in \mathbb{N}} \frac{1}{k !} \cdot \alpha\left(t^{k}\right) \cdot \tau^{k}\right)(u)
$$

This means that $\alpha$ is decomposed into a series in terms of $\tau^{k}$ :

$$
\alpha=\sum_{k \in \mathbb{N}} \alpha_{k} \cdot \tau^{k}, \quad \alpha_{k}=\frac{1}{k !} \cdot \alpha\left(t^{k}\right)
$$

We can deduce from this that currents $\tau^{k}$ form a basis in the topological vector spaces $\mathcal{R}^{\star}(\mathbb{C})$ : every functional $\alpha \in \mathcal{R}^{\star}(\mathbb{C})$ can be uniquely represented by a converging in $\mathcal{R}^{\star}(\mathbb{C})$ series $($ (3.62), where coefficients $\alpha_{k} \in \mathbb{C}$ continuously depend on $\alpha \in \mathcal{R}^{\star}(\mathbb{C})$.

From (3.61) it follows that the action of the current $\alpha$ on a polynomial $u$ is defined by formula

$$
\alpha(u)=\sum_{k \in \mathbb{N}} \underbrace{\frac{1}{k !} \cdot \tau^{k}(u)}_{u_{k}} \cdot \underbrace{\frac{1}{k !} \cdot \alpha\left(t^{k}\right)}_{\alpha_{k}} \cdot k !=\sum_{k \in \mathbb{N}} u_{k} \cdot \alpha_{k} \cdot k !
$$

and the basis currents $\tau^{k}$ act on monomials $t^{k}$ by formula

$$
\tau^{k}\left(t^{n}\right)=\left\{\begin{array}{ll}
0, & n \neq k \\
n !, & n=k
\end{array},\right.
$$

This means that the system $\tau^{k}$ is not a dual basis for $t^{k}$ : it differs from the dual basis by the coefficients $k$ !. However, since $\mathcal{R}^{\star}(\mathbb{C}) \cong \mathbb{C}^{\mathbb{N}}$ (this follows from (3.54)) and, on the other hand, in $\mathbb{C}^{\mathbb{N}}$ any two bases are isomorphic (Theorem 0.20 ), we have

Proposition 3.11. The mapping

$$
\alpha \in \mathcal{R}^{\star}(\mathbb{C}) \mapsto\left\{\alpha_{k} ; k \in \mathbb{N}\right\} \in \mathbb{C}^{\mathbb{N}}
$$

is an isomorphism of topological vector spaces:

$$
\mathcal{R}^{\star}(\mathbb{C}) \cong \mathbb{C}^{\mathbb{N}}
$$

This isomorphism is not however, an isomorphism of algebras, since the multiplication in $\mathcal{R}^{\star}(\mathbb{C})$ is not coordinate-wise (i.e. not by formula (1.20), as it could defined in $\mathbb{C}^{\mathbb{N}}$ by analogy with the case of $\S 1$ (c) $)$, but as power series:

$$
\alpha * \beta=\sum_{k \in \mathbb{N}}\left(\sum_{i=0}^{k} \alpha_{k-i} \cdot \beta_{i}\right) \cdot \tau^{k},
$$

This follows from formula (3.66) below:

Proposition 3.12. The algebra $\mathcal{R}^{\star}(\mathbb{C})$ of currents of zero degree on the complex plane $\mathbb{C}$ is a nuclear Hopf-Fréchet algebra with the topology generated by seminorms

$$
\|\alpha\|_{K}=\sum_{k=0}^{K}\left|\alpha_{k}\right|, \quad(K \in \mathbb{N})
$$


and algebraic operations defined on basis elements $\tau^{k}$ by formulas

$$
\begin{array}{rlrl}
\tau^{k} * \tau^{l} & =\tau^{k+l} & 1_{\mathcal{R}^{\star}(\mathbb{C})}=\tau^{0} \\
\varkappa\left(\tau^{k}\right) & =\sum_{i=0}^{k}\left(\begin{array}{c}
k \\
i
\end{array}\right) \cdot \tau^{k-i} \circledast \tau^{i} & \varepsilon\left(\tau^{k}\right)=\left\{\begin{array}{l}
1, \\
0,
\end{array}\right. \\
\sigma\left(\tau^{k}\right)=(-1)^{k} \cdot \tau^{k} &
\end{array}
$$

Proof. There are many ways to prove these formulas, for instance, to prove (3.66) one can use formula (3.57) for comultiplication in $\mathcal{R}(\mathbb{C})$ :

$$
\begin{aligned}
& \left(\tau^{k} * \tau^{l}\right)\left(t^{m}\right)=\left(\tau^{k} \circledast \tau^{l}\right)\left(\varkappa\left(t^{m}\right)\right)=(\underline{3.57})=\left(\tau^{k} \circledast \tau^{l}\right)\left(\sum_{i=0}^{m}\left(\begin{array}{c}
m \\
i
\end{array}\right) \cdot t^{m-i} \odot t^{i}\right)=
\end{aligned}
$$

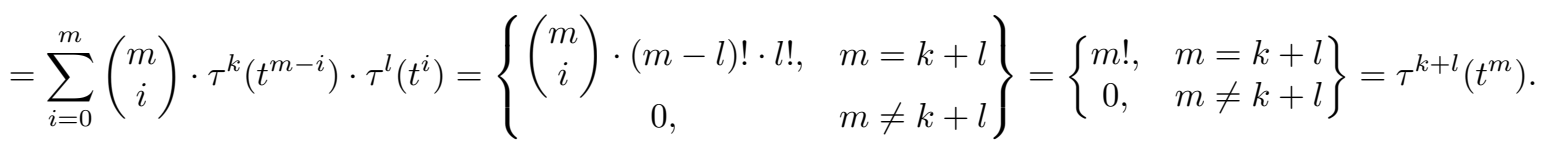

(on each monomial $t^{m}$ the action of functionals $\tau^{k} * \tau^{l}$ and $\tau^{k+l}$ coincide, hence they coincide themselves).

Let us consider now algebra $\mathcal{O}(\mathbb{C})$ of entire functions and its dial algebra $\mathcal{O}^{\star}(\mathbb{C})$ of analytical functionals on complex plane $\mathbb{C}$. Again, let $t^{k}$ denote the monomial of degree $k \in \mathbb{N}$ on $\mathbb{C}$, and $\tau^{k}$ the functional of taking $k$-th derivative in the point 0 :

$$
t^{k}(z)=z^{k} \quad \tau^{k}(u)=\left.\left(\frac{\mathrm{d}^{k}}{\mathrm{~d} z^{k}} u(z)\right)\right|_{z=0} \quad(x \in \mathbb{C}, \quad u \in \mathcal{O}(\mathbb{C}))
$$

Then it is convenient to represent elements $\mathcal{O}(\mathbb{C})$ and $\mathcal{O}^{\star}(\mathbb{C})$ as (converging in these spaces) series

$$
\begin{aligned}
& u \in \mathcal{O}(\mathbb{C}) \quad \Longleftrightarrow \quad u=\sum_{n=0}^{\infty} u_{n} \cdot t^{n}, \quad u_{n}=\frac{1}{n !} \tau^{n}(u) \in \mathbb{C}: \quad \forall C>0 \quad \sum_{n=0}^{\infty}\left|u_{n}\right| \cdot C^{n}<\infty \\
& \alpha \in \mathcal{O}^{\star}(\mathbb{C}) \quad \Longleftrightarrow \quad \alpha=\sum_{n=0}^{\infty} \alpha_{n} \cdot \tau^{n}, \quad \alpha_{n}=\frac{1}{n !} \alpha\left(t^{n}\right) \in \mathbb{C}: \quad \exists M, C>0 \quad \forall n \in \mathbb{N} \quad\left|\alpha_{n}\right| \leqslant M \cdot \frac{C^{n}}{n !}
\end{aligned}
$$

The action of an analytical functional $\alpha$ on an entire function $u$ is described by formula

$$
\langle u, \alpha\rangle=\sum_{n=0}^{\infty} u_{n} \cdot \alpha_{n} \cdot n !
$$

and the multiplications in $\mathcal{O}(\mathbb{C})$ and in $\mathcal{O}^{\star}(\mathbb{C})$ are defined by the same formulas as for the usual power series:

$$
u \cdot v=\sum_{n=0}^{\infty}\left(\sum_{i=0}^{n} u_{i} \cdot v_{n-i}\right) \cdot t^{n}, \quad \alpha * \beta=\sum_{k \in \mathbb{N}}\left(\sum_{i=0}^{k} \alpha_{i} \cdot \beta_{k-i}\right) \cdot \tau^{k},
$$

Proposition 3.13. The algebra $\mathcal{O}(\mathbb{C})$ of entire functions on complex plane $\mathbb{C}$ is a nuclear Hopf-Fréchet algebra with the topology generated by seminorms

$$
\|u\|_{C}=\sum_{k \in \mathbb{N}}\left|u_{k}\right| \cdot C^{k} \quad(C \geqslant 1)
$$


and algebraic operations defined on monomials $t^{k}$ by the same formulas $(\underline{3.56})-(\sqrt[3.58]{)}$ as for $\mathcal{R}(\mathbb{C})$ :

$$
\begin{array}{ll}
\mu\left(t^{k} \odot t^{l}\right)=t^{k+l} & 1_{\mathcal{R}(\mathbb{C})}=t^{0} \\
\varkappa\left(t^{k}\right)=\sum_{i=0}^{k}\left(\begin{array}{c}
k \\
i
\end{array}\right) \cdot t^{k-i} \odot t^{i} & \varepsilon\left(t^{k}\right)= \begin{cases}1, & k=0 \\
0, & k>0\end{cases} \\
\sigma\left(t^{k}\right)=(-1)^{k} \cdot t^{k} &
\end{array}
$$

Proof. The formulas for algebraic operations are proved similarly with the same formulas for $\mathcal{R}(\mathbb{C})$, so it remains only to explain, why the topology is generated by seminorms (3.72). This is a subject of mathematical folklore (see e.g. [26]): clearly, every usual seminorm $|u|_{K}=\max _{z \in K}|u(z)|$, where $K$ is a compact set in $\mathbb{C}$, is majorized by some seminorm $\|u\|_{C}$, namely by the one with $C=\max _{z \in K}|z|$ :

$$
|u|_{K}=\max _{z \in K}|u(z)|=\max _{z \in K}\left|\sum_{n=0}^{\infty} u_{n} \cdot z^{n}\right| \leqslant \max _{z \in K} \sum_{n=0}^{\infty}\left|u_{n}\right| \cdot\left|z^{n}\right| \leqslant \sum_{n=0}^{\infty}\left|u_{n}\right| \cdot C^{n}=\|u\|_{C}
$$

On the contrary, for every $C>0$ we can take a compact set $K=\{z \in \mathbb{C}:|z| \leqslant C+1\}$, and then from formulas for Cauchy coefficients

$$
\left|u_{n}\right| \leqslant \frac{|u|_{K}}{(C+1)^{n}}
$$

it follows that $\|u\|_{C}$ is subordinated to $|u|_{K}$ (with the constant $C+1$ ):

$$
\|u\|_{C}=\sum_{n=0}^{\infty}\left|u_{n}\right| \cdot C^{n} \leqslant \sum_{n=0}^{\infty} \frac{|u|_{K}}{(C+1)^{n}} \cdot C^{n} \leqslant|u|_{K} \cdot \sum_{n=0}^{\infty}\left(\frac{C}{C+1}\right)^{n}=|u|_{K} \cdot \frac{1}{1-\frac{C}{C+1}}=(C+1) \cdot|u|_{K}
$$

Proposition 3.14. The algebra $\mathcal{O}^{\star}(\mathbb{C})$ of analytical functionals on complex plane $\mathbb{C}$ is a nuclear HopfBrauner algebra with the topology generated by seminorms

$$
\left\|\left|\alpha \|_{r}=\sum_{k \in \mathbb{N}} r_{k} \cdot\right| \alpha_{k} \mid \cdot k ! \quad\left(r_{k} \geqslant 0: \forall C>0 \sum_{k \in \mathbb{N}} r_{k} \cdot C^{k}<\infty\right)\right.
$$

and algebraic operations defined on basis elements $\tau^{k}$ by the same formulas (3.66)-(3.68) as in the case of $\mathcal{R}^{\star}(\mathbb{C})$ :

$$
\begin{array}{ll}
\mu\left(\tau^{k} \circledast \tau^{l}\right)=\tau^{k+l} & 1_{\mathcal{R}^{\star}(\mathbb{C})}=\tau^{0} \\
\varkappa\left(\tau^{k}\right)=\sum_{i=0}^{k}\left(\begin{array}{c}
k \\
i
\end{array}\right) \cdot \tau^{k-i} \circledast \tau^{i} & \varepsilon\left(\tau^{k}\right)= \begin{cases}1, & k=0 \\
0, & k>0\end{cases} \\
\sigma\left(\tau^{k}\right)=(-1)^{k} \cdot \tau^{k} &
\end{array}
$$

Proof. Here again the formulas for algebraic operations are proved similarly with the case of $\mathcal{R}^{\star}(\mathbb{C})$, so we need only to explain, why the topology is generated by seminorms (3.73). Note first that for any sequence of non-negative numbers $r_{k} \geqslant 0$, satisfying the condition

$$
\forall C>0 \quad \sum_{k \in \mathbb{N}} r_{k} \cdot C^{k}<\infty
$$

the set

$$
E_{r}=\left\{u \in \mathcal{O}(\mathbb{C}): \forall k \in \mathbb{N} \quad\left|u_{k}\right| \leqslant r_{k}\right\}
$$


is compact in $\mathcal{O}(\mathbb{C})$, since it is closed and is contained in the rectangle $f$, where

$$
f(x)=\sum_{k \in \mathbb{N}} r_{k} \cdot|x|^{k}, \quad x \in \mathbb{C}
$$

Hence, $E_{r}$ generates a continuous seminorm $\alpha \mapsto \max _{u \in E_{r}}|\langle u, \alpha\rangle|$ on $\mathcal{O}^{\star}(\mathbb{C})$. But this is exactly seminorm (3.73):

$$
\max _{u \in E_{r}}|\langle u, \alpha\rangle|=\max _{|u| \leqslant r_{k}}\left|\sum_{k \in \mathbb{N}} u_{k} \cdot \alpha_{k} \cdot k !\right|=\sum_{k \in \mathbb{N}} r_{k} \cdot\left|\alpha_{k}\right| \cdot k !=\||\alpha|\|_{r}
$$

It remains to verify that the seminorms \|\|$\cdot\|\|_{r}$ indeed generate the topology of the space $\mathcal{O}^{\star}\left(\mathbb{C}^{\times}\right)$. This follows from:

Lemma 3.3. If $p$ is a continuous seminorm on $\mathcal{O}^{\star}(\mathbb{C})$, then the numbers $r_{k}=\frac{1}{k !} p\left(\tau^{k}\right)$ satisfy the condition (3.74), and $p$ is majorized by the seminorm $\|\mid \cdot\| \|_{r}$ :

$$
p(\alpha) \leqslant\|\| \alpha \|_{r}
$$

Proof. The set

$$
D=\left\{u \in \mathcal{O}(\mathbb{C}): \sup _{\alpha \in \mathcal{O}^{*}(\mathbb{C}): p(\alpha) \leqslant 1}|\alpha(u)| \leqslant 1\right\}
$$

is compact in $\mathcal{O}(\mathbb{C})$ and generates the seminorm $p$ :

$$
p(\alpha)=\sup _{u \in D}|\alpha(u)|
$$

Hence

$$
r_{k}=\frac{1}{k !} p\left(\tau^{k}\right)=\frac{1}{k !} \sup _{u \in D}\left|\tau^{k}(u)\right|=\sup _{u \in D}\left|u_{k}\right|
$$

For each $C>0$ we have:

$$
\begin{gathered}
\infty>\sup _{u \in D}\|u\| \|_{C+1}=\sup _{u \in D} \sum_{k \in \mathbb{N}}\left|u_{k}\right| \cdot(C+1)^{k} \geqslant \sup _{u \in D} \sup _{k}\left|u_{k}\right| \cdot(C+1)^{k}=\sup _{k}\left(r_{k} \cdot(C+1)^{k}\right) \\
\Downarrow \\
\exists M>0 \quad \forall k \in \mathbb{N} \quad r_{k} \leqslant \frac{M}{(C+1)^{k}} \\
\Downarrow \quad \sum_{k \in \mathbb{N}} r_{k} \cdot C^{k} \leqslant \sum_{k \in \mathbb{N}} \frac{M}{(C+1)^{k}} \cdot C^{k}<\infty
\end{gathered}
$$

I.e., $r_{k}$ indeed satisfy condition (3.74). Formula (3.76) is proved by the chain of inequalities analogous to (3.29).

Proposition 3.15. The mappings

$$
t^{k} \mapsto t^{k} \mapsto \tau^{k} \mapsto \tau^{k} \quad(k \in \mathbb{N})
$$

define the chain of homomorphisms of rigid Hopf algebras:

$$
\mathcal{R}(\mathbb{C}) \rightarrow \mathcal{O}(\mathbb{C}) \rightarrow \mathcal{O}^{\star}(\mathbb{C}) \rightarrow \mathcal{R}^{\star}(\mathbb{C})
$$




\section{$\S 4$ Functions of exponential type on a Stein group}

\section{(a) Semicharacters and inverse semicharacters on Stein groups}

Let $G$ be a Stein group. Then

- a locally bounded function $f: G \rightarrow[1,+\infty)$ is called a semicharacter, if it satisfies the following so called submultiplicativity inequality:

$$
f(x \cdot y) \leqslant f(x) \cdot f(y), \quad x, y \in G
$$

- a function $\varphi: G \rightarrow(0 ; 1]$, locally separated from zero, is called inverse semicharacter, if it satisfies the inverse inequality:

$$
\varphi(x) \cdot \varphi(y) \leqslant \varphi(x \cdot y), \quad x, y \in G
$$

Clearly, if $f: G \rightarrow[1,+\infty)$ is a semicharacter, then the inverse function

$$
\varphi(x)=\frac{1}{f(x)}
$$

is an inverse semicharacter, and vice versa.

\section{Properties of semicharacters and inverse semicharacters:}

(i) The set of all semicharacters on $G$ is closed under the following operations:

- multiplication by a sufficiently big constant: $C \cdot f \quad(C \geqslant 1)$,

- multiplication: $f \cdot g$,

— addition: $f+g$,

— taking maximum: $\max \{f, g\}$.

(ii) The set of all inverse semicharacters on $G$ is closed under the following operations:

- multiplication by a sufficiently small constant: $C \cdot \varphi \quad(C \leqslant 1)$,

- multiplication: $\varphi \cdot \psi$,

— taking half of harmonic mean: $\frac{\varphi \cdot \psi}{\varphi+\psi}$

— taking minimum: $\min \{\varphi, \psi\}$.

Proof. Having in mind the duality between semicharacters and inverse semicharacters reflected by formula (4.3), we can consider only the case of semicharacters.

If $f$ is a semicharacter on $G$ and $C \geqslant 1$, then

$$
C \cdot f(x \cdot y) \leqslant C \cdot f(x) \cdot f(y) \leqslant(C \cdot f(x)) \cdot(C \cdot f(y))
$$

If $f$ and $g$ are semicharacters on $G$, then considering their multiplication we have:

$$
\begin{aligned}
(f \cdot g)(x \cdot y)=f(x \cdot y) \cdot g(x \cdot y) \leqslant f(x) \cdot f(y) \cdot g(x) & \cdot g(y)= \\
& =f(x) \cdot g(x) \cdot f(y) \cdot g(y)=(f \cdot g)(x) \cdot(f \cdot g)(y)
\end{aligned}
$$

For their sum we have:

$$
\begin{aligned}
& (f+g)(x \cdot y)=f(x \cdot y)+g(x \cdot y) \leqslant f(x) \cdot f(y)+g(x) \cdot g(y) \leqslant \\
\leqslant & f(x) \cdot f(y)+g(x) \cdot f(y)+f(x) \cdot g(y)+g(x) \cdot g(y)=(f(x)+g(x)) \cdot(f(y)+g(y))=(f+g)(x) \cdot(f+g)(y)
\end{aligned}
$$


The proof for maximum $\max \{f, g\}$ is based on the following evident inequality:

$$
\max \{a \cdot b, c \cdot d\} \leqslant \max \{a, c\} \cdot \max \{b, d\} \quad(a, b, c, d>0)
$$

It implies:

$$
\begin{aligned}
\max \{f, g\}(x \cdot y)=\max \{f(x \cdot y) & , g(x \cdot y)\} \leqslant \max \{f(x) \cdot f(y), g(x) \cdot g(y)\} \leqslant(4.4) \leqslant \\
& \leqslant \max \{f(x), g(x)\} \cdot \max \{f(y), g(y)\}=\max \{f, g\}(x) \cdot \max \{f, g\}(y)
\end{aligned}
$$

Example 4.1. All the submultiplicative matrix norms (see [15]), for instance

$$
\|x\|=\sum_{i, j=1}^{n}\left|x_{i, j}\right|, \quad\|x\|=\sqrt{\sum_{i, j=1}^{n}\left|x_{i, j}\right|^{2}}
$$

are semicharacters on $\mathrm{GL}_{n}(\mathbb{C})$. From the properties of semicharacters it follows that the function of the form

$$
r_{C}^{N}(x)=C \cdot \max \left\{\|x\| ;\left\|x^{-1}\right\|\right\}^{N}, \quad C \geqslant 1, N \in \mathbb{N}
$$

are again semicharacters on $\mathrm{GL}_{n}(\mathbb{C})$.

Proposition 4.1. For any submultiplicative matrix norm $\|\cdot\|$ on $\mathrm{GL}_{n}(\mathbb{C})$ the semicharacters of the form (4.6) majorize all other semicharacters on $\mathrm{GL}_{n}(\mathbb{C})$.

Proof. Note from the very beginning that it is sufficient to consider the case when $\|\cdot\|$ is the Euclid norm on the algebra $\mathrm{M}_{n}(\mathbb{C})$ of all complex matrices $n \times n$ :

$$
\|x\|=\sup _{\xi \in \mathbb{C}^{n}: \| \xi \mid \leqslant 1}\|x(\xi)\|, \quad \text { where } \quad\|\xi\|=\sqrt{\sum_{i=1}^{n}\left|\xi_{i}\right|^{2}}
$$

- since any other norm on $\mathrm{M}_{n}(\mathbb{C})$ majorizes the Euclid seminorm up to a constant multiplier, this will prove our proposition.

Consider the set

$$
K:=\left\{x \in \mathrm{GL}_{n}(\mathbb{C}): \max \left\{\|x\| ;\left\|x^{-1}\right\|\right\} \leqslant 2\right\}=\left\{x \in \mathrm{GL}_{n}(\mathbb{C}): \forall \xi \in \mathbb{C}^{n} \quad \frac{1}{2} \cdot\|\xi\| \leqslant\|x(\xi)\| \leqslant 2 \cdot\|\xi\|\right\}
$$

It is closed and bounded in the algebra of matrices $\mathrm{M}_{n}(\mathbb{C})$, hence it is compact. This is a generating compact in $\mathrm{GL}_{n}(\mathbb{C})$

$$
\mathrm{GL}_{n}(\mathbb{C})=\bigcup_{m=1}^{\infty} K^{m}, \quad K^{m}=\underbrace{K \cdot \ldots \cdot K}_{m \text { factors }},
$$

In addition,

$$
K^{m}=\left\{x \in \mathrm{GL}_{n}(\mathbb{C}): \max \left\{\|x\| ;\left\|x^{-1}\right\|\right\}^{m} \leqslant 2^{m}\right\}
$$

Indeed, if $x \in K^{m}$, then $x=x_{1} \cdot \ldots \cdot x_{m}$, where $\left\|x_{i}\right\| \leqslant 2$ and $\left\|x_{i}^{-1}\right\| \leqslant 2$, hence

$$
\|x\| \leqslant \prod_{i=1}^{m}\left\|x_{i}\right\| \leqslant 2^{m}, \quad\left\|x^{-1}\right\| \leqslant \prod_{i=1}^{m}\left\|x_{i}^{-1}\right\| \leqslant 2^{m}
$$

On the contrary, suppose $\max \left\{\|x\|^{m} ;\left\|x^{-1}\right\|^{m}\right\} \leqslant 2^{m}$. Consider the polar decomposition: $x=r \cdot u$, where $r$ is a positive definite Hermitian, and $u$ a unitary matrices. Let us decompose $r$ into a product $r=v \cdot d \cdot v^{-1}$, where $v$ is unitary, and $d$ a diagonal matrices:

$$
d=\left(\begin{array}{cccc}
d_{1} & 0 & \ldots & 0 \\
0 & d_{2} & \ldots & 0 \\
\ldots & \ldots & \ldots & \ldots \\
0 & 0 & \ldots & d_{n}
\end{array}\right), \quad d_{i} \geqslant 0
$$


The $m$-th root

$$
\sqrt[m]{d}=\left(\begin{array}{cccc}
\sqrt[m]{d_{1}} & 0 & \ldots & 0 \\
0 & \sqrt[m]{d_{2}} & \ldots & 0 \\
\ldots & \ldots & \ldots & \ldots \\
0 & 0 & \ldots & \sqrt[m]{d_{n}}
\end{array}\right)
$$

has the following properties:

$$
\begin{gathered}
\max _{i=1, \ldots, n} d_{i}=\|d\|=\|r\|=\|x\| \leqslant 2^{m} \quad \Longrightarrow \quad\|\sqrt[m]{d}\|=\max _{i=1, \ldots, n} \sqrt[m]{d_{i}} \leqslant 2 \\
\max _{i=1, \ldots, n} d_{i}^{-1}=\left\|d^{-1}\right\|=\left\|r^{-1}\right\|=\left\|x^{-1}\right\| \leqslant 2^{m} \quad \Longrightarrow \quad\left\|\sqrt[m]{d^{-1}}\right\|=\max _{i=1, \ldots, n} \sqrt[m]{d_{i}^{-1}} \leqslant 2
\end{gathered}
$$

As a corollary, the matrix

$$
y=v \cdot \sqrt[m]{d} \cdot v^{-1}
$$

belongs to $K$ :

$$
\max \left\{\|y\| ;\left\|y^{-1}\right\|\right\}=\max \left\{\|\sqrt[m]{d}\| ;\left\|\sqrt[m]{d}^{-1}\right\|\right\} \leqslant 2 \quad \Longrightarrow \quad y \in K
$$

Hence the matrix $y \cdot u$ also belongs to $K$, and we obtain

$$
x=v \cdot d \cdot v^{-1} \cdot u=v \cdot(\sqrt[m]{d})^{m-1} \cdot v^{-1} \cdot v \cdot(\sqrt[m]{d}) \cdot v^{-1} \cdot u=\underbrace{y^{m-1}}_{\substack{\Re \\ K^{m-1}}} \cdot \underbrace{y \cdot v}_{K} \in K^{m}
$$

We have proved formula (4.8). Now let $f$ be an arbitrary semicharacter on $\mathrm{GL}_{n}(\mathbb{C})$. Put

$$
C=\sup _{x \in K} f(x), \quad N \geqslant \log _{2} C
$$

and let us show that

$$
\forall x \in \mathrm{GL}_{n}(\mathbb{C}) \quad f(x) \leqslant r_{C}^{N}(x)
$$

Take $x \in \mathrm{GL}_{n}(\mathbb{C})$. From (4.7) it follows that $x \in K^{m} \backslash K^{m-1}$ for some $m \in \mathbb{N}$. By formula (4.8) we have:

$$
\begin{gathered}
2^{m-1}<\max \left\{\|x\| ;\left\|x^{-1}\right\|\right\} \leqslant 2^{m} \\
\Downarrow \\
m-1<\log _{2}\left(\max \left\{\|x\| ; \| x^{-1}||\right\}\right) \\
\Downarrow \\
f(x) \leqslant \sup _{y \in K} f(y)^{m} \leqslant C^{m} \leqslant C \cdot C^{m-1}<C \cdot C^{\log _{2}\left(\max \left\{\|x\| ;\left\|x^{-1}\right\|\right\}\right)}= \\
=C \cdot\left(\max \left\{\|x\| ;\left\|x^{-1}\right\|\right\}\right)^{\log _{2} C} \leqslant C \cdot\left(\max \left\{\|x\| ;\left\|x^{-1}\right\|\right\}\right)^{N}=r_{C}^{N}(x)
\end{gathered}
$$

In the special case when $n=1$ we have:

Corollary 4.1. On the complex circle $\mathbb{C}^{\times}$the semicharacters of the form

$$
r_{C}^{N}(t)=C \cdot \max \left\{|t| ;|t|^{-1}\right\}^{N}, \quad C \geqslant 1, N \in \mathbb{N}
$$

majorize all other semicharacters. 
Proposition 4.2. If $G$ is a compactly generated Stein group and $K$ a compact neighborhood of identity in $G$, generating $G$,

$$
G=\bigcup_{n=1}^{\infty} K^{n}, \quad K^{n}=\underbrace{K \cdot \ldots \cdot K}_{n \text { factors }}
$$

then for any $C \geqslant 1$ the rule

$$
h_{C}(x)=C^{n} \Longleftrightarrow x \in K^{n} \backslash K^{n-1}
$$

defines a semicharacter $h_{C}$ on $G$. Such semicharacters form a fundamental system among all semicharacters on $G$ : every semicharacter $f$ on $G$ is majorized by some semicharacter $h_{C}$

$$
f(x) \leqslant h_{C}(x), \quad x \in G
$$

- for this the constant $C$ should be chosen such that

$$
C \geqslant \max _{t \in K} f(t)
$$

Proof. The local boundedness of $h_{C}$ is obvious, so we need to check the submultiplicativity inequality. Take $x, y \in G$ and choose $k, l \in \mathbb{N}$ such that

$$
x \in K^{k} \backslash K^{k-1}, \quad y \in K^{l} \backslash K^{l-1} .
$$

Then $x \cdot y \in K^{k+l}$, so

$$
h_{C}(x \cdot y) \leqslant C^{k+l}=C^{k} \cdot C^{l}=h_{C}(x) \cdot h_{C}(y)
$$

If now $f$ is an arbitrary semicharacter, and $C$ satisfies the condition (4.11), then

$$
x \in K^{n} \backslash K^{n-1} \Longrightarrow f(x) \leqslant\left(\max _{t \in K} f(t)\right)^{n}=C^{n}=h_{C}(x)
$$

\section{(b) Submultiplicative rhombuses and dually submultiplicative rectangles}

Let us introduce the following definitions:

- a closed absolutely convex neighborhood of zero $\Delta$ in $\mathcal{O}^{\star}(G)$ is said to be submultiplicative, if for any functionals $\alpha, \beta$ from $\Delta$ their convolution $\alpha * \beta$ also belong to $\Delta$ :

$$
\forall \alpha, \beta \in \Delta \quad \alpha * \beta \in \Delta
$$

shortly this is expressed by the following inclusion:

$$
\Delta * \Delta \subseteq \Delta
$$

- an absolutely closed compact set $D$ in $\mathcal{O}(G)$ is said to be dually submultiplicative, if its polar $D^{\circ}$ is a submultiplicative neighborhood of zero:

$$
D^{\circ} * D^{\circ} \subseteq D^{\circ}
$$

\section{Lemma 4.1.}

(a) If $\varphi: G \rightarrow(0 ; 1]$ is an inverse semicharacter on $G$, then its rhombus $\varphi^{\star}$ is a (closed, absolutely convex, and) submultiplicative neighborhood of zero in $\mathcal{O}^{\star}(G)$.

(b) If $\Delta \subseteq \mathcal{O}^{\star}(G)$ is a closed absolutely convex and submultiplicative neighborhood of zero in $\mathcal{O}^{\star}(G)$, then its inner envelope $\Delta^{\diamond}$ is an inverse semicharacter on $G$. 
Proof. 1. Let us denote

$$
\varepsilon_{x}=\varphi(x) \cdot \delta^{x} \in \mathcal{O}^{\star}(G),
$$

then

$$
\varphi^{\Downarrow}=\overline{\operatorname{absconv}}\left\{\varphi(x) \cdot \delta^{x} ; \quad x \in M\right\}=\overline{\operatorname{absconv}}\left\{\varepsilon_{x} ; \quad x \in M\right\}
$$

and the inclusion

$$
\varphi^{\bullet} * \varphi^{\downarrow} \subseteq \varphi^{\downarrow}
$$

is verified in three steps. First we should note that

$$
\forall x, y \in G \quad \varepsilon_{x} * \varepsilon_{y} \in \varphi^{\bullet}
$$

Indeed,

$$
\varepsilon_{x} * \varepsilon_{y}=\varphi(x) \cdot \delta^{x} * \varphi(y) \cdot \delta^{y}=\varphi(x) \cdot \varphi(y) \cdot \delta^{x \cdot y}=\underbrace{\frac{\varphi(x) \cdot \varphi(y)}{\varphi(x \cdot y)}}_{\substack{\wedge \\ 1}} \cdot \varepsilon_{x \cdot y} \in \overline{\operatorname{absconv}}\left\{\varepsilon_{z} ; \quad z \in M\right\}=\varphi
$$

Second, we take finite absolutely convex combinations of functionals $\varepsilon_{x}$ :

$$
\alpha=\sum_{i=1}^{k} \lambda_{i} \cdot \varepsilon_{x_{i}}, \quad \beta=\sum_{j=1}^{l} \mu_{j} \cdot \varepsilon_{y_{j}} \quad\left(\sum_{i=1}^{k}\left|\lambda_{i}\right| \leqslant 1, \quad \sum_{j=1}^{l}\left|\mu_{j}\right| \leqslant 1\right)
$$

For them we have:

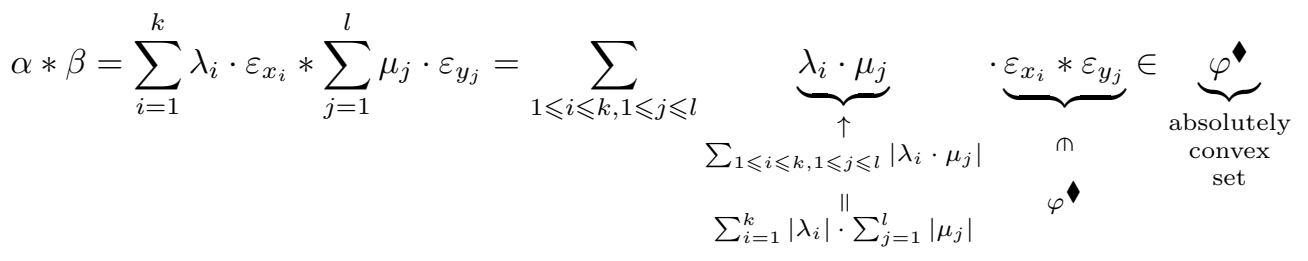

$$
\begin{aligned}
& \stackrel{1}{1}
\end{aligned}
$$

And, third, for arbitrary $\alpha, \beta \in \varphi$ the inclusion $\alpha * \beta \in \varphi$ becomes a corollary of two facts: that functionals (4.13) are dense in the set $\varphi^{\star}=\overline{\operatorname{absconv}}\left\{\varepsilon_{x} ; x \in M\right\}$, and that the convolution $*$ is continuous.

2. We use here formula (2.19):

$$
\begin{aligned}
& \Delta * \Delta \subseteq \Delta \quad \Longrightarrow \quad \forall x, y \in G \quad \underbrace{\Delta^{\diamond}(x) \cdot \delta^{x}} * \underbrace{\Delta^{\diamond}(y) \cdot \delta^{y}}=\Delta^{\diamond}(x) \cdot \Delta^{\diamond}(y) \cdot \delta^{x \cdot y} \in \Delta \quad \Longrightarrow
\end{aligned}
$$

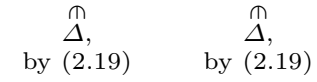

$$
\begin{aligned}
& \Longrightarrow \quad \Delta^{\diamond}(x) \cdot \Delta^{\diamond}(y) \leqslant \max \left\{\lambda>0: \lambda \cdot \delta^{x \cdot y} \in \Delta\right\}=\underline{(2.19)}=\Delta^{\diamond}(x \cdot y) \quad \Longrightarrow \quad \Delta^{\diamond}(x) \cdot \Delta^{\diamond}(y) \leqslant \Delta^{\diamond}(x \cdot y)
\end{aligned}
$$

\section{Lemma 4.2 .}

(a) If $f: G \rightarrow[1 ; \infty)$ is a semicharacter on $G$, then its rectangle $f \boldsymbol{\bullet}$ is dually submultiplicative.

(b) If $D \subseteq \mathcal{O}(G)$ is a dually submultiplicative absolutely convex compacts set, then its outer envelope $D^{\square}$ is a semicharacter on $G$. 
Proof. 1. If $f: G \rightarrow[1 ; \infty)]$ is a semicharacter, then $\frac{1}{f}$ is an inverse semicharacter, therefore by Lemma 4.1 (a) the rhombus

$$
\left(\frac{1}{f}\right)^{\bullet}=(2.28)=\left(f^{\natural}\right)^{\circ}
$$

is a submultiplicative neighborhood of zero. This means that $f \mathbf{\square}$ is dually submultiplicative.

2. If $D \subseteq \mathcal{O}(G)$ is a dually submultiplicative absolutely convex compact set, then its polar $D^{\circ} \subseteq \mathcal{O}^{\star}(G)$ is a submultiplicative neighborhood of zero in $\mathcal{O}^{\star}(G)$, hence by Lemma 4.1 (b) the inner envelope

$$
\left(D^{\circ}\right)^{\diamond}=(\underline{2.29})=\frac{1}{D^{\square}}
$$

is an inverse semicharacter. Therefore, $D^{\square}$ must be a semicharacter.

Lemmas 4.1 and 4.2 together with formulas $\Delta=\Delta^{\diamond}$ and $D=D^{\square}$ for rhombuses and rectangles give the following

\section{Theorem 4.1.}

(a) A rhombus $\Delta$ in $\mathcal{O}^{\star}(G)$ is submultiplicative if and only if its inner envelope $\Delta^{\diamond}$ is an inverse semicharacter on $G$.

(b) A rectangle $D$ in $\mathcal{O}(G)$ is dually submultiplicative if and only if its outer envelope $D^{\square}$ is a semicharacter on $G$.

The following result shows that the submultiplicative rhombuses form a fundamental system among all submultiplicative closed absolutely convex neighborhood of zero in $\mathcal{O}^{\star}(G)$ :

\section{Theorem 4.2.}

(a) Every closed absolutely convex neighborhood of zero $\Delta$ in $\mathcal{O}^{\star}(G)$ contains some submultiplicative rhombus, namely $\Delta^{\diamond}$.

(b) Every dually submultiplicative absolutely convex compact set $D$ in $\mathcal{O}(G)$ is contained in some dually submultiplicative rectangle, namely in $D^{\square}$.

Proof. 1. If $\Delta$ is a closed absolutely convex neighborhood of zero in $\mathcal{O}^{\star}(G)$, then by Lemma 4.1 (b), $\Delta^{\diamond}$ is an inverse semicharacter, hence by Lemma 4.1(a), the rhombus $\Delta^{\diamond}$ is submultiplicative.

2. If $D$ is a dually submultiplicative absolutely convex compact set in $\mathcal{O}(G)$, then its polar $D^{\circ}$ is a closed absolutely convex submultiplicative neighborhood of zero in $\mathcal{O}^{\star}(G)$, and by what we have already proved $\left(D^{\circ}\right)^{\diamond}$ is a submultiplicative rhombus. I.e. the set $\left(D^{\square}\right)^{\circ}=\left(D^{\circ}\right)^{\diamond}$ is submultiplicative. Therefore the rectangle $D^{\square}$ is dually submultiplicative.

In accordance with the definitions of $\S 2$, we call a function $f$ on $G$ an enveloping semicharacter, if it is an outer envelope and at the same time a semicharacter on $G$.

Theorem 4.3. If $G$ is a compactly generated Stein group, then the systems of all semicharacters, all enveloping semicharacters and all dual submultiplicative rectangles in $G$ contain countable cofinal subsystems:

- there exists a sequence $h_{N}$ of semicharacters on $G$ such that every semicharacter $g$ is majorized by some semicharacter $h_{N}$ :

$$
g(x) \leqslant h_{N}(x), \quad x \in G
$$

- there exists a sequence $f_{N}$ of enveloping semicharacters on $G$ such that every enveloping semicharacter $g$ is majorized by some semicharacter $f_{N}$ :

$$
g(x) \leqslant f_{N}(x), \quad x \in G
$$


- there exists a sequence $E_{N}$ of dually submupltiplicative rectangles in $G$ such that every dually submultiplicative rectangle $D$ in $G$ is contained in some $E_{N}$ :

$$
D \subseteq E_{N}
$$

Proof. This follows from Proposition 4.2 the semicharacters $h_{N}, N \in \mathbb{N}$, defined in (4.10), are the sequence we look for. The sequences $E_{N}$ and $f_{N}$ are defined as follows:

$$
\begin{gathered}
E_{N}=\left\{u \in \mathcal{O}(G): \max _{x \in K^{n}}|u(x)| \leqslant N^{n}\right\}=\left(h_{N}\right)^{】} \\
f_{N}=\left(E_{N}\right)^{\square}=\left(h_{N}\right)^{\mathbf{\square}}
\end{gathered}
$$

$\left(E_{N}\right.$ are dually submultiplicative rectangles by Lemma 4.2 (a), and $f_{N}$ are enveloping semicharacters by Lemma $4.2(\mathrm{~b}))$.

If now $D$ is a dually submultiplicative rectangle, then by Lemma $4.2(\mathrm{~b})$, its outer envelope $D^{\square}$ is a semicharacter, hence by Proposition 4.2, there is $N \in \mathbb{N}$ such that

$$
D^{\square} \leqslant h_{N}
$$

This means that $D$ is contained in some $E_{N}$ :

$$
D=\left(D^{\square}\right) \subseteq\left(h_{N}\right)^{\mathbf{\bullet}}=E_{N}
$$

This in its turn implies that

$$
D^{\square} \leqslant\left(E_{N}\right)^{\square}=f_{N},
$$

- so every enveloping semicharacter (always being of the form $D^{\square}$ by Theorem 4.1) is majorized by some $f_{N}$.

\section{(c) Holomorphic functions of exponential type}

Algebra $\mathcal{O}_{\exp }(G)$ of holomorphic functions of exponential type. A holomorphic function $u \in$ $\mathcal{O}(G)$ on a compactly generated Stein group $G$ we call a function of exponential type, if it is bounded by some semicharacter:

$$
|u(x)| \leqslant f(x), \quad x \in G \quad(f(x \cdot y) \leqslant f(x) \cdot f(y))
$$

The set of all holomorphic functions of exponential type on $G$ is denoted by $\mathcal{O}_{\exp }(G)$. It is a subspace in $\mathcal{O}(G)$ and, by Theorem 4.2. $\mathcal{O}_{\exp }(G)$ can be considered as the union of all dually submultiplicative rectangles in $\mathcal{O}(G)$ :

$$
\mathcal{O}_{\exp }(G)=\bigcup_{\begin{array}{c}
D \text { is a dually } \\
\text { submultiplicative } \\
\text { rectangle in } \mathcal{O}(G)
\end{array}} D=\bigcup_{f \text { is a semicharacter in } G} f
$$

or, what is the same, the union of all subspaces of the form $\mathbb{C} D$, where $D$ is a dually submultiplicative rectangle in $\mathcal{O}(G)$ :

$$
\mathcal{O}_{\exp }(G)=\bigcup_{\begin{array}{c}
D \text { is a dually } \\
\text { submultiplicative } \\
\text { rectangle in } \mathcal{O}(G)
\end{array}} \mathbb{C} D
$$

This equality allows to endow $\mathcal{O}_{\text {exp }}(G)$ with the natural topology - the topology of injective (locally convex) limit of Smith spaces $\mathbb{C} D$ :

$$
\mathcal{O}_{\exp }(G)=\lim _{\substack{D \text { is a dually } \\ \text { submultiplicative } \\ \text { rectangle in } \mathcal{O}(G)}} \mathbb{C} D
$$


From Theorem 4.3 it follows that in this limit the system of all dually submultiplicative rectangles can be repalced by some countable subsystem:

$$
\mathcal{O}_{\exp }(G)=\underset{N \rightarrow \infty}{\lim } \mathbb{C} E_{N}
$$

Together with Theorem 0.6 this gives the following fact.

Theorem 4.4. The space $\mathcal{O}_{\exp }(G)$ of the functions of exponential growth on a compactly generated Stein group $G$ is a Brauner space.

Corollary 4.2. If $G$ is a compactly generated Stein group, then every bounded set $D$ in $\mathcal{O}_{\exp }(G)$ is contained in some rectangle of the form $f^{\mathbf{\downarrow}}$, where $f$ is some semicharacter on $G$ :

$$
D \subseteq f
$$

Proof. By Proposition 0.1, $D$ is contained in one of the compact sets $E_{N}$. This set, being a dually submultiplicative rectangle, by Theorem 4.1 has the form $f_{N}^{\mathbf{\Lambda}}$ for some semicharacter $f_{N}$, namely for $f_{N}=E_{N}^{\square}$.

Theorem 4.5. The space $\mathcal{O}_{\exp }(G)$ of the functions of exponential growth on a compactly generated Stein group $G$ is a projective stereotype algebra with respect to usual pointwise multiplication of functions.

Proof. Note that if two functions $u, v \in \mathcal{O}(G)$ are bounded by semicharacters $f$ and $g$, then their multiplication $u \cdot v$ is bounded by the semicharacter $f \cdot g$ :

$$
u \in f \downarrow v \in g^{\mathbf{\Perp}} \Longrightarrow u \cdot v \in(f \cdot g)^{\mathbf{}}
$$

In other words, the multiplication $(u, v) \mapsto u \cdot v$ in the space $\mathcal{O}(G)$ turns any compact of the form $f \mathbf{\square} \times g \mathbf{\square}$ (where $f$ and $g$ are semicharacters) into the rectangle $(f \cdot g)$.

$$
(u, v) \in f^{\mathbf{\bullet}} \times g g^{\mathbf{\square}} \mapsto u \cdot v \in(f \cdot g) \mathbf{\square}
$$

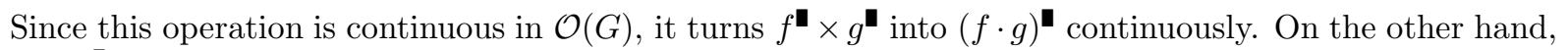
$(f \cdot g)$, being a dually submultiplicative rectangle, is continuously included into the space $\mathcal{O}_{\exp }(G)$. Thus we obtain a continuous mapping

$$
(u, v) \in f^{\mathbf{\bullet} \times g} \quad \mapsto \quad u \cdot v \in \mathcal{O}_{\exp }(G)
$$

If now $D$ and $E$ are arbitrary compact sets in the Brauner space $\mathcal{O}_{\text {exp }}(G)$, then by Corollary 4.2 they are contained in some compact sets of the form $f \mathbf{\bullet}$ and $g \boldsymbol{\Downarrow}$, where $f$ and $g$ are semicharacters. Hence,

$$
D \times E \subseteq f^{\mathbf{\square}} \times f^{\boldsymbol{\bullet}}
$$

From this we can conclude that the operation of multiplication is continuous from $D \times E$ into $\mathcal{O}_{\exp }(G)$ :

$$
(u, v) \in D \times E \subseteq f^{\mathbf{\bullet}} \times g \quad \mapsto u \cdot v \in \mathcal{O}_{\exp }(G)
$$

This is true for arbitrary compact sets $D$ and $E$ in $\mathcal{O}_{\exp }(G)$. So we can apply [1, Theorem 5.24]: since $\mathcal{O}_{\exp }(G)$, being a Brauner space, is co-complete (i.e. its stereotype dual space is complete), by 11, Theorem $2.4]$ it is saturated. This implies that any bilinear form on $\mathcal{O}_{\exp }(G)$, if continuous on compact sets of the form $D \times E$, will be continuous $\mathcal{O}_{\exp }(G)$ in the sense of definition [1, $\left.\S 5.6\right]$. Being applied to the operation $(u, v) \mapsto u \cdot v$ this means that the multiplication is continuous in the sense of conditions of Proposition 1.1. Hence, $\mathcal{O}_{\exp }(G)$ is a projective stereotype algebra.

Let us note the following obvious fact:

Theorem 4.6. If $H$ is a closed subgroup in a Stein group $G$, then the restriction of any holomorphic function of exponential type on $G$ to $H$ is a holomorphic function of exponential type:

$$
\left.u \in \mathcal{O}_{\exp }(G) \quad \Longrightarrow \quad u\right|_{H} \in \mathcal{O}_{\exp }(H)
$$


Algebra $\mathcal{O}_{\text {exp }}^{\star}(G)$ of exponential analytic functionals. We shall call the elements of the dual stereotype space $\mathcal{O}_{\text {exp }}^{\star}(G)$, i.e. linear continuous functionals on $\mathcal{O}_{\exp }(G)$ exponential analytic functionals on the group $G$. The space $\mathcal{O}_{\text {exp }}^{\star}(G)$ is called the space of exponential analytic functionals on $G$.

From Theorem 4.4 we have

Theorem 4.7. The space $\mathcal{O}_{\exp }(G)$ of exponential functionals on a compactly generated Stein group $G$ is a Fréchet space.

Theorem 4.8. The space $\mathcal{O}_{\text {exp }}^{\star}(G)$ of exponential functionals on a compactly generated Stein group $G$ is a projective stereotype algebra with respect to usual convolution of functionals defined by Formulas (3.10) $-(3.14)$ :

$$
(\alpha, \beta) \quad \mapsto \alpha * \beta
$$

Proof. 1. Take a function $u \in \mathcal{O}_{\exp }(G)$ and note that for any point $s \in G$ its shift $u \cdot s$ (defined by (3.10) ) is again a function from $\mathcal{O}_{\exp }(G)$ :

$$
\forall s \in G \quad \forall u \in \mathcal{O}_{\exp }(G) \quad u \cdot s \in \mathcal{O}_{\exp }(G)
$$

Indeed, if we take a semicharacter $f$ majorizing $u$, we obtain:

$$
\forall t \in G \quad|(u \cdot s)(t)|=|u(s \cdot t)| \leqslant f(s \cdot t) \leqslant f(s) \cdot f(t)
$$

i.e.,

$$
u \in f \quad \Longrightarrow \quad u \cdot s \in f(s) \cdot f \mathbf{\downarrow}
$$

2. Let us denote the mapping $s \mapsto u \cdot s$ by $\widehat{u}$,

$$
\widehat{u}: G \rightarrow \mathcal{O}_{\exp }(G) \quad \mid \widehat{u}(s):=u \cdot s, \quad s \in G
$$

and show that it is continuous. Let $s_{i}$ be a sequence of points in $G$, tending to a point $s$ :

$$
s_{i} \underset{i \rightarrow \infty}{\stackrel{G}{\longrightarrow}} s
$$

Then the sequence of holomorphic functions $\widehat{u}\left(s_{i}\right) \in \mathcal{O}(G)$ tends to the holomorphic function $\widehat{u}(s) \in \mathcal{O}(G)$ uniformly on every compact set $K \subseteq G$, i.e. in the space $\mathcal{O}(G)$ :

$$
\widehat{u}\left(s_{i}\right) \underset{i \rightarrow \infty}{\stackrel{\mathcal{O}(G)}{\longrightarrow}} \widehat{u}(s)
$$

At the same time, from (4.20) it follows that all those functions are bounded by the semicharacter $C \cdot f$, where $C=\max \left\{\sup _{i} f\left(s_{i}\right), f(s)\right\}$ (this value is finite since the sequence $s_{i}$ with its limit $s$ forms a compact set), so it lies in the rectangle generated by the semicharacter $C \cdot f$ :

$$
\widehat{u}\left(s_{i}\right)=u \cdot s_{i} \in C \cdot f \mathbf{\downarrow}, \quad \widehat{u}(s)=u \cdot s \in C \cdot f \boldsymbol{}
$$

In other words, $\widehat{u}\left(s_{i}\right)$ tends to $\widehat{u}(s)$ in the compact set $C \cdot f \boldsymbol{}$

$$
\widehat{u}\left(s_{i}\right)=u \cdot s_{i} \underset{i \rightarrow \infty}{\stackrel{C \cdot f}{\longrightarrow}} u \cdot s=\widehat{u}(s)
$$

Hence, $\widehat{u}\left(s_{i}\right)$ tends to $\widehat{u}(s)$ in the space $\mathcal{O}_{\exp }(G)$ :

$$
\widehat{u}\left(s_{i}\right) \underset{i \rightarrow \infty}{\stackrel{\mathcal{O}_{\exp }(G)}{\longrightarrow}} \widehat{u}(s)
$$

3. The continuity of the mapping $\widehat{u}: G \rightarrow \mathcal{O}_{\exp }(G)$ implies that for any functional $\beta \in \mathcal{O}_{\text {exp }}^{\star}(G)$ the function $\beta \circ \widehat{u}: G \rightarrow \mathbb{C}$ is holomorphic. We can use the Morera theorem to prove this: consider a 
closed oriented hypersurface $\Gamma$ in $G$ with a sufficiently small diameter so that integral over $\Gamma$ of every holomorphic function vanishes, and show that the integral of $\beta \circ \widehat{u}$ also vanishes:

$$
\int_{\Gamma}(\beta \circ \widehat{u})(s) \mathrm{d} s=0
$$

Indeed, take a net of functionals $\left\{\beta_{i} ; i \rightarrow \infty\right\} \subset \mathcal{O}_{\text {exp }}^{\star}(G)$ approximating $\beta$ in $\mathcal{O}_{\text {exp }}^{\star}(G)$, and having a form of linear combinations of delta-functionals:

$$
\beta_{i}=\sum_{k} \lambda_{i}^{k} \cdot \delta^{a_{i}^{k}}, \quad \beta_{i} \underset{i \rightarrow \infty}{\stackrel{\mathcal{O}_{\text {exp }}^{\star}(G)}{\longrightarrow}} \beta
$$

Then we obtain the following: since $\widehat{u}: G \rightarrow \mathcal{O}_{\exp }(G)$ is continuous,

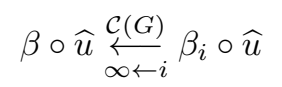

From this it follows that for any Radon measure $\alpha \in \mathcal{C}(G)$

$$
\alpha(\beta \circ \widehat{u}) \underset{\infty \leftarrow i}{\overleftarrow{\infty}} \alpha\left(\beta_{i} \circ \widehat{u}\right)
$$

In particular for the functional of integration over the hypersurface $\Gamma$ we have

$$
\begin{aligned}
\int_{\Gamma}(\beta \circ \widehat{u})(s) \mathrm{d} s \overleftarrow{\infty \leftarrow i} \int_{\Gamma}\left(\beta_{i} \circ \widehat{u}\right)(s) \mathrm{d} s=\int_{\Gamma}\left(\sum_{k} \lambda_{i}^{k} \cdot \delta^{a_{i}^{k}} \circ \widehat{u}\right)(s) \mathrm{d} s= \\
=\sum_{k} \lambda_{i}^{k} \cdot \int_{\Gamma}\left(\delta^{a_{i}^{k}} \circ \widehat{u}\right)(s) \mathrm{d} s=\sum_{k} \lambda_{i}^{k} \cdot \int_{\Gamma} \delta^{a_{i}^{k}}(\widehat{u}(s)) \mathrm{d} s= \\
=\sum_{k} \lambda_{i}^{k} \cdot \int_{\Gamma} \widehat{u}(s)\left(a_{i}^{k}\right) \mathrm{d} s=\sum_{k} \lambda_{i}^{k} \cdot \underbrace{\int_{\Gamma} u\left(s \cdot a_{i}^{k}\right) \mathrm{d} s}_{\begin{array}{l}
\|, \\
0, \\
\text { since } u \text { is holomorphic }
\end{array}}=0
\end{aligned}
$$

So indeed (4.21) is true.

4. We have showed that for any functional $\beta \in \mathcal{O}_{\text {exp }}^{\star}(G)$ the function $\beta \circ \widehat{u}: G \rightarrow \mathbb{C}$ is holomorphic. Let us show now that this function is of exponential type:

$$
\forall u \in \mathcal{O}_{\exp }(G) \quad \forall \beta \in \mathcal{O}_{\text {exp }}^{\star}(G) \quad \beta \circ \widehat{u} \in \mathcal{O}_{\exp }(G)
$$

Indeed, since the functional $\beta \in \mathcal{O}_{\text {exp }}^{\star}(G)$ is bounded on the compact set $f \subseteq \mathcal{O}_{\exp }(G)$, it is a bounded functional on the Banach representation of the Smith space $\mathbb{C} f$, i.e. the following inequality holds:

$$
\forall v \in \mathbb{C} f \quad|\beta(v)| \leqslant M \cdot\|v\|_{f} \text { ! }
$$

where

$$
M=\|\beta\|_{(f \mathbf{\downarrow})}:=\max _{v \in f}|\beta(v)|, \quad\|v\|_{f} \mathbf{\downarrow}:=\inf \{\lambda>0: v \in \lambda \cdot f\}
$$

Now from formula (4.20) we have

$$
\begin{aligned}
& \forall s \in G \quad u \cdot s \in f(s) \cdot f \quad \Longrightarrow \quad\|u \cdot s\|_{f}:=\inf \{\lambda>0: u \cdot s \in \lambda \cdot f \quad\} \leqslant f(s) \quad \Longrightarrow \\
& \Longrightarrow \quad|(\beta \circ \widehat{u})(s)|=|\beta(u \cdot s)| \leqslant M \cdot\|u \cdot s\|_{f} \leqslant M \cdot f(s)
\end{aligned}
$$

So the function $\beta \circ \widehat{u}$ is bounded by the semicharacter $M \cdot f=\|\beta\|_{(f \mathbf{\downarrow})} \cdot f$ :

$$
u \in f \quad \Longrightarrow \quad \beta \circ \widehat{u} \in\|\beta\|_{(f)^{\circ}} \cdot f \mathbf{\downarrow}
$$


5. Now let us note that

$$
(\beta \circ \widehat{u})(s)=\beta(u \cdot s)=(\underline{3.13})=(u * \widetilde{\beta})(s), \quad s \in G
$$

In other words, we proved that the function $u * \widetilde{\beta}=\beta \circ \widehat{u}$ belongs to the space $\mathcal{O}_{\exp }(G)$, so for any functional $\alpha \in \mathcal{O}_{\exp }^{\star}(G)$ the convolution

$$
\alpha * \beta(u)=(3.14)=\alpha(u * \tilde{\beta})
$$

is defined. It remains to verify that the operation $(\alpha, \beta) \mapsto \alpha * \beta$ is a continuous bilinear form, i.e. satisfies the conditions (i) and (ii) of Proposition 1.1.

Let $\alpha_{i}$ be a net tending to zero in $\mathcal{O}_{\exp }(G)$, and $B$ a compact set in $\mathcal{O}_{\text {exp }}^{\star}(G)$. Let us consider an arbitrary compact set $D$ in $\mathcal{O}_{\exp }(G)$. By Corollary 4.2, it must be contained in a rectangle $f$, where $f$ is a semicharacter:

$$
D \subseteq f^{\square}
$$

On the other hand, on the Smith space $\mathbb{C} f$ the norms of functionals $\beta \in B$ are bounded:

$$
\sup _{\beta \in B}\|v\|_{f} \mathbf{\square}=M<\infty
$$

So by virtue of (4.24) we obtain that

$$
\forall u \in D \quad \forall \beta \in B \quad u * \widetilde{\beta}=\beta \circ \widehat{u} \in\|\beta\|_{(f \mathbf{\varpi})} \cdot f \subseteq M \cdot f
$$

Thus the set $\{u * \widetilde{\beta} ; u \in D, \beta \in B\}$ is contained in the compact set $M \cdot f^{\mathbf{\varpi}}$ in the space $\mathcal{O}_{\text {exp }}^{\star}(G)$. Hence, the net of functionals $\alpha_{i}$ tends to zero uniformly on this set:

$$
\left(\alpha_{i} * \beta\right)(u)=\alpha_{i}(u * \widetilde{\beta}) \underset{i \rightarrow \infty}{\stackrel{\mathbb{C}}{\rightrightarrows}} 0 \quad(u \in D, \beta \in B)
$$

This is true for any compact set $D$, so the net $\alpha_{i} * \beta$ tends to zero in the space $\mathcal{O}_{\text {exp }}^{\star}(G)$ uniformly by $\beta \in B$ :

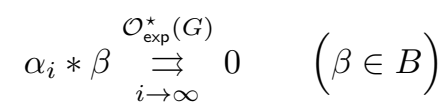

We can omit the case of the inverse sequence of multipliers due to the identity

$$
\widetilde{\alpha * \beta}=\widetilde{\beta} * \widetilde{\alpha}
$$

\section{(d) Examples.}

Finite groups. As we have noted in $\S 3$ (a) every finite group $G$, considered as a zero-dimensional complex manifold, is a linear complex Lie group on which every function is holomorphic. On the other hand, every function $u: G \rightarrow \mathbb{C}$ is bounded (since its set of values is finite), so $u$ must be a function of exponential type. Thus, algebras $\mathcal{O}_{\text {exp }}(G)$ and $\mathcal{O}(G)$ in this case coincide with each other and are equal to the algebra $\mathbb{C}^{G}$ of all functions on $G$ (with the pointwise algebraic operations and the topology of pointwise convergence):

$$
\mathcal{O}_{\exp }(G)=\mathcal{O}(G)=\mathbb{C}^{G}
$$


Groups $\mathbb{C}^{n}$. For the case of complex plane $\mathbb{C}$ our definition, certainly, coincide with the classical one functions of exponential type on $\mathbb{C}$ are entire functions $u \in \mathcal{O}(\mathbb{C})$ growing not faster than the exponential:

$$
\exists A>0: \quad|u(x)| \leqslant A^{|x|} \quad(x \in \mathbb{C}) .
$$

According to the classical theorems on the growth of entire functions [22, 33, this is equivalent to the condition that the derivatives of $u$ in a fixed point, say in zero, grow not faster than the exponential:

$$
\exists B>0: \quad\left|u^{(k)}(0)\right| \leqslant B^{k} \quad(k \in \mathbb{N}) .
$$

The same is true for several variables: functions of exponential type on $\mathbb{C}^{n}$ according to our definition will be exactly the functions $u \in \mathcal{O}\left(\mathbb{C}^{n}\right)$ satisfying the condition

$$
\exists A>0: \quad|u(x)| \leqslant A^{|x|} \quad\left(x \in \mathbb{C}^{n}\right),
$$

which turns out to be equivalent to the condition

$$
\exists B>0: \quad\left|u^{(k)}(0)\right| \leqslant B^{|k|} \quad\left(k \in \mathbb{N}^{n}\right),
$$

where the factorial $k$ ! and the modulus $|k|$ of a multiindex $k=\left(k_{1}, \ldots, k_{n}\right) \in \mathbb{N}^{n}$ are defined by the equalities

$$
k !:=k_{1} \cdot \ldots \cdot k_{n}, \quad|k|:=k_{1}+\ldots+k_{n}
$$

The equivalence of (4.25) to our definition follows from Proposition 4.2, and the equivalence between (4.25) and (4.26) is proved similarly with the case of one variable: the implication (4.26) $\Rightarrow(4.25)$ is obvious, and the inverse implication $(4.25) \Rightarrow(4.26)$ is a corollary of the Cauchy inequalities (see [34]) for the coefficients $c_{k}=\frac{u^{(k)}(0)}{k !}$ of the Taylor series for the function $u$ :

$$
\begin{gathered}
\forall R>0 \quad\left|c_{k}\right| \leqslant \frac{\max _{|x| \leqslant R}|u(x)|}{R^{|k|}} \leqslant(4.25) \leqslant \frac{A^{R}}{R^{|k|}} \\
\Downarrow \\
\left|c_{k}\right| \leqslant \min _{R>0} \frac{A^{R}}{R^{|k|}}=\left.\frac{A^{R}}{R^{|k|}}\right|_{R=\frac{|k|}{\log A}}=\frac{A^{\frac{|k|}{\log A}}}{|k|^{|k|}} \cdot(\log A)^{|k|}=\frac{1}{|k|^{|k|}} \cdot B^{|k|}, \quad B=A^{\frac{1}{\log A}} \cdot \log A \\
\Downarrow \quad k u^{(k)}(0)|=k ! \cdot| c_{k} \mid \leqslant \frac{k !}{|k|^{|k|}} \cdot B^{|k|} \leqslant B^{|k|}
\end{gathered}
$$

Groups $\mathrm{GL}_{n}(\mathbb{C})$. On the group $\mathrm{GL}_{n}(\mathbb{C})$ the functions of exponential type are exactly polynomials, i.e. the functions of the form

$$
u(x)=\frac{P(x)}{(\operatorname{det} x)^{m}}, \quad x \in \mathrm{GL}_{n}(\mathbb{C}), \quad m \in \mathbb{Z}_{+}
$$

where $P$ is a usual polynomial on matrix elements of the matrix $x$, and $\operatorname{det} x$ is its determinant. Thus, the equality holds:

$$
\mathcal{O}_{\exp }\left(\mathrm{GL}_{n}(\mathbb{C})\right)=\mathcal{R}\left(\mathrm{GL}_{n}(\mathbb{C})\right)
$$

Proof. 1. First, let us prove the inclusion $\mathcal{R}\left(\mathrm{GL}_{n}(\mathbb{C})\right) \subseteq \mathcal{O}_{\exp }\left(\mathrm{GL}_{n}(\mathbb{C})\right)$. Note for this that every matrix element

$$
x \mapsto x_{k, l}
$$


is a function of exponential type, since it is bounded by, for instance the first of the matrix norms in (4.5):

$$
\left|x_{k, l}\right| \leqslant \sum_{i, j=1}^{n}\left|x_{i, j}\right|=|| x \|
$$

As a corollary, every polynomial $x \mapsto P(x)$ of matrix elements is also a function of exponential type (since functions of exponential type form an algebra).

Further, every power of the determinant, $x \mapsto(\operatorname{det} x)^{-m}$, is multiplicative

$$
(\operatorname{det}(x \cdot y))^{-m}=(\operatorname{det} x)^{-m} \cdot(\operatorname{det} y)^{-m}
$$

so its absolute value is also multiplicative:

$$
\left|(\operatorname{det}(x \cdot y))^{-m}\right|=\left|(\operatorname{det} x)^{-m}\right| \cdot\left|(\operatorname{det} y)^{-m}\right|
$$

Therefore, the function $x \mapsto\left|(\operatorname{det} x)^{-m}\right|$ is a semicharacter on $\mathrm{GL}_{n}(\mathbb{C})$, and $x \mapsto(\operatorname{det} x)^{-m}$ a function of exponential type on $\mathrm{GL}_{n}(\mathbb{C})$.

Now, if we multiply two functions of exponential type $x \mapsto P(x)$ and $x \mapsto(\operatorname{det} x)^{-m}$, we obtain a function of exponential type $x \mapsto P(x) /(\operatorname{det} x)^{m}$.

2. Let us prove the inverse inclusion: $\mathcal{O}_{\exp }\left(\mathrm{GL}_{n}(\mathbb{C})\right) \subseteq \mathcal{R}\left(\mathrm{GL}_{n}(\mathbb{C})\right)$. If $u$ is a holomorphic function of exponential type on $\mathrm{GL}_{n}(\mathbb{C})$, then by Proposition 4.1 it must be bounded by some semicharacter of the form (4.6). In particular, for some $C \geqslant 1$ and $N \in \mathbb{N}$

$$
|u(x)| \leqslant C \cdot \max \left\{\|x\| ; \| x^{-1}||\right\}^{N}, \quad\|x\|=\sum_{i, j=1}^{n}\left|x_{i, j}\right|
$$

The elements of the inverse matrix $x^{-1}$ are obtained from $x$ as complementary minors, divided by the determinant, so we can treat them as polynomials (of degree $n-1)$ of $x_{i, j}$ and $(\operatorname{det} x)^{-1}$. This means that one can estimate the righthand side of (4.29) by a polynomial (of degree $N(n-1)$ ) of $\left|x_{i, j}\right|$ and $|\operatorname{det} x|^{-1}$ with nonnegative coefficients:

$$
|u(x)| \leqslant C \cdot \max \left\{\|x\| ; \| x^{-1}||\right\}^{N} \leqslant C \cdot P\left(\left\{\left|x_{i, j}\right|\right\}_{1 \leqslant i, j \leqslant n},|\operatorname{det} x|^{-1}\right)
$$

Hence if we multiply $u$ by $(\operatorname{det} x)^{N(n-1)}$, we obtain a holomorphic function on $\mathrm{GL}_{n}(\mathbb{C})$ bounded by a polynomial of $\left|x_{i, j}\right|$ :

$$
\left|u(x) \cdot(\operatorname{det} x)^{N(n-1)}\right| \leqslant C \cdot Q\left(\left\{\left|x_{i, j}\right|\right\}_{1 \leqslant i, j \leqslant n}\right)
$$

Such a function is locally bounded in the points of the analytical set $\operatorname{det} x=0$, so by the Riemann extension theorem (see [34]), it can be extended to a holomorphic function to the manifold $\mathrm{M}_{n}(\mathbb{C}$ ) (of all complex matrices). Therefore, we can consider $u(x) \cdot(\operatorname{det} x)^{N(n-1)}$ as a holomorphic function on $\mathrm{M}_{n}(\mathbb{C})=\mathbb{C}^{n^{2}}$. Since it has a polynomial growth, it must be a polynomial $q$ of matrix elements $x_{i, j}$ :

$$
u(x) \cdot(\operatorname{det} x)^{N(n-1)}=q(x)
$$

Hence

$$
u(x)=\frac{q(x)}{(\operatorname{det} x)^{N(n-1)}},
$$

i.e. $u \in \mathcal{R}\left(\mathrm{GL}_{n}(\mathbb{C})\right)$.

Corollary 4.3. On the complex circle $\mathbb{C}^{\times}$the functions of exponential type are exactly the Laurent polynomials:

$$
u(t)=\sum_{|n| \leqslant N} u_{n} \cdot t^{n}, \quad N \in \mathbb{N}
$$




\section{(e) Injection $b_{G}: \mathcal{O}_{\exp }(G) \rightarrow \mathcal{O}(G)$}

We need to denote the injection of $\mathcal{O}_{\exp }(G)$ into $\mathcal{O}(G)$ by some symbol. Let us use for this the symbol $b_{G}$ :

$$
b_{G}: \mathcal{O}_{\exp }(G) \rightarrow \mathcal{O}(G)
$$

This mapping is always injective, a homomorphism of algebras, and by definition of topology in $\mathcal{O}_{\exp }(G)$, always continuous. Below in Theorem 4.9 we shall see that if $G$ is a linear group, then $b_{G}$ has dense image in $\mathcal{O}(G)$.

From equality (4.28) and Theorem 4.6]it follows that if $G$ is an arbitrary linear group (with a given representation as a closed subgroup in $\mathrm{GL}_{n}(\mathbb{C})$ ), then every function $G$ that can be extended to a polynomial on $\mathrm{GL}_{n}(\mathbb{C})$, is a function of exponential type. Thus, we have the following chain of inclusions:

$$
\mathcal{R}(G) \subseteq \mathcal{O}_{\exp }(G) \subseteq \mathcal{O}(G)
$$

(here $\mathcal{R}(G)$ denotes the functions that can be extended to polynomials on $\mathrm{GL}_{n}(\mathbb{C})$ - we need this specification since $G$ is not necessarily an algebraic group).

Theorem 4.9. If $G$ is a linear complex group, then the algebra $\mathcal{O}_{\exp }(G)$ of holomorphic functions of exponential type on $G$ is dense in the algebra $\mathcal{O}(G)$ of all holomorphic functions on $G$.

Proof. Let $\varphi: G \rightarrow \mathrm{GL}_{n}(\mathbb{C})$ be a holomorphic embedding as a closed subgroup. By one of the corollaries from the Cartan theorem [38, 11.5.2], every holomorphic function $v \in \mathcal{O}(G)$ can be extended to a holomorphic function $u \in \mathcal{O}\left(\mathrm{GL}_{n}(\mathbb{C})\right)$. Let us approximate $u$ uniformly on compact set by polynomials $u_{i} \in \mathcal{R}\left(\mathrm{GL}_{n}(\mathbb{C})\right)$. By (4.28), all polynomials $u_{i}$ are functions of exponential type on $\mathrm{GL}_{n}(\mathbb{C})$, hence their restrictions $\left.u_{i}\right|_{G}$ are functions of exponential type on $G$. Thus, $v$ is approximated by functions of exponential type $\left.u_{i}\right|_{G}$ uniformly on compact sets in $G$.

\section{(f) Nuclearity of the spaces $\mathcal{O}_{\exp }(G)$ and $\mathcal{O}_{\text {exp }}^{\star}(G)$}

Theorem 4.10. For any compactly generated Stein group $G$ the space $\mathcal{O}_{\exp }(G)$ is a nuclear Brauner space, and its dual space $\mathcal{O}_{\text {exp }}^{\star}(G)$ a nuclear Fréchet space.

We premise the proof of this fact by two lemmas. The first of them is true for arbitrary complex manifold and is proved by the same technique that is applied for the proof of nuclearity of $\mathcal{O}(\mathbb{C})$ (see [25, 6.4.2]):

Lemma 4.3. If $M$ is a complex manifold, and $K$ and $L$ are two compact sets in $M$, such that $K$ is strictly contained in $L$,

$$
K \subseteq \operatorname{lnt} L
$$

then there exists a constant $C \geqslant 0$ and a probability measure $\mu$ on $L$ such that for any $u \in \mathcal{O}(G)$ we have

$$
|u|_{K} \leqslant C \cdot \int_{L}|\alpha(u)| \mu(\mathrm{d} \alpha)
$$

As a corollary, the operator $\left.\left.u\right|_{L} \mapsto u\right|_{K}$ of restriction is absolutely summing, and its quasinorm of absolute summing is not greater than $C$ : for any $u_{1}, \ldots, u_{l} \in \mathcal{O}(M)$

$$
\sum_{i=1}^{l}\left|u_{i}\right|_{K} \leqslant C \cdot \int_{L} \sum_{i=1}^{l}\left|\alpha\left(u_{i}\right)\right| \mu(\mathrm{d} \alpha) \leqslant C \cdot \sum_{\alpha \in \operatorname{\operatorname {absconv}}\left(\delta^{L}\right)} \sum_{i=1}^{l}\left|\alpha\left(u_{i}\right)\right|
$$

Remark 4.1. Here $\overline{\text { absconv }}\left(\delta^{L}\right)$ means the universal compact set in the Smith space $\mathcal{C}^{\star}(L)$ dual to the Banach space $\mathcal{C}(L)$ of continuous functions on $L$, or what is the same, the unit ball in the Banach space $\mathcal{M}(L)$ of Radon measures on $L$. We use this notation, because it is convenient to denote by 
$\delta^{L}=\left\{\delta^{x} ; x \in L\right\}$ the set of all delta-functionals on $\mathcal{C}(L)$ - then the polar $B^{\circ}$ of the unit ball $B$ in $\mathcal{C}(L)$ coincides with the absolutely convex hull of $\delta^{L}$ :

$$
B^{\circ}=\overline{\operatorname{absconv}}\left(\delta^{L}\right)=\{\alpha \in M(L):\|\alpha\| \leqslant 1\}
$$

(the closure with respect to topology of $\mathcal{C}^{\star}(L)$ ).

Lemma 4.4. For any generating compact neighborhood of identity $K$ in $G$

$$
G=\bigcup_{n=1}^{\infty} K^{n}
$$

there are constants $C \geqslant 0, \lambda \geqslant 0$ such that for any $l \in \mathbb{N}$ and for arbitrary $u_{1}, \ldots, u_{l} \in \mathcal{O}(G), n \in \mathbb{N}$ the following inequality holds:

$$
\sum_{i=1}^{l}\left|u_{i}\right|_{K^{n}} \leqslant C \cdot \lambda^{n-1} \cdot \sup _{\alpha \in \operatorname{absconv}\left(\delta^{K^{2 n+1}}\right)} \sum_{i=1}^{l}\left|\alpha\left(u_{i}\right)\right|
$$

Proof. 1. The set $U=\operatorname{lnt} K$ is an open neighborhood of identity in $G$, so the system of shifts $\{x \cdot U ; x \in$ $\left.K^{2}\right\}$ is an open covering of the compact set $K^{2}$. Let us choose a finite subcovering, i.e. a finite set $F \subseteq K^{2}$ such that

$$
K^{2} \subseteq \bigcup_{x \in F} x \cdot U=F \cdot U
$$

Then we obtain:

$$
K^{n} \subseteq F^{n-1} \cdot K \subseteq F^{n-1} \cdot K^{2} \subseteq K^{2 n+1}, \quad n \in \mathbb{N}
$$

Here is the proof: first we should note that

$$
F \subseteq K^{2} \quad \Longrightarrow \quad F^{n} \subseteq K^{2 n}, \quad n \in \mathbb{N}
$$

After that in the sequence (4.34) it is sufficient to check only the first inclusion:

$$
K^{n} \subseteq F^{n-1} \cdot K, \quad n \in \mathbb{N}
$$

This is done by the induction: for $n=2$ we have

$$
K^{2} \subseteq F \cdot U \subseteq F \cdot K
$$

and, if (4.35) is true for some $n$, then for $n+1$ we have:

$$
K^{n+1}=K^{n} \cdot K \subseteq F^{n-1} \cdot K \cdot K \subseteq F^{n-1} \cdot K^{2} \subseteq F^{n-1} \cdot F \cdot K=F^{n} \cdot K
$$

2. Since the compact set $K^{2}$ strictly contains $K$, by Lemma 4.3 there are $C, \mu$ such that

$$
|u|_{K} \leqslant C \cdot \int_{K^{2}}|\alpha(u)| \mu(\mathrm{d} \alpha)
$$

Under the shift by an element $x \in G$ this inequality takes form

$$
|u|_{x \cdot K} \leqslant C \cdot \int_{x \cdot K^{2}}|\alpha(u)|(x \cdot \mu)(\mathrm{d} \alpha)
$$

where $x \cdot \mu$ is a shift of the measure $\mu$ :

$$
(x \cdot \mu)(u)=\mu(u \cdot x), \quad(u \cdot x)(t)=u(x \cdot t)
$$


This implies that if $E$ is an arbitrary finite set in $G$, then the sum

$$
\nu=\frac{1}{\operatorname{card} E} \sum_{x \in E} x \cdot \mu
$$

is a probability measure on the set $E \cdot K^{2}$, with the property

$$
\begin{aligned}
& |u|_{E \cdot K} \leqslant \sum_{x \in E}|u|_{x \cdot K} \leqslant C \cdot \sum_{x \in E} \int_{x \cdot K^{2}}|\alpha(u)|(x \cdot \mu)(\mathrm{d} \alpha)= \\
& =C \cdot \sum_{x \in E} \int_{E \cdot K^{2}}|\alpha(u)|(x \cdot \mu)(\mathrm{d} \alpha)=C \cdot \int_{E \cdot K^{2}}|\alpha(u)|\left(\sum_{x \in E} x \cdot \mu\right)(\mathrm{d} \alpha)= \\
& =C \cdot \operatorname{card}(E) \cdot \int_{E \cdot K^{2}}|\alpha(u)| \nu(\mathrm{d} \alpha)
\end{aligned}
$$

From this we deduce that the restriction operator $\left.\left.u\right|_{E \cdot K^{2}} \mapsto u\right|_{E \cdot K}$ is absolutely summing, and the quasinorm of absolute summing can be estimated by the constant $C \cdot \operatorname{card}(E)$ : for all $u_{1}, \ldots, u_{l} \in \mathcal{O}(G)$

$$
\sum_{i=1}^{l}\left|u_{i}\right|_{E \cdot K} \leqslant C \cdot \operatorname{card}(E) \cdot \int_{E \cdot K^{2}} \sum_{i=1}^{l}\left|\alpha\left(u_{i}\right)\right| \nu(\mathrm{d} \alpha) \leqslant C \cdot \operatorname{card}(E) \cdot \sup _{\alpha \in \operatorname{absconv}\left(\delta^{E} \cdot K^{2}\right.} \sum_{i=1}^{l}\left|\alpha\left(u_{i}\right)\right|
$$

Now from formulas (4.34) we have:

$$
\begin{aligned}
& \sum_{i=1}^{l}\left|u_{i}\right|_{K^{n}} \leqslant \sum_{i=1}^{l}\left|u_{i}\right|_{F^{n-1} \cdot K} \leqslant C \cdot \operatorname{card}\left(F^{n-1}\right) \cdot \sup _{\alpha \in \overline{\operatorname{absconv}}\left(\delta^{F^{n-1} \cdot K^{2}}\right)} \sum_{i=1}^{l}\left|\alpha\left(u_{i}\right)\right| \leqslant \\
& \leqslant C \cdot \operatorname{card}\left(F^{n-1}\right) \cdot \sum_{\alpha \in \operatorname{a\operatorname {abconv}}\left(\delta^{K^{2 n+1}}\right)} \sum_{i=1}^{l}\left|\alpha\left(u_{i}\right)\right| \leqslant C \cdot(\operatorname{card}(F))^{n-1} \cdot \sum_{\alpha \in \overline{\operatorname{absconv}}\left(\delta^{K^{2 n+1}}\right)} \sum_{i=1}^{l}\left|\alpha\left(u_{i}\right)\right|
\end{aligned}
$$

To obtain (4.33) we can put $\lambda=\operatorname{card} F$.

Proof of Theorem 4.10. Consider the sequence of rectangles as in Theorem 4.3

$$
E_{N}=\left(f_{N}\right)
$$

By Theorem 4.3, their union covers the whole space $\mathcal{O}_{\exp }(G)$

$$
\mathcal{O}_{\exp }(G)=\bigcup_{N=1}^{\infty} E_{N}
$$

and moreover any dually submultiplicative rectangle $D \subseteq \mathcal{O}(G)$ is contained in some rectangle $E_{N}$ :

$$
D \subseteq E_{N}
$$

From this we deduce that in Formulas (4.16) and (4.17) the system of all dually submultiplicative rectangles $D$ can be replaced by the system of rectangles $E_{N}$ :

$$
\mathcal{O}_{\exp }(G)=\bigcup_{N=1}^{\infty} \mathbb{C} E_{N}=\lim _{\substack{\rightarrow \rightarrow \infty \\ N \rightarrow \infty}} \mathbb{C} E_{N}
$$

This means that $\mathcal{O}_{\exp }(G)$ is a Brauner space, and $\mathcal{O}_{\text {exp }}^{\star}(G)$ a Fréchet space. To prove that both spaces are nuclear we can only note that $\mathcal{O}_{\exp }(G)$ is conuclear. Consider $\mathbb{C} E_{N}$ as Banach spaces with unit balls $E_{N}$. By (4.14), the seminorm on $\mathbb{C} E_{N}$ is defined by equality

$$
p_{N}(u)=\sup _{n \in \mathbb{N}} \frac{1}{N^{n}}|u|_{K^{n}}
$$


Its unit ball is the set $E_{N}$ :

$$
\begin{aligned}
E_{N}=\left\{u \in \mathcal{O}(G): \forall n \in \mathbb{N} \quad|u|_{K^{n}} \leqslant N^{n}\right\}=\left\{u \in \mathcal{O}(G): \forall n \in \mathbb{N} \quad \frac{1}{N^{n}}|u|_{K^{n}} \leqslant 1\right\}= \\
=\left\{u \in \mathcal{O}(G): p_{N}(u)=\sup _{n \in \mathbb{N}} \frac{1}{N^{n}}|u|_{K^{n}} \leqslant 1\right\}
\end{aligned}
$$

To prove that the space $\mathcal{O}_{\exp }(G)=\lim _{\substack{\rightarrow \rightarrow \infty \\ \rightarrow}} \mathbb{C} E_{N}$ is conuclear, we need to verify that for any $N \in \mathbb{N}$ there exists $M \in \mathbb{N}, M>N$, such that the inclusion mapping $\mathbb{C} E_{N} \rightarrow \mathbb{C} E_{M}$ is absolutely summing, i.e. such that for some constant $L>0$, for any $l \in \mathbb{N}$ and for all $u_{1}, \ldots, u_{l} \in \mathbb{C} E_{N}$ we have:

$$
\sum_{i=1}^{l} p_{M}\left(u_{i}\right) \leqslant L \cdot \sup _{\alpha \in\left(E_{N}\right)^{\circ}} \sum_{i=1}^{l}\left|\alpha\left(u_{i}\right)\right|
$$

This is proved as follows. First we need to note that for all $n, N \in \mathbb{N}$ the following inclusion holds

$$
E_{N}=\left\{u \in \mathcal{O}(G): \forall n \in \mathbb{N} \quad|u|_{K^{n}} \leqslant N^{n}\right\} \subseteq\left\{u \in \mathcal{O}(G):|u|_{K^{n}} \leqslant N^{n}\right\}=N^{n} \cdot{ }^{\circ} \delta^{K^{n}}
$$

It implies following chain:

$$
\begin{aligned}
& E_{N} \subseteq N^{n} \cdot \circ\left(\delta^{K^{n}}\right) \\
& \Downarrow \\
& \left(E_{N}\right)^{\circ} \supseteq\left(N^{n} \cdot{ }^{\circ}\left(\delta^{K^{n}}\right)\right)^{\circ}=\frac{1}{N^{n}} \cdot\left({ }^{\circ}\left(\delta^{K^{n}}\right)\right)^{\circ}=\frac{1}{N^{n}} \cdot \overline{\operatorname{absconv}}\left(\delta^{K^{n}}\right) \\
& \Downarrow \\
& \overline{\operatorname{absconv}}\left(\delta^{K^{n}}\right) \subseteq N^{n} \cdot\left(E_{N}\right)^{\circ} \\
& \Downarrow \\
& \overline{\operatorname{absconv}}\left(\delta^{K^{2 n+1}}\right) \subseteq N^{2 n+1} \cdot\left(E_{N}\right)^{\circ} \\
& \Downarrow \\
& \sum_{i=1}^{l}\left|u_{i}\right|_{K^{n}} \leqslant(4.33) \leqslant C \cdot \lambda^{n-1} \cdot \sup _{\alpha \in \operatorname{absconv}\left(\delta^{K^{2 n+1}}\right)} \sum_{i=1}^{l}\left|\alpha\left(u_{i}\right)\right| \leqslant C \cdot \lambda^{n-1} \cdot \sup _{\alpha \in N^{2 n+1} \cdot\left(E_{N}\right)^{\circ}} \sum_{i=1}^{l}\left|\alpha\left(u_{i}\right)\right|= \\
& =C \cdot \lambda^{n-1} \cdot \sup _{\beta \in\left(E_{N}\right)^{\circ}} \sum_{i=1}^{l}\left|N^{2 n+1} \cdot \beta\left(u_{i}\right)\right|=C \cdot \lambda^{n-1} \cdot N^{2 n+1} \cdot \sup _{\beta \in\left(E_{N}\right)^{\circ}} \sum_{i=1}^{l}\left|\beta\left(u_{i}\right)\right| \\
& \Downarrow \\
& \forall M>0 \quad \sum_{i=1}^{l} \frac{1}{M^{n}}\left|u_{i}\right|_{K^{n}} \leqslant C \cdot \frac{\lambda^{n-1} \cdot N^{2 n+1}}{M^{n}} \cdot \sup _{\beta \in\left(E_{N}\right)^{\circ}} \sum_{i=1}^{l}\left|\beta\left(u_{i}\right)\right| \\
& \Downarrow \\
& \sum_{i=1}^{l} p_{M}\left(u_{i}\right)=\sum_{i=1}^{l} \sup _{n \in \mathbb{N}} \frac{1}{M^{n}}\left|u_{i}\right|_{K^{n}} \leqslant \sum_{i=1}^{l}\left(\sum_{n=1}^{\infty} \frac{1}{M^{n}}\left|u_{i}\right|_{K^{n}}\right)=\sum_{n=1}^{\infty}\left(\sum_{i=1}^{l} \frac{1}{M^{n}}\left|u_{i}\right|_{K^{n}}\right) \leqslant \\
& \leqslant \sum_{n=1}^{\infty}\left(C \cdot \frac{\lambda^{n-1} \cdot N^{2 n+1}}{M^{n}} \cdot \sup _{\beta \in\left(E_{N}\right)^{\circ}} \sum_{i=1}^{l}\left|\beta\left(u_{i}\right)\right|\right)=C \cdot\left(\sum_{n=1}^{\infty} \frac{\lambda^{n-1} \cdot N^{2 n+1}}{M^{n}}\right) \cdot \sup _{\beta \in\left(E_{N}\right)^{\circ}} \sum_{i=1}^{l}\left|\beta\left(u_{i}\right)\right|
\end{aligned}
$$


If we now choose $M \in \mathbb{N}$ sufficiently large such that

$$
C \cdot \sum_{n=1}^{\infty} \frac{\lambda^{n-1} \cdot N^{2 n+1}}{M^{n}} \leqslant 1
$$

then the constant $L$ in (4.38) can be chosen as 1 :

$$
\sum_{i=1}^{l} p_{M}\left(u_{i}\right) \leqslant \underbrace{C \cdot \sum_{n=1}^{\infty} \frac{\lambda^{n-1} \cdot N^{2 n+1}}{M^{n}}}_{\substack{\wedge \\ 1}} \cdot \sup _{\beta \in\left(E_{N}\right)^{\circ}} \sum_{i=1}^{l}\left|\beta\left(u_{i}\right)\right|
$$

\section{(g) Holomorphic mappings of exponential type and tensor products of the spaces $\mathcal{O}_{\exp }(G)$ and $\mathcal{O}_{\text {exp }}^{\star}(G)$}

Theorem 4.11. Let $G$ and $H$ be two compactly generated Stein groups. The formula

$$
\rho_{G, H}(u \boxminus v)=u \odot v, \quad u \in \mathcal{O}_{\exp }(G), \quad v \in \mathcal{O}_{\exp }(H)
$$

(where $u \boxminus v$ is the function from (1.24) ) defines a linear continuous mapping

$$
\rho_{G, H}: \mathcal{O}_{\exp }(G \times H) \rightarrow \mathcal{O}_{\exp }(G) \oslash \mathcal{O}_{\exp }^{\star}(H)=\mathcal{O}_{\exp }(G) \odot \mathcal{O}_{\exp }(H),
$$

This mapping is an isomorphism of stereotype spaces and is natural by $G$ and $H$, i.e. is an isomorphism of bifunctors from the category $\mathfrak{S} \mathfrak{G}$ of Stein groups into the category $\mathfrak{S t e}$ of stereotype spaces:

$$
((G ; H) \mapsto \mathcal{O}(G \times H)) \longmapsto((G ; H) \mapsto \mathcal{O}(G) \odot \mathcal{O}(H))
$$

Equivalently this mapping is defined by formula

$$
\rho_{G, H}(w)(\beta)=\beta \circ \widehat{w}, \quad w \in \mathcal{O}_{\exp }(G \times H), \quad \beta \in \mathcal{O}_{\exp }^{\star}(H)
$$

where

$$
\widehat{w}: G \rightarrow \mathcal{O}_{\exp }(H) \quad \widehat{w}(s)(t)=w(s, t), \quad s \in G, t \in H
$$

Corollary 4.4. The following isomorphisms of functors hold:

$$
\begin{aligned}
& \mathcal{O}_{\exp }(G \times H) \cong \mathcal{O}_{\exp }(G) \odot \mathcal{O}_{\exp }(H) \cong \mathcal{O}_{\exp }(G) \circledast \mathcal{O}_{\exp }(H) \\
& \mathcal{O}_{\exp }^{\star}(G \times H) \cong \mathcal{O}_{\exp }^{\star}(G) \odot \mathcal{O}_{\exp }^{\star}(H) \cong \mathcal{O}_{\exp }^{\star}(G) \circledast \mathcal{O}_{\exp }^{\star}(H)
\end{aligned}
$$

To prove Theorem 4.11 we have to recall the notion of injective tensor product $A \odot B$ of sets $A$ and $B$ in stereotype spaces $X$ and $Y$. According to notations [1, (7.27)], $A \odot B$ is defined as a subset in the space $X \odot Y=X \oslash Y^{\star}$ of operators $\varphi: Y^{\star} \rightarrow X$ containing only those operators satisfying the condition $\varphi\left(B^{\circ}\right) \subseteq A$. That is the sense of the following formula we use in Lemma 4.5 below:

$$
A \odot B=A \oslash B^{\circ} .
$$

Lemma 4.5. If $g: G \rightarrow \mathbb{R}_{+}$and $h: H \rightarrow \mathbb{R}_{+}$are two semicharacters on $G$ and $H$, then $g \square h$ is a semicharacter on $G \times H$, and the mapping $\rho_{G, H}$ defined in (4.41)-(4.42) is a homeomorphism between compact sets $(g \square h) \subseteq \mathcal{O}_{\exp }(G \times H)$ and $g \bullet h \subseteq \mathcal{O}_{\exp }(G) \odot \mathcal{O}_{\exp }(H)$ :

$$
(g \boxminus h) \cong g \bullet h^{\mathbf{\square}}
$$


Proof. Here at the beginning we use reasonings similar to those we used in the proof of Theorem 4.8

1. Note first that for any function $w \in(g \sqcup h) \subseteq \mathcal{O}_{\exp }(G \times H)$ Formula (4.42) defines some mapping

$$
\widehat{w}: G \rightarrow \mathcal{O}_{\exp }(H)
$$

Indeed, since $w$ is holomorphic on $G \times H$, it is holomorphic with respect to each of two variables, so when $s \in G$ is fixed, then the function $\widehat{w}(s): H \rightarrow \mathbb{C}$ is also holomorphic. At the same time it is bounded by the semicharacter $g(s) \cdot h$ :

$$
\forall s \in G \quad \widehat{w}(s) \in g(s) \cdot h^{\mathbf{}}
$$

since

$$
w \in(g \square h) \quad \Longrightarrow \quad|\widehat{w}(s)(t)|=|w(s, t)| \leqslant g(s) \cdot h(t) \quad \Longrightarrow \quad \widehat{w}(s) \in g(s) \cdot h
$$

Thus, $\widehat{w}(s)$ is always a holomorphic function of exponential type on $H$, i.e. $\widehat{w}\left((s) \in \mathcal{O}_{\exp }(H)\right.$

2. Let us show that the mapping $\widehat{w}: G \rightarrow \mathcal{O}_{\exp }(H)$ is continuous. Let $s_{i}$ be a sequence of points in $G$ tending to a point $s$ :

$$
s_{i} \underset{i \rightarrow \infty}{\stackrel{G}{\longrightarrow}} s
$$

Then the sequence of holomorphic functions $\widehat{w}\left(s_{i}\right) \in \mathcal{O}(H)$ tends to the holomorphic function $\widehat{w}(s) \in$ $\mathcal{O}(H)$ uniformly on each compact set $K \subseteq H$, i.e. in the space $\mathcal{O}(H)$ :

$$
\widehat{w}\left(s_{i}\right) \underset{i \rightarrow \infty}{\stackrel{\mathcal{O}(H)}{\longrightarrow}} \widehat{w}(s)
$$

On the other hand, all functions are bounded by the semicharacter $C \cdot h$, where $C=\max \left\{\sup _{i} g\left(s_{i}\right), g(s)\right\}$ is a finite number, since the sequence $s_{i}$ converges and together with its limit is a compact set:

$$
\left|\widehat{w}\left(s_{i}\right)(t)\right| \leqslant g\left(s_{i}\right) \cdot h(t) \leqslant C \cdot h(t)
$$

Thus, the functions $\widehat{w}\left(s_{i}\right)$ and $\widehat{w}(s)$ lie in a rectangle generated by the semicharacter $C \cdot h$ :

$$
\left\{\widehat{w}\left(s_{i}\right) ; \widehat{w}(s)\right\} \subseteq(C \cdot h)
$$

In other words, $\widehat{w}\left(s_{i}\right)$ tends to $\widehat{w}(s)$ on the compact set $(C \cdot h)$

$$
\widehat{w}\left(s_{i}\right) \underset{i \rightarrow \infty}{\stackrel{(C \cdot h)}{\longrightarrow}} \widehat{w}(s)
$$

so $\widehat{w}\left(s_{i}\right)$ tends to $\widehat{w}(s)$ in the space $\mathcal{O}_{\exp }(H)$ :

$$
\widehat{w}\left(s_{i}\right) \underset{i \rightarrow \infty}{\stackrel{\mathcal{O}_{\exp }(H)}{\longrightarrow}} \widehat{w}(s)
$$

3. From continuity of the mapping $\widehat{w}: G \rightarrow \mathcal{O}_{\exp }(H)$ it follows that for any functional $\beta \in \mathcal{O}_{\text {exp }}^{\star}(H)$ the function $\beta \circ \widehat{w}: G \rightarrow \mathbb{C}$ is holomorphic. To prove this one can use the Morera theorem: consider a closed oriented hypersurface $\Gamma$ in $G$ of dimension $n=\operatorname{dim} G$ with a sufficiently little diameter and show that

$$
\int_{\Gamma}(\beta \circ \widehat{w})(s) \mathrm{d} s=0
$$

Indeed, take a net of functionals $\left\{\beta_{i} ; i \rightarrow \infty\right\} \subset \mathcal{O}_{\text {exp }}^{\star}(H)$ which are linear combinations of deltafunctionals, and approximate $\beta$ in $\mathcal{O}_{\exp }^{\star}(H)$ :

$$
\beta_{i}=\sum_{k} \lambda_{i}^{k} \cdot \delta^{a_{i}^{k}}, \quad \beta_{i} \underset{i \rightarrow \infty}{\stackrel{\mathcal{O}_{\text {exp }}^{\star}(H)}{\longrightarrow}} \beta
$$

Then we obtain the following. Since $\widehat{w}: G \rightarrow \mathcal{O}_{\exp }(H)$ is continuous, we have

$$
\beta \circ \widehat{w} \underset{\infty \leftarrow i}{\stackrel{\mathcal{C}(G)}{\longleftarrow}} \beta_{i} \circ \widehat{w}
$$


This implies that for any Radon measure $\alpha \in \mathcal{C}(G)$

$$
\alpha(\beta \circ \widehat{w}) \underset{\infty \leftarrow i}{\longleftarrow} \alpha\left(\beta_{i} \circ \widehat{w}\right)
$$

In particular, for the functional of integrating by our hypersurface $\Gamma$ we obtain

$$
\begin{aligned}
& \int_{\Gamma}(\beta \circ \widehat{w})(s) \mathrm{d} s \underset{\infty \leftarrow i}{\overleftarrow{\infty}} \int_{\Gamma}\left(\beta_{i} \circ \widehat{w}\right)(s) \mathrm{d} s=\int_{\Gamma}\left(\sum_{k} \lambda_{i}^{k} \cdot \delta^{a_{i}^{k}} \circ \widehat{w}\right)(s) \mathrm{d} s= \\
& =\sum_{k} \lambda_{i}^{k} \cdot \int_{\Gamma}\left(\delta^{a_{i}^{k}} \circ \widehat{w}\right)(s) \mathrm{d} s=\sum_{k} \lambda_{i}^{k} \cdot \int_{\Gamma} \delta^{a_{i}^{k}}(\widehat{w}(s)) \mathrm{d} s= \\
& =\sum_{k} \lambda_{i}^{k} \cdot \int_{\Gamma} \widehat{w}(s)\left(a_{i}^{k}\right) \mathrm{d} s=\sum_{k} \lambda_{i}^{k} \cdot \underbrace{\int_{\Gamma} w\left(s, a_{i}^{k}\right) \mathrm{d} s}_{\begin{array}{c}
\| \\
0, \\
\text { since } w \text { is holomorphic }
\end{array}}=0 \\
& \text { with respect to the first variable }
\end{aligned}
$$

I.e., indeed (4.47) is true.

4. We understood that for any functional $\beta \in \mathcal{O}_{\exp }^{\star}(H)$ the function $\beta \circ \widehat{w}: G \rightarrow \mathbb{C}$ is holomorphic. Let us show now that it is of exponential type:

$$
\forall w \in \mathcal{O}_{\exp }(G \times H) \quad \forall \beta \in \mathcal{O}_{\exp }^{\star}(H) \quad \beta \circ \widehat{w} \in \mathcal{O}_{\exp }(G)
$$

Indeed, since the functional $\beta \in \mathcal{O}_{\text {exp }}^{\star}(H)$ is bounded on the compact set $h \subseteq \mathcal{O}_{\exp }(H)$, it must be a bounded functional on the Banach representation of the Smith space $\mathbb{C} h$, i.e.

$$
\forall v \in \mathbb{C} h^{\mathbf{\square}} \quad|\beta(v)| \leqslant M \cdot\|v\|_{h} \mathbf{\bullet}
$$

where

$$
M=\|\beta\|_{(h \mathbf{\square})}:=\max _{v \in h}|\beta(v)|, \quad\|v\|_{h} \mathbf{\square}:=\inf \left\{\lambda>0: v \in \lambda \cdot h^{\mathbf{\square}}\right\}
$$

So from formula (4.46) we have:

$$
\begin{aligned}
\widehat{w}(s) \in g(s) \cdot h \quad \Longrightarrow\|\widehat{w}(s)\|_{h}:=\inf \{\lambda>0: \widehat{w}(s) \in \lambda \cdot h\} \leqslant g(s) & \Longrightarrow \\
& \Longrightarrow|\beta(\widehat{w}(s))| \leqslant M \cdot g(s)
\end{aligned}
$$

I.e. the function $\beta \circ \widehat{w}$ is bounded by the semicharacter $M \cdot g$ :

$$
\beta \circ \widehat{w} \in M \cdot g
$$

5. We have proved (4.48). Now let us show that for any $w \in(g \boxminus h) \subseteq \mathcal{O}_{\exp }(G \times H)$ the mapping

$$
\beta \in \mathcal{O}_{\exp }^{\star}(H) \mapsto \rho_{G, H}(w)(\beta)=\beta \circ \widehat{w} \in \mathcal{O}_{\exp }(G)
$$

is continuous, i.e.

$$
\rho_{G, H}(w) \in \mathcal{O}_{\exp }(G) \oslash \mathcal{O}_{\exp }^{\star}(H)
$$

This follows from (4.50): if $\beta_{i}$ is a net tending to zero in $\mathcal{O}_{\exp }^{\star}(H)$, then

$$
\begin{gathered}
\beta_{i} \circ \widehat{w} \in M_{i} \cdot g \text { 】 } \quad M_{i}=\max _{v \in h}\left|\beta_{i}(v)\right| \underset{i \rightarrow \infty}{\longrightarrow} 0 \\
\Downarrow \\
\beta_{i} \circ \widehat{w} \underset{i \rightarrow \infty}{\stackrel{g}{\longrightarrow}} 0
\end{gathered}
$$




$$
\beta_{i} \circ \widehat{w} \underset{i \rightarrow \infty}{\stackrel{\mathcal{O}_{\exp }(G)}{\longrightarrow}} 0
$$

6. Now we need to verify that

$$
\rho_{G, H}(w) \in g^{\mathbf{\square}} \odot h^{\mathbf{\square}}=g^{\mathbf{\square}} \oslash\left(h^{\mathbf{\square}}\right)^{\circ} \subseteq \mathcal{O}_{\exp }(G) \oslash \mathcal{O}_{\exp }^{\star}(H)
$$

Or, in other words,

$$
\rho_{G, H}(w)\left(\left(h^{\mathbf{\bullet}}\right)^{\circ}\right) \subseteq g^{\mathbf{\square}}
$$

This follows from (4.46):

$$
\begin{aligned}
& \forall s \in G \quad \widehat{w}(s) \in g(s) \cdot h^{\mathbf{\square}} \\
& \Downarrow \\
& \forall s \in G \quad \frac{1}{g(s)} \widehat{w}(s) \in h^{\mathbf{W}} \\
& \Downarrow \\
& \forall s \in G \quad \forall \beta \in\left(h^{\mathbf{W}}\right)^{\circ} \quad 1 \geqslant\left|\beta\left(\frac{1}{g(s)} \widehat{w}(s)\right)\right|=\frac{1}{g(s)}|(\beta \circ \widehat{w})(s)|=\frac{1}{g(s)}\left|\left(\rho_{G, H}(w)(\beta)\right)(s)\right| \\
& \forall s \in G \quad \forall \beta \in\left(h^{\mathbf{\square}}\right)^{\circ} \quad\left|\rho_{G, H}(w)(\beta)(s)\right| \leqslant g(s) \\
& \Downarrow \\
& \forall \beta \in\left(h^{\mathbf{\varpi}}\right)^{\circ} \quad \rho_{G, H}(w)(\beta) \in g^{\mathbf{\square}} \\
& \Downarrow \\
& \rho_{G, H}(w)\left(\left(h^{\mathbf{\square}}\right)^{\circ}\right) \subseteq g
\end{aligned}
$$

7. Let us show that the mapping

$$
w \in(g \boxminus h)^{\mathbf{\square}} \mapsto \rho_{G, H}(w) \in g \text { 】 } \odot h^{\mathbf{\varpi}}
$$

is injective. Consider functionals of the form

$$
\delta^{s, t}: \mathcal{O}_{\exp }(G) \oslash \mathcal{O}_{\exp }^{\star}(H) \rightarrow \mathbb{C} \quad \delta^{s, t}(\varphi)=\varphi\left(\delta^{t}\right)(s)
$$

Now we have: if $w \neq 0$, then for some $s \in G, t \in H$ we have $w(s, t) \neq 0$, so

$$
\delta^{s, t}\left(\rho_{G, H}(w)\right)=\rho_{G, H}(w)\left(\delta^{t}\right)(s)=\left(\delta^{t} \circ \widehat{w}\right)(s)=\delta^{t}(\widehat{w}(s))=\widehat{w}(s)(t)=w(s, t) \neq 0
$$

thus, $\rho_{G, H}(w) \neq 0$.

8. Similarly it turns out that the mapping

$$
w \in(g \boxminus h)^{\mathbf{\square}} \mapsto \rho_{G, H}(w) \in g^{\mathbf{\square}} \odot h^{\mathbf{\square}}
$$

is surjective: for any $\varphi \in g \mathbf{\mathbf { \square }} \odot h^{\mathbf{\square}}=g^{\mathbf{\square}} \oslash(h \mathbf{\nabla})^{\circ} \subset \mathcal{O}_{\exp }(G) \oslash \mathcal{O}_{\text {exp }}^{\star}(H)$ we set

$$
w(s, t)=\delta^{s} \circledast \delta^{t}(\varphi)=\varphi\left(\delta^{t}\right)(s), \quad s \in G, t \in H
$$

and then, first, $w$ is a holomorphic function on $G \times H$, since it is holomorphic with respect to every variable: when $t \in H$ is fixed, the object $\varphi\left(\delta^{t}\right)$ is an element of the space $\mathcal{O}_{\exp }(G)$, i.e. a holomorphic function (of exponential type) on $G$, hence $w(\cdot, t)$ is holomorphic with respect to the first variable, and 
when $s \in G$ is fixed, the mapping $\beta \in \mathcal{O}_{\text {exp }}^{\star}(H) \mapsto\left(\delta^{s} \circ \varphi\right)(\beta)$ is a continuous functional on the space $\mathcal{O}_{\text {exp }}^{\star}(H)$, i.e. by stereotype duality, an element of the space $\mathcal{O}_{\exp }(H)$ :

$$
\left(\delta^{s} \circ \varphi\right)(\beta)=\beta(v), \quad v \in \mathcal{O}_{\exp }(H)
$$

Thus

$$
w(s, t)=\varphi\left(\delta^{t}\right)(s)=\left(\delta^{s} \circ \varphi\right)\left(\delta^{t}\right)=\delta^{t}(v)=v(t)
$$

i.e. the function $w(s, \cdot)$ is holomorphic with respect to the second variable.

Further, turning the chain of item 6 to reverse direction, we obtain:

$$
\begin{aligned}
& \varphi \in g \text { \ } h^{\mathbf{I}} \\
& \Downarrow \\
& \varphi\left(\left(h^{\mathbf{V}}\right)^{\circ}\right) \subseteq g^{\mathbf{\bullet}} \\
& \Downarrow \\
& \forall \beta \in(h)^{\circ} \quad \varphi(\beta) \in g^{\mathbf{\square}} \\
& \Downarrow \\
& \forall t \in H \quad \frac{1}{h(t)} \delta^{t} \in(h)^{\circ} \quad \Longrightarrow \quad \varphi\left(\frac{1}{h(t)} \delta^{t}\right) \in g \\
& \Downarrow \\
& \forall t \in H \quad \varphi\left(\delta^{t}\right) \in h(t) \cdot g \\
& \Downarrow \\
& \forall s \in G \quad \forall t \in H \quad|w(s, t)|=\left|\varphi\left(\delta^{t}\right)(s)\right| \leqslant h(t) \cdot g(s)=|(g \boxminus h)(s, t)| \\
& \Downarrow \\
& w \in(g \boxminus h)
\end{aligned}
$$

And finally it remains to note that $w$ is an inverse image of $\varphi$ under the mapping $w \mapsto \rho_{G, H}(w)$ :

$$
\begin{gathered}
\forall s, t \quad \rho_{G, H}(w)\left(\delta^{t}\right)(s)=\left(\delta^{t} \circ \widehat{w}\right)(s)=\delta^{t}(\widehat{w}(s))=\widehat{w}(s)(t)=w(s, t)=\varphi\left(\delta^{t}\right)(s) \\
\Downarrow \\
\rho_{G, H}(w)=\varphi
\end{gathered}
$$

9. Thus, we obtained that the mapping

$$
w \in(g \sqcup h) \quad \mapsto \quad \rho_{G, H}(w) \in g \text { 】 } \odot h^{\mathbf{V}}
$$

is bijective. It remains to show now that it is continuous in both directions. This follows from the fact that both $(g \boxminus h)$ and $g \bullet h$ are compact sets. Since functionals $\delta^{s} \circledast \delta^{t}$ separate the points of the compact set $g \odot h$, the (Hausdorff) topology they generate on $g \odot h^{\mathbf{\bullet}}$ coincides with the initial topology of $g \boldsymbol{L}^{\mathbf{\bullet}}:$ if

$$
\delta^{s} \circledast \delta^{t}\left(\varphi_{i}\right) \underset{i \rightarrow \infty}{\longrightarrow} \delta^{s} \circledast \delta^{t}(\varphi)
$$

for any $s \in G, t \in H$, then

$$
\varphi_{i} \underset{i \rightarrow \infty}{\stackrel{g_{\odot}}{\longrightarrow} \varphi} \varphi
$$

From this we have that the mapping $w \mapsto \rho_{G, H}(w)$ is continuous in forward direction:

$$
w_{i} \underset{i \rightarrow \infty}{\stackrel{(g \boxminus h)}{\longrightarrow}} w
$$




$$
\begin{aligned}
& \Downarrow \\
& \forall(s, t) \in G \times H \quad \delta^{s} \circledast \delta^{t}\left(\rho_{G, H}\left(w_{i}\right)\right)=w_{i}(s, t) \underset{i \rightarrow \infty}{\longrightarrow} w(s, t)=\delta^{s} \circledast \delta^{t}\left(\rho_{G, H}(w)\right) \\
& \Downarrow \\
& \rho_{G, H}\left(w_{i}\right) \underset{i \rightarrow \infty}{\stackrel{g}{\mathbf{I}_{\odot} \mathbf{|}}} \rho_{G, H}(w)
\end{aligned}
$$

Thus, the operation $w \mapsto \rho_{G, H}(w)$ is a continuous bijective mapping of the compact set $(g \square h)$ into the compact set $g \bullet h$. This means that $\rho_{G, H}$ is a homeomorphism between $(g \sqcup h)$ and $g \bullet h$.

Proof of Theorem 4.11. Let us note from the very beginning that if $f$ is a semicharacter on $G \times H$, then the functions

$$
g(s)=f\left(s, 1_{H}\right), \quad h(t)=f\left(1_{G}, t\right), \quad s \in G, t \in H
$$

are again semicharacters (as restrictions of $f$ on subgroups), and the function $g \square h$ is a semicharacter on $G \times H$, majorizing $f$ :

$$
f \leqslant g \boxminus h
$$

Indeed,

$$
f(s, t)=f\left(\left(s, 1_{H}\right) \cdot\left(1_{G}, t\right)\right) \leqslant f\left(s, 1_{H}\right) \cdot f\left(1_{G}, t\right)=g(s) \cdot h(t)=(g \square h)(s, t)
$$

From this it follows that every function $w \in \mathcal{O}_{\exp }(G \times H)$ is contained in some compact set of the form $(g \sqcup h)$ (since $w$ is always contained in compact set of the form $f \mathbf{U}$ ). At the same time the object $\rho_{G, H}(w)$ is an element of the set $g \boldsymbol{\Downarrow} \odot h^{\mathbf{\bullet}}$, i.e. an element of the space $\mathcal{O}_{\exp }(G) \oslash \mathcal{O}_{\text {exp }}^{\star}(H)$.

Thus, Formulas (4.42) and (4.41) correctly define a mapping

$$
w \in \mathcal{O}_{\exp }(G \times H) \quad \mapsto \quad \rho_{G, H}(w) \in \mathcal{O}_{\exp }(G) \oslash \mathcal{O}_{\text {exp }}^{\star}(H)
$$

and we only need to check its bijectivity and continuity in both directions.

1. The injectivity of $\rho_{G, H}$ follows from its injectivity on compact sets $(g \square h)$ (and from the fact that the compact sets $(g \sqcup h)$ and $g \bullet h$ are injectively included into the spaces $\mathcal{O}_{\exp }(G \times H)$ and $\left.\mathcal{O}_{\exp }(G) \oslash \mathcal{O}_{\text {exp }}^{\star}(H)\right)$.

2. The surjectivity of $\rho_{G, H}$ follows from the fact that it surjectively maps compact sets $(g \sqcup h)$ into compact sets $g-{ }^{\top}$ (and from the fact that the compact sets $g \odot h$ cover all the space $\left.\mathcal{O}_{\exp }(G) \oslash \mathcal{O}_{\text {exp }}^{\star}(H)\right)$.

3. The continuity follows from the fact that $\rho_{G, H}$ continuously maps every compact set $(g \square h)$ into the compact set $g \bullet h$, hence into the space $\mathcal{O}_{\exp }(G) \oslash \mathcal{O}_{\text {exp }}^{\star}(H)$. This means that $\rho_{G, H}$ is continuous on each compact set $K$ in the Brauner space $\mathcal{O}_{\exp }(G \times H)$, thus it must be continuous on the whole space $\mathcal{O}_{\exp }(G \times H)$.

4. The continuity in reverse direction is proved in the same way: since the inverse mapping continuously turns every compact set $g$ \ $h^{\mathbf{}}$ into a compact set $(g \boxminus h)$, it is continuous on each compact set in the Brauner space $\mathcal{O}_{\exp }(G) \oslash \mathcal{O}_{\text {exp }}^{\star}(H)$. Thus, it is continuous on the whole space $\mathcal{O}_{\exp }(G) \oslash \mathcal{O}_{\text {exp }}^{\star}(H)$.

5. We have proved that the mapping defined by formulas (4.41) - (4.42) is an isomorphism of the stereotype spaces: $\mathcal{O}_{\exp }(G \times H) \cong \mathcal{O}_{\exp }(G) \oslash \mathcal{O}_{\text {exp }}^{\star}(H)=\mathcal{O}_{\exp }(G) \odot \mathcal{O}_{\exp }(H)$ (hence the identities (4.43) hold). Let us show that this mapping satisfies the identity (4.40): if $u \in \mathcal{O}_{\exp }(G), v \in \mathcal{O}_{\exp }(H)$, then for the mapping $\widehat{u \boxminus v}: G \rightarrow \mathcal{O}_{\exp }(H)$ defined by formula (4.42) we have the following logic chain:

$$
\begin{gathered}
\widehat{u \boxminus v}(s)(t)=(u \boxminus v)(s, t)=u(s) \cdot v(t) \\
\Downarrow \\
\widehat{u \boxminus v}(s)=u(s) \cdot v \\
\Downarrow \\
\forall \beta \in \mathcal{O}_{\text {exp }}^{\star}(H) \quad \beta(\widehat{u \boxminus v}(s))=u(s) \cdot \beta(v)
\end{gathered}
$$




$$
\begin{gathered}
\Downarrow \\
\forall \beta \in \mathcal{O}_{\exp }^{\star}(H) \quad \rho_{G, H}(u \boxminus v)(\beta)=\beta \circ \widehat{u \boxminus v}=\beta(v) \cdot u=(\underline{1.2})=(u \odot v)(\beta) \\
\Downarrow \\
\rho_{G, H}(u \boxminus v)=u \odot v
\end{gathered}
$$

It remains now to note that since the elements of the form $u \circledast v$ generate a dense subspace in $\mathcal{O}_{\exp }(G) \circledast$ $\mathcal{O}_{\exp }(H)$, by the proven above nuclearity of the spaces $\mathcal{O}_{\exp }$, the corresponding elements of the form $u \odot v$ must generate a dense subspace in $\mathcal{O}_{\exp }(G) \odot \mathcal{O}_{\exp }(H)$, and elements $u \boxminus v$ a dense subspace in $\mathcal{O}_{\exp }(G \times H)$. This implies that the property (4.40) uniquely define the mapping $\rho_{G, H}$.

\section{(h) Structure of Hopf algebras on $\mathcal{O}_{\exp }(G)$ and $\mathcal{O}_{\text {exp }}^{\star}(G)$}

In $\S 3(\mathrm{~b})$ we have mentioned the standard trick, allowing to prove that functional algebras of a given class on groups are Hopf algebras - a sufficient condition for this is a natural isomorphism between the functional algebra of the Cartesian product of groups $\times$ and the corresponding tensor product of their functional algebras. Theorem 4.11 establishing the natural isomorphism

$$
\mathcal{O}_{\exp }(G \times H) \stackrel{\rho_{G, H}}{\cong} \mathcal{O}_{\exp }(G) \odot \mathcal{O}_{\exp }(H)
$$

allows now to make the same conclusion about algebras $\mathcal{O}_{\exp }(G)$ :

Theorem 4.12. For any compactly generated Stein group $G$

- the space $\mathcal{O}_{\exp }(G)$ of holomorphic functions of exponential type on $G$ is a nuclear Hopf-Brauner algebra with respect to the algebraic operations defined by formulas, analogous to (3.3) -(3.7);

- its stereotype dual space $\mathcal{O}_{\exp }^{\star}(G)$ is a nuclear Hopf-Fréchet algebra with respect to dual algebraic operations.

\section{$\S 5$ Arens-Michael envelope and holomorphic reflexivity}

\section{(a) Submultiplicative seminorms and Arens-Michael algebras}

A seminorm $p: A \rightarrow \mathbb{R}_{+}$on an algebra $A$ is said to be submultiplicative, if it satisfies the following condition

$$
p(u \cdot v) \leqslant p(u) \cdot p(v), \quad u, v \in A
$$

This is equivalent to the fact that the unit ball of this seminorm

$$
U=\{u \in A: p(u) \leqslant 1\}
$$

satisfies the condition

$$
U \cdot U \subseteq U
$$

(such sets in $A$ are also said to be submultiplicative like submultiplicative neighborhoods of zero, defined on page 66).

A topological algebra $A$ is called an Arens-Michael algebra, if it is complete (as a topological vector space) and satisfies the following equivalent conditions:

(i) the topology of $A$ is generated by a system of submultiplicative seminorms:

(ii) $A$ has a local base of submultiplicative closed absolutely convex neighborhoods of zero. 
Example 5.1. Seminorms (3.20) generating the topology on $\mathcal{O}(\mathbb{Z})$ are submultiplicative:

$$
\|u\|_{N}=\sum_{|n| \leqslant N}|u(n)|, \quad N \in \mathbb{N},
$$

hence $\mathcal{O}(\mathbb{Z})$ is an Arens-Michael algebra.

Proof. Indeed,

$\|u \cdot v\|_{N}=\sum_{|n| \leqslant N}|(u \cdot v)(n)|=\underline{(3.19)}=\sum_{|n| \leqslant N}|u(n) \cdot v(n)| \leqslant\left(\sum_{|n| \leqslant N}|u(n)|\right) \cdot\left(\sum_{|n| \leqslant N}|v(n)|\right)=\|u\|_{N} \cdot\|v\|_{N}$

Example 5.2. Seminorms (3.47) generating the topology on $\mathcal{O}\left(\mathbb{C}^{\times}\right)$, are submultiplicative:

$$
\|u\|_{C}=\sum_{n \in \mathbb{Z}}\left|u_{n}\right| \cdot C^{|n|}, \quad C \geqslant 1,
$$

hence $\mathcal{O}\left(\mathbb{C}^{\times}\right)$is an Arens-Michael algebra.

Proof. Indeed,

$$
\begin{aligned}
& \|u \cdot v\|_{C}=\sum_{n \in \mathbb{Z}}\left|(u \cdot v)_{n}\right| \cdot C^{|n|}=(\underline{3.46})=\sum_{n \in \mathbb{Z}}\left|\sum_{i \in \mathbb{Z}} u_{i} \cdot v_{n-i}\right| \cdot C^{|n|} \leqslant \\
& \quad \leqslant \sum_{n \in \mathbb{Z}} \sum_{i \in \mathbb{Z}}\left|u_{i}\right| \cdot\left|v_{n-i}\right| \cdot C^{|i|} \cdot C^{|n-i|}=\left(\sum_{k \in \mathbb{Z}}\left|u_{k}\right| \cdot C^{|k|}\right) \cdot\left(\sum_{l \in \mathbb{Z}}\left|u_{l}\right| \cdot C^{|l|}\right)=\|u\|_{C} \cdot\|v\|_{C}
\end{aligned}
$$

Example 5.3. Seminorms (3.72), generating the topology on $\mathcal{O}(\mathbb{C})$, are submultiplicative:

$$
\|u\|_{C}=\sum_{n=0}^{\infty}\left|u_{n}\right| \cdot C^{n}, \quad C \geqslant 0,
$$

hence $\mathcal{O}(\mathbb{C})$ is an Arens-Michael algebra.

Proof. Indeed,

$$
\begin{array}{r}
\|u \cdot v\|_{C}=\sum_{n=0}^{\infty}\left|(u \cdot v)_{n}\right| \cdot C^{n}=(\underline{3.71})=\sum_{n=0}^{\infty}\left|\sum_{i=0}^{n} u_{i} \cdot v_{n-i}\right| \cdot C^{n} \leqslant \sum_{n=0}^{\infty} \sum_{i=0}^{n}\left|u_{i}\right| \cdot\left|v_{n-i}\right| \cdot C^{n}= \\
=\left(\sum_{k \in \mathbb{N}}\left|u_{k}\right| \cdot C^{k}\right) \cdot\left(\sum_{l=0}^{\infty}\left|u_{l}\right| \cdot C^{l}\right)=\|u\|_{C} \cdot\|v\|_{C}
\end{array}
$$

Of the following three propositions the first two are evident, and the third one follows from the A. Yu. Pirkovskii theorem [5.1] which we formulate below in this section:

Proposition 5.1. The algebra $\mathcal{O}(M)$ of holomorphic functions on any complex manifold $M$ is an ArensMichael algebra.

Proposition 5.2. The algebra $\mathcal{O}_{\text {exp }}^{\star}(G)$ of exponential functionals on every Stein group is an ArensMichael algebra.

Proposition 5.3. The algebra $\mathcal{R}(M)$ of polynomials on a complex affine algebraic manifold $M$, endowed with the strongest locally convex topology, is an Arens-Michael algebra if an only if the manifold $M$ is finite. 


\section{(b) Arens-Michael envelopes}

The Arens-Michael envelope of a topological algebra $A$ is a (continuous) homomorphism $\pi: A \rightarrow B$ of $A$ into an Arens-Michael algebra $B$ such that for any (continuous) homomorphism $\rho: A \rightarrow C$ of $A$ into an arbitrary Arens-Michael algebra $C$ there is a unique (continuous) homomorphism $\sigma: B \rightarrow C$ such that the following diagram is commutative:

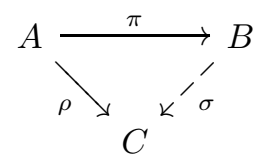

From this definition it is clear that if $\pi: A \rightarrow B$ and $\rho: A \rightarrow C$ are two Arens-Michael envelopes of $A$, then the arising homomorphism $\sigma: B \rightarrow C$ becomes an isomorphism of topological algebras (due to the uniqueness of $\sigma$ ). Hence the Arens-Michael envelope of $A$ is defined uniquely up to an isomorphism, and as a corollary, we can introduce a special notation for this construction:

$$
\varnothing_{A}: A \rightarrow A^{\odot}
$$

This should be understood as follows: if we have a homomorphism $\varphi: A \rightarrow B$, then the record $\varphi=\varnothing_{A}$ means that $\varphi: A \rightarrow B$ is an Arens-Michael envelope of the algebra $A$; on the other hand, if we have an algebra $B$, then the record $B=A^{\odot}$ means that there exists a homomorphism $\varphi: A \rightarrow B$ which is an Arens-Michael envelope of the algebra $A$ - in this case the algebra $B$ is also called an Arens-Michael envelope of the algebra $A$.

Proposition 5.4. A topological algebra $B$ is an Arens-Michael envelope for a topological algebra $A$ if and only if

(i) $B$ is an Arens-Michael algebra, and

(ii) there exists a continuous homomorphism $\pi: A \rightarrow B$ such that

(a) the image $\pi(A)$ of the algebra $A$ under the action of $\pi$ is dense in $B$,

(b) for any continuous submultiplicative seminorm $p: A \rightarrow \mathbb{R}_{+}$there is a continuous submultiplicative seminorm $\widetilde{p}: B \rightarrow \mathbb{R}_{+}$, such that the seminorm $\widetilde{p} \circ \pi: A \rightarrow \mathbb{R}_{+}$majorizes the seminorm $p$ :

$$
p(a) \leqslant \widetilde{p}(\pi(a)), \quad a \in A
$$

The Arens-Michael envelope can be constructed directly: to any submultiplicative neighborhood of zero $U$ in $A$ we can assign the closed ideal $\operatorname{Ker} U$ in $A$ defined by the equality

$$
\operatorname{Ker} U=\bigcap_{\varepsilon>0} \varepsilon \cdot U
$$

and a quotient algebra

$$
A / \operatorname{Ker} U
$$

endowed with the topology of normed space with the unit ball $U+\operatorname{Ker} U$. Then the completion $(A / \operatorname{Ker} U)^{\mathbf{}}$ becomes a Banach algebra. Following (0.3) we denote such algebra by $A / U$ :

$$
A / U:=(A / \operatorname{Ker} U)^{\boldsymbol{\nabla}}
$$

The family of such algebras (with different $U$ ) forms a projective system. The Its limit is an Arens-Michael envelope $A^{\odot}$ :

$$
\begin{aligned}
& A^{\odot}=\lim _{\longleftarrow} A / U . \\
& U \text { is a submultiplicative } \\
& \text { neighborhood of zero in } A
\end{aligned}
$$

The following propositions show that the operation of taking the Arens-Michael envelope commutes with the passage to direct sums and quotient algebras. 
Proposition 5.5. The Arens-Michael envelope of a direct sum $A_{1} \oplus \ldots \oplus A_{n}$ of finite family of topological algebras $A_{1}, \ldots, A_{n}$ coincides with the direct sum of the Arens-Michael envelopes of these algebras:

$$
\left(A_{1} \oplus \ldots \oplus A_{n}\right)^{\odot} \cong A_{1}^{\odot} \oplus \ldots \oplus A_{n}^{\odot}
$$

Proposition 5.6. Let $\pi: A \rightarrow A^{\diamond}$ be an Arens-Michael envelope of the algebra A, and let I be a close $d$ ideal in $A$. Then the Arens-Michael envelope of the quotient algebra $A / I$ coincides with the completion of the quotient algebra $A^{\odot} \overline{\pi(I)}$ over the closure $\overline{\pi(I)}$ in $A^{\curvearrowright}$ of the image of ideal I under the action of the mapping $\pi$ :

$$
(A / I)^{\odot} \cong\left(A^{\odot} \overline{\pi(I)}\right)^{\boldsymbol{\nabla}}
$$

An important example of the Arens-Michael envelope was constructed by A .Yu. Pirkovskii:

Theorem 5.1 (A .Yu. Pirkovskii, 26]). The Arens-Michael envelope of the algebra $\mathcal{R}(M)$ of polynomials on an affine algebraic manifold $M$ coincides with the algebra $\mathcal{O}(M)$ of holomorphic functions on $M$ :

$$
(\mathcal{R}(M))^{\odot} \cong \mathcal{O}(M)
$$

(c) The mapping $b_{G}^{\star}: \mathcal{O}^{\star}(G) \rightarrow \mathcal{O}_{\exp }^{\star}(G)$ is an Arens-Michael envelope

Theorem 5.2. For any Stein group $G$ the mapping

$$
b_{G}^{\star}: \mathcal{O}^{\star}(G) \rightarrow \mathcal{O}_{\text {exp }}^{\star}(G)
$$

is an Arens-Michael envelope of the algebra $\mathcal{O}^{\star}(G)$ :

$$
\left(\mathcal{O}^{\star}(G)\right)^{\odot} \cong \mathcal{O}_{\text {exp }}^{\star}(G)
$$

Proof. From the representation (4.17) it follows that this space is a projective limit of the Banach quotient algebras:

$$
\begin{aligned}
& \mathcal{O}_{\text {exp }}^{\star}(G)=(4.17)=\left(\underset{\begin{array}{c}
D \text { is a dually } \\
\text { submultiplicative } \\
\text { rectangle in } \mathcal{O}(G)
\end{array}}{\lim } \mathbb{C} D\right)^{\star}=\underset{\begin{array}{c}
D \text { is a dually } \\
\text { submultiplicative } \\
\text { rectangle in } \mathcal{O}(G)
\end{array}}{\lim (\mathbb{C} D)^{\star}}=\underbrace{(0.4)}_{\begin{array}{c}
D \text { is a dually } \\
\text { submultiplicative } \\
\text { rectangle in } \mathcal{O}(G)
\end{array}}=\lim ^{\star}(G) / D^{\circ}= \\
& =\lim _{\Delta \text { is a submultiplicative }} \mathcal{O}^{\star}(G) / \Delta=(\text { Theorem } 4.2(\mathrm{a}))=\lim _{U \text { is a submultiplicative }} \mathcal{O}^{\star}(G) / U=(\underline{5.1})=\left(\mathcal{O}^{\star}(G)\right)^{\odot} \\
& \text { rhombus in } \mathcal{O}^{\star}(G) \\
& \begin{array}{c}
U \text { is a submultiplicative } \\
\text { neighborhood of zero in } \mathcal{O}^{\star}(G)
\end{array}
\end{aligned}
$$

(d) The mapping $b_{G}: \mathcal{O}_{\exp }(G) \rightarrow \mathcal{O}(G)$ is an Arens-Michael envelope for the groups with algebraic connected component of identity

Theorem 5.3. Let $G$ be a compactly generated Stein group, such that the connected component of identity $G_{e}$ is an algebraic group. Then the mapping

$$
b_{G}: \mathcal{O}_{\exp }(G) \rightarrow \mathcal{O}(G)
$$

is an Arens-Michael envelope of the algebra $\mathcal{O}_{\exp }(G)$ :

$$
\left(\mathcal{O}_{\exp }(G)\right)^{\odot} \cong \mathcal{O}(G)
$$


We shall prove this theorem in several steps.

1. Let initially $G$ be a discrete group. Recall that in (0.28) we agreed to denote by $1_{x}$ the characteristic functions of singletons $\{x\}$ in $G$ :

$$
1_{x}(y)= \begin{cases}1, & y=x \\ 0 & y \neq x\end{cases}
$$

(since $G$ is discrete, the function $1_{x}$ can be considered as element of both algebras $\mathcal{O}(G)$ and $\mathcal{O}_{\exp }(G)$ ).

Lemma 5.1. The functions $\left\{1_{x} ; x \in G\right\}$ form a basis in the topological vector spaces $\mathcal{O}(G)$ and $\mathcal{O}_{\exp }(G)$ : for any function $u \in \mathcal{O}(G)\left(u \in \mathcal{O}_{\exp }(G)\right)$ the following equality holds

$$
u=\sum_{x \in G} u(x) \cdot 1_{x}
$$

where the series converges in $\mathcal{O}(G)\left(\mathcal{O}_{\exp }(G)\right)$, and its coefficients continuously depend on $u \in \mathcal{O}(G)$ $\left(u \in \mathcal{O}_{\exp }(G)\right)$.

Proof. For the space $\mathcal{O}(G)$ this is evident, since for the case of discrete group $G$ this space coincides with the space $\mathbb{C}^{G}$ of all functions on $G$. Let us prove this for $\mathcal{O}_{\exp }(G)$ : if $u \in \mathcal{O}_{\exp }(G)$, then taking a majorizing semicharacter $f: G \rightarrow \mathbb{R}_{+}$,

$$
|u(x)| \leqslant f(x), \quad x \in G
$$

we obtain that the partial sums of the series (5.6) are contained in the rectangle $f \mathbf{\bullet}$, so the series (5.6) converges (not only in $\mathcal{O}(G)$, but) in $\mathcal{O}_{\exp }(G)$ as well. On the other hand, every coefficient $u(x)$ continuously depend $u$, if $u$ runs over the rectangle $f \bullet$. By the definition of topology in $\mathcal{O}_{\exp }(G)$, this means that $u(x)$ continuously depend on $u$, when $u$ runs over $\mathcal{O}_{\exp }(G)$.

Lemma 5.2. If $G$ is a discrete finitely generated group, then for any continuous seminorm $q: \mathcal{O}_{\exp }(G) \rightarrow$ $\mathbb{R}_{+}$and for any semicharacter $f: G \rightarrow[1 ;+\infty)$ the number family $\left\{f(x) \cdot q\left(1_{x}\right) ; x \in G\right\}$ is summable:

$$
\sum_{x \in G} f(x) \cdot q\left(1_{x}\right)<\infty
$$

Proof. Let $T$ is an absolutely convex compact set in $\mathcal{O}_{\text {exp }}^{\star}(G)$ corresponding to the seminorm $q$ :

$$
q(u)=\sup _{\alpha \in T}|\alpha(u)|
$$

Every rectangle $f$ is a compact set in $\mathcal{O}_{\text {exp }}^{\star}(G)$, so

$$
\begin{array}{r}
\infty>\sup _{u \in f} \sup _{\alpha \in T}|\alpha(u)|=(\underline{5.6})=\sup _{u \in f} \sup _{\alpha \in T}\left|\alpha\left(\sum_{x \in G} u(x) \cdot 1_{x}\right)\right|=\sup _{u \in f} \sup _{\alpha \in T}\left|\sum_{x \in G} u(x) \cdot \alpha\left(1_{x}\right)\right| \geqslant \\
\geqslant \sup _{\alpha \in T}|\sum_{x \in G} \underbrace{f(x) \cdot \overline{\alpha\left(1_{x}\right)}}_{\begin{array}{c}
\uparrow \\
\text { one of the values of } \\
u \in f
\end{array}} \cdot \alpha\left(1_{x}\right)|=\sup _{\alpha \in T} \sum_{x \in G} f(x) \cdot\left|\alpha\left(1_{x}\right)\right| \geqslant \sup _{\alpha \in T} \sup _{x \in G} f(x) \cdot\left|\alpha\left(1_{x}\right)\right|= \\
=\sup _{x \in G} f(x) \cdot \sup _{\alpha \in T}\left|\alpha\left(1_{x}\right)\right|=\sup _{x \in G} f(x) \cdot q\left(1_{x}\right)
\end{array}
$$

So we have that for any semicharacter $f: G \rightarrow[1 ;+\infty)$

$$
\sup _{x \in G} f(x) \cdot q\left(1_{x}\right)<\infty
$$


Now take a finite set $K$, generating $G$,

$$
\bigcup_{n=1}^{\infty} K^{n}=G
$$

and define a semicharacter $g: G \rightarrow[1 ;+\infty)$ by formula

$$
g(x)=R^{n} \quad \Longleftrightarrow \quad x \in K^{n} \backslash K^{n-1}
$$

where $R$ is a number, greater than the cardinality of $K$ :

$$
R>\operatorname{card} K \text {. }
$$

Since the product $g \cdot f$ is also a semicharacter, we have:

$$
\begin{gathered}
\sup _{x \in G}\left[g(x) \cdot f(x) \cdot q\left(1_{x}\right)\right]<\infty \\
\Downarrow \\
\exists C>0 \quad \forall x \in G \quad f(x) \cdot q\left(1_{x}\right) \leqslant \frac{C}{g(x)} \\
\Downarrow \\
\sum_{x \in G} f(x) \cdot q\left(1_{x}\right) \leqslant \sum_{x \in G} \frac{C}{g(x)}=\sum_{n=1}^{\infty} \sum_{x \in K^{n} \backslash K^{n-1}} \frac{C}{g(x)}=\sum_{n=1}^{\infty} \sum_{x \in K^{n} \backslash K^{n-1}} \frac{C}{R^{n}} \leqslant \sum_{n=1}^{\infty} \frac{C \cdot \operatorname{card}\left(K^{n}\right)}{R^{n}} \leqslant \\
\end{gathered}
$$

If $q: \mathcal{O}_{\exp }(G) \rightarrow \mathbb{R}_{+}$is a continuous seminorm on $\mathcal{O}_{\exp }(G)$, then let us call its support the set

$$
\operatorname{supp}(q)=\left\{x \in G: q\left(1_{x}\right) \neq 0\right\}
$$

Lemma 5.3. If $G$ is a discrete finitely generated group, then for any submultiplicative continuous seminorm $q: \mathcal{O}_{\exp }(G) \rightarrow \mathbb{R}_{+}$

(a) its support $\operatorname{supp}(q)$ is a finite set:

$$
\operatorname{card} \operatorname{supp}(q)<\infty
$$

(b) for any point $x \in \operatorname{supp}(q)$ the value of the seminorm $q$ on any function $1_{x}$ is less than 1:

$$
q\left(1_{x}\right) \geqslant 1
$$

Proof. Let us prove (b) first. If $x \in \operatorname{supp}(q)$, i.e. $q\left(1_{x}\right)>0$, then:

$$
1_{x}=1_{x}^{2} \quad \Longrightarrow \quad q\left(1_{x}\right)=q\left(1_{x}^{2}\right) \leqslant q\left(1_{x}\right)^{2} \quad \Longrightarrow \quad 1 \leqslant q\left(1_{x}\right)
$$

Now (a). Since the constant identity $f(x)=1$ is a semicharacter on $G$, by Lemma 5.2 the number family $\left\{q\left(1_{x}\right) ; x \in G\right\}$ is summing:

$$
\sum_{x \in G} q\left(1_{x}\right)<\infty
$$

On the other hand, by the condition (b) we have already proved, all non-zero terms in this series are bounded from below by 1 . Hence there is a finite number of them. 
2. Let us now pass to the case when $G$ is compactly generated Stein group, whose connected component of identity $G_{e}$ is an algebraic group. Let $\mathcal{L} C_{\text {exp }}(G)$ denote a subalgebra in $\mathcal{O}_{\text {exp }}(G)$ consisting of locally constant functions:

$$
u \in \mathcal{L} C_{\exp }(G) \quad \Longleftrightarrow \quad u \in \mathcal{O}_{\exp }(G) \quad \& \quad(\forall x \in G \quad \exists \operatorname{neighborhood} U \ni x \quad \forall y \in U \quad u(x)=u(y))
$$

Lemma 5.4. Let $\pi: G \rightarrow G / G_{e}$ denote the quotient mapping. For any function $v \in \mathcal{O}_{\exp }\left(G / G_{e}\right)$ the composition $v \circ \pi$ is a locally constant function of exponential type on $G$, and the mapping

$$
v \mapsto v \circ \pi
$$

establishes an isomorphism of topological algebras:

$$
\mathcal{O}_{\exp }\left(G / G_{e}\right) \cong \mathcal{L} C_{\exp }(G)
$$

Let now for any coset $K \in G / G_{e}$ and for any function $u \in \mathcal{O}_{\exp }(G)$ the symbol $u_{K}$ denote the function coinciding with $u$ on the set $K \subset G$ and vanishing outside of $K$ :

$$
u_{K}(x)= \begin{cases}u(x), & x \in K \\ 0, & x \notin K\end{cases}
$$

The following proposition is proved just like Lemma 5.1

Lemma 5.5. For any coset $K \in G / G_{e}$ and for any function $u \in \mathcal{O}_{\exp }(G)$

(a) the function $u_{K}$ belongs to algebra $\mathcal{O}_{\exp }(G)$,

(b) the mapping $u \in \mathcal{O}_{\exp }(G) \mapsto u_{K} \in \mathcal{O}_{\exp }(G)$ is continuous, and

(c) the series $\sum_{K \in G / G_{e}} u_{K}$ converges in the space $\mathcal{O}_{\exp }(G)$ to the function $u$ :

$$
u=\sum_{K \in G / G_{e}} u_{K}
$$

For any coset $K \in G / G_{e}$ let us consider the operator of projection

$$
P_{K}: \mathcal{O}_{\exp }(G) \rightarrow \mathcal{O}_{\exp }(G), \quad P_{K}(u)=u_{K}
$$

and let $\mathcal{O}_{\exp }(K)$ denote its image in the space $\mathcal{O}_{\exp }(G)$ :

$$
\mathcal{O}_{\exp }(K)=P_{K}\left(\mathcal{O}_{\exp }(G)\right)
$$

Clearly, $\mathcal{O}_{\exp }(K)$ is a closed subspace in $\mathcal{O}_{\exp }(G)$, so $\mathcal{O}_{\exp }(K)$ can be endowed with the topology induced from $\mathcal{O}_{\exp }(G)$ (this will be the same as the topology of an immediate subspace in $\mathcal{O}_{\exp }(G)$ ), and with respect to this topology $\mathcal{O}_{\exp }(K)$ is a Brauner space.

Let in addition $\mathcal{O}(K)$ denote the usual algebra of holomorphic functions on the complex manifold $K$.

Lemma 5.6. The inclusion $\mathcal{O}_{\exp }(K) \subseteq \mathcal{O}(K)$ is an Arens-Michael envelope:

$$
\mathcal{O}_{\exp }(K)^{\ominus}=\mathcal{O}(K)
$$

Proof. We have to note first that it is sufficient to consider the case of $K=G_{e}$, since shifts turn inclusions $\mathcal{O}_{\exp }(K) \subseteq \mathcal{O}(K)$ into inclusions $\mathcal{O}_{\exp }\left(G_{e}\right) \subseteq \mathcal{O}\left(G_{e}\right)$. For this case our proposition becomes a corollary of the Pirkovskii Theorem 5.1 by assumption, $G_{e}$ is an algebraic group, so we can consider the algebra 
$\mathcal{R}\left(G_{e}\right)$ of polynomials on $G_{e}$. Then the train of thought is illustrated by the following diagram (where the horizontal arrows mean inclusions):

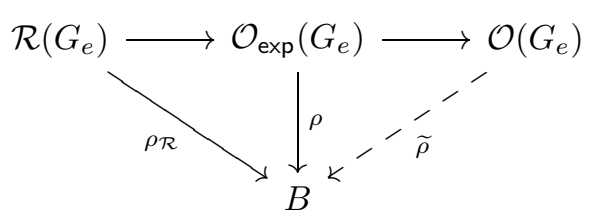

If $\rho: \mathcal{O}_{\exp }\left(G_{e}\right) \rightarrow B$ is an arbitrary morphism into the Arens-Michael algebra in $B$, then by Pirkovskii's Theorem there arises a unique morphism $\rho_{\mathcal{R}}: \mathcal{R}\left(G_{e}\right) \rightarrow B$. Since $\mathcal{R}\left(G_{e}\right)$ is dense in $\mathcal{O}_{\exp }\left(G_{e}\right)$ in the topology of $\mathcal{O}\left(G_{e}\right)$, the morphism $\widetilde{\rho}$ extends the morphism $\rho$. And since $\mathcal{O}_{\exp }\left(G_{e}\right)$ is dense in $\mathcal{O}\left(G_{e}\right)$, this extension is unique.

Proof of Theorem 5.3. Let $G$ be an arbitrary compactly generated Stein group with algebraic connected component of identity and $p: \mathcal{O}_{\exp }(G) \rightarrow \mathbb{R}_{+}$a submultiplicative seminorm. Its restriction $\left.p\right|_{\mathcal{L} C_{\text {exp }}(G)}$ to the subalgebra $\mathcal{L} C_{\text {exp }}(G)$ defines by Lemma 5.4 a continuous seminorm $q$ on $\mathcal{O}_{\exp }\left(G / G_{e}\right)$,

$$
q(v)=p(v \circ \pi)
$$

and $q$ will be submultiplicative, like $p$. Hence by Lemma 5.3, the support of $q$ is finite:

$$
\operatorname{card} \operatorname{supp}(q)<\infty
$$

Being applied to seminorm $p$ this mean that there exists a finite family of cosets $\left\{K_{1}, \ldots, K_{n}\right\} \subseteq G / G_{e}$, for which

$$
p\left(1_{K_{i}}\right) \neq 0
$$

while for the others $K \in G / G_{e}, K \notin\left\{K_{1}, \ldots, K_{n}\right\}$,

$$
p\left(1_{K}\right)=0
$$

(here $1_{K}$ denotes the image of the constant identity $u(x)=1$ under the projection (5.8)).

As a corollary, for any function $u \in \mathcal{O}_{\exp }(G)$ and for any coset $K \notin\left\{K_{1}, \ldots, K_{n}\right\}$ we have

$$
p\left(u_{K}\right)=0
$$

(since $p\left(u_{K}\right)=p\left(1_{K} \cdot u_{K}\right) \leqslant p\left(1_{K}\right) \cdot p\left(u_{K}\right)=0 \cdot p\left(u_{K}\right)=0$ ).

Denote now by $P$ and $S$ the projections to the spaces consisting of functions vanishing outside and inside $K_{1} \cup \ldots \cup K_{n}$ :

$$
\begin{gathered}
P(u)(x)=\left\{\begin{array}{cc}
u(x), & x \in K_{1} \cup \ldots \cup K_{n} \\
0, & x \notin K_{1} \cup \ldots \cup K_{n}
\end{array}\right\}=u_{K_{1}}+\ldots+u_{K_{n}} \\
S(u)(x)=\left\{\begin{array}{cc}
u(x), & x \notin K_{1} \cup \ldots \cup K_{n} \\
0, & x \in K_{1} \cup \ldots \cup K_{n}
\end{array}\right\}=\sum_{K \notin\left\{K_{1}, \ldots, K_{n}\right\}} u_{K}
\end{gathered}
$$

(the latter series converges in $\mathcal{O}_{\exp }(G)$, by Lemma 5.5). From (5.10) we have

$$
\forall u \in \mathcal{O}_{\exp }(G) \quad p(S(u))=p\left(\sum_{K \notin\left\{K_{1}, \ldots, K_{n}\right\}} u_{K}\right) \leqslant \sum_{K \notin\left\{K_{1}, \ldots, K_{n}\right\}} p\left(u_{K}\right)=(\overline{5.10})=\sum_{K \notin\left\{K_{1}, \ldots, K_{n}\right\}} 0=0,
$$

what implies

$$
p=p \circ P
$$


(on the one hand, $p(u)=p(P(u)+S(u)) \leqslant p(P(u))+p(S(u))=p(P(u))$, and on the other hand, $p(P(u))=p(u-S(u)) \leqslant p(u)+p(S(u))=p(u))$.

Denote by $p_{i}$ the (submultiplicative) seminorms on $\mathcal{O}_{\exp }\left(K_{i}\right)$, induced by $p$,

$$
p_{i}(v)=p(v), \quad v \in \mathcal{O}_{\exp }\left(K_{i}\right)
$$

By Lemma $\left[5.6\right.$ and Proposition 5.4 these seminorms are majorized by some seminorms $\widetilde{p_{i}}$ and $\mathcal{O}\left(K_{i}\right)$ :

$$
p_{i}(v) \leqslant \widetilde{p_{i}}(v), \quad v \in \mathcal{O}_{\exp }\left(K_{i}\right)
$$

From (5.11) we have the following estimation:

$$
p(u)=p\left(\sum_{i=1}^{n} u_{K_{i}}+\sum_{K \notin\left\{K_{1}, \ldots, K_{n}\right\}} u_{K}\right)=(\underline{5.11})=p\left(\sum_{i=1}^{n} u_{K_{i}}\right) \leqslant \sum_{i=1}^{n} p_{i}\left(u_{K_{i}}\right) \leqslant \sum_{i=1}^{n} \widetilde{p}_{i}\left(u_{K_{i}}\right)
$$

Thus, our initial seminorm $p$ on $\mathcal{O}_{\exp }(G)$ is majorized by the seminorm $\sum_{i=1}^{n} \widetilde{p}_{i}$ on $\mathcal{O}(G)$. Again applying Proposition 5.4 we obtain that the inclusion $\mathcal{O}_{\exp }(G) \subseteq \mathcal{O}(G)$ is an Arens-Michael envelope.

\section{(e) Holomorphic reflexivity}

We can now declare the following a result of our considerations. For a compactly generated Stein group $G$ with the algebraic connected component of identity two algebras of those we considered above, namely, $\mathcal{O}^{\star}(G)$ and $\mathcal{O}_{\exp }(G)$, have the following curious property: every such algebra $H$, being a rigid stereotype Hopf algebra, has an Arens-Michael envelope $H^{\odot}$, which also has a structure of rigid stereotype Hopf algebra, and

(i) the natural homomorphism

$$
\curlyvee_{H}: H \rightarrow H^{\odot}
$$

is a homomorphism of rigid Hopf algebras, and

(ii) the dual mapping

$$
\left(\bigcirc_{H}\right)^{\star}:\left(H^{\odot}\right)^{\star} \rightarrow H^{\star}
$$

is an Arens-Michael envelope of the algebra $\left(H^{\complement}\right)^{\star}$ :

$$
\left(\bigcirc_{H}\right)^{\star}=\varnothing_{\left(H^{\odot}\right) \star}
$$

Let us note the following in view of this:

Proposition 5.7. For an arbitrary rigid stereotype Hopf algebra $H$ the structure of rigid Hopf algebra on the Arens-Michael envelope $H^{\odot}$, satisfying conditions (i) and (ii), if exists, is unique.

Proof. Note first that (i) and (ii) immediately imply

(iii) the mappings $\nabla_{H}$ and $\left(\bigcirc_{H}\right)^{\star}$ are bimorphisms of stereotype spaces (i.e. are injective and have dense image in the range).

Indeed, the mappings $\bigcirc_{H}: H \rightarrow H^{\odot}$ and $\left(\bigcirc_{H}\right)^{\star}:\left(H^{\odot}\right)^{\star} \rightarrow H^{\star}$ are epimorphisms (i.e. have dense image), since they are Arens-Michael envelopes. On the other hand they are dual to each other, so they must be monomorphisms (i.e. are injective).

This implies everything. First, the multiplication and the unit on $H^{\odot}$ are defined uniquely by the condition that $\bigcirc_{H}: H \rightarrow H^{\odot}$ is the Arens-Michael envelope of $H$. Consider the dual mapping $\left(\bigcirc_{H}\right)^{\star}$ : $\left(H^{\bigcirc}\right)^{\star} \rightarrow H^{\star}$. Like $\bigcirc_{H}$, this must be a homomorphism of Hopf algebras. Hence, it is a homomorphism of algebras, and at the same time an injective mapping, by virtue of (iii). This means that the multiplication and the unit in $\left(H^{\odot}\right)^{\star}$ are defined uniquely since they are unduced from $H^{\star}$.

Thus, conditions (i) and (ii) impose rigid conditions on multiplication, unit, comultiplication and counit in $H^{\ominus}$, and allow to define no more than one structure of bialgebra on $H^{\odot}$. On the other hand, we know that antipode, if exists is also unique, so the structure of Hopf algebra on $H^{\odot}$ is also unique. 
It is convenient to sketch out conditions (i) and (ii) by the diagram,

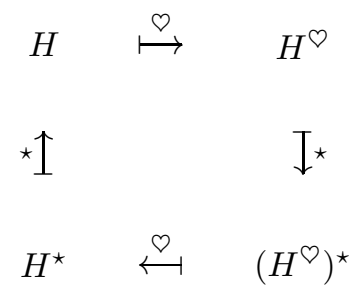

with the following sense: first, in the corners of the square there are rigid stereotype Hopf algebras, and the horizontal arrows (the Arens-Michael operations $\nabla$ ) are their homomorphisms, and, second, the alternation of the operations $\varnothing$ and $\star$ (no matter which place you begin with) at the fourth step returns back to the initial Hopf algebra (of course, up to an isomorphism of functors).

The rigid stereotype Hopf algebras $H$, satisfying conditions (i) and (ii), will be called holomorphically reflexive, and the diagram (5.12) for such algebras the reflexivity diagram. The justification of the term "reflexivity" in this case is the following: if we denote by some symbol, say ${ }^{\wedge}$, the composition of operations $\odot$ and $\star$,

$$
\widehat{H}:=\left(H^{\odot}\right)^{\star}
$$

and call such object a Hopf algebra holomorphically dual to $H$, then $H$ becomes naturally isomorphic to its second dual Hopf algebra:

$$
H \cong \widehat{\widehat{H}}
$$

This is a corollary of Proposition (5.7): since for holomorphically reflexive Hopf algebras the passage $H \mapsto H^{\diamond}$ uniquely defines the structure of Hopf algebra on $H^{\odot}$, the isomorphism of algebras

$$
\left(\left(H^{\odot}\right)^{\star}\right)^{\odot} \cong H^{\star}
$$

postulating in axiom (ii), automatically must be an isomorphism of Hopf algebras. The passage to the dual Hopf algebras exactly gives (5.13).

Theorems 5.2 and 5.3 imply:

Theorem 5.4. If $G$ is a Stein group with the algebraic connected component of identity, then the algebras $\mathcal{O}^{\star}(G)$ and $\mathcal{O}_{\exp }(G)$ are holomorphically reflexive, and the reflexivity diagram for them has the form:

$$
\begin{aligned}
& \mathcal{O}^{\star}(G) \quad \stackrel{\odot}{\stackrel{\odot .3}{(5.3}} \quad \mathcal{O}_{\exp }^{\star}(G) \\
& * \uparrow \quad I * \\
& \mathcal{O}(G) \quad \stackrel{\odot}{\overleftarrow{5.4}} \quad \mathcal{O}_{\exp }(G)
\end{aligned}
$$

(the numbers under the horizontal arrows are references to the formulas in our text).

Example 5.4. For the group $\mathrm{GL}_{n}(\mathbb{C})$ the reflexivity diagram (15.14) is as follows:

$$
\begin{array}{ccc}
\mathcal{O}^{\star}\left(\mathrm{GL}_{n}(\mathbb{C})\right) & \stackrel{\odot}{\stackrel{(4.28}{ }} & \mathcal{R}^{\star}\left(\mathrm{GL}_{n}(\mathbb{C})\right) \\
\star \uparrow & I^{\star} \\
\mathcal{O}\left(\mathrm{GL}_{n}(\mathbb{C})\right) & \stackrel{\odot}{\stackrel{(5.2)}{ }} & \mathcal{R}\left(\mathrm{GL}_{n}(\mathbb{C})\right)
\end{array}
$$




\section{$\S 6$ Holomorphic reflexivity as a generalization of Pontryagin duality}

\section{(a) Pontryagin duality for compactly generated Stein groups}

The complex circle $\mathbb{C}^{\times}$we were talking about in $\S 3(\mathrm{a})$ occupies among all Abelian compactly generated Stein groups the same place, as the usual "real" circle $\mathbb{T}=\mathbb{R} / \mathbb{Z}$ among all locally compact Abelian groups (or among all Abelian compactly generated real Lie groups), since for the Abelian compactly generated Stein groups the following variant of Pontryagin's duality theory holds.

Let $G$ be an Abelian compactly generated Stein group. Let us call an arbitrary holomorphic homomorphism of a Stein group $G$ into the complex circle

$$
\chi \in G^{\bullet} \Longleftrightarrow \chi: G \rightarrow \mathbb{C}^{\times}
$$

a holomorphic character on $G$. The set $G^{\bullet}$ of all holomorphic characters on $G$ is a topological group with respect to the pointwise multiplication and the topology of uniform convergence on compact sets. The following theorem shows that the operation $G \mapsto G^{\bullet}$ is analogous to the Pontryagin operation of passage to the dual locally compact Abelian group:

Theorem 6.1. If $G$ is an Abelian compactly generated Stein group, then its dual group $G^{\bullet}$ is again an Abelian compactly generated group and the mapping

$$
\mathrm{i}_{G}: G \rightarrow G^{\bullet \bullet}, \quad \mathrm{i}_{G}(x)(\chi)=\chi(x), \quad x \in G, \chi \in G^{\bullet}
$$

is an isomorphism of (topological groups and of) functors $G \mapsto G$ and $G \mapsto G^{\bullet \bullet}$ :

$$
G^{\bullet \bullet} \cong G
$$

In view of this fact we call $G^{\bullet}$ dual complex group for the group $G$.

Proof. First we need to note that this is true for the special cases where $G=\mathbb{C}, \mathbb{C}^{\times}, \mathbb{Z}$ and for the case of a finite Abelian group $G=F$. This is a corollary of the following obvious formulas:

$$
\mathbb{C}^{\bullet} \cong \mathbb{C}, \quad\left(\mathbb{C}^{\times}\right)^{\bullet} \cong \mathbb{Z}, \quad \mathbb{Z}^{\bullet} \cong \mathbb{C}^{\times}, \quad F^{\bullet} \cong F
$$

After that it remains to note that every compactly generated Stein group has the form

$$
G \cong \mathbb{C}^{l} \times\left(\mathbb{C}^{\times}\right)^{m} \times \mathbb{Z}^{n} \times F \quad\left(l, m, n \in \mathbb{Z}_{+}\right)
$$

so its dual group has the form

$$
G^{\bullet} \cong \mathbb{C}^{l} \times \mathbb{Z}^{m} \times\left(\mathbb{C}^{\times}\right)^{n} \times F \quad\left(l, m, n \in \mathbb{Z}_{+}\right)
$$

So $G^{\bullet}$ is an Abelian compactly generated Stein group. The second dual group $G^{\bullet \bullet}$ turns out to be isomorphic to $G$ :

$$
G^{\bullet \bullet} \cong \mathbb{C}^{l} \times\left(\mathbb{C}^{\times}\right)^{m} \times \mathbb{Z}^{n} \times F \cong G
$$

\section{(b) Fourier transform as an Arens-Michael envelope}

If $G$ is an Abelian compactly generated Stein group, then every its holomorphic character $\chi: G \rightarrow \mathbb{C}^{\times}$is a holomorphic function on $G$. In other words we can think of the dual complex group $G^{\bullet}$ as a subgroup in the group of invertible elements of the algebra $\mathcal{O}(G)$ of holomorphic functions on $G$ :

$$
G^{\bullet} \subset \mathcal{O}(G)
$$


If we pass to dual objects by Theorem 6.1 we obtain that the group $G$ itself is included by the transformation $\mathrm{i}_{G}$ into the group of invertible elements of the algebra $\mathcal{O}\left(G^{\bullet}\right)$ of holomorphic functions on $G^{\bullet}:$

$$
\mathrm{i}_{G}: G \rightarrow G^{\bullet \bullet} \subset \mathcal{O}\left(G^{\bullet}\right) .
$$

On the other hand, obviously, $G$ is included (through delta-functionals) into algebra $\mathcal{O}^{\star}(G)$ :

$$
\delta: G \rightarrow \mathcal{O}^{\star}(G) \quad\left(x \mapsto \delta^{x}\right) .
$$

By [1, Theorem 10.12] this implies that there exists a unique homomorphism of stereotype algebras

$$
\sharp_{G}: \mathcal{O}^{\star}(G) \rightarrow \mathcal{O}\left(G^{\bullet}\right),
$$

such that the following diagram is commutative:

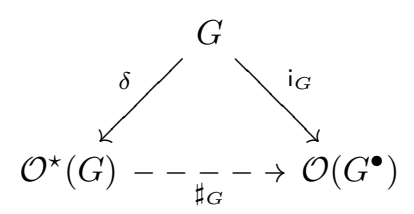

(this is the property of being group algebra for $\mathcal{O}^{\star}(G)$ ). It is natural to call the homomorphism $\sharp_{G}$ : $\mathcal{O}^{\star}(G) \rightarrow \mathcal{O}\left(G^{\bullet}\right)$ the (inverse) Fourier transform on the Stein group $G$, since it is defined by the same formula as for the (inverse) Fourier transform for measures and distributions [16, 31.2]:

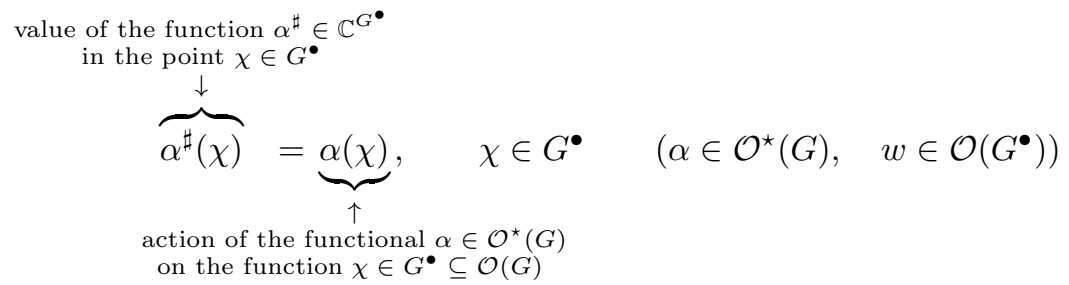

Theorem 6.2. For any Abelian compactly generated Stein group $G$ its Fourier transform

$$
\sharp_{G}: \mathcal{O}^{\star}(G) \rightarrow \mathcal{O}\left(G^{\bullet}\right),
$$

is:

(a) a homomorphism of rigid Hopf-Fréchet algebras, and

(b) an Arens-Michael envelope of the algebra $\mathcal{O}^{\star}(G)$.

As a corollary the following isomorphisms of rigid Hopf-Fréchet algebras hold:

$$
\mathcal{O}_{\text {exp }}^{\star}(G) \cong\left(\mathcal{O}^{\star}(G)\right)^{\complement} \cong \mathcal{O}\left(G^{\bullet}\right)
$$

and the reflexivity diagram for $G$ takes the form

$$
\begin{aligned}
& \mathcal{O}^{\star}(G) \quad \stackrel{\text { Fourier transform }}{\stackrel{\text { 6.1 }}{\longmapsto}} \mathcal{O}\left(G^{\bullet}\right) \\
& \text { *1 I* } \\
& \mathcal{O}(G) \stackrel{\text { Fourier transform }}{\stackrel{\sqrt{6.1}}{\stackrel{1}{*}}} \quad \mathcal{O}^{\star}\left(G^{\bullet}\right)
\end{aligned}
$$

Like Theorem 6.1, this is proved by successive consideration of the cases $G=\mathbb{C}, \mathbb{C}^{\times}, \mathbb{Z}$ and the case of arbitrary finite Abelian group $G=F$. In the rest of this section up to the "inclusion diagram" we devote to this. 
Finite Abelian group. As we told in $\S 3($ a) , every finite group $G$ can be considered as a complex Lie group (of zero dimension), on which every function is holomorphic. Moreover, in example §4 (c) we have noticed that every function on $G$ has exponential type, so the algebras $\mathcal{O}_{\exp }(G), \mathcal{O}(G)$ and $\mathbb{C}^{G}$ coincide:

$$
\mathcal{O}_{\exp }(G)=\mathcal{O}(G)=\mathbb{C}^{G}
$$

If in addition $G$ is commutative, then the theorem 6.2 we illustrate here turns into a formally more strong proposition:

Proposition 6.1. If $G$ is a finite Abelian group, then the formula (6.1) establishes an isomorphism of Hopf algebras:

$$
\mathcal{O}_{\text {exp }}^{\star}(G)=\mathcal{O}^{\star}(G)=\mathbb{C}_{G} \cong \mathbb{C}^{G^{\bullet}}=\mathcal{O}\left(G^{\bullet}\right)=\mathcal{O}_{\exp }\left(G^{\bullet}\right)
$$

Complex plane $\mathbb{C}$. Let for every $\lambda \in \mathbb{C}$ the symbol $\chi_{\lambda}$ denote a character on the group $\mathbb{C}$, defined by formula:

$$
\chi_{\lambda}(t)=e^{\lambda \cdot t}
$$

The mapping $\lambda \in \mathbb{C} \mapsto \chi_{\lambda} \in \mathbb{C}^{\bullet}$ is an isomorphism of complex groups

$$
\mathbb{C} \cong \mathbb{C}^{\bullet}
$$

and this isomorphism turns formula (6.1) into formula

$$
\alpha^{\sharp}(\lambda)=\alpha\left(\chi_{\lambda}\right), \quad \lambda \in \mathbb{C} \quad\left(\alpha \in \mathcal{O}_{\text {exp }}^{\star}(\mathbb{C}), \quad w \in \mathcal{O}(\mathbb{C})\right)
$$

(we denote this isomorphism by the same symbol $\sharp$, although formally it is a composition of mappings (6.1) and $\lambda \mapsto \chi_{\lambda}$ ). As a result Theorem 6.2 being applied to the group $G=\mathbb{C}$ is turned into

Proposition 6.2. Formula (6.5) defines a homomorphism of stereotype Hopf algebras

$$
\sharp_{\mathbb{C}}: \mathcal{O}^{\star}(\mathbb{C}) \rightarrow \mathcal{O}(\mathbb{C})
$$

which is an Arens-Michael envelope of the algebra $\mathcal{O}^{\star}(\mathbb{C})$, and establishes an isomorphism of Hopf-Fréchet algebras:

$$
\mathcal{O}_{\exp }^{\star}(\mathbb{C}) \cong \mathcal{O}(\mathbb{C})
$$

We shall need the following

Lemma 6.1. Seminorms of the form

$$
\|\alpha\|_{C}=\sum_{k \in \mathbb{N}}\left|\alpha_{k}\right| \cdot C^{k}, \quad C \geqslant 0
$$

(i.e. special case of seminorms (3.73), when $r_{k}=\frac{C^{k}}{k !}$ ) form a fundamental system in the set of all submultiplicative continuous seminorms on $\mathcal{O}^{\star}(\mathbb{C})$.

Proof. As we had noted in $\S 3(\mathrm{c})$ the multiplication in $\mathcal{O}(\mathbb{C})$ and in $\mathcal{O}^{\star}(\mathbb{C})$ is defined by the same formulas on series (3.71). So we can say that the submultiplicativity of seminorms (6.7) is already proven, since in Example 5.3 we had proven the same fact for seminorms (3.72), defined by the same formula on the series.

Let us show that seminorms (6.7) form a fundamental system among all submultiplicative continuous seminorms on $\mathcal{O}^{\star}(\mathbb{C})$. This is done like in Proposition 3.14. Let $p$ be a submultiplicative continuous seminorm:

$$
p(\alpha * \beta) \leqslant p(\alpha) \cdot p(\beta)
$$

Put

$$
r_{k}=\frac{1}{k !} p\left(\zeta_{k}\right)
$$


Then

$$
(k+l) ! \cdot r_{k+l}=p\left(\zeta_{k+l}\right)=p\left(\zeta_{k} * \zeta_{l}\right) \leqslant p\left(\zeta_{k}\right) \cdot p\left(\zeta_{l}\right)=\left(k ! \cdot r_{k}\right) \cdot\left(l ! \cdot r_{l}\right)
$$

The sequence $A_{k}=r_{k} \cdot k$ ! satisfies the recurrent inequality $A_{k+1} \leqslant A_{k} \cdot A_{1}$, which implies $A_{k} \leqslant C^{k}$, for $C=A_{1}$. This in its turn implies inequalities

$$
r_{k} \leqslant \frac{C^{k}}{k !}
$$

Now using the same reasonings as in the proof of Proposition 3.14 we obtain:

$$
p(\alpha) \leqslant(\underline{3.76}) \leqslant|||\alpha|||_{r}=\sum_{k \in \mathbb{N}} r_{k} \cdot\left|\alpha_{k}\right| \cdot k ! \leqslant \sum_{k \in \mathbb{N}}\left|\alpha_{k}\right| \cdot C^{k}=\|\alpha\|_{C}
$$

Proof of Proposition 6.2. Note from the very beginning that the mapping $\sharp_{\mathbb{C}}: \mathcal{O}^{\star}(\mathbb{C}) \rightarrow \mathcal{O}(\mathbb{C})$, defined by Formula (6.5) is continuous: by the continuity of the mapping $\lambda \in \mathbb{C} \mapsto \chi_{\lambda} \in \mathcal{O}(\mathbb{C})$, every compact set $T$ in $\mathbb{C}$ is turned into a compact set $\left\{\chi_{\lambda} ; \lambda \in T\right\}$ in $\mathcal{O}(\mathbb{C})$, so if the net of functionals $\alpha_{i}$ tends to zero in $\mathcal{O}^{\star}(\mathbb{C})$, then for any compact set $T$ in $\mathbb{C}$ we have

$$
\alpha_{i}^{\sharp}(\lambda)=\alpha_{i}\left(\chi_{\lambda}\right) \underset{\lambda \in T}{\rightrightarrows} 0, \quad i \rightarrow \infty
$$

Thus, the functions $\alpha_{i}^{\sharp}$ tend to zero in $\mathcal{O}(\mathbb{C})$.

Further, note that the mapping $\sharp_{\mathbb{C}}$ turns the functionals $\zeta_{n}$ into functions $z^{n}$ :

$$
\left(\zeta_{n}\right)^{\sharp}(\lambda)=\zeta_{n}\left(\chi_{\lambda}\right)=\left.\left(\frac{\mathrm{d}^{n}}{\mathrm{~d} t^{n}} e^{\lambda t}\right)\right|_{t=0}=\lambda^{n}=z^{n}(\lambda)
$$

From this and from the continuity of $\sharp_{\mathbb{C}}$ it follows that the mapping act on functionals $\alpha$ as the substitution of monomials $\zeta_{n}$ in the decomposition (3.70) by monomials $z^{n}$ :

$$
\alpha^{\sharp}=\left(\sum_{n=0}^{\infty} \alpha_{n} \cdot \zeta_{n}\right)^{\sharp}=\sum_{n=0}^{\infty} \alpha_{n} \cdot\left(\zeta_{n}\right)^{\sharp}=\sum_{n=0}^{\infty} \alpha_{n} \cdot z^{n}
$$

This immediately implies the rest.

1. First, the mapping $\sharp_{\mathbb{C}}: \mathcal{O}^{\star}(\mathbb{C}) \rightarrow \mathcal{O}(\mathbb{C})$ is an Arens-Michael envelope, since by Lemma 6.1, every submultiplicative continuous seminorm on $\mathcal{O}^{\star}(\mathbb{C})$ is majorized by a seminorm of the form (6.7), which in its turn can be extended by the mapping $\sharp_{\mathbb{C}}$ to a seminorm (3.72) on $\mathcal{O}(\mathbb{C})$.

2. Second, the mapping $\sharp_{\mathbb{C}}: \mathcal{O}^{\star}(\mathbb{C}) \rightarrow \mathcal{O}(\mathbb{C})$ is a homomorphism of algebras, since by formulas (3.69)(3.70) these algebras can be considered as algebras of power series, where the multiplication is defined by usual formulas for power series (3.71), and $\sharp_{\mathbb{C}}$ will be just inclusion of one algebra into another, more wide, algebra.

3. To prove that the mapping $\sharp_{\mathbb{C}}: \mathcal{O}^{\star}(\mathbb{C}) \rightarrow \mathcal{O}(\mathbb{C})$ is an isomorphism of coalgebras, let us note that the dual mapping

$$
(\sharp \mathbb{C})^{\star}: \mathcal{O}^{\star}(\mathbb{C}) \rightarrow\left(\mathcal{O}^{\star}(\mathbb{C})\right)^{\star}=\mathcal{O}(\mathbb{C})^{\star \star}
$$

coincides with $\sharp \mathbb{C}$ up to the isomorphism $i_{\mathcal{O}(\mathbb{C})}: \mathcal{O}(\mathbb{C}) \cong \mathcal{O}(\mathbb{C})^{\star \star}$ :

$$
\mathcal{O}^{\star}(\mathbb{C}) \underset{\substack{\left(\sharp_{C}\right)^{\star}}}{\longrightarrow} \mathcal{O}(\mathbb{C})^{\star \star}
$$


This follows from the formula

$$
\sharp_{\mathbb{C}}\left(\delta^{x}\right)=\chi_{x}
$$

Indeed,

$$
\sharp_{\mathbb{C}}\left(\delta^{x}\right)(\lambda)=\delta^{x}\left(\chi_{\lambda}\right)=\chi_{\lambda}(x)=e^{\lambda x}=\chi_{x}(\lambda)
$$

Now we obtain:

$\left.\sharp^{\star}\left(\delta^{a}\right)\left(\delta^{b}\right)=\delta^{a}\left(\sharp_{\mathbb{C}}\left(\delta^{b}\right)\right)=\underline{6.9}\right)=\delta^{a}\left(\chi_{b}\right)=\sharp_{\mathbb{C}}\left(\delta^{a}\right)(b)=\delta^{b}\left(\sharp_{\mathbb{C}}\left(\delta^{a}\right)\right)==\mathrm{i}_{\mathcal{O}(\mathbb{C})}\left(\sharp_{\mathbb{C}}\left(\delta^{a}\right)\right)\left(\delta^{b}\right)=\left(\mathrm{i}_{\mathcal{O}}(\mathbb{C}) \circ \sharp\right)\left(\delta^{a}\right)\left(\delta^{b}\right)$

This is true for all $a, b \in \mathbb{C}$. On the other hand, the linear hull of delta-functionals is dense in $\mathcal{O}^{\star}$, so

$$
\left(\sharp_{\mathbb{C}}\right)^{\star}=i_{\mathcal{O}(\mathbb{C})} \circ \sharp_{\mathbb{C}}
$$

i.e. the diagram (6.8) is commutative. But we have already proved that $\sharp_{\mathbb{C}}$ is a homomorphism of algebras, and for $i_{\mathcal{O}(\mathbb{C})}$ this is obvious. Hence, $\left(\sharp_{\mathbb{C}}\right)^{\star}$ is also a homomorphism of algebras, and this means that $\sharp_{\mathbb{C}}$ is a homomorphism of coalgebras.

4. Now it remains to prove that $\sharp_{\mathbb{C}}$ preserves antipode:

$$
\begin{gathered}
\sigma_{\mathcal{O}(\mathbb{C})}\left(\chi_{\lambda}\right)(x)=\chi_{\lambda}(-x)=e^{-\lambda x}=\left(e^{\lambda x}\right)^{-1}=\chi_{\lambda}(x)^{-1} \\
\Downarrow \\
\sigma_{\mathcal{O}(\mathbb{C})}\left(\chi_{\lambda}\right)=\chi_{\lambda}^{-1} \\
\Downarrow \\
\left(\sigma_{\mathcal{O} *(\mathbb{C})}(\alpha)\right)^{\sharp}(\lambda)=\left(\sigma_{\mathcal{O} *(\mathbb{C})}(\alpha)\right)\left(\chi_{\lambda}\right)=\left(\alpha \circ \sigma_{\mathcal{O}(\mathbb{C})}\right)\left(\chi_{\lambda}\right)=\alpha\left(\sigma_{\mathcal{O}(\mathbb{C})}\left(\chi_{\lambda}\right)\right)=\alpha\left(\chi_{\lambda}^{-1}\right)=\alpha^{\sharp}(-\lambda)=\sigma_{\mathcal{O}(\mathbb{C})}\left(\alpha^{\sharp}\right)(\lambda) \\
\Downarrow \\
\left(\sigma_{\mathcal{O} *(\mathbb{C})}(\alpha)\right)^{\sharp}=\sigma_{\mathcal{O}(\mathbb{C})}\left(\alpha^{\sharp}\right)
\end{gathered}
$$

Complex circle $\mathbb{C}^{\times}$. Let for any $n \in \mathbb{Z}$ the symbol $z^{n}$ denote the character on the group $\mathbb{C}^{\times}$, defined by formula

$$
z^{n}(t)=t^{n}
$$

The mapping $n \in \mathbb{Z} \mapsto z^{n} \in\left(\mathbb{C}^{\times}\right)^{\bullet}$ is a homomorphism of complex groups

$$
\mathbb{Z} \cong\left(\mathbb{C}^{\times}\right)^{\bullet}
$$

and formula (6.1) under this isomorphism takes the form

$$
\alpha^{\sharp}(n)=\alpha\left(z^{n}\right), \quad n \in \mathbb{Z} \quad\left(\alpha \in \mathcal{O}_{\text {exp }}^{\star}\left(\mathbb{C}^{\times}\right)\right)
$$

(like in the previous example we denote this mapping by the same symbol $\sharp$, although formally this is a composition of mappings (6.1) and $n \mapsto z^{n}$ ). As a result Theorem 6.2. being applied to group $G=\mathbb{C}^{\times}$ is turned into

Proposition 6.3. Formula (6.10) defines a homomorphism of stereotype Hopf algebras

$$
\sharp_{\mathbb{C} \times}: \mathcal{O}^{\star}\left(\mathbb{C}^{\times}\right) \rightarrow \mathcal{O}(\mathbb{Z})
$$

which is an Arens-Michael envelope of the algebra $\mathcal{O}^{\star}\left(\mathbb{C}^{\times}\right)$, and establishes an isomorphism of HopfFréchet algebras

$$
\mathcal{O}_{\text {exp }}^{\star}\left(\mathbb{C}^{\times}\right) \cong \mathcal{O}(\mathbb{Z})=\mathbb{C}^{\mathbb{Z}}
$$

We shall need 
Lemma 6.2. The seminorms of the form

$$
\|\alpha\|_{N}=\sum_{|n| \leqslant N}\left|\alpha_{n}\right|, \quad N \in \mathbb{N}
$$

- i.e. the special case of seminorms (3.48), when

$$
r_{n}= \begin{cases}1, & |n| \leqslant N \\ 0, & |n|>N\end{cases}
$$

- form a fundamental system in the set of all submultiplicative continuous seminorms on $\mathcal{O}^{\star}\left(\mathbb{C}^{\times}\right)$.

Proof. Submultiplicativity of seminorms (6.12) follows from the formula for the operation of multiplication in $\mathcal{O}^{\star}\left(\mathbb{C}^{\times}\right)$:

$$
\left\|\left.\alpha * \beta\right|_{N}=\sum_{|n| \leqslant N}\left|(\alpha * \beta)_{n}\right|=(\underline{3.46})=\sum_{|n| \leqslant N}\left|\alpha_{n} \cdot \beta_{n}\right| \leqslant\left(\sum_{|n| \leqslant N}\left|\alpha_{n}\right|\right) \cdot\left(\sum_{|n| \leqslant N}\left|\beta_{n}\right|\right)=\right\| \alpha\left\|_{N} \cdot\right\| \beta \|_{N}
$$

Let us show that seminorms (3.48) form a fundamental system among all submultiplicative continuous seminorms on $\mathcal{O}^{\star}\left(\mathbb{C}^{\times}\right)$. Let $p$ be a submultiplicative continuous seminorm:

$$
p(\alpha * \beta) \leqslant p(\alpha) \cdot p(\beta)
$$

Put

$$
r_{n}=p\left(\zeta_{n}\right)
$$

Then

$$
r_{n}=p\left(\zeta_{n}\right)=p\left(\zeta_{n} * \zeta_{n}\right) \leqslant p\left(\zeta_{n}\right) \cdot p\left(\zeta_{n}\right)=r_{n}^{2}
$$

i.e. $0 \leqslant r_{n} \leqslant r_{n}^{2}$, hence $r_{n} \geqslant 1$, or $r_{n}=0$. But by Lemma 3.2, the numbers $r_{n}$ must satisfy the condition (3.49), which in its turn implies that $r_{n} \rightarrow 0$. This is possible only if all those numbers, except maybe a finite subfamily, vanishes:

$$
\exists N \in \mathbb{N} \quad \forall n \in \mathbb{Z} \quad|n|>N \quad \Longrightarrow \quad r_{n}=0
$$

Put $M=\max _{n} r_{n}$, then by Lemma 3.2 we obtain:

$$
p(\alpha) \leqslant\left\||| \alpha||_{r}=\sum_{n \in \mathbb{Z}} r_{n} \cdot\left|\alpha_{n}\right|=\sum_{|n| \leqslant N} r_{n} \cdot\left|\alpha_{n}\right| \leqslant \sum_{|n| \leqslant N} M \cdot\left|\alpha_{n}\right|=M \cdot\right\| \alpha \|_{N}
$$

Beginning of the proof of Proposition 6.3. Note that the mapping $\sharp \mathbb{C}^{\times}: \mathcal{O}^{\star}\left(\mathbb{C}^{\times}\right) \rightarrow \mathcal{O}(\mathbb{Z})$, defined by Formula (6.10) is continuous: if a net of functionals $\alpha_{i}$ tends to zero in $\mathcal{O}^{\star}\left(\mathbb{C}^{\times}\right)$, then for any $n \in \mathbb{Z}$ we have

$$
\alpha_{i}^{\sharp}(n)=\alpha_{i}\left(z^{n}\right) \longrightarrow 0, \quad i \rightarrow \infty
$$

This means that $\alpha_{i}^{\sharp}$ tends to zero in $\mathcal{O}(\mathbb{Z})=\mathbb{C}^{\mathbb{Z}}$. in $\mathbb{Z}$ :

Further, let us note that the mapping $\mathbb{E}_{\mathbb{C} \times}$ turns functionals $\zeta_{k}$ into characteristic functions of singletons

$$
\left(\zeta_{k}\right)^{\sharp}(n)=\zeta_{k}\left(z^{n}\right)=(3.32)=\left\{\begin{array}{ll}
1, & n=k \\
0, & n \neq k
\end{array}\right\}=(3.16)=1_{k}(n)
$$

From this and from the continuity of $\sharp_{\mathbb{C} \times}$ it follows that this mapping acts on functionals $\alpha$ as substitution of monomials $\zeta_{n}$ in the decomposition (3.70) by monomials $1_{n}$ :

$$
\alpha^{\sharp}=\left(\sum_{n \in \mathbb{Z}} \alpha_{n} \cdot \zeta_{n}\right)^{\sharp}=\sum_{n \in \mathbb{Z}} \alpha_{n} \cdot\left(\zeta_{n}\right)^{\sharp}=\sum_{n \in \mathbb{Z}} \alpha_{n} \cdot 1_{n}
$$


This in its turn imply the most part of Proposition 6.3

1. First, the mapping $\sharp_{\mathbb{C}^{\times}}: \mathcal{O}^{\star}\left(\mathbb{C}^{\times}\right) \rightarrow \mathcal{O}(\mathbb{Z})$ is an Arens-Michael envelope, so by Lemma 6.2, every submultiplicative continuous seminorm on $\mathcal{O}^{\star}\left(\mathbb{C}^{\times}\right)$is majorized by a seminorm of the form (6.12), which in its turn can be extended by the mapping $\sharp_{\mathbb{C}}$ to a continuous seminorm on $\mathcal{O}(\mathbb{Z})=\mathbb{C}^{\mathbb{Z}}$.

2. Second, the mapping $\sharp_{\mathbb{C}^{\times}}: \mathcal{O}^{\star}\left(\mathbb{C}^{\times}\right) \rightarrow \mathcal{O}(\mathbb{Z})$ is a homomorphism of algebras, since by the formula for multiplication (3.46) in $\mathcal{O}^{\star}\left(\mathbb{C}^{\times}\right)$, this mapping can be represented as a space of two-sided sequences $\alpha_{n}$ with the coordinate-wise multiplication, which is included in the wider space $\mathcal{O}(\mathbb{Z})=\mathbb{C}^{\mathbb{Z}}$ of all two-sided sequences with the coordinate-wise multiplication by the mapping $\sharp_{\mathbb{C}^{\times}}$.

3. we have to postpone to the next example (at page 104) the proof of the fact that the mapping $\sharp \mathbb{C}^{\times}: \mathcal{O}^{\star}\left(\mathbb{C}^{\times}\right) \rightarrow \mathcal{O}(\mathbb{Z})$ is an isomorphism of coalgebras.

4. Let us check that $\sharp_{\mathbb{C}^{\times}}$preserves antipode:

$$
\begin{gathered}
\sigma_{\mathcal{O}\left(\mathbb{C}^{\times}\right)}\left(z^{n}\right)(x)=z^{n}\left(x^{-1}\right)=x^{-n}=z^{-n}(x) \\
\Downarrow \\
\sigma_{\mathcal{O}\left(\mathbb{C}^{\times}\right)}\left(z^{n}\right)=z^{-n} \\
\Downarrow \\
\left(\sigma_{\mathcal{O}^{\star}\left(\mathbb{C}^{\times}\right)}(\alpha)\right)^{\sharp}(n)=\left(\sigma_{\mathcal{O}^{\star}\left(\mathbb{C}^{\times}\right)}(\alpha)\right)\left(z^{n}\right)=\left(\alpha \circ \sigma_{\mathcal{O}\left(\mathbb{C}^{\times}\right)}\right)\left(z^{n}\right)=\alpha\left(\sigma_{\mathbb{C}^{\times}}\left(z^{n}\right)\right)=\alpha\left(z^{-n}\right)=\alpha^{\sharp}(-n)=\sigma_{\mathcal{O}(\mathbb{Z})}\left(\alpha^{\sharp}\right)(n) \\
\Downarrow \\
\left(\sigma_{\mathcal{O}^{\star}\left(\mathbb{C}^{\times}\right)}(\alpha)\right)^{\sharp}=\sigma_{\mathcal{O}(\mathbb{Z})}\left(\alpha^{\sharp}\right)
\end{gathered}
$$

Group of integers $\mathbb{Z}$. Let for any $t \in \mathbb{C}^{\times}$the symbol $\chi_{t}$ denote a character on the group $\mathbb{Z}$, defined by formula

$$
\chi_{t}(n)=t^{n}
$$

The mapping $t \in \mathbb{C}^{\times} \mapsto \chi_{t} \in \mathbb{Z}^{\bullet}$ is an isomorphism of complex group

$$
\mathbb{C}^{\times} \cong \mathbb{Z}^{\bullet}
$$

and Formula (6.1) under this isomorphism takes the form

$$
\alpha^{\sharp}(t)=\alpha\left(\chi_{t}\right), \quad t \in \mathbb{C}^{\times} \quad\left(\alpha \in \mathcal{O}_{\text {exp }}^{\star}(\mathbb{Z})\right)
$$

(again we denote this mapping by the same symbol $\sharp$, although it is a composition of the mappings (6.1) and $t \mapsto \chi_{t}$ ). As a result, Theorem 6.2, being applied to group $G=\mathbb{Z}$, is turned into

Proposition 6.4. Formula (6.14) defines a homomorphism of rigid stereotype Hopf algebras

$$
\sharp \mathbb{Z}: \mathcal{O}^{\star}(\mathbb{Z}) \rightarrow \mathcal{O}\left(\mathbb{C}^{\times}\right)
$$

which is an Arens-Michael envelope of the algebra $\mathcal{O}^{\star}(\mathbb{Z})$, and establishes an isomorphism of Hopf-Fréchet algebras

$$
\mathcal{O}_{\exp }^{\star}(\mathbb{Z}) \cong \mathcal{O}\left(\mathbb{C}^{\times}\right)
$$

We need

Lemma 6.3. The seminorms of the form

$$
\|\alpha\|_{C}=\sum_{n \in \mathbb{Z}}\left|\alpha_{n}\right| \cdot C^{|n|}, \quad C \geqslant 1
$$

(i.e. special case of seminorms (3.24), when $r_{n}=C^{|n|}$ ) form a fundamental system in the set of all submultiplicative continuous seminorms on $\mathcal{O}^{\star}(\mathbb{Z})$. 
Proof. We had already noted the submultiplicativity of seminorms (6.16) in Example 5.2, Let us show that they form a fundamental system. Let $p$ be a submultiplicative continuous seminorm on on $\mathcal{O}^{\star}(\mathbb{Z})$ :

$$
p(\alpha * \beta) \leqslant p(\alpha) \cdot p(\beta)
$$

Put $r_{n}=p\left(\delta^{n}\right)$. Then

$$
r_{k+l}=p\left(\delta^{k+l}\right)=p\left(\delta^{k} * \delta^{l}\right) \leqslant p\left(\delta^{k}\right) \cdot p\left(\delta^{l}\right)=r_{k} \cdot r_{l}
$$

From this recurrent formula it follows that

$$
r_{n} \leqslant M \cdot C^{|n|}
$$

where $M=r_{0}, C=\max \left\{r_{1} ; r_{-1}\right\}$, and now by Lemma 3.1 we obtain:

$$
p(\alpha) \leqslant(\underline{3.28}) \leqslant\left|\left\|\alpha \left|\left\|_{r}=\sum_{n \in \mathbb{Z}} r_{n} \cdot\left|\alpha_{n}\right| \leqslant \sum_{n \in \mathbb{Z}} M \cdot C^{|n|} \cdot\left|\alpha_{n}\right|=M \cdot\right\| \alpha \|_{C}\right.\right.\right.
$$

Proof of Proposition 6.4. First of all, let us note that the mapping $\sharp \mathbb{Z}: \mathcal{O}^{\star}(\mathbb{Z}) \rightarrow \mathcal{O}\left(\mathbb{C}^{\times}\right)$, defined by formula (6.14) is continuous: by continuity of the mapping $t \in \mathbb{C}^{\times} \mapsto \chi_{t} \in \mathcal{O}(\mathbb{Z})$, every compact set $T$ in $\mathbb{C}^{\times}$is turned into a compact set $\left\{\chi_{t} ; t \in T\right\}$ in $\mathcal{O}(\mathbb{Z})$, so if a net of functionals $\alpha_{i}$ turns to zero in $\mathcal{O}^{\star}(\mathbb{Z})$, then for any compact set $T$ in $\mathbb{C}^{\times}$we have

$$
\alpha_{i}^{\sharp}(t)=\alpha_{i}\left(\chi_{t}\right) \underset{\lambda \in T}{\rightrightarrows} 0, \quad i \rightarrow \infty
$$

This means that $\alpha_{i}^{\sharp}$ tends to zero in $\mathcal{O}\left(\mathbb{C}^{\times}\right)$.

Further, let us note that the mapping $\sharp_{\mathbb{Z}}$ turns functionals $\delta^{n}$ into monomials $z^{n}$ :

$$
\left(\delta^{n}\right)^{\sharp}(t)=\delta^{n}\left(\chi_{t}\right)=\chi_{t}(n)=t^{n}=z^{n}(t)
$$

This together with continuity of $\sharp_{\mathbb{Z}}$ implies that this mapping acts on functionals $\alpha$ as substitution of monomials $\delta^{n}$ in the decomposition (3.18) by monomials $z^{n}$ :

$$
\alpha^{\sharp}=\left(\sum_{n \in \mathbb{Z}} \alpha_{n} \cdot \delta^{n}\right)^{\sharp}=\sum_{n \in \mathbb{Z}} \alpha_{n} \cdot\left(\delta^{n}\right)^{\sharp}=\sum_{n \in \mathbb{Z}} \alpha_{n} \cdot z^{n}
$$

This implies the rest.

1. First, the mapping $\sharp_{\mathbb{Z}}: \mathcal{O}^{\star}(\mathbb{Z}) \rightarrow \mathcal{O}\left(\mathbb{C}^{\times}\right)$is an Arens-Michael envelope, so by Lemma 6.3, every submultiplicative seminorm on $\mathcal{O}^{\star}(\mathbb{Z})$ is majorized by a seminorm of the form (6.16), which in its turn can be extended by the mapping $\sharp_{\mathbb{Z}}$ to a seminorm (3.47) on $\mathcal{O}\left(\mathbb{C}^{\times}\right)$.

2. Second, the mapping $\sharp_{\mathbb{Z}}: \mathcal{O}^{\star}(\mathbb{Z}) \rightarrow \mathcal{O}\left(\mathbb{C}^{\times}\right)$is a homomorphism of algebras, since by (3.19)-(3.46) these are algebras of power series with the usual multiplication of power series, and $\sharp_{\mathbb{C}}$ is simply inclusion of one algebra into another, wider algebra.

3. To prove that the mapping $\sharp_{\mathbb{Z}}: \mathcal{O}^{\star}(\mathbb{Z}) \rightarrow \mathcal{O}\left(\mathbb{C}^{\times}\right)$is an isomorphism of coalgebras, let us note that the dual mapping

$$
\left(\sharp_{\mathbb{Z}}\right)^{\star}: \mathcal{O}^{\star}\left(\mathbb{C}^{\star}\right) \rightarrow\left(\mathcal{O}^{\star}(\mathbb{Z})\right)^{\star}=\mathcal{O}(\mathbb{Z})^{\star \star}
$$

up to the isomorphism $i_{\mathcal{O}(\mathbb{Z})}: \mathcal{O}(\mathbb{Z}) \cong \mathcal{O}(\mathbb{Z})^{\star \star}$ coincides with $\sharp_{\mathbb{C} \times}$ :

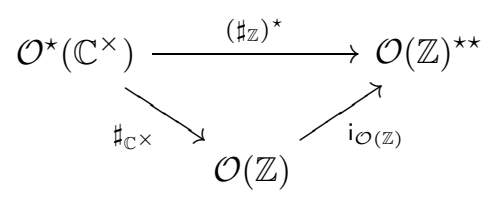


This follows from formula

$$
\delta^{t}\left(\sharp_{\mathbb{Z}}\left(\delta^{n}\right)\right)=t^{n}=\delta^{n}\left(\sharp_{\mathbb{C}^{\times}}\left(\delta^{t}\right)\right), \quad t \in \mathbb{C}^{\times}, \quad n \in \mathbb{Z}
$$

Indeed,

and

$$
\delta^{t}\left(\sharp_{\mathbb{Z}}\left(\delta^{n}\right)\right)=\sharp_{\mathbb{Z}}\left(\delta^{n}\right)(t)=\delta^{n}\left(\chi_{t}\right)=\chi_{t}(n)=t^{n}
$$

$$
\delta^{n}\left(\sharp_{\mathbb{C} \times}\left(\delta^{t}\right)\right)=\sharp_{\mathbb{C} \times}\left(\delta^{t}\right)(n)=\delta^{t}\left(z^{n}\right)=z^{n}(t)=t^{n}
$$

Now we obtain: for $t \in \mathbb{C}^{\times}$and $n \in \mathbb{Z}$

$$
\left(\sharp_{\mathbb{Z}}\right)^{\star}\left(\delta^{t}\right)\left(\delta^{n}\right)=\delta^{t}\left(\sharp_{\mathbb{Z}}\left(\delta^{n}\right)\right)=(\underline{6.18})=\delta^{n}\left(\sharp_{\mathbb{C} \times}\left(\delta^{t}\right)\right)=\mathrm{i}_{\mathcal{O}(\mathbb{Z})}\left(\sharp_{\mathbb{C} \times}\left(\delta^{t}\right)\right)\left(\delta^{n}\right)=\left(\mathrm{i}_{\mathcal{O}(\mathbb{Z})} \circ \sharp_{\mathbb{C} \times}\right)\left(\delta^{t}\right)\left(\delta^{n}\right)
$$

This is true for any $t \in \mathbb{C}^{\times}$and for any $n \in \mathbb{Z}$. On the other hand, delta-functionals generate a dense subspace in $\mathcal{O}^{\star}$, hence

$$
\left(\sharp_{\mathbb{Z}}\right)^{\star}=\mathrm{i}_{\mathcal{O}(\mathbb{Z})} \circ \sharp_{\mathbb{C} \times}
$$

i.e. diagram (6.17) is commutative. Now we can note that in the first part of proof of Proposition 6.3 (p 102) we have already verified that $\sharp_{\mathbb{C}} \times$ is a homomorphism of algebras. Certainly, the mapping $i_{\mathcal{O}(\mathbb{Z})}$ is also a homomorphism, so we conclude that $\left(\sharp_{\mathbb{Z}}\right)^{\star}$ is a homomorphism of algebras, and this means that $\sharp_{\mathbb{Z}}$ is a homomorphism of coalgebras.

4. Now it remains to check that $\sharp_{\mathbb{Z}}$ preserves antipode:

$$
\begin{gathered}
\sigma_{\mathcal{O}(\mathbb{Z})}\left(\chi_{t}\right)(n)=\chi_{t}(-n)=t^{-n}=\left(t^{-1}\right)^{n}=\chi_{t^{-1}}(n) \\
\Downarrow \\
\sigma_{\mathcal{O}(\mathbb{Z})}\left(\chi_{t}\right)=\chi_{t^{-1}} \\
\Downarrow \\
\left(\sigma_{\mathcal{O}^{\star}(\mathbb{Z})}(\alpha)\right)^{\sharp}(t)=\left(\sigma_{\mathcal{O}^{\star}(\mathbb{Z})}(\alpha)\right)\left(\chi_{t}\right)=\left(\alpha \circ \sigma_{\mathcal{O}(\mathbb{Z})}\right)\left(\chi_{t}\right)=\alpha\left(\sigma_{\mathcal{O}(\mathbb{Z})}\left(\chi_{t}\right)\right)=\alpha\left(\chi_{t^{-1}}\right)=\alpha^{\sharp}\left(t^{-1}\right)=\sigma_{\mathcal{O}\left(\mathbb{C}^{\times}\right)}\left(\alpha^{\sharp}\right)(t) \\
\Downarrow \\
\left(\sigma_{\mathcal{O}^{\star}(\mathbb{Z})}(\alpha)\right)^{\sharp}=\sigma_{\mathcal{O}\left(\mathbb{C}^{\times}\right)}\left(\alpha^{\sharp}\right)
\end{gathered}
$$

End of the proof of Proposition 6.3. In Proposition 6.3 it remains to proof that the mapping $\sharp \mathbb{C}^{\times}: \mathcal{O}^{\star}\left(\mathbb{C}^{\times}\right) \rightarrow$ $\mathcal{O}(\mathbb{Z})$ is an isomorphism of coalgebras. Note that the dual mapping

$$
\left(\sharp_{\mathbb{C} \times}\right)^{\star}: \mathcal{O}^{\star}(\mathbb{Z}) \rightarrow\left(\mathcal{O}^{\star}\left(\mathbb{C}^{\times}\right)\right)^{\star}=\mathcal{O}\left(\mathbb{C}^{\times}\right)^{\star \star}
$$

coincides with $\sharp_{\mathbb{Z}}$ up to the isomorphism $i_{\mathcal{O}\left(\mathbb{C}^{\times}\right)}: \mathcal{O}\left(\mathbb{C}^{\times}\right) \cong \mathcal{O}\left(\mathbb{C}^{\times}\right)^{\star \star}$ :

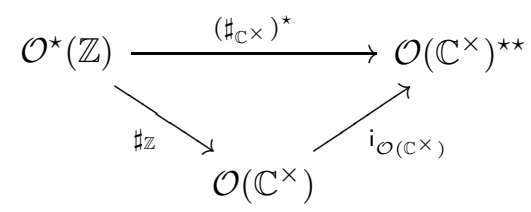

This also follows from (6.18): if $t \in \mathbb{C}^{\times}$and $n \in \mathbb{Z}$

$$
\left.\left(\sharp_{\mathbb{C}^{\times}}\right)^{\star}\left(\delta^{n}\right)\left(\delta^{t}\right)=\delta^{n}\left(\sharp_{\mathbb{C}^{\times}}\left(\delta^{t}\right)\right)=\underline{6.18}\right)=\delta^{t}\left(\sharp_{\mathbb{Z}}\left(\delta^{n}\right)\right)=\mathrm{i}_{\mathcal{O}\left(\mathbb{C}^{\times}\right)}\left(\sharp_{\mathbb{Z}}\left(\delta^{n}\right)\right)\left(\delta^{t}\right)=\left(\mathrm{i}_{\mathcal{O}(\mathbb{Z})} \circ \sharp_{\mathbb{C}^{\times}}\right)\left(\delta^{n}\right)\left(\delta^{t}\right)
$$

This is true for all $t \in \mathbb{C}^{\times}$and $n \in \mathbb{Z}$, and the delta-functionals form a dense subspace in $\mathcal{O}^{\star}$, so

$$
\left(\sharp_{\mathbb{C} \times}\right)^{\star}=\mathrm{i}_{\mathcal{O}\left(\mathbb{C}^{\times}\right)} \circ \sharp_{\mathbb{Z}}
$$

i.e. Diagram (6.19) is commutative. Now we can note that in proof of Proposition 6.4 we have already verified that $\sharp_{\mathbb{Z}}$ is a homomorphism of algebras. And for $i_{\mathcal{O}\left(\mathbb{C}^{\times}\right)}$this is obvious, so we obtain that $\left(\sharp_{\mathbb{C} \times}\right)^{\star}$ is also a homomorphism of algebras, and this means that $\sharp_{\mathbb{C}} \times$ is a homomorphism of coalgebras. 
Proof of Theorem 6.2 Now formulas (6.4), (6.6), (6.11), (6.15) prove isomorphism (6.2) for that cases $G=\mathbb{C}, \mathbb{C}^{\times}, \mathbb{Z}$ and for the case of finite group $G$, so it remains just to apply formulas (4.43) and (4.44): let us decompose an Abelian compactly generated Stein group $G$ into a direct product

$$
G=\mathbb{C}^{l} \times\left(\mathbb{C}^{\times}\right)^{m} \times \mathbb{Z}^{n} \times F
$$

where $F$ is a finite group. Then we have:

$$
\begin{aligned}
\mathcal{O}_{\text {exp }}^{\star}(G)=\mathcal{O}_{\text {exp }}^{\star}\left(\mathbb{C}^{l} \times\left(\mathbb{C}^{\times}\right)^{m} \times \mathbb{Z}^{n} \times F\right)=(4.43) & = \\
= & \mathcal{O}_{\text {exp }}^{\star}(\mathbb{C})^{\odot l} \odot \mathcal{O}_{\text {exp }}^{\star}\left(\mathbb{C}^{\times}\right)^{\odot m} \odot \mathcal{O}_{\text {exp }}^{\star}(\mathbb{Z})^{\odot n} \odot \mathcal{O}_{\text {exp }}^{\star}(F)= \\
= & \mathcal{O}\left(\mathbb{C}^{\bullet}\right)^{\odot l} \odot \mathcal{O}\left(\left(\mathbb{C}^{\times}\right)^{\bullet}\right)^{\odot m} \odot \mathcal{O}\left(\mathbb{Z}^{\bullet}\right)^{\odot n} \odot \mathcal{O}\left(F^{\bullet}\right)= \\
& =\left(\underline{4.43}=\mathcal{O}\left(\left(\mathbb{C}^{\bullet}\right)^{l} \times\left(\left(\mathbb{C}^{\times}\right)^{\bullet} \times\left(\mathbb{Z}^{\bullet}\right)^{n} \times F^{\bullet}\right)=\mathcal{O}\left(G^{\bullet}\right)\right.\right.
\end{aligned}
$$

\section{(c) Inclusion diagram}

Theorem 6.3. the following construction is a generalization of the Pontryagin duality theory from the category of Abelian compactly generated Stein groups to the category of compactly generated Stein groups with the algebraic connected component of identity,

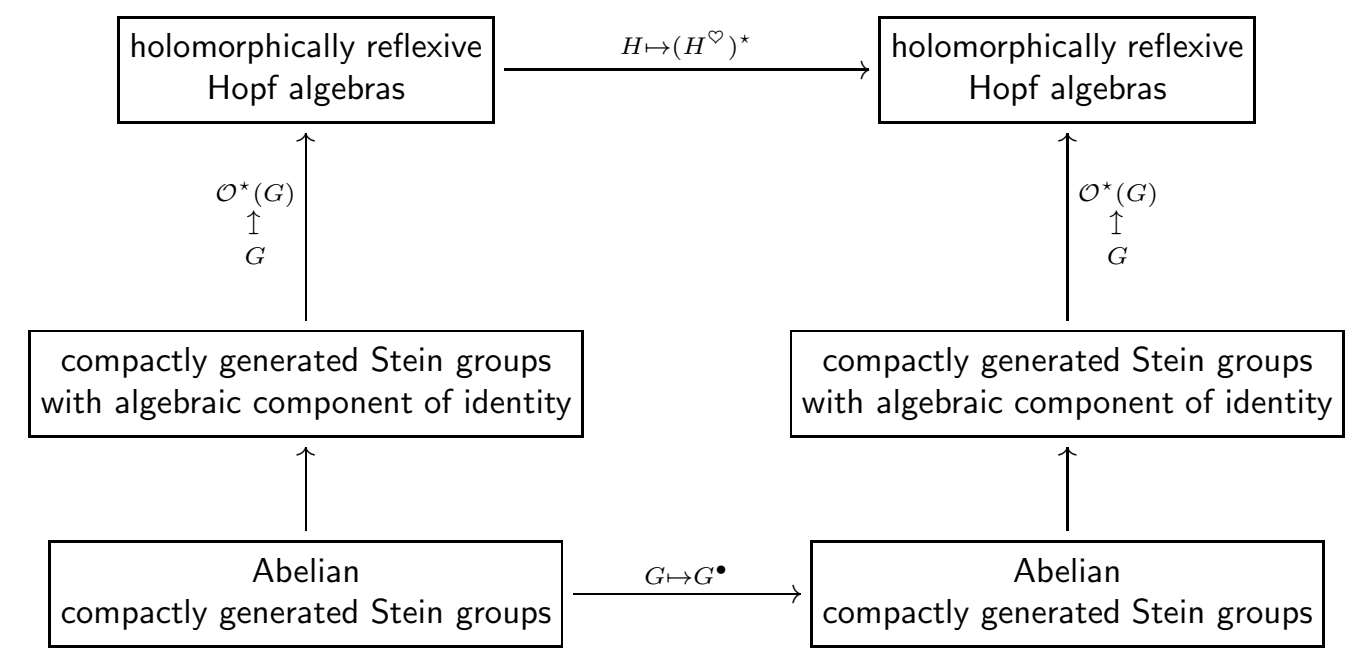

and the commutativity of this diagram is established by the isomorphism of functors

$$
\mathcal{O}^{\star}\left(G^{\bullet}\right) \cong\left(\left(\mathcal{O}^{\star}(G)\right)^{\complement}\right)^{\star}
$$

Proof. In Theorem 5.4 we have already showed that the functor $G \mapsto \mathcal{O}^{\star}(G)$ acts from the category of compactly generated Stein groups with algebraic component of identity into the category of holomorphically reflexive rigid stereotype Hopf algebras. So we need only to verify the commutativity of this "categorical diagram". This follows from Theorem 6.1] if $G$ is an Abelian compactly generated Stein group, then the walk around the diagram gives the following objects:

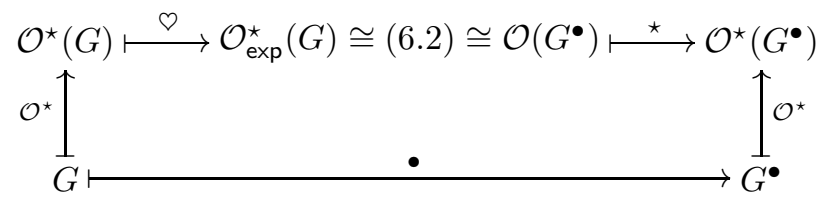




\section{$\S 7$ Appendix: holomorphic reflexivity of the quantum group ' $a z+b$ '}

In this final section we, following our promises in Introduction, will show by the example of the quantum group ' $a z+b$ ', that the holomorphic reflexivity described above does not restrict itself on algebras of analytical functionals $\mathcal{O}^{\star}(G)$, but lengthens into the theory of quantum groups.

\section{(a) Quantum combinatorial formulas}

The theory of quantum groups has its own analog of elementary combinatorics, used in the situations where the computations are applied to variables with the following commutation law

$$
y x=q x y
$$

where $q$ is a fixed number. For those computations in particular, the analogs of usual binomial formulas are deduced. Some of them will be useful for us in our constructions connected with ' $a z+b$ ', so we record them for further references (we refer the reader to details in C. Kassel's textbook [19]).

For an arbitrary positive integer $n$ we put

$$
(n)_{q}:=1+q+\ldots+q^{n-1}=\frac{q^{n}-1}{q-1}, \quad(n) !_{q}:=(1)_{q}(2)_{q} \ldots(n)_{q}=\frac{(q-1)\left(q^{2}-1\right) \ldots\left(q^{n}-1\right)}{(q-1)^{n}}
$$

The integer $(n) !_{q}$ will be called quantum factorial of the integer $n$. The quantum binomial coefficient is defined by formula

$$
\left(\begin{array}{l}
n \\
k
\end{array}\right)_{q}:= \begin{cases}\frac{(n) !_{q}}{(k) !_{q} \cdot(n-k) !_{q}}, & k \leqslant n \\
0, & k>n\end{cases}
$$

Theorem 7.1 (quantum binomial formula). Let $x$ and $y$ be elements of an associative algebra $A$ satisfying condition (7.1). Then for all $n \in \mathbb{N}$

$$
(x+y)^{n}=\sum_{k=0}^{n}\left(\begin{array}{l}
n \\
k
\end{array}\right)_{q} \cdot x^{k} \cdot y^{n-k}
$$

Theorem 7.2 (quantum Chu-Wandermond formula). For all $l, m, n \in \mathbb{N}$

$$
\begin{aligned}
\left(\begin{array}{c}
m+n \\
l
\end{array}\right)_{q}=\sum_{\max \{0, l-n\} \leqslant i \leqslant \min \{l, m\}} q^{(m-i) \cdot(l-i)} \cdot\left(\begin{array}{c}
m \\
i
\end{array}\right)_{q} \cdot\left(\begin{array}{c}
n \\
l-i
\end{array}\right)_{q}= & =\sum_{0 \leqslant i \leqslant l} q^{(m-i) \cdot(l-i)} \cdot\left(\begin{array}{c}
m \\
i
\end{array}\right)_{q} \cdot\left(\begin{array}{c}
n \\
l-i
\end{array}\right)_{q}
\end{aligned}
$$

Proof. ${ }^{6}$ If $l>m+n$, then for any $i=0, \ldots, m$ we have $n<l-m \leqslant l-i$, so $\left(\begin{array}{c}n \\ l-i\end{array}\right)_{q}=0$. Thus, both sums in (17.5) vanish. And the same happens with $\left(\begin{array}{c}m+n \\ l\end{array}\right)_{q}$, so formula (7.5) is trivial.

Thus only the case of $l \leqslant m+n$ is interesting. Consider the equality $(x+y)^{m+n}=(x+y)^{m}(x+y)^{n}$. Removing the parenthesis in (7.4) we obtain

$$
\sum_{l=0}^{m+n}\left(\begin{array}{c}
m+n \\
l
\end{array}\right)_{q} \cdot x^{l} \cdot y^{n-l}=\left(\sum_{i=0}^{m}\left(\begin{array}{c}
m \\
i
\end{array}\right)_{q} \cdot x^{i} \cdot y^{m-i}\right) \cdot\left(\sum_{j=0}^{n}\left(\begin{array}{c}
n \\
j
\end{array}\right)_{q} \cdot x^{j} \cdot y^{n-j}\right)=
$$

\footnotetext{
${ }^{6}$ We give here the proof of the Chu-Wandermond formula just to draw reader's attention to the limits of summing $\max \{0, l-n\} \leqslant i \leqslant \min \{l, m\}$, which will be useful below.
} 


$$
=\sum_{i=0}^{m} \sum_{j=0}^{n}\left(\begin{array}{c}
m \\
i
\end{array}\right)_{q} \cdot\left(\begin{array}{c}
n \\
j
\end{array}\right)_{q} \cdot x^{i} \cdot y^{m-i} \cdot x^{j} \cdot y^{n-j}=(7.1)=\sum_{i=0}^{m} \sum_{j=0}^{n}\left(\begin{array}{c}
m \\
i
\end{array}\right)_{q} \cdot\left(\begin{array}{c}
n \\
j
\end{array}\right)_{q} \cdot q^{(m-i) \cdot j} \cdot x^{i+j} \cdot y^{m+n-i-j}
$$

Let us consider in the last sum the terms with indices $i$ and $j$ connected by equality $i+j=l$. All those terms can be indexed by the parameter $i$, if we express $j$ trough $i$ by formula $j=l-i$. We need only to note that to obtain a bijection the index $i$ must vary in the following limits:

$$
\max \{0, l-n\} \leqslant i \leqslant \min \{l, m\}
$$

This follows from the restrictions on $i$ and $j$ :

$$
\left\{\begin{array} { l } 
{ 0 \leqslant i \leqslant m } \\
{ 0 \leqslant j \leqslant n }
\end{array} \Longleftrightarrow \left\{\begin{array} { l } 
{ 0 \leqslant i \leqslant m } \\
{ 0 \leqslant l - i \leqslant n }
\end{array} \Longleftrightarrow \left\{\begin{array} { l } 
{ 0 \leqslant i \leqslant m } \\
{ - n \leqslant i - l \leqslant 0 }
\end{array} \Longleftrightarrow \left\{\begin{array}{l}
0 \leqslant i \leqslant m \\
l-n \leqslant i \leqslant l
\end{array}\right.\right.\right.\right.
$$

Now equating the coefficients at monomial $x^{l} \cdot y^{n-l}$, we obtain the first equality in (7.5):

$$
\left(\begin{array}{c}
m+n \\
l
\end{array}\right)_{q} \sum_{\max \{0, l-n\} \leqslant i \leqslant \min \{l, m\}} q^{(m-i) \cdot(l-i)} \cdot\left(\begin{array}{c}
m \\
i
\end{array}\right)_{q} \cdot\left(\begin{array}{c}
n \\
l-i
\end{array}\right)_{q}
$$

The second equality is evident, since for $i<l-n$ and $i>l$ we have respectively $n<l-i$ or $l-i<0$, hence $\left(\begin{array}{c}n \\ l-i\end{array}\right)_{q}=0$, and the terms vanish:

$$
\sum_{\max \{0, l-n\} \leqslant i \leqslant \min \{l, m\}}=\underbrace{\sum_{0 \leqslant i<\max \{0, l-n\}}}_{0}+\sum_{\max \{0, l-n\} \leqslant i \leqslant \min \{l, m\}}+\underbrace{\sum_{\min \{l, m\}<i \leqslant m}}_{0}=\sum_{0 \leqslant i \leqslant m}
$$

\section{(b) Hopf algebra of skew polynomials and similar constructions}

Tensor products $X \odot \mathcal{R}(\mathbb{C}), X \circledast \mathcal{R}(\mathbb{C}), X \odot \mathcal{R}^{\star}(\mathbb{C}), X \circledast \mathcal{R}^{\star}(\mathbb{C})$

Theorem 7.3. Let $X$ be a stereotype space. Then

- the elements of tensor product $X \odot \mathcal{R}(\mathbb{C})$ are uniquely represented as converging series

$$
u=\sum_{k \in \mathbb{N}} u_{k} \odot t^{k}
$$

with coefficients $u_{k} \in X$ continuously depending on $u \in X \odot \mathcal{R}(\mathbb{C})$,

— the elements of tensor product $X \circledast \mathcal{R}(\mathbb{C})$ are uniquely represented as converging series

$$
u=\sum_{k \in \mathbb{N}} u_{k} \circledast t^{k}
$$

with coefficients $u_{k} \in X$ continuously depending on $u \in X \circledast \mathcal{R}(\mathbb{C})$,

- the elements of tensor product $X \odot \mathcal{R}^{\star}(\mathbb{C})$ are uniquely represented as converging series

$$
u=\sum_{k \in \mathbb{N}} u_{k} \odot \tau^{k}
$$

with coefficients $u_{k} \in X$ continuously depending on $u \in X \odot \mathcal{R}^{\star}(\mathbb{C})$,

— the elements of tensor product $X \circledast \mathcal{R}^{\star}(\mathbb{C})$ are uniquely represented as converging series

$$
u=\sum_{k \in \mathbb{N}} u_{k} \circledast \tau^{k}
$$

with coefficients $u_{k} \in X$ continuously depending on $u \in X \circledast \mathcal{R}^{\star}(\mathbb{C})$, 
Algebras of skew polynomials $A \stackrel{\varphi}{\odot} \mathcal{R}(\mathbb{C})$ and skew power series $A \stackrel{\varphi}{\circledast} \mathcal{R}^{\star}(\mathbb{C})$

Theorem 7.4. Let $A$ be an injective stereotype algebra and $\varphi: A \rightarrow A$ its (continuous) automorphism. Then the formula

$$
u \cdot v=\sum_{k \in \mathbb{N}} u_{k} \odot t^{k} \cdot \sum_{l \in \mathbb{N}} v_{l} \odot t^{l}=\sum_{n \in \mathbb{N}} \sum_{i=0}^{n} \underbrace{u_{i} \cdot \varphi^{i}\left(v_{n-i}\right)}_{\text {multiplication in } A} \odot t^{n}
$$

defines an associative and continuous multiplication on the tensor product $A \odot \mathcal{R}(\mathbb{C})$, and turns it into an injective stereotype algebra called algebra of skew polynomials (with respect to the automorphism $\varphi$ ) with coefficients in $A$ and is denoted by $A \stackrel{\varphi}{\odot} \mathcal{R}(\mathbb{C})$. If in addition $A$ is a Brauner algebra, then $A \stackrel{\varphi}{\odot} \mathcal{R}(\mathbb{C})$ is also a Brauner algebra.

Theorem 7.5. Let $A$ be a projective stereotype algebra and $\varphi: A \rightarrow A$ its (continuous) automorphism. Then the formula

$$
\alpha * \beta=\sum_{k \in \mathbb{N}} \alpha_{k} \circledast \tau^{k} * \sum_{l \in \mathbb{N}} \beta_{l} \circledast \tau^{l}=\sum_{n \in \mathbb{N}} \sum_{i=0}^{n} \underbrace{\alpha_{i} \cdot \varphi^{i}\left(\beta_{n-i)}\right)}_{\text {multiplication in } A} \circledast \tau^{n}
$$

defines an associative and continuous multiplication on the tensor product $A \circledast \mathcal{R}^{\star}(\mathbb{C})$, and turns it into a projective stereotype algebra called algebra of skew power series (with respect to the automorphism $\varphi$ ) with coefficients in $A$ and denoted by $A \stackrel{\varphi}{\circledast} \mathcal{R}^{\star}(\mathbb{C})$. If in addition $A$ is a Fréchet algebra, then $A \stackrel{\varphi}{\circledast} \mathcal{R}^{\star}(\mathbb{C})$ is also a Fréchet algebra.

Quantum pairs in stereotype Hopf algebras. Let $H$ ba an injective (resp., a projective) stereotype Hopf algebra and suppose we have

- a group-like central element $z$ in $H$

- a group-like central element $\omega$ in $H^{\star}$

Suppose in addition that the operators $\mathrm{M}_{\omega}^{\star}$ and $\mathrm{M}_{z}^{\star}$ dual to the operators of multiplication by elements $\omega$ and $z$, act on elements $z$ and $\omega$ as multiplication by a fixed number $q \in \mathbb{C} \backslash\{0\}$ :

$$
\mathrm{M}_{\omega}^{\star}(z)=q \cdot z, \quad \mathrm{M}_{z}^{\star}(\omega)=q \cdot \omega
$$

Then we call the pair $(z, \omega)$ a quantum pair in the Hopf algebra $H$ (with the parameter $q$ ).

The key examples for us are the pairs of algebras $\left\langle\mathcal{R}\left(\mathbb{C}^{\times}\right), \mathcal{R}^{\star}\left(\mathbb{C}^{\times}\right)\right\rangle$and $\left\langle\mathcal{O}\left(\mathbb{C}^{\times}\right), \mathcal{O}^{\star}\left(\mathbb{C}^{\times}\right)\right\rangle$on the complex circle $\mathbb{C}^{\times}$, we have considered in $\S 3(\mathrm{c})$. There by symbol $z$ we dented a monomial of power 1 on $\mathbb{C}^{\times}$:

$$
z(x):=x, \quad x \in \mathbb{C}^{\times}
$$

Let us take a number $q \in \mathbb{C}^{\times}$and consider the delta-functional $\delta^{q}$ in the point $q$ :

$$
\delta^{q}(u)=u(q)=\sum_{n \in \mathbb{Z}} u_{n} \cdot q^{n}, \quad u \in \mathcal{R}\left(\mathbb{C}^{\times}\right)
$$

Certainly, this is a current on $\mathbb{C}^{\times}$, i.e. an element of the space $\mathcal{R}^{\star}\left(\mathbb{C}^{\times}\right)$. Its expansion in basis $\zeta_{n}$ has the form:

$$
\delta^{q}=\sum_{n \in \mathbb{Z}} q^{n} \cdot \zeta_{n}
$$

Proposition 7.1. Elements $\left(z, \delta^{q}\right)$ form a quantum pair in the rigid stereotype Hopf algebras $\mathcal{R}\left(\mathbb{C}^{\times}\right)$and $\mathcal{O}\left(\mathbb{C}^{\times}\right)$with the parameter $q$. 
Proof. Let us prove this for $\mathcal{R}\left(\mathbb{C}^{\times}\right)$. We need to verify that $z$ and $\delta^{q}$ are central and group-like elements. The first property is trivial, since $\mathcal{R}\left(\mathbb{C}^{\times}\right)$and $\mathcal{R}^{\star}\left(\mathbb{C}^{\times}\right)$are commutative algebras. The fact that $z$ is a group-like element follows from (3.37):

$$
\varkappa(z)=z \odot z
$$

And for $\delta^{q}$ this follows from multiplicativity of delta-functionals:

$$
\begin{aligned}
\left\langle u \odot v, \varkappa\left(\delta^{q}\right)\right\rangle=\left\langle u \cdot v, \delta^{q}\right\rangle=(u \cdot v)(q)=u(q) \cdot v(q)=\left\langle u, \delta^{q}\right\rangle \cdot\left\langle v, \delta^{q}\right\rangle=\left\langle u \odot v, \delta^{q} \circledast \delta^{q}\right\rangle & \Longrightarrow \quad \varkappa\left(\delta^{q}\right)=\delta^{q} \circledast \delta^{q} .
\end{aligned}
$$

Finally the equalities (7.12) are verified directly:

$$
\left\langle\mathrm{M}_{\delta^{q}}^{\star} z, \alpha\right\rangle=\left\langle z, \delta^{q} * \alpha\right\rangle=\left\langle z, \sum_{m \in \mathbb{Z}} q^{m} \zeta_{m} * \sum_{n \in \mathbb{Z}} \alpha_{n} \zeta_{n}\right\rangle=(\underline{3.33})=\left\langle z, \sum_{n \in \mathbb{Z}} q^{n} \cdot \alpha_{n} \cdot \zeta_{n}\right\rangle=q \cdot \alpha_{1}=q \cdot\langle z, \alpha\rangle
$$

and

$$
\begin{aligned}
\left\langle u, \mathrm{M}_{z}^{\star} \delta^{q}\right\rangle=\left\langle z \cdot u, \delta^{q}\right\rangle=\left\langle\sum_{m \in \mathbb{Z}} u_{m} \cdot z^{m+1}, \sum_{n \in \mathbb{Z}} q^{n} \cdot \zeta_{n}\right\rangle & = \\
= & \left\langle\sum_{m \in \mathbb{Z}} u_{m} \cdot z^{m+1}, \sum_{l \in \mathbb{Z}} q^{l+1} \cdot \zeta_{l+1}\right\rangle=\sum_{m \in \mathbb{Z}} u_{m} \cdot q^{m+1}=q \cdot \sum_{m \in \mathbb{Z}} u_{m} \cdot q^{m}=q \cdot\left\langle u, \delta^{q}\right\rangle
\end{aligned}
$$

Hopf algebras $H \odot_{\omega}^{z} \mathcal{R}(\mathbb{C})$ and $H^{\star} \circledast_{z}^{\omega} \mathcal{R}^{\star}(\mathbb{C})$. In the following theorem $\theta$ means the isomorphism of functors (1.13), and $(k) !_{q}$ the quantum factorial from (7.2):

Theorem 7.6. Let $H$ be an injective stereotype Hopf algebra and let $(z, \omega)$ be a quantum pair in $H$ with the parameter $q \in \mathbb{C}^{\times}$. Then

(a) the tensor product $H \odot \mathcal{R}(\mathbb{C})$ has a unique structure of injective Hopf algebra with the algebraic operations defined by formulas:

$$
\begin{array}{rr}
\text { multiplication: } & a \odot t^{k} \cdot b \odot t^{l}=a \cdot\left(\mathrm{M}_{\omega}^{\star}\right)^{k}(b) \odot t^{k+l} \\
\text { unit: } & 1_{H \odot \mathcal{R}(\mathbb{C})}=1_{H} \odot 1_{\mathcal{R}(\mathbb{C})} \\
\text { comultiplication: } & \varkappa\left(a \odot t^{k}\right)=\sum_{i=0}^{k}\left(\begin{array}{c}
k \\
i
\end{array}\right)_{q} \cdot \theta\left(\left(1_{H} \odot \mathrm{M}_{z}^{i}\right)\left(\varkappa_{H}(a)\right) \odot t^{i} \odot t^{k-i}\right)= \\
& =\sum_{(a)} \sum_{i=0}^{k}\left(\begin{array}{c}
k \\
i
\end{array}\right)_{q} \cdot a^{\prime} \odot t^{i} \odot\left(z^{i} \cdot a^{\prime \prime}\right) \odot t^{k-i} \\
\text { counit: } & \varepsilon\left(a \odot t^{k}\right)= \begin{cases}\varepsilon_{H}(a), & k=0 \\
0, & k>0\end{cases} \\
\text { antipode: } & \sigma\left(a \odot t^{k}\right)=(-1)^{k} \cdot q^{-\frac{k(k+1)}{2}} \cdot z^{-k} \cdot\left(\mathrm{M}_{\omega}^{\star}\right)^{k}\left(\sigma_{H}(a)\right) \odot t^{k}
\end{array}
$$

$H \odot \mathcal{R}(\mathbb{C})$ with such a structure of Hopf algebra is denoted by $H \underset{\omega}{\stackrel{z}{*} \mathcal{R}}(\mathbb{C}) ;$ the common formula for multiplication in this algebra has the form:

$$
u \cdot v=\left(\sum_{k \in \mathbb{N}} u_{k} \odot t^{k}\right) \cdot\left(\sum_{l \in \mathbb{N}} v_{l} \odot t^{l}\right)=\sum_{m \in \mathbb{N}}\left(\sum_{k=0}^{m} u_{k} \cdot\left(\mathrm{M}_{\omega}^{\star}\right)^{k}\left(v_{m-k}\right)\right) \odot t^{m}
$$


(b) the tensor product $H^{\star} \circledast \mathcal{R}^{\star}(\mathbb{C})$ has a unique structure of projective Hopf algebra with the algebraic operations defined by formulas

$$
\begin{array}{rr}
\text { multiplication: } & \alpha \circledast \tau^{k} * \beta \circledast \tau^{l}=\alpha \cdot\left(\mathrm{M}_{z}^{\star}\right)^{k}(\beta) \circledast \tau^{k+l} \\
\text { unit: } & 1_{H^{\star} \circledast \mathcal{R}(\mathbb{C})}=1_{H^{\star}} \circledast 1_{\mathcal{R}^{\star}(\mathbb{C})} \\
\text { comultiplication: } & \varkappa\left(\alpha \circledast \tau^{k}\right)=\sum_{i=0}^{k}\left(\begin{array}{c}
k \\
i
\end{array}\right)_{q} \cdot \theta\left(\left(\operatorname{id}_{H^{\star}} \circledast \mathrm{M}_{\omega}^{i}\right)\left(\varkappa_{H^{\star}}(\alpha)\right) \circledast \tau^{i} \circledast \tau^{k-i}\right)= \\
=\sum_{(\alpha)} \sum_{i=0}^{k}\left(\begin{array}{c}
k \\
i
\end{array}\right)_{q} \cdot \alpha^{\prime} \circledast \tau^{i} \circledast\left(\omega^{i} * \alpha^{\prime \prime}\right) \circledast \tau^{k-i} \\
\text { counit: } \quad \varepsilon\left(\alpha \circledast \tau^{k}\right)= \begin{cases}\varepsilon_{H^{\star}}(\alpha), & k=0 \\
0, & k>0\end{cases} \\
\text { antipode: } & \sigma\left(\alpha \circledast \tau^{k}\right)=(-1)^{k} \cdot q^{-\frac{k(k+1)}{2}} \cdot \omega^{-k} *\left(\mathrm{M}_{z}^{\star}\right)^{k}\left(\sigma_{H^{\star}}(\alpha)\right) \circledast \tau^{k}
\end{array}
$$

$H^{\star} \circledast \mathcal{R}^{\star}(\mathbb{C})$ with such a structure of Hopf algebra is denoted by $H^{\star} \stackrel{\omega}{\circledast} \mathcal{R}^{\star}(\mathbb{C})$; the common formula for multiplication in this algebra has the form:

$$
\alpha * \beta=\left(\sum_{k \in \mathbb{N}} \alpha_{k} \odot \tau^{k}\right) \cdot\left(\sum_{l \in \mathbb{N}} \beta_{l} \odot \tau^{l}\right)=\sum_{m \in \mathbb{N}}\left(\sum_{k=0}^{m} \alpha_{k} *\left(\mathbf{M}_{z}^{\star}\right)^{k}\left(\beta_{m-k}\right)\right) \odot \tau^{m}
$$

(c) the bilinear form

$$
\left\langle\sum_{k \in \mathbb{N}} u_{k} \odot t^{k}, \sum_{k \in \mathbb{N}} \alpha_{k} \circledast \tau^{k}\right\rangle=\sum_{k \in \mathbb{N}}\left\langle u_{k}, \alpha_{k}\right\rangle \cdot(k) !_{q}
$$

turns $H \underset{\omega}{\stackrel{乛}{\odot}} \mathcal{R}(\mathbb{C})$ and $H^{\star} \underset{z}{\stackrel{\omega}{\circledast}} \mathcal{R}^{\star}(\mathbb{C})$ into a dual pair of stereotype Hopf algebras:

$$
(H \underset{\omega}{\stackrel{\sim}{\odot} \mathcal{R}} \mathcal{R}(\mathbb{C}))^{\star} \cong H^{\star} \underset{z}{\stackrel{\omega}{\circledast}} \mathcal{R}^{\star}(\mathbb{C})
$$

We divide the proof of this theorem in 7 lemmas. Some of them are evident, and in those cases we omit the proof.

Lemma 7.1. The multiplication and the unit (7.14), (7.15) endow $H \odot \mathcal{R}(\mathbb{C})$ with the structure of injective stereotype algebra, isomorphic to the algebra of skew polynomials with coefficients in algebra $H$ and the generating automorphism

$$
\varphi=\mathrm{M}_{\omega}^{\star}
$$

Lemma 7.2. The multiplication and the unit (7.20), (7.21) endow $H^{\star} \circledast \mathcal{R}^{\star}(\mathbb{C})$ with the structure of projective stereotype algebra, isomorphic to the algebra of skew power series with coefficients in algebra $H^{\star}$ and the generating automorphism

$$
\varphi=\mathrm{M}_{z}^{\star}
$$

Lemma 7.3. The bilinear form (7.26) turns the comultiplication (7.16) into the multiplication (7.20), and the counit (7.17) into the counit (7.21):

$$
\langle\varkappa(u), \alpha \circledast \beta\rangle=\langle u, \alpha * \beta\rangle, \quad \varepsilon(u)=\left\langle u, 1_{H^{\star}} \circledast 1_{\mathcal{R}^{\star}(\mathbb{C})}\right\rangle
$$

As a corollary, the comultiplication (7.16) and the counit (7.17) define the structure of injective stereotype coalgebra on $H \odot \mathcal{R}(\mathbb{C})$. 
Lemma 7.4. The bilinear form (7.26) turns multiplication (7.14) into comultiplication (7.22), and unit (7.15) into counit (7.23):

$$
\langle u \cdot v, \alpha\rangle=\langle u \odot v, \varkappa(\alpha)\rangle, \quad\left\langle 1_{H} \odot 1_{\mathcal{R}(\mathbb{C})}, \alpha\right\rangle=\varepsilon(\alpha)
$$

As a corollary, comultiplication (7.22) and counit (7.23) define the structure of projective stereotype coalgebra on $H^{\star} \circledast \mathcal{R}^{\star}(\mathbb{C})$.

Proof of Lemmas 7.3 and 7.4. Because of the symmetry between formulas (7.16)-(7.20) and (7.14)-(7.22) it is sufficient to prove (7.28). For this we can take $u=a \odot t^{k}$. Then the second equality becomes evident

$$
\varepsilon\left(a \odot t^{k}\right)=\left\{\begin{array}{cc}
\varepsilon_{H}(a), & k=0 \\
0, & k>0
\end{array}\right\}=\left\langle a \odot t^{k}, 1_{H^{\star}} \circledast 1_{\mathcal{R}^{\star}(\mathbb{C})}\right\rangle
$$

and the first one is proved by the following chain:

$$
\begin{gathered}
\left\langle\varkappa\left(a \odot t^{k}\right), \alpha \circledast \beta\right\rangle=\sum_{i=0}^{k}\left(\begin{array}{c}
k \\
i
\end{array}\right)_{q} \cdot\left\langle\theta\left(\left(1_{H} \odot \mathrm{M}_{z}^{i}\right)\left(\varkappa_{H}(a)\right) \odot t^{i} \odot t^{k-i}\right), \sum_{l \in \mathbb{N}} \alpha_{l} \circledast \tau^{l} \circledast \sum_{m \in \mathbb{N}} \beta_{m} \circledast \tau^{m}\right\rangle= \\
=\sum_{l, m \in \mathbb{N}} \sum_{i=0}^{k}\left(\begin{array}{c}
k \\
i
\end{array}\right)_{q} \cdot\left\langle\theta\left(\left(1_{H} \odot \mathrm{M}_{z}^{i}\right)\left(\varkappa_{H}(a)\right) \odot t^{i} \odot t^{k-i}\right), \alpha_{l} \circledast \tau^{l} \circledast \beta_{m} \circledast \tau^{m}\right\rangle= \\
=\sum_{l, m \in \mathbb{N}} \sum_{i=0}^{k}\left(\begin{array}{c}
k \\
i
\end{array}\right)_{q} \cdot\left\langle\left(1_{H} \odot \mathrm{M}_{z}^{i}\right)\left(\varkappa_{H}(a)\right) \odot t^{i} \odot t^{k-i}, \theta\left(\alpha_{l} \circledast \tau^{l} \circledast \beta_{m} \circledast \tau^{m}\right)\right\rangle= \\
=\sum_{l, m \in \mathbb{N}} \sum_{i=0}^{k}\left(\begin{array}{c}
k \\
i
\end{array}\right)_{q} \cdot\left\langle\left(1_{H} \odot \mathrm{M}_{z}^{i}\right)\left(\varkappa_{H}(a)\right) \odot t^{i} \odot t^{k-i}, \alpha_{l} \circledast \beta_{m} \circledast \tau^{l} \circledast \tau^{m}\right\rangle= \\
=\sum_{i=0}^{k}\left(\begin{array}{c}
k \\
i
\end{array}\right)_{q} \cdot(i) !_{q} \cdot(k-i) !_{q}\left\langle\left(1_{H} \odot \mathrm{M}_{z}^{i}\right)\left(\varkappa_{H}(a)\right), \alpha_{i} \circledast \beta_{k-i}\right\rangle= \\
=(k) !_{q} \cdot \sum_{i=0}^{k}\left\langle\varkappa_{H}(a), \alpha_{i} \circledast\left(\mathrm{M}_{z}^{\star}\right)^{i}\left(\beta_{k-i}\right)\right\rangle=(k) !_{q} \cdot \sum_{i=0}^{k}\left\langle a, \alpha_{i} *\left(\mathrm{M}_{z}^{\star}\right)^{i}\left(\beta_{k-i}\right)\right\rangle= \\
=\left\langle a \odot t^{k}, \sum_{i=0}^{k} \alpha_{i} *\left(\mathrm{M}_{z}^{\star}\right)^{i}\left(\beta_{k-i} \circledast \tau^{k}\right\rangle=\left\langle a \odot t^{k}, \sum_{n \in \mathbb{N}}\left(\sum_{i=0}^{n} \alpha_{i} *\left(\mathrm{M}_{z}^{\star}\right)^{i}\left(\beta_{n-i}\right)\right) \circledast \tau^{n}\right\rangle=\right. \\
=\left\langle a \odot t^{k}, \alpha * \beta\right\rangle
\end{gathered}
$$

Lemma 7.5. Comultiplication (7.16) and counit (7.17) are homomorphisms of injective stereotype algebras, and as a corollary endow $H \odot \mathcal{R}(\mathbb{C})$ with the structure of injective stereotype bialgebra.

Lemma 7.6. Comultiplication (7.22) and counit (7.23) are homomorphisms of projective stereotype algebras, and as a corollary endow $H^{\star} \circledast \mathcal{R}^{\star}(\mathbb{C})$ with the structure of projective stereotype bialgebra.

Proof. Again due to the symmetry of formulas it is sufficient here to prove the first lemma. We will just check that the comultiplication (7.16) is a homomorphism of algebras (and the reader is supposed to check the identity for counit by analogy). Let us note the following identities:

$$
\begin{aligned}
\varkappa(a \odot 1 \cdot b \odot 1) & =\varkappa(a \odot 1) \cdot \varkappa(b \odot 1) \\
\varkappa\left(1 \odot t^{k} \cdot 1 \odot t^{l}\right) & =\varkappa\left(1 \odot t^{k}\right) \cdot \varkappa\left(1 \odot t^{l}\right) \\
\varkappa\left(1 \odot t^{k} \cdot a \odot 1\right) & =\varkappa\left(1 \odot t^{k}\right) \cdot \varkappa(a \odot 1)
\end{aligned}
$$




$$
\varkappa\left(a \odot 1 \cdot 1 \odot t^{k}\right)=\varkappa(a \odot 1) \cdot \varkappa\left(1 \odot t^{k}\right)
$$

Indeed, for (7.30) we have:

$$
\begin{aligned}
\varkappa(a \odot 1) \cdot \varkappa(b \odot 1)=\sum_{(a)}\left(a^{\prime} \odot 1 \odot a^{\prime \prime} \odot 1\right) \cdot \sum_{(b)}\left(b^{\prime} \odot 1 \odot b^{\prime \prime} \odot 1\right)= \\
=\sum_{(a),(b)} a^{\prime} b^{\prime} \odot 1 \odot a^{\prime \prime} b^{\prime \prime} \odot 1=\varkappa((a \cdot b) \odot 1)=\varkappa(a \odot 1 \cdot b \odot 1)
\end{aligned}
$$

For (7.31):

$$
\begin{aligned}
& \varkappa\left(1 \odot t^{k}\right) \cdot \varkappa\left(1 \odot t^{k}\right)=\sum_{i=0}^{k}\left(\begin{array}{l}
k \\
i
\end{array}\right)_{q} \cdot 1 \odot t^{i} \odot z^{i} \odot t^{k-i} \cdot \sum_{j=0}^{l}\left(\begin{array}{l}
l \\
j
\end{array}\right)_{q} \cdot 1 \odot t^{j} \odot z^{j} \odot t^{l-j}= \\
& =\sum_{i=0}^{k} \sum_{j=0}^{l}\left(\begin{array}{l}
k \\
i
\end{array}\right)_{q} \cdot\left(\begin{array}{l}
l \\
j
\end{array}\right)_{q} \cdot\left(\left(1 \odot t^{i}\right) \cdot\left(1 \odot t^{j}\right)\right) \odot\left(\left(z^{i} \odot t^{k-i}\right) \cdot\left(z^{j} \odot t^{l-j}\right)\right)=(\underline{7.14})= \\
& =\sum_{i=0}^{k} \sum_{j=0}^{l}\left(\begin{array}{l}
k \\
i
\end{array}\right)_{q} \cdot\left(\begin{array}{l}
l \\
j
\end{array}\right)_{q} \cdot\left(1 \odot t^{i+j}\right) \odot\left(\left(z^{i} \cdot\left(\mathrm{M}_{\omega}^{\star}\right)^{k-i}\left(z^{j}\right) \odot t^{k+l-i-j}\right)=(\mathbf{7 . 1 2})=\right. \\
& =\sum_{i=0}^{k} \sum_{j=0}^{l}\left(\begin{array}{l}
k \\
i
\end{array}\right)_{q} \cdot\left(\begin{array}{l}
l \\
j
\end{array}\right)_{q} \cdot q^{(k-i) j} \cdot 1 \odot t^{i+j} \odot z^{i+j} \odot t^{k+l-i-j}= \\
& =\left(\begin{array}{c}
i+j=m \\
j=m-i \\
0 \leqslant m \leqslant k+l \\
\max \{0, m-l\} \leqslant i \leqslant \min \{k, m\}
\end{array}\right)= \\
& =\sum_{m=0}^{k+l} \sum_{\max \{0, m-l\} \leqslant i \leqslant \min \{k, m\}}\left(\begin{array}{c}
k \\
i
\end{array}\right)_{q} \cdot\left(\begin{array}{c}
l \\
m-i
\end{array}\right)_{q} \cdot q^{(k-i)(m-i)} \cdot 1 \odot t^{m} \odot z^{m} \odot t^{k+l-m}=(7.5)= \\
& =\sum_{m=0}^{k+l}\left(\begin{array}{c}
k+l \\
m
\end{array}\right)_{q} \cdot 1 \odot t^{m} \odot z^{m} \odot t^{k+l-m}=\varkappa\left(1 \odot t^{k+l}\right)=\varkappa\left(1 \odot t^{k} \cdot 1 \odot t^{l}\right)
\end{aligned}
$$

For (7.32):

$$
\begin{aligned}
& \varkappa\left(1 \odot t^{k}\right) \cdot \varkappa(a \odot 1)=\sum_{i=0}^{k}\left(\begin{array}{l}
k \\
i
\end{array}\right)_{q} \cdot 1 \odot t^{i} \odot z^{i} \odot t^{k-i} \cdot \sum_{(a)} a^{\prime} \odot 1 \odot a^{\prime \prime} \odot 1= \\
& =\sum_{i=0}^{k} \sum_{(a)}\left(\begin{array}{l}
k \\
i
\end{array}\right)_{q} \cdot\left(\left(1 \odot t^{i}\right) \cdot\left(a^{\prime} \odot 1\right)\right) \odot\left(\left(z^{i} \odot t^{k-i}\right) \cdot\left(a^{\prime \prime} \odot 1\right)\right)= \\
& \left.=\sum_{i=0}^{k} \sum_{(a)}\left(\begin{array}{c}
k \\
i
\end{array}\right)_{q} \cdot\left(\mathrm{M}_{\omega}^{\star}\right)^{i}\left(a^{\prime}\right) \odot t^{i} \odot z^{i}\left(\mathrm{M}_{\omega}^{\star}\right)^{k-i}\left(a^{\prime \prime}\right) \odot t^{k-i}=\underline{1.40}\right)= \\
& =\varkappa\left(\left(\mathrm{M}_{\omega}^{\star}\right)^{k}(a) \odot t^{k}\right)=(\underline{7.14})=\varkappa\left(1 \odot t^{k} \cdot a \odot 1\right)
\end{aligned}
$$

For (7.33):

$$
\begin{aligned}
\varkappa(a \odot 1) \cdot \varkappa\left(1 \odot t^{k}\right) & =\sum_{(a)} a^{\prime} \odot 1 \odot a^{\prime \prime} \odot 1 \cdot \sum_{i=0}^{k}\left(\begin{array}{c}
k \\
i
\end{array}\right)_{q} \cdot 1 \odot t^{i} \odot z^{i} \odot t^{k-i}= \\
= & \sum_{(a)} \sum_{i=0}^{k}\left(\begin{array}{c}
k \\
i
\end{array}\right)_{q} \cdot\left(\left(a^{\prime} \odot 1\right) \cdot\left(1 \odot t^{i}\right)\right) \odot\left(\left(a^{\prime \prime} \odot 1\right) \cdot\left(z^{i} \odot t^{k-i}\right)\right)=
\end{aligned}
$$




$$
=\sum_{(a)} \sum_{i=0}^{k}\left(\begin{array}{c}
k \\
i
\end{array}\right)_{q} \cdot a^{\prime} \odot t^{i} \odot a^{\prime \prime} z^{i} \odot t^{k-i}=(\underline{7.16})=\varkappa\left(a \odot t^{k}\right)=\varkappa\left(a \odot 1 \cdot 1 \odot t^{k}\right)
$$

From (7.30)-(7.33) it follows that (7.16) is a homomorphism a algebras:

$$
\begin{aligned}
& \varkappa\left(a \odot t^{k} \cdot b \odot t^{l}\right)=\varkappa\left(a \cdot\left(\mathrm{M}_{\omega}^{\star}\right)^{k}(b) \odot t^{k+l}\right)=\varkappa\left(a \cdot\left(\mathrm{M}_{\omega}^{\star}\right)^{k}(b) \odot 1 \cdot 1 \odot t^{k+l}\right)=(7.33)= \\
& =\varkappa\left(a \cdot\left(\mathrm{M}_{\omega}^{\star}\right)^{k}(b) \odot 1\right) \cdot \varkappa\left(1 \odot t^{k+l}\right)=\varkappa\left(a \odot 1 \cdot\left(\mathrm{M}_{\omega}^{\star}\right)^{k}(b) \odot 1\right) \cdot \varkappa\left(1 \odot t^{k} \cdot 1 \odot t^{l}\right)= \\
& =\left(\text { (7.30) },(17.31)=\varkappa(a \odot 1) \cdot \varkappa\left(\left(\mathrm{M}_{\omega}^{\star}\right)^{k}(b) \odot 1\right) \cdot \varkappa\left(1 \odot t^{k}\right) \cdot \varkappa\left(1 \odot t^{l}\right)=\right. \\
& =\underline{(7.33)}=\varkappa(a \odot 1) \cdot \varkappa\left(\left(\mathrm{M}_{\omega}^{\star}\right)^{k}(b) \odot 1 \cdot 1 \odot t^{k}\right) \cdot \varkappa\left(1 \odot t^{l}\right)= \\
& =\varkappa(a \odot 1) \cdot \varkappa\left(1 \odot t^{k} \cdot b \odot 1\right) \cdot \varkappa\left(1 \odot t^{l}\right)= \\
& =(\underline{7.32})=\varkappa(a \odot 1) \cdot \varkappa\left(1 \odot t^{k}\right) \cdot \varkappa(b \odot 1) \cdot \varkappa\left(1 \odot t^{l}\right)=(\underline{7.33})= \\
& =\varkappa\left(a \odot 1 \cdot 1 \odot t^{k}\right) \cdot \varkappa\left(b \odot 1 \cdot 1 \odot t^{l}\right)=\varkappa\left(a \odot t^{k}\right) \cdot \varkappa\left(b \odot t^{l}\right)
\end{aligned}
$$

Lemma 7.7. Formulas (7.18) and (7.24) define antipodes in bialgebras $H \odot \mathcal{R}(\mathbb{C})$ and $H^{\star} \circledast \mathcal{R} \star(\mathbb{C})$, dual to each other with respect to the bilinear form (7.26):

$$
\langle\sigma(u), \alpha\rangle=\langle u, \sigma(\alpha)\rangle
$$

Proof. Let us show that formula (7.18) defines an antipode in $H \odot \mathcal{R}(\mathbb{C})$. First we need to verify that $\sigma$ is an automorphism:

$$
\begin{gathered}
\sigma\left(a \odot t^{k} \cdot b \odot t^{l}\right)=\sigma\left(a \cdot\left(\mathrm{M}_{\omega}^{\star}\right)^{k}(b) \odot t^{k+l}\right)= \\
=(-1)^{k+l} \cdot q^{-\frac{(k+l)(k+l+1)}{2}} \cdot z^{-k-l} \cdot\left(\mathrm{M}_{\omega}^{\star}\right)^{k+l}\left(\sigma_{H}\left(a \cdot\left(\mathrm{M}_{\omega}^{\star}\right)^{k}(b)\right)\right) \odot t^{k+l}= \\
=(-1)^{k+l} \cdot q^{-\frac{(k+l)(k+l+1)}{2}} \cdot z^{-k-l} \cdot\left(\mathrm{M}_{\omega}^{\star}\right)^{k+l}\left(\sigma_{H}\left(\left(\mathrm{M}_{\omega}^{\star}\right)^{k}(b)\right) \cdot \sigma_{H}(a)\right) \odot t^{k+l}= \\
=(-1)^{k+l} \cdot q^{-\frac{(k+l)(k+l+1)}{2}} \cdot z^{-k-l} \cdot\left(\mathrm{M}_{\omega}^{\star}\right)^{l}\left(\left(\left(\mathrm{M}_{\omega}^{\star}\right)^{k} \circ \sigma_{H} \circ\left(\mathrm{M}_{\omega}^{\star}\right)^{k}\right)(b)\right) \cdot\left(\mathrm{M}_{\omega}^{\star}\right)^{k+l}\left(\sigma_{H}(a)\right) \odot t^{k+l}= \\
=\underline{(1.38)}=(-1)^{k+l} \cdot q^{-\frac{k^{2}+l^{2}+k+l}{2}} \cdot q^{-k l} \cdot z^{-k-l} \cdot\left(\mathrm{M}_{\omega}^{\star}\right)^{l}\left(\sigma_{H}(b)\right) \cdot\left(\mathrm{M}_{\omega}^{\star}\right)^{k+l}\left(\sigma_{H}(a)\right) \odot t^{k+l}= \\
=(-1)^{k+l} \cdot q^{-\frac{k(k+1)+l(l+1)}{2}} \cdot z^{-l} \cdot\left(\mathrm{M}_{\omega}^{\star}\right)^{l}\left(\sigma_{H}(b)\right) \cdot\left(\mathrm{M}_{\omega}^{\star}\right)^{l}\left(z^{-k}\left(\mathrm{M}_{\omega}^{\star}\right)^{k}\left(\sigma_{H}(a)\right)\right) \odot t^{k+l}= \\
=(-1)^{l} \cdot q^{-\frac{l(l+1)}{2}} \cdot z^{-l} \cdot\left(\mathrm{M}_{\omega}^{\star}\right)^{l}\left(\sigma_{H}(b)\right) \odot t^{l} \cdot(-1)^{k} \cdot q^{-\frac{k(k+1)}{2}} \cdot z^{-k} \cdot\left(\mathrm{M}_{\omega}^{\star}\right)^{k}\left(\sigma_{H}(a)\right) \odot t^{k}= \\
=\sigma\left(b \odot t^{l}\right) \cdot \sigma\left(a \odot t^{k}\right)
\end{gathered}
$$


Now let us note that diagram (1.18) becomes commutative if we put there $a \odot 1$ :

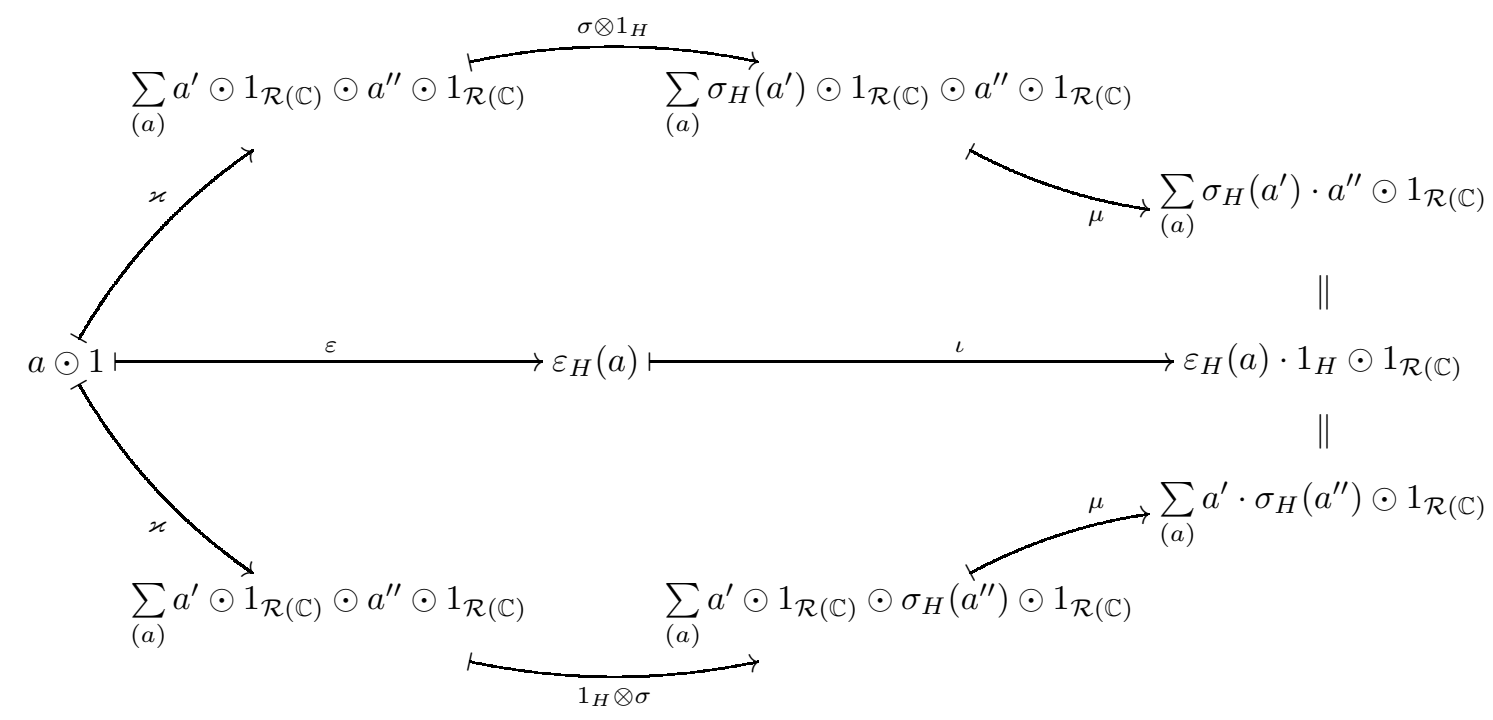

or $1 \odot t$ :

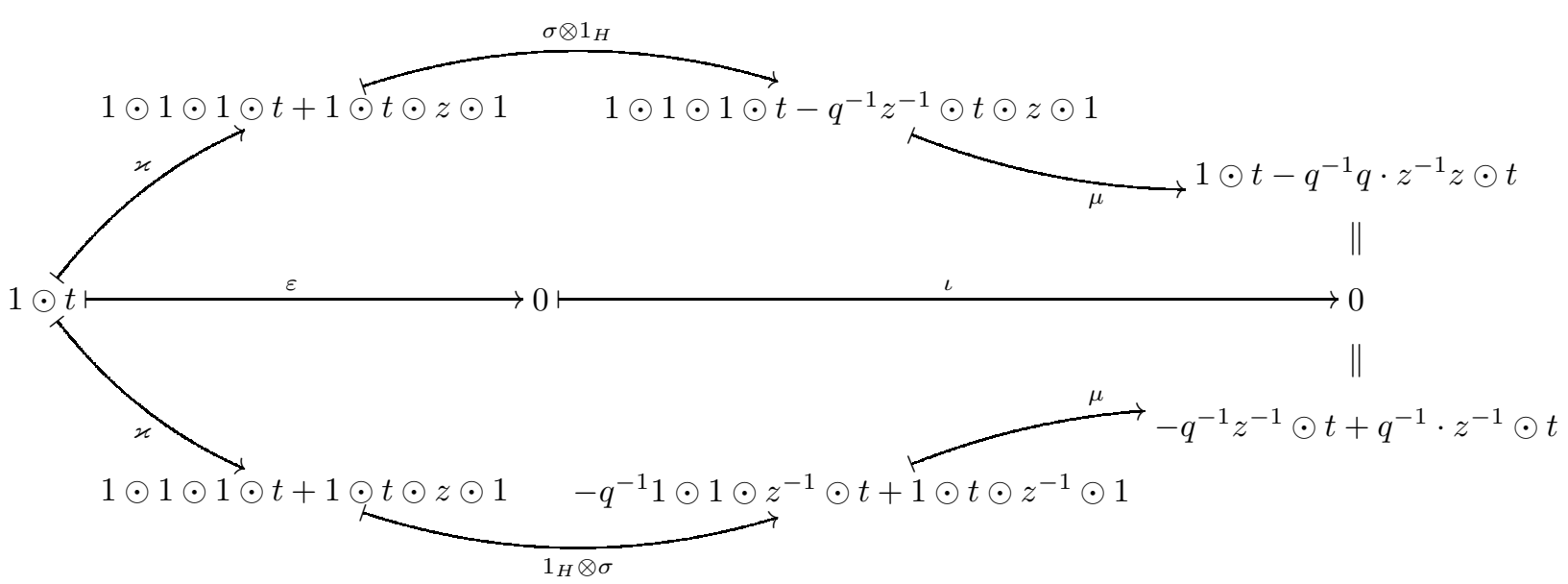

By Lemma on antipode 1.1 this implies that the diagram (1.18) is commutative if we put there after various products of $a \odot 1$ and $1 \odot t$. In particular, if we put $a \odot t^{k}$. This means that 1.1 is commutative (with arbitrary argument), and we obtain that the mapping (7.18) is an antipode in $H \odot \mathcal{R}(\mathbb{C})$.

Further, by the symmetry of formulas, the mapping (7.24) is an antipode in $H^{\star} \circledast \mathcal{R}^{\star}(\mathbb{C})$.

It remains to verify that the bilinear form (7.26) turns the antipode (7.18) into the antipode (7.24). Clearly, formula (7.34) is equivalent to formula

$$
\left\langle\sigma\left(a \odot t^{k}\right), \alpha \circledast \tau^{l}\right\rangle=\left\langle a \odot t^{k}, \sigma\left(\alpha \circledast \tau^{l}\right)\right\rangle
$$

For $k \neq l$ both sides here vanish, so we need check only the case $k=l$. Indeed,

$$
\begin{aligned}
& \left\langle\sigma\left(a \odot t^{k}\right), \alpha \circledast \tau^{k}\right\rangle=(\mathbf{7 . 1 8})=\left\langle(-1)^{k} \cdot q^{-\frac{k(k+1)}{2}} \cdot z^{-k} \cdot\left(\mathrm{M}_{\omega}^{\star}\right)^{k}\left(\sigma_{H}(a)\right) \odot t^{k}, \alpha \circledast \tau^{k}\right\rangle= \\
= & (-1)^{k} \cdot q^{-\frac{k(k+1)}{2}} \cdot\left\langle z^{-k} \cdot\left(\mathrm{M}_{\omega}^{\star}\right)^{k}\left(\sigma_{H}(a)\right), \alpha\right\rangle \cdot(k) !_{q}=(-1)^{k} \cdot q^{-\frac{k(k+1)}{2}} \cdot\left\langle\left(\mathrm{M}_{\omega}^{\star}\right)^{k}\left(\sigma_{H}(a)\right),\left(\mathrm{M}_{z^{-1}}^{\star}\right)^{k}(\alpha)\right\rangle \cdot(k) !_{q}= \\
= & (-1)^{k} \cdot q^{-\frac{k(k+1)}{2}} \cdot\left\langle\sigma_{H}(a), \omega^{k} *\left(\mathrm{M}_{z^{-1}}^{\star}\right)^{k}(\alpha)\right\rangle \cdot(k) !_{q}=(-1)^{k} \cdot q^{-\frac{k(k+1)}{2}} \cdot\left\langle a, \sigma_{H}^{\star}\left(\left(\omega^{k} *\left(\mathrm{M}_{z^{-1}}^{\star}\right)^{k}(\alpha)\right)\right\rangle \cdot(k) !_{q}=\right.
\end{aligned}
$$




$$
\begin{gathered}
=(-1)^{k} \cdot q^{-\frac{k(k+1)}{2}} \cdot\left\langle a, \omega^{-k} * \sigma_{H}^{\star}\left(\left(\mathrm{M}_{z^{-1}}^{\star}\right)^{k}(\alpha)\right)\right\rangle \cdot(k) !_{q}=(1.38) \\
=(-1)^{k} \cdot q^{-\frac{k(k+1)}{2}} \cdot\left\langle a, \omega^{-k} *\left(\mathrm{M}_{z}^{\star}\right)^{k}\left(\sigma_{H}^{\star}(\alpha)\right)\right\rangle \cdot(k) !_{q}=(-1)^{k} \cdot q^{-\frac{k(k+1)}{2}} \cdot\left\langle a \odot t^{k}, \omega^{-k} *\left(\mathrm{M}_{z}^{\star}\right)^{k}\left(\sigma_{H}^{\star}(\alpha)\right) \circledast \tau^{k}\right\rangle= \\
=(\mathbf{7 . 2 4})=\left\langle a \odot t^{k}, \sigma\left(\alpha \circledast \tau^{k}\right)\right\rangle
\end{gathered}
$$

Chains $H \odot_{\omega}^{z} \mathcal{R}(\mathbb{C}) \subset H \odot_{\omega}^{z} \mathcal{O}(\mathbb{C}) \subset H \odot_{\omega}^{z} \mathcal{O}^{\star}(\mathbb{C}) \subset H \odot_{\omega}^{z} \mathcal{R}^{\star}(\mathbb{C})$

and $H \circledast_{\omega}^{z} \mathcal{R}(\mathbb{C}) \subset H \circledast_{\omega}^{z} \mathcal{O}(\mathbb{C}) \subset H \circledast_{\omega}^{z} \mathcal{O}^{\star}(\mathbb{C}) \subset H \circledast_{\omega}^{z} \mathcal{R}^{\star}(\mathbb{C})$.

The same formulas and reasonings as we used in the proof of Theorem [7.6, allow us to define, apart

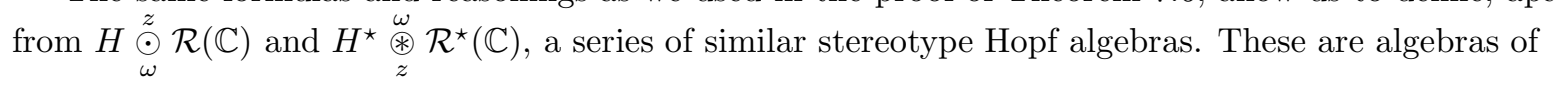

— skew polynomials $H \underset{\omega}{\stackrel{z}{\odot}} \mathcal{R}(\mathbb{C})$ and $H \underset{\omega}{\stackrel{z}{\circledast}} \mathcal{R}(\mathbb{C})$,

— skew entire functions $H \underset{\omega}{\stackrel{z}{\odot}} \mathcal{O}(\mathbb{C})$ and $H \underset{\omega}{\stackrel{z}{\circledast}} \mathcal{O}(\mathbb{C})$,

— skew analytic functionals $H \underset{\omega}{\stackrel{z}{\odot}} \mathcal{O}^{\star}(\mathbb{C})$ and $H \underset{\omega}{\stackrel{z}{\circledast}} \mathcal{O}^{\star}(\mathbb{C})$,

— skew power series $H \underset{\omega}{\stackrel{z}{\odot}} \mathcal{R}^{\star}(\mathbb{C})$ and $H \underset{\omega}{\stackrel{z}{\circledast}} \mathcal{R}^{\star}(\mathbb{C})$.

Visually the connection between them can be illustrated by the following chains of inclusions:

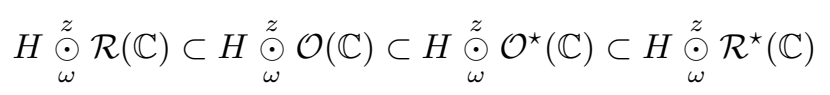

and

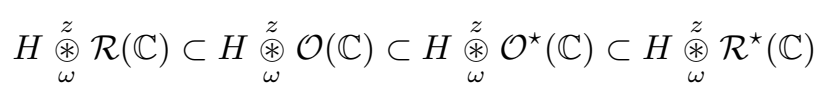

If $H$ is an injective Hopf algebra, then the upper chain is defined, if $H$ is a projective Hopf algebra, then the lower chain is defined, and if $H$ is a rigid stereotype Hopf algebra, then both chains are defined (and, certainly, they coincide up to isomorphisms).

Theorem 7.6 correctly define only the first link in the first chain and the last link in the second chain. In all conscience, to give accurate definition for all links we should formulate three more analogous theorems.

To avoid those troubles we can either simply say that the other links are defined by analogy (with replacing, if necessary, $\odot$ by $\circledast$, and $\mathcal{R}$ by $\mathcal{O}$ ). Or we can unite all those four theorems (the one already proven and three not yet formulated) into the following quite bulky proposition:

Theorem 7.7. Let

- $F$ denote one of the two Hopf algebras: $\mathcal{R}(\mathbb{C})$ or $\mathcal{O}(\mathbb{C})$,

- $H$ be an arbitrary injective stereotype Hopf algebra,

- $(z, \omega)$ be a quantum pair in $H$ with parameter $q \in \mathbb{C}^{\times}$.

Then $^{7}$

(a) the tensor product $H \odot F$ possesses a unique structure of injective Hopf algebra with algebraic operations, defined by formulas:

$$
\text { multiplication: } \quad a \odot t^{k} \cdot b \odot t^{l}=a \cdot\left(\mathrm{M}_{\omega}^{\star}\right)^{k}(b) \odot t^{k+l}
$$

\footnotetext{
${ }^{7}$ Here again $\theta$ is the isomorphism of functors (1.13), and $(k) !_{q}$ the quantum factorial defined in (7.2).
} 


$$
\begin{array}{rlrl}
\text { unit: } & 1_{H \odot \mathcal{R}(\mathbb{C})}=1_{H} \odot 1_{\mathcal{R}(\mathbb{C})} \\
\text { comultiplication: } & \varkappa\left(a \odot t^{k}\right)=\sum_{i=0}^{k}\left(\begin{array}{l}
k \\
i
\end{array}\right)_{q} \cdot \theta\left(\left(1_{H} \odot \mathrm{M}_{z}^{i}\right)\left(\varkappa_{H}(a)\right) \odot t^{i} \odot t^{k-i}\right)= \\
=\sum_{(a)} \sum_{i=0}^{k}\left(\begin{array}{c}
k \\
i
\end{array}\right)_{q} \cdot a^{\prime} \odot t^{i} \odot\left(z^{i} \cdot a^{\prime \prime}\right) \odot t^{k-i} \\
\text { counit: } & \varepsilon\left(a \odot t^{k}\right)= \begin{cases}\varepsilon_{H}(a), & k=0 \\
0, & k>0\end{cases} \\
\text { antipode: } & \sigma\left(a \odot t^{k}\right)=(-1)^{k} \cdot q^{-\frac{k(k+1)}{2}} \cdot z^{-k} \cdot\left(\mathrm{M}_{\omega}^{\star}\right)^{k}\left(\sigma_{H}(a)\right) \odot t^{k}
\end{array}
$$

$H \odot F$ with such a structure of Hopf algebra is denoted by $H \underset{\omega}{\stackrel{z}{\odot}} F$;

(b) the tensor product $H^{\star} \circledast F^{\star}$ possesses a unique structure of projective Hopf algebra with the algebraic operations, defined by formulas:

$$
\begin{aligned}
& \text { multiplication: } \alpha \circledast \tau^{k} * \beta \circledast \tau^{l}=\alpha \cdot\left(\mathrm{M}_{z}^{\star}\right)^{k}(\beta) \circledast \tau^{k+l} \\
& \text { unit: } 1_{H^{\star} \circledast \mathcal{R}(\mathbb{C})}=1_{H^{\star}} \circledast 1_{\mathcal{R}^{\star}(\mathbb{C})} \\
& \text { comultiplication: } \varkappa\left(\alpha \circledast \tau^{k}\right)=\sum_{i=0}^{k}\left(\begin{array}{c}
k \\
i
\end{array}\right)_{q} \cdot \theta\left(\left(\mathrm{id}_{H^{\star}} \circledast \mathrm{M}_{\omega}^{i}\right)\left(\varkappa_{H^{\star}}(\alpha)\right) \circledast \tau^{i} \circledast \tau^{k-i}\right)= \\
&=\sum_{(\alpha)} \sum_{i=0}^{k}\left(\begin{array}{l}
k \\
i
\end{array}\right)_{q} \cdot \alpha^{\prime} \circledast \tau^{i} \circledast\left(\omega^{i} \cdot \alpha^{\prime \prime}\right) \circledast \tau^{k-i} \\
& \text { counit: } \quad \varepsilon\left(\alpha \circledast \tau^{k}\right)= \begin{cases}\varepsilon_{H^{\star}}(\alpha), & k=0 \\
0, & k>0\end{cases} \\
& \text { antipode: } \quad \sigma\left(\alpha \circledast \tau^{k}\right)=(-1)^{k} \cdot q^{-\frac{k(k+1)}{2} \cdot \omega^{-k} *\left(\mathrm{M}_{z}^{\star}\right)^{k}\left(\sigma_{H^{\star}}(\alpha)\right) \circledast \tau^{k}}
\end{aligned}
$$

$H^{\star} \circledast F^{\star}$ with such a structure of Hopf algebra is denoted by $H^{\star} \underset{z}{\stackrel{\omega}{\circledast}} F^{\star}$;

(c) the bilinear form

$$
\left\langle\sum_{k \in \mathbb{N}} u_{k} \odot t^{k}, \sum_{k \in \mathbb{N}} \alpha_{k} \circledast \tau^{k}\right\rangle=\sum_{k \in \mathbb{N}}\left\langle u_{k}, \alpha_{k}\right\rangle \cdot(k) !_{q}
$$

turns $H \underset{\omega}{\stackrel{z}{\rho}} F$ and $H^{\star} \underset{z}{\stackrel{\omega}{\circledast}} F^{\star}$ into dual pair of stereotype Hopf algebras.

If, under all other assumptions (except injectivity), $H$ is a projective stereotype Hopf algebra, then

(a) the tensor product $H \circledast F$ has a unique structure of projective stereotype Hopf algebra with algebraic operations defined by formulas (7.35)-(7.39), but with replacing $\odot$ by $\circledast ; H \circledast F$ with such a structure of Hopf algebra is denoted by $H \underset{\omega}{\stackrel{z}{\circledast}} F$;

(b) the tensor product $H^{\star} \odot F^{\star}$ has a unique structure of injective stereotype Hopf algebra with algebraic operations defined by formulas (7.40)-(7.44), but with replacing $\circledast$ by $\odot ; H^{\star} \odot F^{\star}$ with such a structure of Hopf algebra is denoted by $H^{\star} \underset{z}{\stackrel{\omega}{\odot}} F^{\star}$

(c) the bilinear form

$$
\left\langle\sum_{k \in \mathbb{N}} u_{k} \circledast t^{k}, \sum_{k \in \mathbb{N}} \alpha_{k} \odot \tau^{k}\right\rangle=\sum_{k \in \mathbb{N}}\left\langle u_{k}, \alpha_{k}\right\rangle \cdot(k) !_{q}
$$


turns $H \underset{\omega}{\stackrel{z}{\circledast} F}$ and $H^{\star} \stackrel{\omega}{\odot} F^{\star}$ into a dual pair of stereotype Hopf algebras.

As we have already told this is proved by analogy with Theorem 7.6

Proposition 7.2. Let $H$ be an injective Hopf algebra, and $(z, \omega)$ a quantum pair in $H$ with the parameter $q \in \mathbb{C}^{\times}$. Then the rules

$$
a \odot t^{k} \mapsto a \odot t^{k} \mapsto a \odot \tau^{k} \mapsto a \odot \tau^{k} \quad(k \in \mathbb{N}, \quad a \in H)
$$

uniquely define a chain of (continuous) homomorphisms of injective stereotype Hopf algebras:

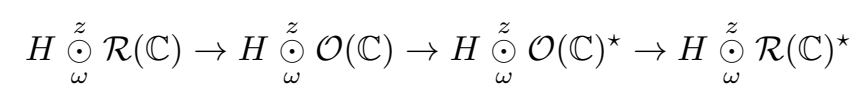

Proposition 7.3. Let $H$ be a projective Hopf algebra and $(z, \omega)$ a quantum pair in $H$ with the parameter $q \in \mathbb{C}^{\times}$. Then the rules

$$
a \circledast t^{k} \mapsto a \circledast t^{k} \mapsto a \circledast \tau^{k} \mapsto a \circledast \tau^{k} \quad(k \in \mathbb{N}, \quad a \in H)
$$

uniquely define a chain of (continuous) homomorphisms of projective stereotype Hopf algebras:

$$
H \underset{\omega}{\stackrel{z}{\circledast}} \mathcal{R}(\mathbb{C}) \rightarrow H \underset{\omega}{\stackrel{z}{\circledast}} \mathcal{O}(\mathbb{C}) \rightarrow H \underset{\omega}{\stackrel{z}{\circledast}} \mathcal{O}(\mathbb{C})^{\star} \rightarrow H \underset{\omega}{\stackrel{z}{\circledast}} \mathcal{R}(\mathbb{C})^{\star}
$$

\section{(c) Quantum group ' $a z+b^{\prime}=\mathcal{R}_{q}\left(\mathbb{C}^{\times} \ltimes \mathbb{C}\right)$}

Here we show that the quantum group ' $a z+b$ ' (defined in [48, 40, 47, 28]) is a special case of the construction described in Theorem 7.6 .

The group $\mathbb{C}^{\times} \ltimes \mathbb{C}$ of affine transformation of a complex plane. The group of affine transformations of the complex plane, often denoted as ' $a z+b$ ', from the algebraic point of view is a semidirect product $\mathbb{C}^{\times} \ltimes \mathbb{C}$ of complex circle $\mathbb{C}^{\times}$and complex plane $\mathbb{C}$, where $\mathbb{C}^{\times}$acts on $\mathbb{C}$ by usual multiplication. In other word, $\mathbb{C}^{\times} \ltimes \mathbb{C}$ is a Cartesian product $\mathbb{C}^{\times} \times \mathbb{C}$ with algebraic operations

$$
\begin{aligned}
& \text { multiplication: } \\
& (a, x) \cdot(b, y)=(a b, x b+y) \quad\left(a, b \in \mathbb{C}^{\times}, x, y \in \mathbb{C}\right) \\
& \text { unit: } \\
& 1_{\mathbb{C}^{\times} \ltimes \mathbb{C}}=(1,0) \\
& \text { inverse element: } \\
& (a, x)^{-1}=\left(\frac{1}{a},-\frac{x}{a}\right) \\
& \left(a \in \mathbb{C}^{\times}, x \in \mathbb{C}\right)
\end{aligned}
$$

Clearly, this is a connected Stein group. Moreover, $\mathbb{C}^{\times} \ltimes \mathbb{C}$ is an algebraic group, since it can be represented as a linear group by matrices of the form

$$
(a, x)=\left(\begin{array}{ll}
a & 0 \\
x & 1
\end{array}\right) \quad\left(a \in \mathbb{C}^{\times}, x \in \mathbb{C}\right)
$$

(and multiplication, unit and inverse element become usual operations with matrices).

Stereotype algebras $\mathcal{R}\left(\mathbb{C}^{\times} \ltimes \mathbb{C}\right)$ and $\mathcal{R}^{\star}\left(\mathbb{C}^{\times} \ltimes \mathbb{C}\right)$. By symbol $\mathcal{R}\left(\mathbb{C}^{\times} \ltimes \mathbb{C}\right)$ we, as usual, denote the algebra of polynomials on an algebraic group $\mathbb{C}^{\times} \ltimes \mathbb{C}$. According to the general approach of $\S$ a (b)] we endow the space $\mathcal{R}\left(\mathbb{C}^{\times} \ltimes \mathbb{C}\right)$ with the strongest locally convex topology. The dual space of currents $\mathcal{R}^{\star}\left(\mathbb{C}^{\times} \ltimes \mathbb{C}\right)$ is an algebra with respect to the usual convolution of functionals (3.14). Like any other dual space to a stereotype space, it is endowed with the topology of uniform convergence on compact sets in $\mathcal{R}\left(\mathbb{C}^{\times} \ltimes \mathbb{C}\right)$. In this case this is equivalent to the $\mathcal{R}\left(\mathbb{C}^{\times} \ltimes \mathbb{C}\right)$-weak topology. 
Recall that by $z^{n}$ and $t^{k}$ we denote basis monomials in the spaces $\mathcal{R}\left(\mathbb{C}^{\times}\right)$and $\mathcal{R}(\mathbb{C})$ (we defined them by formulas (3.30) and (3.52) ). In accordance with the common notation (1.24), it is reasonable to denote the basis monomials in the space of functions $\mathcal{R}^{\star}\left(\mathbb{C}^{\times} \ltimes \mathbb{C}\right)$ by symbol $z^{n} \square t^{k}$ :

$$
\left(z^{n} \boxminus t^{k}\right)(a, x):=a^{n} \cdot x^{k}, \quad a \in \mathbb{C}^{\times}, x \in \mathbb{C}
$$

Similarly, extending the old notations $\zeta_{n}$ and $\tau^{k}$ from (3.31) and (3.59), we denote by $\zeta_{n}$ 困 $\tau^{k}$ the functional on $\mathcal{R}\left(\mathbb{C}^{\times} \ltimes \mathbb{C}\right)$ of taking the $n$-th coefficient of Laurent series with respect to the first variable and at the same time the $k$-th derivative in the point $(1,0)$ with respect to the second variable:

$$
\zeta_{n} \circledast \tau^{k}(u)=\left.\int_{0}^{1} e^{-2 \pi i n t} \frac{\mathrm{d}^{k}}{\mathrm{~d} x^{k}} u\left(x, e^{2 \pi i t}\right)\right|_{x=0} \mathrm{~d} t=\left.\frac{\mathrm{d}^{k}}{\mathrm{~d} x^{k}} \int_{0}^{1} e^{-2 \pi i n t} u\left(x, e^{2 \pi i t}\right) \mathrm{d} t\right|_{x=0}
$$

Proposition 7.4. 1) The functions $\left\{z^{n} \boxminus t^{k} ; n \in \mathbb{Z}, k \in \mathbb{N}\right\}$ form an algebraic basis in the space $\mathcal{R}\left(\mathbb{C}^{\times} \ltimes \mathbb{C}\right)$ of polynomials on $\mathbb{C}^{\times} \ltimes \mathbb{C}$ : every polynomial $u \in \mathcal{R}\left(\mathbb{C}^{\times} \ltimes \mathbb{C}\right)$ is uniquely decomposed into the series

$$
u=\sum_{k \in \mathbb{N}, n \in \mathbb{Z}} u_{n, k} \cdot z^{n} \boxminus t^{k}, \quad \operatorname{card}\left\{(n, k): u_{n, k} \neq 0\right\}<\infty,
$$

where the coefficients can be computed by formula

$$
u_{n, k}=\frac{1}{k !} \cdot \zeta_{n} \circledast \tau^{k}(u)
$$

The correspondence $u \leftrightarrow\left\{u_{n, k} ; n \in \mathbb{Z}, k \in \mathbb{N}\right\}$ establishes an isomorphism of topological vector spaces

$$
\mathcal{R}\left(\mathbb{C}^{\times} \ltimes \mathbb{C}\right) \cong \mathbb{C}_{\mathbb{Z} \times \mathbb{N}}
$$

2) The functionals $\left\{\zeta_{n} ⿴ \tau^{k} ; n \in \mathbb{Z}, k \in \mathbb{N}\right\}$ form a basis in the stereotype space $\mathcal{R}^{\star}\left(\mathbb{C}^{\times} \ltimes \mathbb{C}\right)$ : every functional $\alpha \in \mathcal{R}^{\star}\left(\mathbb{C}^{\times} \ltimes \mathbb{C}\right)$ is uniquely decomposed into a (converging in the space $\mathcal{R}^{\star}\left(\mathbb{C}^{\times} \ltimes \mathbb{C}\right)$ ) series

$$
\alpha=\sum_{k \in \mathbb{N}, n \in \mathbb{Z}} \alpha_{n, k} \cdot \zeta_{n} \Psi \tau^{k}
$$

where the coefficients can be computed by formula

$$
\alpha_{n, k}=\frac{1}{k !} \cdot \alpha\left(z^{n} \boxminus t^{k}\right)
$$

The correspondence $\alpha \leftrightarrow\left\{\alpha_{n, k} ; n \in \mathbb{Z}, k \in \mathbb{N}\right\}$ establishes an isomorphism of topological vector spaces

$$
\mathcal{R}^{\star}\left(\mathbb{C}^{\times} \ltimes \mathbb{C}\right) \cong \mathbb{C}^{\mathbb{Z} \times \mathbb{N}}
$$

3) The bases $\left\{z^{n} \boxminus t^{k} ; n \in \mathbb{Z}, k \in \mathbb{N}\right\}$ and $\left\{\zeta_{n} \otimes \tau^{k} ; n \in \mathbb{Z}, k \in \mathbb{N}\right\}$ are dual to each other up to the constant $k !$

$$
\left\langle z^{m} \boxminus t^{k}, \zeta_{n} * \tau^{l}\right\rangle=\left\langle z^{m}, \zeta_{n}\right\rangle \cdot\left\langle t^{k}, \tau^{l}\right\rangle= \begin{cases}0, & (m, k) \neq(n, l) \\ k !, & (m, k)=(n, l)\end{cases}
$$

and the action of functionals $\alpha \in \mathcal{R}^{\star}\left(\mathbb{C}^{\times} \ltimes \mathbb{C}\right)$ on polynomials $u \in \mathcal{R}\left(\mathbb{C}^{\times} \ltimes \mathbb{C}\right)$ is described by the formula

$$
\langle u, \alpha\rangle=\sum_{n \in \mathbb{Z}, k \in \mathbb{N}} u_{n, k} \cdot \alpha_{n, k} \cdot k !
$$

Remark 7.1. The functional $\zeta_{n}$ 头 $\tau^{k}$ can be represented as the convolution of two components $\zeta_{n}$ and $\tau^{k}$ (and the order of their multiplication becomes important here): if we denote by $Z_{n}$ the functional of taking the $n$-th coefficient of Laurent series with respect to the first variable in the point $(1,0)$,

$$
Z_{n}(u)=\zeta_{n} ⿴ \tau^{0}(u)=\frac{1}{2 \pi i} \int_{|z|=1} \frac{u(z, 0)}{z^{n+1}} \mathrm{~d} z=\int_{0}^{1} e^{-2 \pi i n t} u\left(e^{2 \pi i t}, 0\right) \mathrm{d} t, \quad u \in \mathcal{R}\left(\mathbb{C}^{\times} \ltimes \mathbb{C}\right),
$$


and by $T^{k}$ the functional of taking the $k$-th derivative with respect to the second variable in the point $(1,0)$,

$$
T^{k}(u)=\zeta_{0} \circledast \tau^{k}(u)=\left.\frac{\mathrm{d}^{k}}{\mathrm{~d} x^{k}} u(1, x)\right|_{x=0}, \quad u \in \mathcal{R}\left(\mathbb{C}^{\times} \ltimes \mathbb{C}\right),
$$

then the following equalities hold:

$$
Z_{n} * T^{k}=\zeta_{n} \circledast \tau^{k}, \quad T^{k} * Z_{n}=Z_{n-k} * T^{k}=\zeta_{n-k} \circledast \tau^{k}
$$

For the proof we can use formulas (3.15): first,

$$
\begin{aligned}
& \delta^{a} * T^{k}=a \cdot T^{k} \\
& \Downarrow \\
& \left(\delta^{\left(e^{2 \pi i t}, 0\right)} * T^{k}\right)(u)=\left(\left(e^{2 \pi i t}, 0\right) \cdot T^{k}\right)(u)=T^{k}\left(u \cdot\left(e^{2 \pi i t}, 0\right)\right)= \\
& =\left.\frac{\mathrm{d}^{k}}{\mathrm{~d} x^{k}} u\left(\left(e^{2 \pi i t}, 0\right) \cdot(1, x)\right)\right|_{x=0}=\left.\frac{\mathrm{d}^{k}}{\mathrm{~d} x^{k}} u\left(e^{2 \pi i t}, x\right)\right|_{x=0} \\
& \Downarrow \\
& \left(Z_{n} * T^{k}\right)(u)=\left(\int_{0}^{1} e^{-2 \pi i n t} \cdot \delta^{\left(e^{2 \pi i t}, 0\right)} \mathrm{d} t * T^{k}\right)(u)=\left(\int_{0}^{1} e^{-2 \pi i n t} \cdot \delta^{\left(e^{2 \pi i t}, 0\right) * T^{k}} \mathrm{~d} t\right)(u)= \\
& =\int_{0}^{1}\left(e^{-2 \pi i n t} \cdot \delta^{\left(e^{2 \pi i t}, 0\right)} * T^{k}\right)(u) \mathrm{d} t=\left.\int_{0}^{1} e^{-2 \pi i n t} \cdot \frac{\mathrm{d}^{k}}{\mathrm{~d} x^{k}} u\left(e^{2 \pi i t}, x\right)\right|_{x=0} \mathrm{~d} t
\end{aligned}
$$

And, second,

$$
\begin{gathered}
T^{k} * \delta^{a}=T^{k} \cdot a \\
\Downarrow \\
\left(T^{k} * \delta^{\left(e^{2 \pi i t}, 0\right)}\right)(u)=\left(T^{k} \cdot\left(e^{2 \pi i t}, 0\right)\right)(u)=T^{k}\left(\left(e^{2 \pi i t}, 0\right) \cdot u\right)=\left.\frac{\mathrm{d}^{k}}{\mathrm{~d} x^{k}} u\left((1, x) \cdot\left(e^{2 \pi i t}, 0\right)\right)\right|_{x=0}= \\
=\left.\frac{\mathrm{d}^{k}}{\mathrm{~d} x^{k}} u\left(e^{2 \pi i t}, x \cdot e^{2 \pi i t}\right)\right|_{x=0}=\left.\left(e^{2 \pi i t}\right)^{k} \cdot \frac{\mathrm{d}^{k}}{\mathrm{~d} y^{k}} u\left(e^{2 \pi i t}, y\right)\right|_{y=0}=(7.57)=e^{2 \pi i k t} \cdot\left(\delta^{\left(e^{2 \pi i t}, 0\right)} * T^{k}\right)(u) \\
\Downarrow \\
T^{k} * \delta^{\left(e^{2 \pi i t}, 0\right)}=e^{2 \pi i k t} \cdot \delta^{\left(e^{2 \pi i t}, 0\right)} * T^{k} \\
\Downarrow \\
T^{k} * Z_{n}=T^{k} * \int_{0}^{1} e^{-2 \pi i n t} \cdot \delta^{\left(e^{2 \pi i t}, 0\right)} \mathrm{d} t=\int_{0}^{1} e^{-2 \pi i n t} \cdot T^{k} * \delta^{\left(e^{2 \pi i t}, 0\right)} \mathrm{d} t=(7.58) \\
=\int_{0}^{1} e^{-2 \pi i n t} \cdot e^{2 \pi i k t} \cdot \delta^{\left(e^{2 \pi i t}, 0\right)} * T^{k} \mathrm{~d} t=\int_{0}^{1} e^{-2 \pi i(n-k) t} \cdot \delta^{\left(e^{2 \pi i t}, 0\right)} * T^{k} \mathrm{~d} t= \\
=\int_{0}^{1} e^{-2 \pi i(n-k) t} \cdot \delta^{\left(e^{2 \pi i t}, 0\right)} \mathrm{d} t * T^{k}=Z_{n-k} * T^{k}
\end{gathered}
$$


Stereotype algebras $\mathcal{O}\left(\mathbb{C}^{\times} \ltimes \mathbb{C}\right)$ and $\mathcal{O}^{\star}\left(\mathbb{C}^{\times} \ltimes \mathbb{C}\right)$. As always, we endow the algebra $\mathcal{O}\left(\mathbb{C}^{\times} \ltimes \mathbb{C}\right)$ of holomorphic functions on $\mathbb{C}^{\times} \ltimes \mathbb{C}$ with the topology of uniform convergence on compact sets in $\mathbb{C}^{\times} \ltimes \mathbb{C}$. Its dual algebra $\mathcal{O}^{\star}\left(\mathbb{C}^{\times} \ltimes \mathbb{C}\right)$ is endowed with the topology of uniform convergence on compact sets in $\mathcal{O}\left(\mathbb{C}^{\times} \ltimes \mathbb{C}\right)$, and the multiplication there is the usual convolution (3.14).

Proposition 7.5. 1) The functions $\left\{z^{n} \square t^{k} ; n \in \mathbb{Z}, k \in \mathbb{N}\right\}$ form a basis in the stereotype space $\mathcal{O}\left(\mathbb{C}^{\times} \ltimes \mathbb{C}\right)$ of holomorphic functions on $\mathbb{C}^{\times} \ltimes \mathbb{C}$ : every function $u \in \mathcal{O}\left(\mathbb{C}^{\times} \ltimes \mathbb{C}\right)$ can be uniquely represented as a sum of a (converging in $\mathcal{O}\left(\mathbb{C}^{\times} \ltimes \mathbb{C}\right)$ ) series

$$
u=\sum_{k \in \mathbb{N}, n \in \mathbb{Z}} u_{n, k} \cdot z^{n} \boxminus t^{k}, \quad \forall C>0 \quad \sum_{k \in \mathbb{N}, n \in \mathbb{Z}}\left|u_{k, n}\right| \cdot C^{k+|n|}<\infty,
$$

where the coefficients (continuously depend on $u$ and) can be computed by formula

$$
u_{n, k}=\frac{1}{k !} \cdot \zeta_{n} ⿴ \tau^{k}(u)
$$

The topology of $\mathcal{O}\left(\mathbb{C}^{\times} \ltimes \mathbb{C}\right)$ can be described by seminorms:

$$
\|u\|_{C}=\sum_{k \in \mathbb{N}, n \in \mathbb{Z}}\left|u_{k, n}\right| \cdot C^{k+|n|}, \quad C \geqslant 1
$$

2) The functionals $\left\{\zeta_{n} \circledast \tau^{k} ; n \in \mathbb{Z}, k \in \mathbb{N}\right\}$ form a basis in the space $\mathcal{O}^{\star}\left(\mathbb{C}^{\times} \times \mathbb{C}\right)$ : every functional $\alpha \in \mathcal{O}^{\star}\left(\mathbb{C}^{\times} \ltimes \mathbb{C}\right)$ can be uniquely represented as a sum of a (converging in $\mathcal{O}^{\star}\left(\mathbb{C}^{\times} \ltimes \mathbb{C}\right)$ ) series

$$
\alpha=\sum_{k \in \mathbb{N}, n \in \mathbb{Z}} \alpha_{n, k} \cdot \zeta_{n} ⿴ \tau^{k}
$$

where the coefficients (continuously depend on $\alpha$ and) can be computed by formula

$$
\alpha_{n, k}=\frac{1}{k !} \cdot \alpha\left(z^{n} \boxminus t^{k}\right)
$$

The topology of the space $\mathcal{O}^{\star}\left(\mathbb{C}^{\times} \ltimes \mathbb{C}\right)$ can be described by seminorms:

$$
\||\alpha|\|_{r}=\sum_{n \in \mathbb{Z}, k \in \mathbb{N}} r_{n, k} \cdot\left|\alpha_{n, k}\right| \cdot k !, \quad r_{n, k} \geqslant 0: \forall C \geqslant 1 \sum_{n \in \mathbb{Z}, k \in \mathbb{N}} r_{k, n} \cdot C^{k+|n|}<\infty
$$

3) The bases $\left\{z^{n} \square t^{k} ; n \in \mathbb{Z}, k \in \mathbb{N}\right\}$ and $\left\{\zeta_{n} \circledast \tau^{k} ; n \in \mathbb{Z}, k \in \mathbb{N}\right\}$ are dual to each other up to the constant $k !$ :

$$
\left\langle z^{m} \sqcup t^{k}, \zeta_{n} * \tau^{l}\right\rangle=\left\langle z^{m}, \zeta_{n}\right\rangle \cdot\left\langle t^{k}, \tau^{l}\right\rangle= \begin{cases}0, & (m, k) \neq(n, l) \\ k !, & (m, k)=(n, l)\end{cases}
$$

and the action of functionals $\alpha \in \mathcal{O}^{\star}\left(\mathbb{C}^{\times} \ltimes \mathbb{C}\right)$ on functions $u \in \mathcal{O}\left(\mathbb{C}^{\times} \ltimes \mathbb{C}\right)$ is described by formula

$$
\langle u, \alpha\rangle=\sum_{n \in \mathbb{Z}, k \in \mathbb{N}} u_{n, k} \cdot \alpha_{n, k} \cdot k !
$$

$\mathcal{R}\left(\mathbb{C}^{\times} \ltimes \mathbb{C}\right), \mathcal{R}^{\star}\left(\mathbb{C}^{\times} \ltimes \mathbb{C}\right), \mathcal{O}\left(\mathbb{C}^{\times} \ltimes \mathbb{C}\right)$ and $\mathcal{O}^{\star}\left(\mathbb{C}^{\times} \ltimes \mathbb{C}\right)$ as Hopf algebras. The algebras $\mathcal{R}\left(\mathbb{C}^{\times} \ltimes \mathbb{C}\right)$ and $\mathcal{O}\left(\mathbb{C}^{\times} \ltimes \mathbb{C}\right)$, being standard functional algebras on groups, are endowed with the natural structure of Hopf algebras (we noted this general fact in Theorems 3.2 and 3.1). The following propositions describe the structure of these Hopf algebras.

Proposition 7.6. The algebra $\mathcal{R}\left(\mathbb{C}^{\times} \ltimes \mathbb{C}\right)$ (resp., algebra $\mathcal{O}\left(\mathbb{C}^{\times} \ltimes \mathbb{C}\right)$ ) is a nuclear Hopf-Brauner (resp., Hopf-Fréchet) algebra with the algebraic operations defined on basis elements $z^{n} \boxminus t^{k}$ by formulas

$$
z^{m} \boxminus t^{k} \cdot z^{n} \square t^{l}=z^{m+n} \boxminus t^{k+l} \quad 1_{\mathcal{R}(\mathbb{C} \times \ltimes \mathbb{C})}=z^{0} t^{0}
$$




$$
\begin{aligned}
& \varkappa\left(z^{n} \sqcup t^{k}\right)=\sum_{i=0}^{k}\left(\begin{array}{c}
k \\
i
\end{array}\right) \cdot z^{n} t^{i} \odot z^{n+i} t^{k-i} \\
& \sigma\left(z^{n} \sqcup t^{k}\right)=(-1)^{k} z^{-k-n} t^{k}
\end{aligned} \quad \varepsilon\left(z^{n} \boxminus t^{k}\right)= \begin{cases}0, & (n, k) \neq(0,0) \\
1, & (n, k)=(0,0)\end{cases}
$$

As a corollary, the general formula of multiplication in $\mathcal{R}\left(\mathbb{C}^{\times} \ltimes \mathbb{C}\right)$ is as follows:

$$
u \cdot v=\sum_{n \in \mathbb{Z}, k \in \mathbb{N}}\left(\sum_{j=0}^{k} \sum_{i \in \mathbb{Z}} u_{i, j} \cdot v_{n-i, k-j}\right) \cdot z^{n} \boxminus t^{k} .
$$

Proof. The comultiplication, counit and antipode can be computed by formulas (3.5)-(3.7). For instance, the comultiplication:

$$
\begin{aligned}
& \tilde{\varkappa}\left(z^{n} \boxminus t^{k}\right)((a, x),(b, y))=\left(z^{n} \boxminus t^{k}\right)((a, x) \cdot(b, y))=\left(z^{n} \boxminus t^{k}\right)(a b, x b+y)=(a b)^{n}(x b+y)^{k}= \\
& =a^{n} b^{n} \sum_{i=0}^{k}\left(\begin{array}{c}
k \\
i
\end{array}\right) x^{i} b^{i} y^{k-i}=\sum_{i=0}^{k}\left(\begin{array}{c}
k \\
i
\end{array}\right) a^{n} x^{i} b^{n+i} y^{k-i}=\sum_{i=0}^{k}\left(\begin{array}{c}
k \\
i
\end{array}\right)\left(z^{n} \boxminus t^{i}\right) \sqcup\left(z^{n+i} \boxminus t^{k-i}\right)((a, x),(b, y)) \\
& \Downarrow \\
& \widetilde{\varkappa}\left(z^{n} \boxminus t^{k}\right)=\sum_{i=0}^{k}\left(\begin{array}{c}
k \\
i
\end{array}\right) \cdot\left(z^{n} \sqcup t^{i}\right) \sqcup\left(z^{n+i} \sqcup t^{k-i}\right) \\
& \Downarrow \\
& \varkappa\left(z^{n} \boxminus t^{k}\right)=\sum_{i=0}^{k}\left(\begin{array}{c}
k \\
i
\end{array}\right) \cdot z^{n} \boxminus t^{i} \odot z^{n+i} \boxminus t^{k-i}
\end{aligned}
$$

Proposition 7.7. The algebra $\mathcal{R}^{\star}\left(\mathbb{C}^{\times} \ltimes \mathbb{C}\right)$ (resp., algebra $\mathcal{O}^{\star}\left(\mathbb{C}^{\times} \ltimes \mathbb{C}\right)$ ) is a nuclear Hopf-Fréchet (resp., Hopf-Brauner) algebra with the algebraic operations defined on basis elements $\left.\zeta_{n} ⿴ 囗 ⿱ 一 一\right) \tau^{k}$ by formulas

$$
\begin{array}{ll}
\left(\zeta_{m} \circledast \tau^{k}\right) *\left(\zeta_{n} \circledast \tau^{l}\right)= \begin{cases}\zeta_{m} \circledast \tau^{k+l}, & m+k=n \\
0, & m+k \neq n\end{cases} & 1_{\mathcal{R}^{\star}\left(\mathbb{C}^{\star} \ltimes \mathbb{C}\right)}=\sum_{n \in \mathbb{Z}} \zeta_{n} \circledast \tau^{0} \\
\varkappa\left(\zeta_{n} \circledast \tau^{k}\right)=\sum_{m \in \mathbb{Z}} \sum_{i=0}^{k}\left(\begin{array}{c}
k \\
i
\end{array}\right) \cdot \zeta_{m} \circledast \tau^{i} \circledast \zeta_{n-m} \circledast \tau^{k-i} & \varepsilon\left(\zeta_{n} \circledast \tau^{k}\right)= \begin{cases}1, & (n, k)=(0,0) \\
0, & (n, k) \neq(0,0)\end{cases} \\
\sigma\left(\zeta^{n} \circledast \tau^{k}\right)=(-1)^{k} \cdot \zeta_{-n-k} \circledast \tau^{k} &
\end{array}
$$

As a corollary, the general formula of multiplication in $\mathcal{R}^{\star}\left(\mathbb{C}^{\times} \ltimes \mathbb{C}\right)$ is as follows:

$$
\left.\alpha * \beta=\sum_{n \in \mathbb{Z}, k \in \mathbb{N}}\left(\sum_{j=0}^{k} \alpha_{n, j} \cdot \beta_{n+j, k-j}\right) \cdot \zeta_{n} ⿴ 囗 ⿱ 一 一\right) \tau^{k} .
$$

Proof. All those formulas appear as duals of (7.67)-(7.69). For example, the comultiplication is computed as follows:

$$
\begin{array}{r}
\left\langle u \odot v, \varkappa\left(\zeta_{n} \circledast \tau^{k}\right)\right\rangle=\left\langle u \cdot v, \zeta_{n} \circledast \tau^{k}\right\rangle=\left\langle\sum_{r \in \mathbb{Z}, l \in \mathbb{N}}\left(\sum_{i=0}^{l} \sum_{m \in \mathbb{Z}} u_{m, i} \cdot v_{r-m, l-i}\right) \cdot z^{r} t^{l}, \zeta_{n} \circledast \tau^{k}\right\rangle= \\
=k ! \cdot \sum_{i=0}^{k} \sum_{m \in \mathbb{Z}} u_{m, i} \cdot v_{n-m, k-i}=k ! \cdot \sum_{i=0}^{k} \sum_{m \in \mathbb{Z}} \frac{1}{i !}\left\langle u, \zeta_{m} \circledast \tau^{i}\right\rangle \cdot \frac{1}{(k-i) !}\left\langle v, \zeta_{n-m} \circledast \tau^{k-i}\right\rangle=
\end{array}
$$




$$
\begin{gathered}
=\sum_{m \in \mathbb{Z}} \sum_{i=0}^{k}\left(\begin{array}{c}
k \\
i
\end{array}\right) \cdot\left\langle u \odot v, \zeta_{m} \circledast \tau^{i} \circledast \zeta_{n-m} \circledast \tau^{k-i}\right\rangle=\left\langle u \odot v, \sum_{m \in \mathbb{Z}} \sum_{i=0}^{k}\left(\begin{array}{c}
k \\
i
\end{array}\right) \cdot \zeta_{m} \circledast \tau^{i} \circledast \zeta_{n-m} \circledast \tau^{k-i}\right\rangle \\
\Downarrow \\
\varkappa\left(\zeta_{n} \circledast \tau^{k}\right)=\sum_{m \in \mathbb{Z}} \sum_{i=0}^{k}\left(\begin{array}{c}
k \\
i
\end{array}\right) \cdot \zeta_{m} \circledast \tau^{i} \circledast \zeta_{n-m} \circledast \tau^{k-i}
\end{gathered}
$$

Hopf algebras $\mathcal{R}_{q}\left(\mathbb{C}^{\times} \ltimes \mathbb{C}\right), \mathcal{R}_{q}^{\star}\left(\mathbb{C}^{\times} \ltimes \mathbb{C}\right), \mathcal{O}_{q}\left(\mathbb{C}^{\times} \ltimes \mathbb{C}\right), \mathcal{O}_{q}^{\star}\left(\mathbb{C}^{\times} \ltimes \mathbb{C}\right)$. The quantum group ' $a z+b$ ' can be defined as Hopf algebra $\mathcal{R}\left(\mathbb{C}^{\times} \ltimes \mathbb{C}\right)$, where the algebraic operations are deformed in some special way. We describe here this deformation, and together with the algebra $\mathcal{R}\left(\mathbb{C}^{\times} \ltimes \mathbb{C}\right)$ we shall consider the algebra $\mathcal{O}\left(\mathbb{C}^{\times} \ltimes \mathbb{C}\right)$. Both these constructions will be useful below in Theorem 7.8 .

The constructions starts with the choice of a constant $q \in \mathbb{C}^{\times}$.

Proposition 7.8. On the stereotype space $\mathcal{R}\left(\mathbb{C}^{\times} \ltimes \mathbb{C}\right)$ (respectively, $\mathcal{O}\left(\mathbb{C}^{\times} \ltimes \mathbb{C}\right)$ ) there exists a unique structure of rigid stereotype Hopf algebra with the algebraic operations defined on basis elements $z^{n} \square t^{k}$ by formulas

$$
\begin{array}{ll}
z^{m} \boxminus t^{k} \cdot z^{n} \boxminus t^{l}=q^{k n} \cdot z^{m+n} \boxminus t^{k+l} & 1_{\mathcal{R}(\mathbb{C} \times \ltimes \mathbb{C})}=z^{0} t^{0} \\
\varkappa\left(z^{n} \boxminus t^{k}\right)=\sum_{i=0}^{k}\left(\begin{array}{c}
k \\
i
\end{array}\right)_{q} \cdot z^{n} \boxminus t^{i} \odot z^{n+i} \boxminus t^{k-i} & \varepsilon\left(z^{n} \boxminus t^{k}\right)= \begin{cases}0, & (n, k) \neq(0,0) \\
1, & (n, k)=(0,0)\end{cases} \\
\sigma\left(z^{n} \boxminus t^{k}\right)=(-1)^{k} \cdot q^{-\frac{k(k+1)}{2}-k n} \cdot z^{-k-n} \boxminus t^{k} &
\end{array}
$$

The space $\mathcal{R}\left(\mathbb{C}^{\times} \ltimes \mathbb{C}\right)$ (respectively, $\mathcal{O}\left(\mathbb{C}^{\times} \ltimes \mathbb{C}\right)$ ) with such a structure of Hopf algebra is defined by $\mathcal{R}_{q}\left(\mathbb{C}^{\times} \ltimes \mathbb{C}\right)$ (respectively, by $\mathcal{O}_{q}\left(\mathbb{C}^{\times} \ltimes \mathbb{C}\right)$ ). Besides this,

1) the general formula of multiplication in $\mathcal{R}_{q}\left(\mathbb{C}^{\times} \ltimes \mathbb{C}\right)$ (respectively, in $\mathcal{O}_{q}\left(\mathbb{C}^{\times} \ltimes \mathbb{C}\right)$ ) has the form

$$
u \cdot v=\sum_{n \in \mathbb{Z}, k \in \mathbb{N}}\left(\sum_{i=0}^{k} \sum_{m \in \mathbb{Z}} q^{i(n-m)} \cdot u_{m, i} \cdot v_{n-m, k-i}\right) \cdot z^{n} \boxminus t^{k} .
$$

2) The mapping

$$
z^{n} \bullet t^{k} \mapsto z^{n} \odot t^{k}
$$

establishes an isomorphism between $\mathcal{R}_{q}\left(\mathbb{C}^{\times} \ltimes \mathbb{C}\right)$ (respectively, $\mathcal{O}_{q}\left(\mathbb{C}^{\times} \ltimes \mathbb{C}\right)$ ) and the Hopf algebra of skew polynomials (entire functions) with coefficients in $\mathcal{R}\left(\mathbb{C}^{\times}\right)$(respectively, in $\mathcal{O}\left(\mathbb{C}^{\times}\right)$) with respect to the quantum pair $\left(z, \delta^{q}\right)$ from Proposition 7.1:

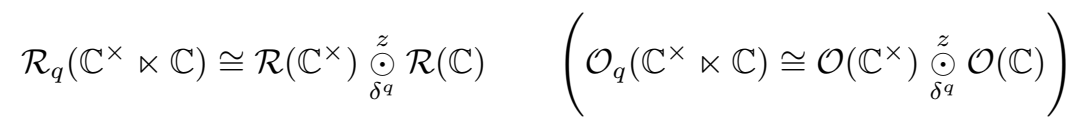

3) For $q=1$ the Hopf algebra $\mathcal{R}_{q}\left(\mathbb{C}^{\times} \ltimes \mathbb{C}\right)$ (respectively, $\mathcal{O}_{q}\left(\mathbb{C}^{\times} \ltimes \mathbb{C}\right)$ ) turns into the Hopf algebra $\mathcal{R}\left(\mathbb{C}^{\times} \ltimes \mathbb{C}\right)$ (respectively, $\mathcal{O}\left(\mathbb{C}^{\times} \ltimes \mathbb{C}\right)$ ) with the structure of Hopf algebra described on page 117 ;

$$
\mathcal{R}\left(\mathbb{C}^{\times} \ltimes \mathbb{C}\right)=\mathcal{R}_{1}\left(\mathbb{C}^{\times} \ltimes \mathbb{C}\right) \quad\left(\mathcal{O}\left(\mathbb{C}^{\times} \ltimes \mathbb{C}\right)=\mathcal{O}_{1}\left(\mathbb{C}^{\times} \ltimes \mathbb{C}\right)\right)
$$


Proof. Here everything starts from formulas (7.79): the mapping $z^{n} \odot t^{k} \mapsto z^{n} \odot t^{k}$ establishes an isomorphism of stereotype spaces

$$
\mathcal{R}_{q}\left(\mathbb{C}^{\times} \ltimes \mathbb{C}\right) \cong \mathcal{R}\left(\mathbb{C}^{\times}\right) \odot \mathcal{R}(\mathbb{C})
$$

(this is exactly the isomorphism, which for general case is described by the identity (3.9)). This isomorphism induces on $\mathcal{R}\left(\mathbb{C}^{\times} \ltimes \mathbb{C}\right.$ ) the structure of rigid Hopf algebra from $\mathcal{R}\left(\mathbb{C}^{\times}\right) \stackrel{z}{\stackrel{z}{\odot}} \mathcal{R}(\mathbb{C})$ (where this structure is defined by formulas (7.14)-(7.18) $)$. In this isomorphism the formulas (7.14)-(7.18) turn into formulas (7.75)-(7.77). To derive them we need to use the first formula in (7.12):

$$
\mathrm{M}_{\delta^{q}}^{\star}(z)=q \cdot z
$$

This implies

$$
\left(\mathrm{M}_{\delta^{q}}^{\star}\right)^{k}\left(z^{n}\right)=q^{k n} \cdot z^{n}
$$

Then, for instance, the formula of multiplication (7.75) is derived from (17.14) as follows:

$$
z^{m} \odot t^{k} \cdot z^{n} \odot t^{l}=z^{m} \cdot\left(\mathrm{M}_{\delta^{q}}^{\star}\right)^{k}\left(z^{n}\right) \cdot t^{k+l}=z^{m} \cdot q^{k n} \cdot z^{n} \odot t^{k+l}=q^{k n} \cdot z^{k+n} \odot t^{k+l}
$$

The remaining formulas are deduced by analogy. The general formula for multiplication (7.78) follows from (7.75):

$$
\begin{gathered}
u \cdot v=\left(\sum_{m \in \mathbb{Z}, k \in \mathbb{N}} u_{m, k} \cdot z^{m} \boxminus t^{k}\right) \cdot\left(\sum_{n \in \mathbb{Z}, l \in \mathbb{N}} v_{n, l} \cdot z^{n} \boxminus t^{l}\right)=\sum_{m \in \mathbb{Z}, k \in \mathbb{N}} \sum_{n \in \mathbb{Z}, l \in \mathbb{N}} u_{m, k} \cdot v_{n, l} \cdot z^{m} \bullet t^{k} \cdot z^{n} \boxminus t^{l}= \\
=\sum_{m \in \mathbb{Z}, k \in \mathbb{N}} \sum_{n \in \mathbb{Z}, l \in \mathbb{N}} u_{m, k} \cdot v_{n, l} \cdot q^{k n} \cdot z^{m+n} t^{k+l}=\left(\begin{array}{c}
n=r-m \\
l=s-k
\end{array}\right)= \\
=\sum_{r \in \mathbb{Z}, s \in \mathbb{N}} \sum_{m \in \mathbb{Z}, k \in \mathbb{N}} q^{k(r-m)} \cdot u_{m, k} \cdot v_{r-m, s-k} \cdot z^{m+n} t^{k+l}
\end{gathered}
$$

It remains to add that for $q=1$ the formulas (7.75)-(7.77) turn into formulas (7.67)-(7.69), so the Hopf algebra $\mathcal{R}_{q}\left(\mathbb{C}^{\times} \ltimes \mathbb{C}\right)$ (respectively, $\mathcal{O}_{q}\left(\mathbb{C}^{\times} \ltimes \mathbb{C}\right)$ ) turns into Hopf algebra $\mathcal{R}\left(\mathbb{C}^{\times} \ltimes \mathbb{C}\right)$ (respectively, $\left.\mathcal{O}\left(\mathbb{C}^{\times} \ltimes \mathbb{C}\right)\right)$.

Proposition 7.9. On the stereotype space $\mathcal{R}^{\star}\left(\mathbb{C}^{\times} \ltimes \mathbb{C}\right)$ (respectively, $\mathcal{O}^{\star}\left(\mathbb{C}^{\times} \ltimes \mathbb{C}\right)$ ) there exists a unique structure of rigid stereotype Hopf algebra with algebraic operations defined on basis elements $\left.\zeta_{n} ⿴ 囗 ⿱ 一 一\right) \tau^{k}$ by formulas

$$
\begin{array}{ll}
\left(\zeta_{m} \circledast \tau^{k}\right) *\left(\zeta_{n} \circledast \tau^{l}\right)= \begin{cases}\zeta_{m} \circledast \tau^{k+l}, & m=n-k \\
0, & m \neq n-k\end{cases} & 1_{\mathcal{R}^{\star}\left(\mathbb{C}^{\star} \ltimes \mathbb{C}\right)}=\sum_{n \in \mathbb{Z}} \zeta_{n} \circledast \tau^{0} \\
\varkappa\left(\zeta_{n} \circledast \tau^{k}\right)=\sum_{m \in \mathbb{Z}} \sum_{i=0}^{k}\left(\begin{array}{l}
k \\
i
\end{array}\right)_{q} \cdot q^{i(n-m)} \cdot \zeta_{m} \circledast \tau^{i} \circledast \zeta_{n-m} \circledast \tau^{k-i} & \varepsilon\left(\zeta_{n} \circledast \tau^{k}\right)= \begin{cases}1, & k=0 \\
0, & k>0\end{cases} \\
\sigma\left(\zeta^{n} \circledast \tau^{k}\right)=(-1)^{k} \cdot q^{-\frac{k(k+1)}{2}+k(n+k)} \zeta_{-n-k} \circledast \tau^{k} &
\end{array}
$$

The space $\mathcal{R}^{\star}\left(\mathbb{C}^{\times} \ltimes \mathbb{C}\right)$ (respectively, $\mathcal{O}^{\star}\left(\mathbb{C}^{\times} \ltimes \mathbb{C}\right)$ ) with this structure of Hopf algebra is denoted by $\mathcal{R}_{q}^{\star}\left(\mathbb{C}^{\times} \ltimes \mathbb{C}\right)$ (respectively, $\mathcal{O}_{q}^{\star}\left(\mathbb{C}^{\times} \ltimes \mathbb{C}\right)$ ). Besides this,

1) the general formula for multiplication in $\mathcal{R}_{q}^{\star}\left(\mathbb{C}^{\times} \ltimes \mathbb{C}\right)$ (respectively, in $\mathcal{O}_{q}^{\star}\left(\mathbb{C}^{\times} \ltimes \mathbb{C}\right)$ ) has the form

$$
\alpha * \beta=\sum_{n \in \mathbb{Z}, k \in \mathbb{N}}\left(\sum_{i=0}^{k} \alpha_{n, i} \cdot \beta_{n+i, k-i}\right) \cdot \zeta_{n} \circledast \tau^{k} .
$$


2) the mapping

$$
\zeta_{n} \circledast \tau^{k} \mapsto \zeta_{n} \circledast \tau^{k}
$$

establishes an isomorphism between $\mathcal{R}_{q}^{\star}\left(\mathbb{C}^{\times} \ltimes \mathbb{C}\right)$ (respectively, $\mathcal{O}_{q}^{\star}\left(\mathbb{C}^{\times} \ltimes \mathbb{C}\right)$ ) and the Hopf algebra of skew power series (respectively, analytic functionals) with coefficients in $\mathcal{R}^{\star}\left(\mathbb{C}^{\times}\right)$(respectively, in $\mathcal{O}^{\star}\left(\mathbb{C}^{\times}\right)$) with respect to the quantum pair $\left(\delta^{q}, z\right)$ from Proposition 7.1 .

$$
\mathcal{R}_{q}^{\star}\left(\mathbb{C}^{\times} \ltimes \mathbb{C}\right) \cong \mathcal{R}^{\star}\left(\mathbb{C}^{\times}\right) \underset{z}{\delta^{q}} \mathcal{R}^{\star}(\mathbb{C}), \quad \mathcal{O}_{q}^{\star}\left(\mathbb{C}^{\times} \ltimes \mathbb{C}\right) \cong \mathcal{O}^{\star}\left(\mathbb{C}^{\times}\right){\stackrel{\odot}{\delta^{q}}}_{\mathcal{O}^{\star}}(\mathbb{C})
$$

3) for $q=1$ the Hopf algebra $\mathcal{R}_{q}^{\star}\left(\mathbb{C}^{\times} \ltimes \mathbb{C}\right)$ (respectively, $\mathcal{O}_{q}^{\star}\left(\mathbb{C}^{\times} \ltimes \mathbb{C}\right)$ ) turns into the Hopf algebra $\mathcal{R}^{\star}\left(\mathbb{C}^{\times} \ltimes \mathbb{C}\right)$ (respectively, $\mathcal{O}^{\star}\left(\mathbb{C}^{\times} \ltimes \mathbb{C}\right)$ ) with the structure of Hopf algebra defined on page 117 ;

$$
\mathcal{R}^{\star}\left(\mathbb{C}^{\times} \ltimes \mathbb{C}\right)=\mathcal{R}_{1}^{\star}\left(\mathbb{C}^{\times} \ltimes \mathbb{C}\right) \quad\left(\mathcal{O}^{\star}\left(\mathbb{C}^{\times} \ltimes \mathbb{C}\right)=\mathcal{O}_{1}^{\star}\left(\mathbb{C}^{\times} \ltimes \mathbb{C}\right)\right)
$$

Proof. Here again everything is based on formulas (7.84): the mapping $\zeta_{n} \circledast \tau^{k} \mapsto \zeta_{n} \circledast \tau^{k}$ establishes an isomorphism of stereotype spaces

$$
\mathcal{R}_{q}^{\star}\left(\mathbb{C}^{\times} \ltimes \mathbb{C}\right) \cong \mathcal{R}^{\star}\left(\mathbb{C}^{\times}\right) \circledast \mathcal{R}^{\star}(\mathbb{C})
$$

(this is the dual isomorphism for (3.9) ). This isomorphism induces on $\mathcal{R}^{\star}\left(\mathbb{C}^{\star} \ltimes \mathbb{C}\right)$ a structure of rigid Hopf algebra from $\mathcal{R}^{\star}\left(\mathbb{C}^{\times}\right) \underset{z}{\circledast} \mathcal{R}^{\star}(\mathbb{C})$ (where this structure is defined by formulas (7.20)-(7.24)). Under this isomorphism formulas (7.20)-(7.24) turn into formulas (7.80)-(7.82). To derive them we can use two formulas:

$$
\mathrm{M}_{z}^{\star}\left(\zeta_{n}\right)=\zeta_{n-1}, \quad \delta^{q} * \zeta_{n}=q^{n} \cdot \zeta_{n}
$$

The first of them (it will be useful in proving formulas for multiplication and antipode), is deduced as follows:

$$
\begin{aligned}
&\left\langle u, \mathrm{M}_{z}^{\star}\left(\zeta_{n}\right)\right\rangle=\left\langle z \cdot u, \zeta_{n}\right\rangle=\left\langle z \cdot \sum_{m \in \mathbb{Z}} u_{m} \cdot z^{m}, \zeta_{n}\right\rangle=\left\langle\sum_{m \in \mathbb{Z}} u_{m} \cdot z^{m+1}, \zeta_{n}\right\rangle= \\
&=\left\langle\sum_{m \in \mathbb{Z}} u_{m-1} \cdot z^{m}, \zeta_{n}\right\rangle=u_{n-1}=\left\langle u, \zeta_{n-1}\right\rangle
\end{aligned}
$$

And the second one (it will be useful in proving the formula for comultiplication) as follows:

$$
\delta^{q} * \zeta_{n}=(7.13)=\sum_{m \in \mathbb{Z}} q^{m} \cdot \zeta_{m} * \zeta_{n}=(3.41)=q^{n} \cdot \zeta_{n}
$$

$\mathcal{R}_{q}\left(\mathbb{C}^{\times} \ltimes \mathbb{C}\right)$ as an algebra with generators and defining relations. It remains to explain why the constructed algebra $\mathcal{R}_{q}\left(\mathbb{C}^{\times} \ltimes \mathbb{C}\right)$ indeed can be identified with the quantum group ' $a z+b$ ', i.e. with the Hopf algebra which is usually denoted as ' $a z+b$ ' (see [48, 40, 47, 28]). Formally ' $a z+b$ ' is defined as the Hopf algebra with three generators $t, z, z^{-1}$ and the defining relations

$$
\begin{array}{lll}
t \cdot z=q \cdot z \cdot t & z \cdot z^{-1}=1 & 1=z^{-1} \cdot z \\
\varkappa(t)=t \otimes 1+z \otimes t, & \varkappa(z)=z \otimes z, & \varkappa\left(z^{-1}\right)=z^{-1} \otimes z^{-1} \\
\varepsilon(t)=0, & \varepsilon(z)=1, & \varepsilon\left(z^{-1}\right)=0 \\
\sigma(t)=-t \cdot z^{-1}, & \sigma(z)=z^{-1}, & \sigma\left(z^{-1}\right)=z,
\end{array}
$$


Proposition 7.10. The mapping

$$
z \mapsto z \boxminus 1, \quad z^{-1} \mapsto z^{-1} \boxminus 1, \quad t \mapsto 1 \boxminus t
$$

is uniquely extended to an isomorphism between Hopf algebras ' $a z+b$ ' and $\mathcal{R}_{q}\left(\mathbb{C}^{\times} \ltimes \mathbb{C}\right)$.

Proof. From (7.75) it follows that in $\mathcal{R}_{q}\left(\mathbb{C}^{\times} \ltimes \mathbb{C}\right)$ the following identities hold

$$
1 \boxminus t \cdot z \boxminus 1=q \cdot z \boxminus 1 \cdot 1 \boxminus t, \quad z \boxminus 1 \cdot z^{-1} \boxminus 1=1, \quad 1=z^{-1} \boxminus 1 \cdot z \boxminus 1
$$

in which one can recognize formulas (7.86), transformed by our mapping. This means that our mapping can be uniquely extended to some homomorphism of algebras $\varphi:{ }^{\prime} a z+b^{\prime} \rightarrow \mathcal{R}_{q}\left(\mathbb{C}^{\times} \ltimes \mathbb{C}\right)$. This homomorphism is a bijection since it turns the algebraic basis $\left\{z^{n} t^{k} ; n \in \mathbb{Z}, k \in \mathbb{N}\right\}$ in algebra ' $a z+b^{\prime}$ ' into the algebraic basis $\left\{z^{n} \bullet t^{k} ; n \in \mathbb{Z}, k \in \mathbb{N}\right\}$ in algebra $\mathcal{R}_{q}\left(\mathbb{C}^{\times} \ltimes \mathbb{C}\right)$.

Thus, $\varphi$ is an isomorphism of algebras. Note then that $\varphi$ preserves the comultiplication on generators:

$$
(\varphi \otimes \varphi)(\varkappa(t))=(\varphi \otimes \varphi)(t \otimes 1+z \otimes t)=1 \boxminus t \otimes 1 \boxminus 1+z \boxminus 1 \otimes 1 \boxminus t=\varkappa(1 \boxminus t)=\varkappa(\varphi(t))
$$

and similarly,

$$
(\varphi \otimes \varphi)(\varkappa(z))=\varkappa(\varphi(z)), \quad(\varphi \otimes \varphi)\left(\varkappa\left(z^{-1}\right)\right)=\varkappa\left(\varphi\left(z^{-1}\right)\right)
$$

Since the comultiplication, like $\varphi$, is a homomorphism of algebras, this implies that the same formulas are true for the arguments of the form $z^{n} t^{k}$, and thus for all elements of algebra ' $a z+b$ '. We obtain that $\varphi$ preserves the comultiplication (on all elements). Similarly it is proved that $\varphi$ preserves counit and antipode (here we need to use the fact that the counit is a homomorphism, and antipode an antihomomorphism). Hence, $\varphi$ is an isomorphism of Hopf algebras.

\section{(d) Reflexivity of $\mathcal{R}_{q}\left(\mathbb{C}^{\times} \ltimes \mathbb{C}\right)$}

Reflexivity diagram for $\mathcal{R}_{q}\left(\mathbb{C}^{\times} \ltimes \mathbb{C}\right)$.

Theorem 7.8. For any $q \in \mathbb{C}^{\times}$the rigid Hopf algebra $\mathcal{R}_{q}\left(\mathbb{C}^{\times} \ltimes \mathbb{C}\right)$ is holomorphically reflexive, and

1) for $|q|=1$ its reflexivity diagram has the form

$$
\begin{aligned}
& \mathcal{R}_{q}\left(\mathbb{C}^{\times} \ltimes \mathbb{C}\right) \cong \mathcal{R}\left(\mathbb{C}^{\times}\right) \underset{\delta^{q}}{\stackrel{z}{\odot}} \mathcal{R}(\mathbb{C}) \quad \stackrel{\odot}{\longmapsto} \quad \mathcal{O}\left(\mathbb{C}^{\times}\right) \underset{\delta^{q}}{\stackrel{z}{\sim}} \mathcal{O}(\mathbb{C}) \cong \mathcal{O}_{q}\left(\mathbb{C}^{\times} \ltimes \mathbb{C}\right) \\
& { }^{\star 1} \quad I * \\
& \mathcal{R}_{q}^{\star}\left(\mathbb{C}^{\times} \ltimes \mathbb{C}\right) \cong \mathcal{R}^{\star}\left(\mathbb{C}^{\times}\right) \stackrel{\delta^{q}}{\odot} \mathcal{R}^{\star}(\mathbb{C}) \quad \stackrel{\odot}{\longleftarrow} \quad \mathcal{O}^{\star}\left(\mathbb{C}^{\times}\right) \underset{z}{\stackrel{\delta^{q}}{\odot}} \mathcal{O}^{\star}(\mathbb{C}) \cong \mathcal{O}_{q}^{\star}\left(\mathbb{C}^{\times} \ltimes \mathbb{C}\right)
\end{aligned}
$$

2) for $|q| \neq 1$ the form:

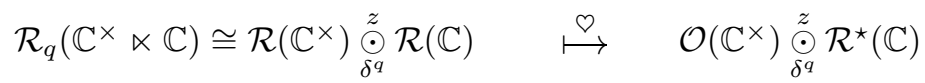

$$
\begin{aligned}
& \star 1 \quad I * \\
& \mathcal{R}_{q}^{\star}\left(\mathbb{C}^{\times} \ltimes \mathbb{C}\right) \cong \mathcal{R}^{\star}\left(\mathbb{C}^{\times}\right) \underset{z}{\stackrel{\delta^{q}}{\odot}} \mathcal{R}^{\star}(\mathbb{C}) \quad \stackrel{\odot}{\longleftarrow} \quad \mathcal{O}^{\star}\left(\mathbb{C}^{\times}\right) \underset{z}{\stackrel{\delta^{q}}{\odot}} \mathcal{R}(\mathbb{C})
\end{aligned}
$$


For symmetry one can note here that all the multipliers here $-\mathcal{R}(\mathbb{C}), \mathcal{R}^{\star}(\mathbb{C}), \mathcal{R}\left(\mathbb{C}^{\times}\right), \mathcal{R}^{\star}\left(\mathbb{C}^{\times}\right)$, $\mathcal{O}\left(\mathbb{C}^{\times}\right), \mathcal{O}^{\star}\left(\mathbb{C}^{\times}\right)$- are nuclear spaces and appear only in pair Fréchet-Fréchet and Brauner-Brauner. So if in diagrams (7.90)-(7.91) all (or some of) the injective tensor products $\odot$ are replaced by projective tensor products $\circledast$, then we shall obtain isomorphic diagrams.

The rest of this section is devoted to the proof of Theorem 7.8. We carry out it in three steps.

$\mathcal{R}_{q}\left(\mathbb{C}^{\times} \ltimes \mathbb{C}\right) \stackrel{\stackrel{P}{\rightarrow}}{\rightarrow} \mathcal{O}_{q}\left(\mathbb{C}^{\times} \ltimes \mathbb{C}\right)$ for $|q|=1$.

Proposition 7.11. For $|q|=1$ the algebra $\mathcal{O}_{q}\left(\mathbb{C}^{\times} \ltimes \mathbb{C}\right)$ is an Arens-Michael algebra, and the natural inclusion $\mathcal{R}_{q}\left(\mathbb{C}^{\times} \ltimes \mathbb{C}\right) \subseteq \mathcal{O}_{q}\left(\mathbb{C}^{\times} \ltimes \mathbb{C}\right)$ is an Arens-Michael envelope of the algebra $\mathcal{R}_{q}\left(\mathbb{C}^{\times} \ltimes \mathbb{C}\right)$ :

$$
\mathcal{R}_{q}\left(\mathbb{C}^{\times} \ltimes \mathbb{C}\right)^{\ominus}=\mathcal{O}_{q}\left(\mathbb{C}^{\times} \ltimes \mathbb{C}\right)
$$

Proof. This follows from the fact that for $|q|=1$ the seminorms (7.61) on $\mathcal{R}_{q}\left(\mathbb{C}^{\times} \ltimes \mathbb{C}\right)$ are submultiplicative.

$\mathcal{R}\left(\mathbb{C}^{\times}\right) \odot_{\delta^{q}}^{z} \mathcal{R}(\mathbb{C}) \stackrel{\odot}{\rightarrow} \mathcal{O}\left(\mathbb{C}^{\times}\right) \odot_{\delta^{q}}^{z} \mathcal{R}^{\star}(\mathbb{C})$ for $|q| \neq 1$. Recall that the structure of algebras $\mathcal{R}\left(\mathbb{C}^{\times}\right)$and $\mathcal{O}\left(\mathbb{C}^{\times}\right)$was discussed in $\S 3(\mathrm{c})$ In particular, we have noted there that the topology of $\mathcal{O}\left(\mathbb{C}^{\times}\right)$is generated by seminorms (3.47):

$$
\|u\|_{C}=\sum_{n \in \mathbb{Z}}\left|u_{n}\right| \cdot C^{|n|}, \quad C \geqslant 1 .
$$

In Example 5.2 noted also that these seminorms are submultiplicative. Let us state two more their properties: first, obviously,

$$
C \leqslant D \quad \Longrightarrow \quad\|u\|_{C} \leqslant\|u\|_{D}
$$

and, second, for $|q|<1$ we have

$$
|q|^{n} \leqslant|q|^{-|n|}, \quad n \in \mathbb{Z},
$$

This implies

$$
\left\|\mathrm{M}_{\delta^{q}}^{\star}(u)\right\|_{C}=\sum_{n \in \mathbb{Z}}\left|u_{n}\right| \cdot|q|^{n} \cdot C^{|n|} \leqslant \sum_{n \in \mathbb{Z}}\left|u_{n}\right| \cdot|q|^{-|n|} \cdot C^{|n|}=\sum_{n \in \mathbb{Z}}\left|u_{n}\right| \cdot\left(\frac{C}{|q|}\right)^{|n|}=\|u\|_{C /|q|}
$$

i.e.,

$$
\forall i \in \mathbb{N} \quad\left\|\left(\mathrm{M}_{\delta q}^{\star}\right)^{i}(u)\right\|_{C} \leqslant\|u\|_{C /|q|^{i}} \quad(|q|<1)
$$

Similarly, for $|q|>1$ we have

$$
|q|^{n} \leqslant|q|^{|n|}, \quad n \in \mathbb{Z}
$$

so

$$
\left\|\mathrm{M}_{\delta^{q}}^{\star}(u)\right\|_{C}=\sum_{n \in \mathbb{Z}}\left|u_{n}\right| \cdot|q|^{n} \cdot C^{|n|} \leqslant \sum_{n \in \mathbb{Z}}\left|u_{n}\right| \cdot|q|^{|n|} \cdot C^{|n|}=\sum_{n \in \mathbb{Z}}\left|u_{n}\right| \cdot(C \cdot|q|)^{|n|}=\|u\|_{C \cdot|q|}
$$

i.e.,

$$
\forall i \in \mathbb{N} \quad\left\|\left(\mathrm{M}_{\delta^{q}}^{\star}\right)^{i}(u)\right\|_{C} \leqslant\|u\|_{C \cdot|q|^{i}} \quad(|q|>1)
$$

In accordance with Proposition 7.10 let us identify in calculations the symbols $1 \square t$ and $z \square 1$ with the symbols $z$ and $t$ :

$$
1 \boxminus t \equiv t, \quad z \boxminus 1 \equiv z
$$

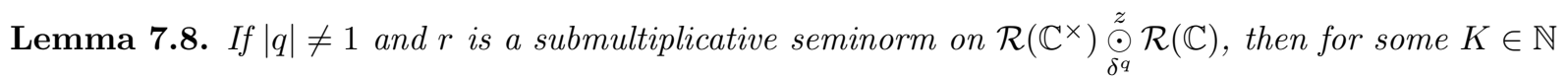

$$
\forall k>K \quad r\left(t^{k}\right)=0
$$


Proof. 1. Suppose first that $|q|<1$ and take $K \in \mathbb{N}$ such that $|q|^{K}<\frac{1}{r(z) \cdot r\left(z^{-1}\right)}$. Then for any $k>K$ we obtain $|q|^{k} \cdot r(z) \cdot r\left(z^{-1}\right)<1$, and so

$$
\begin{aligned}
r\left(t^{k}\right)=r\left(t^{k} \cdot z^{l} \cdot z^{-l}\right)=|q|^{l k} \cdot r\left(z^{l} \cdot t^{k} \cdot z^{-l}\right) \leqslant|q|^{l k} \cdot r\left(z^{l}\right) \cdot r\left(t^{k}\right) \cdot r\left(z^{-l}\right) \leqslant & \\
& \leqslant|q|^{l k} \cdot r(z)^{l} \cdot r(t)^{k} \cdot r\left(z^{-1}\right)^{l}=r(t)^{k} \cdot\left(|q|^{k} \cdot r(z) \cdot r\left(z^{-1}\right)\right)^{l} \underset{l \rightarrow \infty}{\longrightarrow} 0
\end{aligned}
$$

2. On the contrary, suppose $|q|>1$. Then we can take $K \in \mathbb{N}$ such that $|q|^{K}>r(z) \cdot r\left(z^{-1}\right)$, and for all $k>K$ we have $\frac{r(z) \cdot r\left(z^{-1}\right)}{|q|^{k}}<1$, so

$$
\begin{aligned}
& r\left(t^{k}\right)=r\left(t^{k} \cdot z^{-l} \cdot z^{l}\right)=|q|^{-l k} \cdot r\left(z^{-l} \cdot t^{k} \cdot z^{l}\right) \leqslant|q|^{-l k} \cdot r\left(z^{l}\right) \cdot r\left(t^{k}\right) \cdot r\left(z^{-l}\right) \leqslant \\
& \leqslant|q|^{-l k} \cdot r(z)^{l} \cdot r(t)^{k} \cdot r\left(z^{-1}\right)^{l}=r(t)^{k} \cdot\left(\frac{r(z) \cdot r\left(z^{-1}\right)}{|q|^{k}}\right)^{l} \underset{l \rightarrow \infty}{\longrightarrow} 0
\end{aligned}
$$

Proposition 7.12. 1) Suppose $|q|<1$, and $D \geqslant 1$ and $K \in \mathbb{N}$ are such that

$$
D \cdot|q|^{K} \geqslant 1
$$

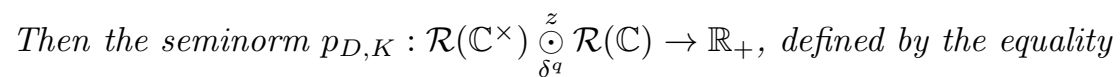

$$
p_{D, K}\left(\sum_{k \in \mathbb{N}} u_{k} \odot t^{k}\right)=\sum_{k=0}^{K} \underbrace{\left\|u_{k}\right\|_{D \cdot|q|^{k}}}_{\substack{\text { seminorm } \\ \text { [3.47 }}}=\sum_{k=0}^{K} \sum_{n \in \mathbb{Z}}\left|u_{k, n}\right| \cdot\left(D \cdot|q|^{k}\right)^{|n|}
$$

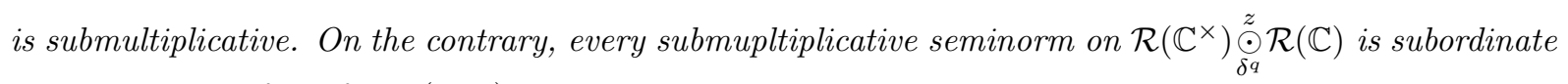
to a seminorm of the form (7.99).

2) If $|q|>1$, and $D \geqslant 1$ and $K \in \mathbb{N}$ are such that

$$
\frac{D}{|q|^{K}} \geqslant 1
$$

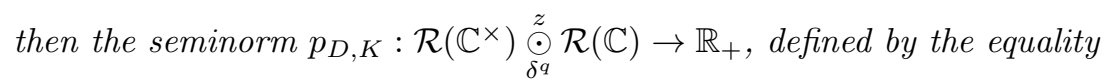

$$
p_{D, K}\left(\sum_{k \in \mathbb{N}} u_{k} \odot t^{k}\right)=\sum_{k=0}^{K} \underbrace{\left\|u_{k}\right\|_{\frac{D}{|q|^{K}}}}_{\substack{\text { seminorm } \\ \text { [3.47 }}}=\sum_{k=0}^{K} \sum_{n \in \mathbb{Z}}\left|u_{k, n}\right| \cdot\left(\frac{D}{|q|^{K}}\right)^{|n|}
$$

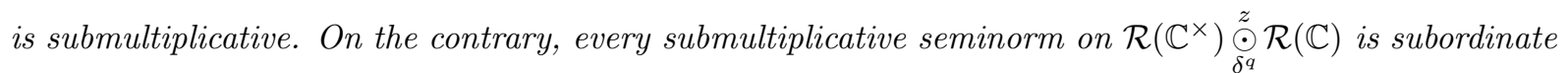
to some seminorm of the form (7.99).

Proof. Consider the case $|q|<1$. The submultiplicativity of the seminorm (7.99) is verified directly:

$$
\begin{aligned}
& p_{D, K}(u \cdot v)=\sum_{k=0}^{K}\left\|(u \cdot v)_{k}\right\|_{D \cdot|q|^{k}}=(\mathbf{7 . 1 9})=\sum_{k=0}^{K}\left\|\sum_{i=0}^{k} u_{i} \cdot\left(\mathrm{M}_{\delta^{q}}^{\star}\right)^{i}\left(v_{k-i}\right)\right\|_{D \cdot|q|^{k}}= \\
& =\sum_{k=0}^{K} \sum_{i=0}^{k}\left\|u_{i} \cdot\left(\mathrm{M}_{\delta^{q}}^{\star}\right)^{i}\left(v_{k-i}\right)\right\|_{D \cdot|q|^{k}} \leqslant \sum_{k=0}^{K} \sum_{i=0}^{k}\left\|u_{i}\right\|_{D \cdot|q|^{k}} \cdot\left\|\left(\mathrm{M}_{\delta^{q}}^{\star}\right)^{i}\left(v_{k-i}\right)\right\|_{D \cdot|q|^{k}} \leqslant(\underline{7.92)},(\underline{7.94}) \leqslant
\end{aligned}
$$




$$
\leqslant \sum_{k=0}^{K} \sum_{i=0}^{k}\left\|u_{i}\right\|_{D \cdot|q|^{i}} \cdot\left\|v_{k-i}\right\|_{D \cdot|q|^{k-i}} \leqslant\left(\sum_{i=0}^{K}\left\|u_{i}\right\|_{D \cdot|q|^{i}}\right) \cdot\left(\sum_{j=0}^{K}\left\|v_{j}\right\|_{D \cdot|q|^{j}}\right)=p_{D, K}(u) \cdot p_{D, K}(v)
$$

Then, let $r$ be a submultiplicative seminorm on $\mathcal{R}\left(\mathbb{C}^{\times}\right) \stackrel{z}{\odot} \mathcal{R}(\mathbb{C})$. Let us choose by Lemma 7.8 an integer $K \in \mathbb{N}$ such that (7.97) holds, and put

$$
L=\max _{0 \leqslant k \leqslant K} r\left(t^{k}\right)
$$

Note that $r$ is a submultiplicative seminorm on the subalgebra $1 \odot \mathcal{R}\left(\mathbb{C}^{\times}\right)$, consisting of functions of the form $a \odot 1, a \in \mathcal{R}\left(\mathbb{C}^{\times}\right)$, and isomorphic to $\mathcal{R}\left(\mathbb{C}^{\times}\right)$. Hence, on this subalgebra $r$, must be subordinate to some seminorm (3.47):

$$
r(a \odot 1) \leqslant M \cdot\|a\|_{C}, \quad a \in \mathcal{R}\left(\mathbb{C}^{\times}\right)
$$

for some $C \geqslant 1, M>0$. Choose now $D \geqslant 1$ such that

$$
C \leqslant D \cdot|q|^{K} \leqslant D \cdot|q|^{K-1} \leqslant \ldots \leqslant D \cdot|q| \leqslant D
$$

Then

$$
\begin{aligned}
r(u)=r\left(\sum_{k \in \mathbb{N}} u_{k} \odot t^{k}\right) \leqslant \sum_{k \in \mathbb{N}} r\left(u_{k}\right) \cdot r\left(t^{k}\right)=\sum_{k=0}^{K} r\left(u_{k}\right) \cdot r\left(t^{k}\right) \leqslant \sum_{k=0}^{K} M \cdot\left\|u_{k}\right\|_{C} \cdot L & = \\
& =L \cdot M \cdot \sum_{k=0}^{K}\left\|u_{k}\right\|_{D \cdot|q|^{k}}=L \cdot M \cdot p_{D, K}(u)
\end{aligned}
$$

Thus, $r$ is subordinate to some seminorm $p_{D, K}$.

The case $|q|>1$ is considered similarly, but instead of (7.94) we have to apply here formula (7.96), and instead of (7.103) the chain

$$
C \leqslant \frac{D}{|q|^{K}} \leqslant \frac{D}{|q|^{K-1}} \leqslant \ldots \leqslant \frac{D}{|q|} \leqslant D
$$

Proposition 7.13. For $|q| \neq 1$ the formula

$$
\zeta_{n} \odot t^{k} \mapsto z_{n} \odot \tau^{k}
$$

uniquely defines a homomorphism of Hopf algebras

$$
\mathcal{R}\left(\mathbb{C}^{\times}\right) \stackrel{\bigodot_{\delta^{q}}}{\mathcal{R}} \mathcal{R}(\mathbb{C}) \rightarrow \mathcal{O}\left(\mathbb{C}^{\times}\right) \underset{\delta^{q}}{\stackrel{z}{\odot}} \mathcal{R}^{\star}(\mathbb{C})
$$

which is an Arens-Michael envelope of the algebra $\mathcal{R}\left(\mathbb{C}^{\times}\right) \underset{\delta^{q}}{\stackrel{z}{\odot}} \mathcal{R}(\mathbb{C})$.

Proof. It remains to check here that $\mathcal{O}\left(\mathbb{C}^{\times}\right) \odot \mathcal{R}^{\star}(\mathbb{C})$ is a completion of $\mathcal{R}\left(\mathbb{C}^{\times}\right) \odot \mathcal{R}(\mathbb{C})$ with respect to the seminorms (7.99) or, depending on $q$, seminorms (7.101).

$\mathcal{O}^{\star}\left(\mathbb{C}^{\times}\right) \odot_{z}^{\delta^{q}} \mathcal{R}(\mathbb{C}) \stackrel{\ominus}{\rightarrow} \mathcal{R}^{\star}\left(\mathbb{C}^{\times}\right) \odot_{z}^{\delta^{q}} \mathcal{R}^{\star}(\mathbb{C})$ for arbitrary $q$. Consider the space $\mathcal{O}^{\star}\left(\mathbb{C}^{\times}\right)$. From the first formula in (7.85)

one can deduce the identity:

$$
\mathrm{M}_{z}^{\star}\left(\zeta_{n}\right)=\zeta_{n-1},
$$

$$
\left(\mathrm{M}_{z}^{\star}\right)^{k}(\alpha)=\left(\mathrm{M}_{z}^{\star}\right)^{k}\left(\sum_{n \in \mathbb{Z}} \alpha_{n} \cdot \zeta_{n}\right)=\sum_{n \in \mathbb{Z}} \alpha_{n} \cdot \zeta_{n-k}=\sum_{m \in \mathbb{Z}} \alpha_{m+k} \cdot \zeta_{m}
$$


Recall seminorms $\|\cdot\|_{N}$ on $\mathcal{O}^{\star}\left(\mathbb{C}^{\times}\right)$, defined by formulas (6.12):

$$
\|\alpha\|_{N}=\sum_{|n| \leqslant N}\left|\alpha_{n}\right|, \quad N \in \mathbb{N}, \quad \alpha=\sum_{n \in \mathbb{Z}} \alpha_{n} \cdot \zeta_{n}
$$

Note the following their properties:

$$
M \leqslant N \quad \Longrightarrow \quad\|\beta\|_{M} \leqslant\|\beta\|_{N}
$$

and

$$
\left.\left\|\left(\mathrm{M}_{z}^{\star}\right)^{i}(\beta)\right\|_{M}=\sum_{|n| \leqslant M}\left|\left(\mathrm{M}_{z}^{\star}\right)^{i}(\beta)_{n}\right|=\mid \underline{7.105}\right)=\sum_{|n| \leqslant M}\left|\beta_{n+i}\right| \leqslant \sum_{|n| \leqslant M+i}\left|\beta_{n}\right|=\|\beta\|_{M+i}
$$

By Theorem (17.6), the general formula for multiplication in the algebra $\mathcal{O}^{\star}\left(\mathbb{C}^{\times}\right) \underset{z}{\odot^{q}} \mathcal{R}(\mathbb{C})$ has the form

$$
\alpha \cdot \beta=\sum_{k \in \mathbb{N}}\left(\sum_{i=0}^{k} \alpha_{i} *\left(\mathbf{M}_{z}^{\star}\right)^{i}\left(\beta_{k-i}\right)\right) \odot t^{k}
$$

and on basis elements looks as follows:

$$
\begin{aligned}
\zeta_{m} \odot t^{k} \cdot \zeta_{n} \odot t^{l} & =(\mathbf{7 . 1 4})=\zeta_{m} *\left(\mathrm{M}_{z}^{\star}\right)^{k}\left(\zeta_{n}\right) \odot t^{k+l}=(7.85) \\
& =\zeta_{m} * \zeta_{n-k} \odot t^{k+l}=\left\{\begin{array}{ll}
\zeta_{m} \odot t^{k+l}, & m=n-k \\
0, & m \neq n-k
\end{array}= \begin{cases}\zeta_{m} \odot t^{k+l}, & m+k=n \\
0, & m+k \neq n\end{cases} \right.
\end{aligned}
$$

Lemma 7.9. Every continuous submultiplicative seminorm $p$ on the algebra $\mathcal{O}^{\star}\left(\mathbb{C}^{\times}\right) \stackrel{\delta^{q}}{\odot} \mathcal{R}(\mathbb{C})$ vanishes on almost all elements of the basis $\left\{\zeta_{n} \odot t^{k} ; n \in \mathbb{Z}, k \in \mathbb{N}\right\}$ (i.e. on all but a finite subfamily):

$$
\operatorname{card}\left\{(n, k) \in \mathbb{Z} \times \mathbb{N}: p\left(\zeta_{n} \odot t^{k}\right) \neq 0\right\}<\infty
$$

Proof. Put $p_{n, k}=p\left(\zeta_{n} \odot t^{k}\right)$ and note that

$$
p_{m, k+l} \leqslant p_{m, k} \cdot p_{m+k, l}, \quad k, m \in \mathbb{N}, n \in \mathbb{Z}
$$

Indeed, from (7.109) it follows that

$$
\zeta_{m} \odot t^{k+l}=\zeta_{m} \odot t^{k} \cdot \zeta_{m+k} \odot t^{l}
$$

so

$$
p_{m, k+l}=p\left(\zeta_{m} \odot t^{k+l}\right) \leqslant p\left(\zeta_{m} \odot t^{k}\right) \cdot p\left(\zeta_{m+k} \odot t^{l}\right)=p_{m, k} \cdot p_{m+k, l}
$$

Consider the sets

$$
S_{k}=\left\{n \in \mathbb{Z}: p_{n, k} \neq 0\right\}
$$

and note the following two things.

1. From the continuity and submupltiplicativity of a seminorm $p$ on $\mathcal{R}(\mathbb{C}) \stackrel{\delta^{q}}{\odot_{z}} \mathcal{O}^{\star}(\mathbb{C} \times)$ it follows that the functional

$$
p_{0}(\alpha)=p(\alpha \odot 1)=p\left(\alpha \odot t^{0}\right), \quad \alpha \in \mathcal{O}^{\star}\left(\mathbb{C}^{\times}\right)
$$

is a continuous submultiplicative seminorm on $\mathcal{O}^{\star}\left(\mathbb{C}^{\times}\right)$:

$$
p_{0}(\alpha * \beta)=p((\alpha * \beta) \odot 1)=p((\alpha \odot 1) \cdot(\beta \odot 1)) \leqslant p(\alpha \odot 1) \cdot p(\beta \odot 1)=p_{0}(\alpha) \cdot p_{0}(\beta)
$$


This means, by Lemma 6.2 that $p_{0}$ is subordinate to some seminorm of the form (6.12):

$$
p_{0}(\alpha) \leqslant C \cdot\|\alpha\|_{N}=C \cdot \sum_{|n| \leqslant N}\left|\alpha_{n}\right|, \quad \alpha \in \mathcal{O}^{\star}\left(\mathbb{C}^{\times}\right)
$$

This in its turn implies that the set $S_{0}=\left\{n \in \mathbb{Z}: p_{0}\left(\zeta_{n}\right)=p\left(1 \odot \zeta_{n}\right) \neq 0\right\}$ must be finite:

$$
\operatorname{card} S_{0}<\infty
$$

2. From the inequalities (7.110) one can deduce the following implications:

$$
\left\{\begin{array} { l } 
{ p _ { m , k + 1 } \leqslant p _ { m , k } \cdot p _ { m + k , 1 } } \\
{ p _ { m , k + 1 } \leqslant p _ { m , k + 1 } \cdot p _ { m + k + 1 , 0 } }
\end{array} \Longrightarrow \left\{\begin{array}{l}
S_{k+1} \subseteq S_{k} \\
S_{k+1} \subseteq S_{0}-(k+1)
\end{array} \quad \Longrightarrow \quad S_{k+1} \subseteq S_{k} \cap\left(S_{0}-k-1\right)\right.\right.
$$

(here $S-i$ means the shift of the set $S$ by $i$ units to left on the group $\mathbb{Z}$ ). So we can conclude that $S_{k}$ form a tapering chain of finite (since $S_{0}$ is finite) sets:

$$
S_{0} \supseteq S_{1} \supseteq \ldots \supseteq S_{k} \supseteq S_{k+1} \supseteq \ldots
$$

And at the minimum after the number $K=\max S_{0}-\min S_{0}$ this chain vanishes:

$$
S_{0} \supseteq S_{1} \supseteq \ldots \supseteq S_{K} \supseteq S_{K+1}=\varnothing
$$

(since $\left.S_{K+1} \subseteq S_{0} \cap\left(S_{0}-K-1\right)=\varnothing\right)$.

Proposition 7.14. For any $N \in \mathbb{N}$ the functional

$$
r_{N}(\alpha)=r_{N}\left(\sum_{k \in \mathbb{N}} \alpha_{k} \odot t^{k}\right)=\sum_{k=0}^{N}\left\|\alpha_{k}\right\|_{N-k}=\sum_{k=0}^{N} \sum_{|n| \leqslant N-k}\left|\alpha_{n, k}\right|
$$

is a continuous submultiplicative seminorm on $\mathcal{R}(\mathbb{C}) \underset{z}{\delta^{q}} \mathcal{O}^{\star}\left(\mathbb{C}^{\times}\right)$. Every continuous submultiplicative seminorm on $\mathcal{R}(\mathbb{C}) \underset{z}{\delta^{q}} \mathcal{O}^{\star}\left(\mathbb{C}^{\times}\right)$is subordinated to some seminorm $r_{N}$.

Proof. The functional $r_{N}$ is a continuous seminorm because $\alpha \mapsto \alpha_{k}$ are linear continuous mappings:

$$
r_{N}(\lambda \cdot \alpha+\beta)=\sum_{k=0}^{N}\left\|\lambda \cdot \alpha_{k}+\beta_{k}\right\|_{N-k} \leqslant|\lambda| \cdot \sum_{k=0}^{N}\left\|\alpha_{k}\right\|_{N-k}+\sum_{k=0}^{N}\left\|\beta_{k}\right\|_{N-k}=|\lambda| \cdot r_{N}(\alpha)+r_{N}(\beta)
$$

Let us consider the submultiplicativity:

$$
\begin{aligned}
& r_{N}(\alpha \cdot \beta)=(17.108)=r_{N}\left(\sum_{k \in \mathbb{N}} \sum_{i=0}^{k} \alpha_{i} *\left(\mathrm{M}_{z}^{\star}\right)^{i}\left(\beta_{k-i}\right) \odot t^{k}\right)=\sum_{k=0}^{N}\left\|\sum_{i=0}^{k} \alpha_{i} *\left(\mathrm{M}_{z}^{\star}\right)^{i}\left(\beta_{k-i}\right)\right\|_{N-k} \leqslant \\
& \leqslant \sum_{k=0}^{N} \sum_{i=0}^{k}\left\|\alpha_{i}\right\|_{N-k} \cdot\left\|\left(\mathrm{M}_{z}^{\star}\right)^{i}\left(\beta_{k-i}\right)\right\|_{N-k} \leqslant \underline{7.107)} \leqslant \sum_{k=0}^{N} \sum_{i=0}^{k}\left\|\alpha_{i}\right\|_{N-k} \cdot\left\|\beta_{k-i}\right\|_{N-k+i} \leqslant \\
& \leqslant \sum_{k=0}^{N} \sum_{i=0}^{k} \underbrace{\left\|\alpha_{i}\right\|_{N-k}} \cdot\left\|\beta_{k-i}\right\|_{N-(k-i)} \leqslant \sum_{k=0}^{N} \sum_{i=0}^{k}\left\|\alpha_{i}\right\|_{N-i} \cdot\left\|\beta_{k-i}\right\|_{N-(k-i)} \leqslant \\
& \| \begin{array}{c}
\left\|\alpha_{i}\right\|_{N-i}, \\
\text { since } \\
k \geqslant i
\end{array} \\
& k \geqslant i \text {, } \\
& \text { hence } \\
& N-k \leqslant N-i, \\
& \text { and this allows }
\end{aligned}
$$




$$
\leqslant\left(\sum_{i=0}^{N}\left\|\alpha_{i}\right\|_{N-i}\right) \cdot\left(\sum_{j=0}^{N}\left\|\beta_{j}\right\|_{N-j}\right)=r_{N}(\alpha) \cdot r_{N}(\beta)
$$

It remains to check that every continuous submultiplicative seminorm $p$ on $\mathcal{R}(\mathbb{C}) \underset{z}{\delta^{q}} \mathcal{O}^{\star}\left(\mathbb{C}^{\times}\right)$is subordinate to some seminorm $r_{N}$. This follows from Lemma [7.9; since $p$ vanishes on almost all basis elements $t^{k} \odot \zeta_{n}$, we can find a number $N \in \mathbb{N}$ such that

$$
\left\{(n, k) \in \mathbb{Z} \times \mathbb{N}: p\left(\zeta_{n} \odot t^{k}\right) \neq 0\right\} \subseteq\{(n, k) \in \mathbb{Z} \times \mathbb{N}: k \leqslant N \&|n| \leqslant N-k\}
$$

Then we put

$$
C=\max \left\{p\left(\zeta_{n} \odot t^{k}\right) ;(n, k): k \leqslant N,|n| \leqslant N-k\right\}
$$

and obtain:

$$
\begin{array}{r}
p(\alpha)=p\left(\sum_{n \in \mathbb{Z}, k \in \mathbb{N}} \alpha_{n, k} \cdot \zeta_{n} \odot t^{k}\right) \leqslant \sum_{n \in \mathbb{Z}, k \in \mathbb{N}}\left|\alpha_{n, k}\right| \cdot p\left(\zeta_{n} \odot t^{k}\right)=\sum_{k=0}^{N} \sum_{|n| \leqslant N-k}\left|\alpha_{n, k}\right| \cdot p\left(\zeta_{n} \odot t^{k}\right) \leqslant \\
\leqslant C \cdot \sum_{k=0}^{N} \sum_{|n| \leqslant N-k}\left|\alpha_{n, k}\right|=(7.111)=C \cdot r_{N}(\alpha)
\end{array}
$$

Proposition 7.15. The formula

$$
\zeta_{n} \odot t^{k} \mapsto \zeta_{n} \odot \tau^{k}
$$

defines a homomorphism of Hopf algebras

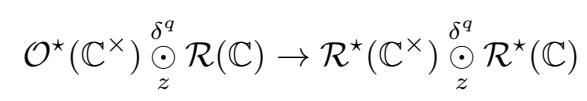

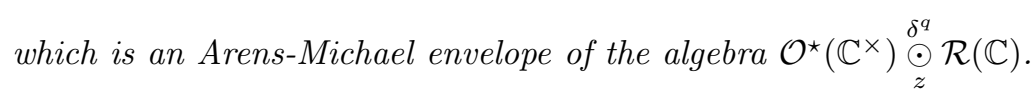

Proof. It remains only to check that $\mathcal{R}^{\star}\left(\mathbb{C}^{\times}\right) \odot \mathcal{R}^{\star}(\mathbb{C})$ is a completion of $\mathcal{O}^{\star}\left(\mathbb{C}^{\times}\right) \odot \mathcal{R}(\mathbb{C})$ with respect to seminorms (7.111). 


\section{Bibliography}

[1] S. S. Akbarov. "Pontryagin duality in the theory of topological vector spaces and in topological algebra." Journal of Mathematical Sciences. 113(2):179-349 (2003).

[2] S. S. Akbarov. "Stereotype spaces, algebras, homologies: An outline," In: Topological Homology (A. Ya. Helemskii, Ed.), Nova Science Publishers (2000), pp. 1-29.

[3] S. S. Akbarov. "Pontryagin duality and topological algebras", In: "Topological Algebras, their Applications and Related Topics", eds. Krzysztof Jarosz and Andrzej Soltysiak, Banach Center Publications 67: $55-71,2005$.

[4] V. A. Artamonov, V. N. Salii, L. A. Skornyakov, L. N. Shevrin, E. G. Shul'geifer. Obshchaya algebra. Tom 2. (Russian) [General algebra. Vol. 2] Edited and with a preface by Skornyakov. Spravochnaya Matematicheskaya Biblioteka. [Mathematical Reference Library] "Nauka", Moscow, 1991. 480 pp. ISBN: 5-02-014427-4

[5] N. Bourbaki. Espaces vectoriels topologiques. Chaps. I-V, Hermann, Paris.

[6] N. Bourbaki. Topologie Générale. Chaps. III-VIII, Hermann, Paris.

[7] K. Brauner. "Duals of Frechet spaces and a generalization of the Banach-Dieudonne theorem." Duke Math. Jour. 40(4):845-855, 1973.

[8] V. Chari, A. Pressley. A guide to quantum groups. Cambridge university press, 1995.

[9] S. Dăscălescu, C. Năstăsescu, Ş. Raianu. Hopf algebras, Marcel Dekker, 2001.

[10] M. Enock, J.-M. Schwartz. Kac Algebras and Duality of Locally Compact Groups. Springer-Verlag, 1992.

[11] M. Enock, J.-M. Schwartz. "Une dualité dans les algèbres de von Neumann." Note C. R. Acad. Sc. Paris 277 (1973), 683-685.

[12] M. Enock, J.-M. Schwartz. "Une catégorie d'algèbres de Kac." Note C. R. Acad. Sc. Paris 279 (1974), 643-645.

[13] M. Enock, J.-M. Schwartz. "Une dualité dans les algèbres de von Neumann." Supp. Bull. Soc. Math. France Mémoire 44 (1975), 1-144.

[14] H. Grauert, R. Remmert. Theory of Stein spaces. Springer, 1977.

[15] R. A. Horn, C. R. Johnson. Martix analysis. Cambridge university press.

[16] E. Hewitt, K. A. Ross. Abstract Harmonic Analysis, Vol. 2, Springer (1963).

[17] H. Jarchow. Locally convex spaces. B.G.Teubner, Stuttgart, 1981.

[18] S. Kakutani, V. Klee. "The finite topology of a linear space," Arch. Math. 14(1):55-58, 1963. 
[19] C. Kassel. Quantum groups. Springer.

[20] M. Krein. A principle of duality for bicompact groups and quadratic block algebras. (Russian) Doklady Akad. Nauk SSSR (N.S.) 69:725-728, 1949.

[21] J. Kustermans, S. Vaes. "Locally compact quantum groups," Ann. scient. Èc. Norm. Sup. 4è série, $33: 837-934,2000$.

[22] B. Ya. Levin. Lectures on entire functions. AMS, 1996.

[23] S. MacLane. Categories for the working mathematician. Springer, Berlin, 1971.

[24] K.-H. Neeb. Holomorphy and Convexity in Lie Theory. Walter de Gruyter, 2000.

[25] A. Pietsch. Nukleare Lokalkonvexe Raume, Akademie-Verlag, Berlin (1965).

[26] A. Yu. Pirkovskii. Arens-Michael envelopes, homological epimorphisms and relatively quasifree algebras. (Russian) Trudy MMO, 69: 34-123, 2008.

[27] L. Pontrjagin. "The theory of topological commutative groups," Ann. Math. 35(2): 361-388, 1934.

[28] J. Kustermans, W. Pusz, P. M. Sołtan, S. Vaes, A. Van Daele, L. Vainerman, S. L. Woronowicz. "Locally compact quantum groups," In: "Quantum symmetry in noncommutative geometry" (P. M. Hajak, Ed.), Locally compact quantum groups. Lecture Notes School / Conference on Noncommutative Geometry and Quantum groups, Warsaw, 2001, Banach Center Publications, to appear.

[29] N. Saavedra Rivano. "Catègories Tannakiennes," Lecture Notes in Mathematics, no. 265, Springer, 1972.

[30] W. Rudin. Functional analysis. McGraw-Hill, 1973.

[31] M. F. Smith. "The Pontrjagin duality theorem in linear spaces." Annals of Mathematics, 1952, $56(2): 248-253$.

[32] H. H. Shaeffer. Topological Vector Spaces. Macmillan (1966).

[33] B. V. Shabat. Introduction to complex analysis, Vol. I, Nauka, Moscow, 1985.

[34] B. V. Shabat. Introduction to complex analysis, Vol. II, Nauka, Moscow, 1985.

[35] P. Schauenburg. "On the Braiding on a Hopf Algebra in a Braided Category", New York J. Math. 4: 259-263 (1998).

[36] R. Street. Quantum Groups: a path to current algebra, Series: Australian Mathematical Society Lecture Series (No. 19), Cambridge, 2007.

[37] M. E. Sweedler. "Cocommutative Hopf algebras with antipode," Bull. Amer. Math. Soc., 73(1):126$128,1967$.

[38] J. L. Taylor. Several complex variables with connections to algebraic geometry and Lie groups. Graduate Studies in Mathematics, V. 46. - AMS, Providence, Rhode Island, 2002.

[39] A. Van Daele. "Dual pairs of Hopf *-algebras", Bull. Lond. Math. Soc., 25:209-230 (1993).

[40] A. Van Daele, "The Haar measure on some locally compact quantum groups," http://arxiv.org/abs/math/0109004v1

[41] A. Van Daele. "Multiplier Hopf algebras", Trans. Amer. Math. Soc., 342: 917-932, 1994. 
[42] A. Van Daele. "An algebraic framework for group duality", Adv. Math. 140:323-366, 1998.

[43] L. I. Vainerman. A characterization of objects that are dual to locally compact groups. (Russian) Funkcional. Anal. i Prilozen. 8(1):75-76, 1974.

[44] L. I. Vainerman, G. I. Kac. Nonunimodular ring groups, and Hopf-von Neumann algebras. (Russian) Dokl. Akad. Nauk SSSR. 211: 1031-1034, 1973.

[45] L. I. Vainerman, G. I. Kac. Nonunimodular ring groups and Hopf-von Neumann algebras. (Russian) Mat. Sb. (N.S.) 94(136):194-225, 1974.

[46] E. B. Vinberg, A. L. Onishchik. Seminar po gruppam Li i algebraicheskim gruppam. (Russian) [A seminar on Lie groups and algebraic groups] Second edition. URSS, Moscow, 1995. 344 pp. ISBN: $5-88417-083-1$

[47] S. Wang. "Quantum 'ax + b' group as quantum automorphism group of $k[x]$," http://arxiv.org/abs/math/9807094v2.

[48] S. L. Woronowicz. "Quantum 'az + b' group on complex plane." Int. J. Math. 12(4):461-503, 2001.

[49] S. Zhang, "Braided Hopf algebras," arXiv:math.RA/0511251 v8 25 May 2006. 


\section{Contents}

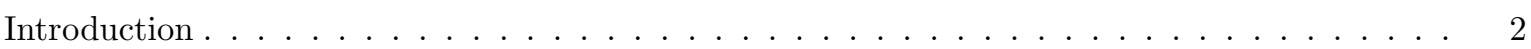

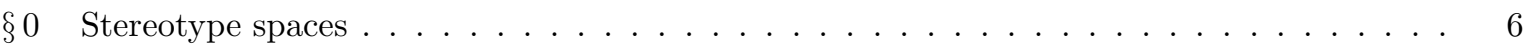

(a) Definition and typical examples . . . . . . . . . . . . . . . . 7

(b) Smith space generated by a compact set . . . . . . . . . . . . . 8

(c) Brauner spaces generated by an expanding sequence of compact sets . . . . . . . . 10

(d) Projective Banach systems and injective Smith systems . . . . . . . . . . . . . 10

(e) Banach representation of a Smith space . . . . . . . . . . . . . . . . 12

(f) Injective systems of Banach spaces, generated by compact sets . . . . . . . . . 13

(g) Nuclear stereotype spaces . . . . . . . . . . . . . . . . . . . . . 16

(h) Spaces $\mathbb{C}^{M}$ and $\mathbb{C}_{M} \ldots \ldots \ldots \ldots \ldots \ldots \ldots$

Space of functions $\mathbb{C}^{M} \ldots \ldots \ldots \ldots \ldots$

The space of point charges $\mathbb{C}_{M} \ldots \ldots \ldots \ldots \ldots$. . . . . . . . . . . . . . . . . . . . .

Duality between $\mathbb{C}^{M}$ and $\mathbb{C}_{M} \ldots \ldots \ldots \ldots$. . . . . . . . . . . . . . . . . . . .

Bases in $\mathbb{C}^{M}$ and $\mathbb{C}_{M} \ldots \ldots \ldots \ldots \ldots$

$\S 1$ Stereotype Hopf algebras . . . . . . . . . . . . . . . . . . . . . 21

(a) Tensor products and the structure of monoidal category on $\mathfrak{S t e}_{\mathfrak{1}} \ldots \ldots \ldots$. . . . . 21

(b) Stereotype Hopf algebras . . . . . . . . . . . . . . . . . 23

Algebras, coalgebras and Hopf algebras in a symmetric monoidal category. . 23

Projective and injective stereotype algebras. . . . . . . . . . . . . . 25

Stereotype Hopf algebras. . . . . . . . . . . . . . . . . . . . . . . . . . . . . . . . .

Duality for stereotype Hopf algebras. . . . . . . . . . . . . . . . 26

Dual pairs. . . . . . . . . . . . . . . . . . . . 27

(c) Key example: Hopf algebras $\mathbb{C}^{G}$ and $\mathbb{C}_{G} \ldots \ldots \ldots \ldots \ldots$. . . . . . . . . . . . . . . . . . . . . . .

Algebra $\mathbb{C}^{G}$ of functions on $G$. . . . . . . . . . . . . . . . 27

The algebra $\mathbb{C}_{G}$ of point charges on $G \ldots \ldots \ldots \ldots \ldots$

$\mathbb{C}^{G}$ and $\mathbb{C}_{G}$ as stereotype Hopf algebras. . . . . . . . . . . . . . . . 28

(d) Sweedler's notations and the stereotype approximation property . . . . . . . . 34

(e) Grouplike elements . . . . . . . . . . . . . . . . . . . . 36

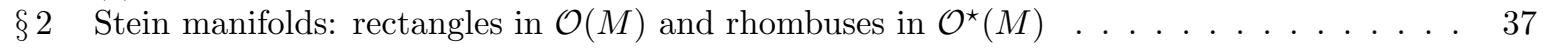

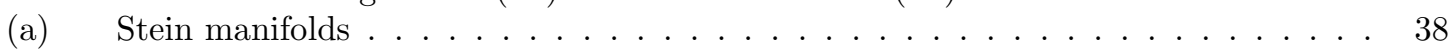

(b) Outer envelopes on $M$ and rectangles in $\mathcal{O}(M) \ldots \ldots \ldots$

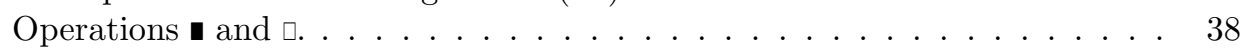

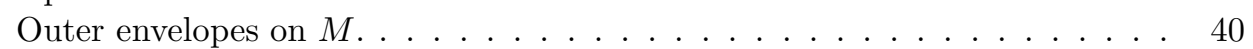

Rectangles in $\mathcal{O}(M) \ldots \ldots \ldots \ldots \ldots \ldots$. . . . . . . . . . . . . . . . . . . . . . . . . . . . . . .

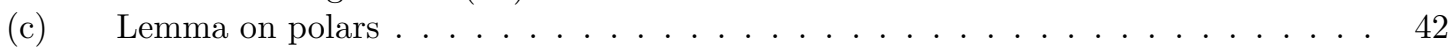

(d) Inner envelopes on $M$ and rhombuses in $\mathcal{O}^{\star}(M) \ldots \ldots \ldots \ldots$

Operations and $\diamond \ldots \ldots \ldots \ldots \ldots \ldots$

Inner envelopes on $M \ldots \ldots \ldots \ldots \ldots \ldots$. . . . . . . . . . . . . . . . . . . . . . . . . . .

Rhombuses in $\mathcal{O}^{\star}(M) \ldots \ldots \ldots \ldots \ldots$

(e) Duality between rectangles and rhombuses . . . . . . . . . . . . . . . . . 46

$\S 3$ Stein groups and Hopf algebras connected to them . . . . . . . . . . . . . . . . . . 48 
(a) Stein groups, linear groups and algebraic groups . . . . . . . . . . . . . . . . . . 48

(b) Hopf algebras $\mathcal{O}(G), \mathcal{O}^{\star}(G), \mathcal{R}(G), \mathcal{R}^{\star}(G) \ldots \ldots \ldots \ldots \ldots$

Hopf algebras $\mathcal{O}(G)$ and $\mathcal{O}^{\star}(G)$ on a Stein group $G$. . . . . . . . . . . . . 50

Hopf algebras $\mathcal{R}(G)$ and $\mathcal{R}^{\star}(G)$ on an affine algebraic group $G$. . . . . . . . 51

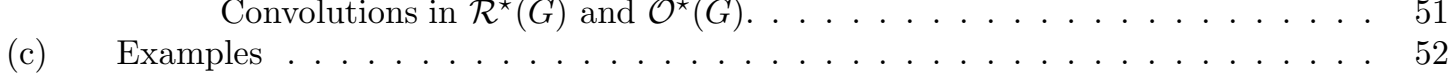

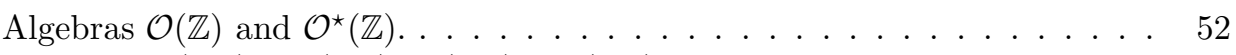

Algebras $\mathcal{R}\left(\mathbb{C}^{\times}\right), \mathcal{R}^{\star}\left(\mathbb{C}^{\times}\right), \mathcal{O}\left(\mathbb{C}^{\times}\right), \mathcal{O}^{\star}\left(\mathbb{C}^{\times}\right) \ldots \ldots \ldots$

The chain $\mathcal{R}(\mathbb{C}) \subset \mathcal{O}(\mathbb{C}) \subset \mathcal{O}^{\star}(\mathbb{C}) \subset \mathcal{R}^{\star}(\mathbb{C}) \ldots \ldots \ldots$. . . . . . . . . . . 57

$\S 4$ Functions of exponential type on a Stein group . . . . . . . . . . . . . . . 63

(a) Semicharacters and inverse semicharacters on Stein groups . . . . . . . . . . 63

(b) Submultiplicative rhombuses and dually submultiplicative rectangles . . . . . . . . 66

(c) Holomorphic functions of exponential type . . . . . . . . . . . . . . . . 69

Algebra $\mathcal{O}_{\text {exp }}(G)$ of holomorphic functions of exponential type. . . . . . . . 69

Algebra $\mathcal{O}_{\exp }^{\star}(G)$ of exponential analytic functionals. . . . . . . . . . . 71

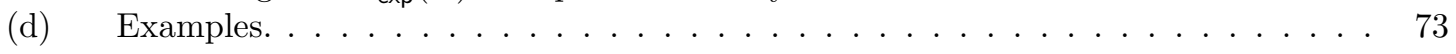

Finite groups. . . . . . . . . . . . . . . . . . . . . . . . . . . . . .

Groups $\mathbb{C}^{n} \ldots \ldots \ldots \ldots \ldots \ldots \ldots \ldots \ldots$. . . . . . . . . . . . . . . . . . . . . . . . . . . .

Groups $\mathrm{GL}_{n}(\mathbb{C}) \ldots \ldots \ldots \ldots \ldots \ldots \ldots$

(e) Injection $b_{G}: \mathcal{O}_{\exp }(G) \rightarrow \mathcal{O}(G) \ldots \ldots \ldots \ldots \ldots$

(f) Nuclearity of the spaces $\mathcal{O}_{\exp }(G)$ and $\mathcal{O}_{\exp }^{\star}(G) \ldots \ldots \ldots \ldots \ldots$

(g) Holomorphic mappings of exponential type and tensor products of the spaces $\mathcal{O}_{\exp }(G)$ and $\mathcal{O}_{\text {exp }}^{\star}(G) \ldots \ldots \ldots \ldots \ldots \ldots \ldots$

(h) Structure of Hopf algebras on $\mathcal{O}_{\exp }(G)$ and $\mathcal{O}_{\text {exp }}^{\star}(G) \ldots \ldots \ldots$

$\S 5$ Arens-Michael envelope and holomorphic reflexivity . . . . . . . . . . . . . . 86

(a) Submultiplicative seminorms and Arens-Michael algebras . . . . . . . . . . . . . . 86

(b) Arens-Michael envelopes ................................ 88

(c) The mapping $b_{G}^{\star}: \mathcal{O}^{\star}(G) \rightarrow \mathcal{O}_{\text {exp }}^{\star}(G)$ is an Arens-Michael envelope . . . . . . . . 89

(d) The mapping $b_{G}: \mathcal{O}_{\exp }(G) \rightarrow \mathcal{O}(G)$ is an Arens-Michael envelope for the groups with algebraic connected component of identity . . . . . . . . . . . . . . . . . . .

(e) Holomorphic reflexivity . . . . . . . . . . . . . . . . . . . . . 94

$\S 6$ Holomorphic reflexivity as a generalization of Pontryagin duality . . . . . . . . . . . . 96

(a) Pontryagin duality for compactly generated Stein groups . . . . . . . . . . . . . . . . . . . . . . . . . . . . .

(b) Fourier transform as an Arens-Michael envelope . . . . . . . . . . . . . . . . . . 96

Finite Abelian group. . . . . . . . . . . . . . . . . . . . . . . . . . . . . . . . . . . . . . . . . . .

Complex plane $\mathbb{C} \ldots \ldots \ldots \ldots \ldots$. . . . . . . . . . . . . . . . . . . . . . . . . . . . . . . .

Complex circle $\mathbb{C}^{\times} \ldots \ldots \ldots \ldots \ldots$

Group of integers $\mathbb{Z}$. . . . . . . . . . . . . . . . . . . . . . . . . . . . . . .

Proof of Theorem 6.2 . . . . . . . . . . . . . . . . . . . . . . . . . . . . . . . . . .

(c) Inclusion diagram ............................. 105

$\S 7$ Appendix: holomorphic reflexivity of the quantum group ' $a z+b$ ' . . . . . . . . . 106

(a) Quantum combinatorial formulas . . . . . . . . . . . . . . . 106

(b) Hopf algebra of skew polynomials and similar constructions . . . . . . . . . . . 107

Tensor products $X \odot \mathcal{R}(\mathbb{C}), X \circledast \mathcal{R}(\mathbb{C}), X \odot \mathcal{R}^{\star}(\mathbb{C}), X \circledast \mathcal{R}^{\star}(\mathbb{C})$. . . . . . 107

Algebras of skew polynomials $A \stackrel{\varphi}{\odot} \mathcal{R}(\mathbb{C})$ and skew power series $A \stackrel{\varphi}{\circledast} \mathcal{R} \star(\mathbb{C}) \quad 108$

Quantum pairs in stereotype Hopf algebras. . . . . . . . . . . . . . . 108

Hopf algebras $H \odot_{\omega}^{z} \mathcal{R}(\mathbb{C})$ and $H^{\star} \circledast_{z}^{\omega} \mathcal{R}^{\star}(\mathbb{C})$. . . . . . . . . . . . . . . . . 109

Chains $H \odot_{\omega}^{z} \mathcal{R}(\mathbb{C}) \subset H \odot_{\omega}^{z} \mathcal{O}(\mathbb{C}) \subset H \odot_{\omega}^{z} \mathcal{O}^{\star}(\mathbb{C}) \subset H \odot_{\omega}^{z} \mathcal{R}^{\star}(\mathbb{C})$ and $H \circledast_{\omega}^{z} \mathcal{R}(\mathbb{C}) \subset H \circledast \circledast_{\omega}^{z} \mathcal{O}(\mathbb{C}) \subset H \circledast_{\omega}^{z} \mathcal{O}^{\star}(\mathbb{C}) \subset H \circledast_{\omega}^{z} \mathcal{R}^{\star}(\mathbb{C}) . \quad . \quad 115$

(c) Quantum group ' $a z+b^{\prime}=\mathcal{R}_{q}\left(\mathbb{C}^{\times} \ltimes \mathbb{C}\right) \ldots \ldots \ldots \ldots \ldots \ldots \ldots$

The group $\mathbb{C}^{\times} \ltimes \mathbb{C}$ of affine transformation of a complex plane. . . . . . . . 117

Stereotype algebras $\mathcal{R}\left(\mathbb{C}^{\times} \ltimes \mathbb{C}\right)$ and $\mathcal{R}^{\star}\left(\mathbb{C}^{\times} \ltimes \mathbb{C}\right) \ldots \ldots . . . \ldots 117$ 
Stereotype algebras $\mathcal{O}\left(\mathbb{C}^{\times} \ltimes \mathbb{C}\right)$ and $\mathcal{O}^{\star}\left(\mathbb{C}^{\times} \ltimes \mathbb{C}\right) \ldots \ldots \ldots . \ldots . \ldots 120$ $\mathcal{R}\left(\mathbb{C}^{\times} \ltimes \mathbb{C}\right), \mathcal{R}^{\star}\left(\mathbb{C}^{\times} \ltimes \mathbb{C}\right), \mathcal{O}\left(\mathbb{C}^{\times} \ltimes \mathbb{C}\right)$ and $\mathcal{O}^{\star}\left(\mathbb{C}^{\times} \ltimes \mathbb{C}\right)$ as Hopf algebras. . 120 Hopf algebras $\mathcal{R}_{q}\left(\mathbb{C}^{\times} \ltimes \mathbb{C}\right), \mathcal{R}_{q}^{\star}\left(\mathbb{C}^{\times} \ltimes \mathbb{C}\right), \mathcal{O}_{q}\left(\mathbb{C}^{\times} \ltimes \mathbb{C}\right), \mathcal{O}_{q}^{\star}\left(\mathbb{C}^{\times} \ltimes \mathbb{C}\right) . . . .122$ $\mathcal{R}_{q}\left(\mathbb{C}^{\times} \ltimes \mathbb{C}\right)$ as an algebra with generators and defining relations. . . . . . . 124

(d) Reflexivity of $\mathcal{R}_{q}\left(\mathbb{C}^{\times} \ltimes \mathbb{C}\right) \ldots \ldots \ldots \ldots \ldots \ldots \ldots \ldots$

Reflexivity diagram for $\mathcal{R}_{q}\left(\mathbb{C}^{\times} \ltimes \mathbb{C}\right)$. . . . . . . . . . . . 125 $\mathcal{R}_{q}\left(\mathbb{C}^{\times} \ltimes \mathbb{C}\right) \stackrel{\rho}{\rightarrow} \mathcal{O}_{q}\left(\mathbb{C}^{\times} \ltimes \mathbb{C}\right)$ for $|q|=1 \ldots \ldots \ldots . \ldots \ldots$

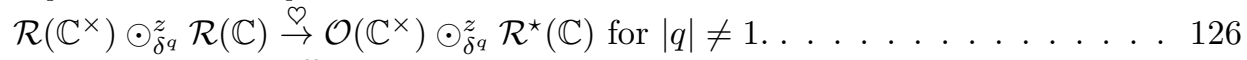
$\mathcal{O}^{\star}\left(\mathbb{C}^{\times}\right) \odot_{z}^{\delta^{q}} \mathcal{R}(\mathbb{C}) \stackrel{\ominus}{\rightarrow} \mathcal{R}^{\star}\left(\mathbb{C}^{\times}\right) \odot_{z}^{\delta^{q}} \mathcal{R}^{\star}(\mathbb{C})$ for arbitrary $q \ldots \ldots . . . . . .128$

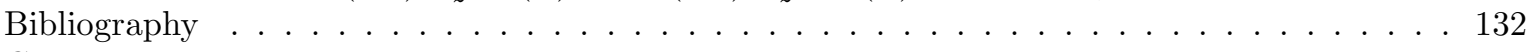

Contents . . . . . . . . . . . . . . . . . . . . . . 135 